
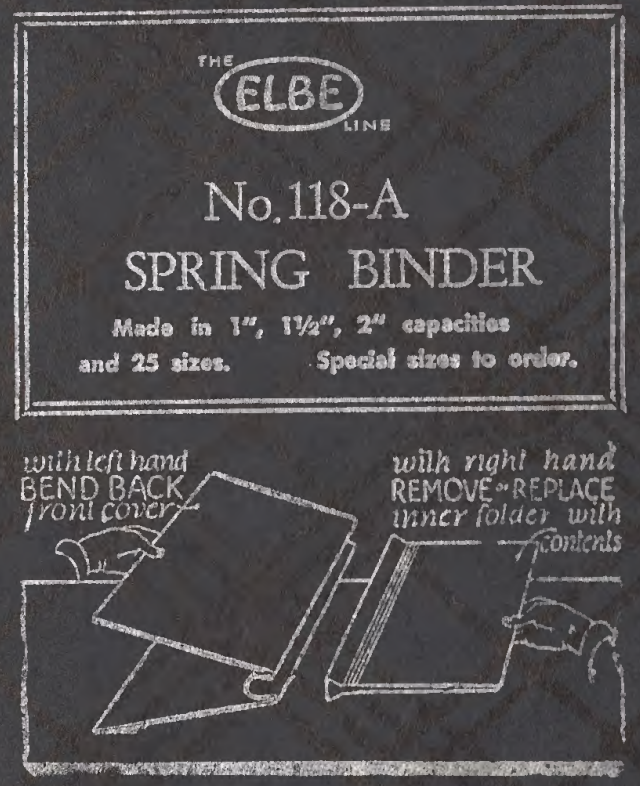

Made in U. S. A. 



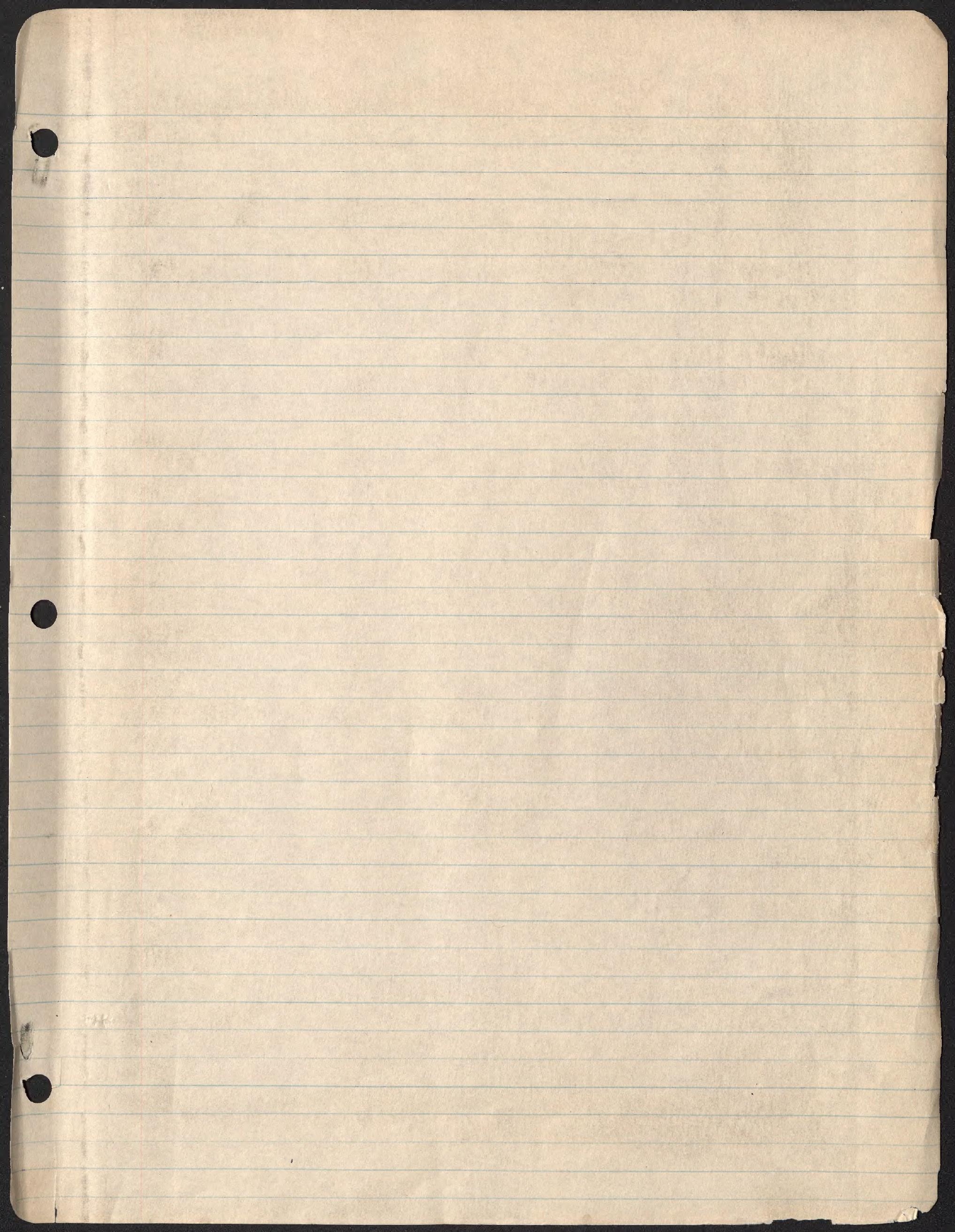





\section{Field Catalog}

\section{9}

Sixth ArChBOHD Expedition to New Guinea

Hobart Merritt Van Deusen

S IEPARTED: NeW YORK MarCh 13

L ARRIVED: NEWGUINEA MARCh 22

$\{$ IJeparted: New Guinea Dec. 13

A ARrIVED: Australia DEC.13

$\{$ DEPARTED: AUSTRALIF FEB.13,1960

A ARRIVED: NEW YORT FEB. 21

WITH SOME ADDITIONS FROM

AUSTRALIA BY PERSONAL

COLLECTING AND BYGIFT.

SeE: Results of the ArChBold Expeditions. No. 86 L.T. Brass SumMARY of thE SIXTH ARCHBOLD EXPEDITION TO NEW GUINEA (1959) Bull. Amer. Mus. Nat. Hist. Vol.127, Art. 4, 1964.

[EXPEDITION GEAR DID NOT ARRIYE IN LAF UNTIL APRIL 20, 1959.]

(NO INDIA INT UNTIL 4.25.59!) 
Q

0

0

orit 
(1) 6 TH ArChBOLd EXPEDition to New GuINEA $(1959)$

\begin{tabular}{|c|c|c|c|c|c|}
\hline 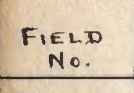 & NAmE & SEx & $\begin{array}{l}1959 \\
\text { JPATE }\end{array}$ & LOCALTY & ELEVATION \\
\hline 5001 & PERAMELES 160345 & $z_{j}$ & DIED MRR, 12 & N. QUEENSLAND: INNISFA & QI,M,R: 535 \\
\hline $\begin{array}{l}2 \\
3\end{array}$ & WALLABY ${ }_{192266^{\circ}}$ & ? & & 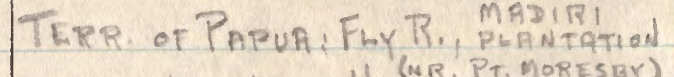 & \\
\hline $\begin{array}{l}3 \\
4\end{array}$ & WHALE 190864 & & & 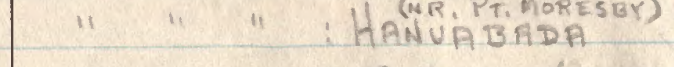 & ON REEF \\
\hline $\begin{array}{r}4 \\
05\end{array}$ & SMINTHOPSIS & of & $M A R, 23$ & " " "inr. Pr. Moresby (Tiaba & PLANTATIOAN \\
\hline & SMINTHOP 19124. & 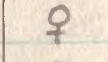 & MAR.23 & " " ": " n " L" & \\
\hline & PHALANGER O: & f & 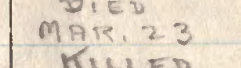 & "1. "BRUBAUGUIAR PLA & NTATION \\
\hline 8 & $\begin{array}{l}\text { PHALANGER } 1900^{\circ} \\
\text { PHOEV }\end{array}$ & 8 & $M A R \cdot 24$ & "4 " & $"$ \\
\hline 9 & PHALANGER MP" & 8 & $"$ & " " " : & \\
\hline 010 & PHALAANGER M: & o & "1. & TERP OE NGG": " & $10 \mathrm{~m}$. \\
\hline 11 & & 8 & {$\left[\begin{array}{c}A \cup G,-5 E P T_{1} \\
1958\end{array}\right]$} & $\begin{array}{c}\text { HERR OF N.G. : LAE } \\
\text { "I }\end{array}$ & . \\
\hline 12. & Srconyct 191237 s & q & Mar, 28 & " & $25 \mathrm{~m}$. \\
\hline 13 & 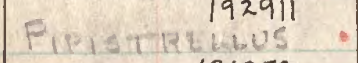 & 6 & MAR. 29 & " $(5$ mi.N.W. & $50 \mathrm{~m}$. \\
\hline $\begin{array}{l}14 \\
15\end{array}$ & "11 $19 / 358$ & ठ & 11 & " " & $50 \mathrm{~m}$. \\
\hline 16 & RATTUS $19315265^{\circ}$ & $q$ & "1 & " $1 "$ & $25 \mathrm{mi}$ \\
\hline $\begin{array}{l}16 \\
17\end{array}$ & ST GTPE Possum" & ? & MAR.29) & " : ? & $=$ \\
\hline $\begin{array}{l}17 \\
18\end{array}$ & NYETIMESE & $\delta$ & MAR. 30 & : LAE & $40 \mathrm{~m}$. \\
\hline $\begin{array}{l}18 \\
19\end{array}$ & 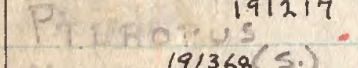 & बे & "1 & 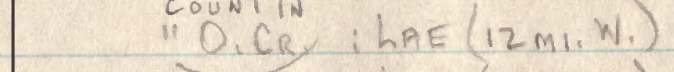 & $40 \mathrm{~m}$ \\
\hline $\begin{array}{l}19 \\
20\end{array}$ & Ming p p $192957^{3}$. & 0 & " & " $\quad$ i haE $(8 \mathrm{MI}, W)$ & $40 \mathrm{~m}$ \\
\hline & 192958 & & "1" & "1 & "11 \\
\hline & $19 / 359$ & & 11 & " & " \\
\hline $\begin{array}{l}22 \\
23\end{array}$ & $\begin{array}{l}\text { PIPISTRELLUS } \\
192166\end{array}$ & $f$ & Apr. 3 & T.N,G, : LAE (5 MI, WEST) & $50 \mathrm{~m}$ \\
\hline 24 & BANDIC00T/192167 & $=$ & " & " & $50-100 \mathrm{~m}$ \\
\hline 502.5 & 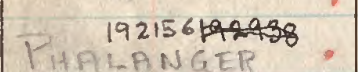 & ? & $"$ & " & " \\
\hline 26 & MiniopTERUS 1929. & $\hat{\sigma}$ & APR. 5 & 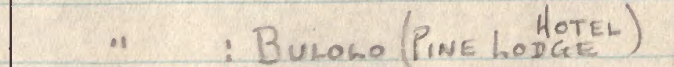 & $680 \mathrm{~m}$. \\
\hline 27 & PIPISTRELLUS. & q & APR. 7 & : LAE & $50 m$ \\
\hline 28 & PTEROPUS $19 / 2 / 3$. & $\sigma$ & " & $:$ & " \\
\hline 29 & HIPPOSIDER & & " 8 & :ZENAG & \\
\hline 301 & & & " & " & \\
\hline
\end{tabular}

* 1500 Presented to VAN D. Mar. 20 by Dr. (Mrs.) M. T. Mackirkas QLD. InST. OF MED. RES., HERSTON RD. HERSTON N 9 , BRI MISS RUTH SMITH, ASST, TO DR. MACKIRRAS Dr. DOROTHY F. SANDARS, ENDOPARASITES (MET IN 1948) \#15010,15011 GIFT: TOHDE, GUNN. TALL GRASS IN HOUSE YARD, EDGE OF FIR STRIP; $1 / 2$ MILE FROM SEA.

\#15013,15014 Shot at Tohn Kewly's - nerr MarkHam Road - near Rain fortest 


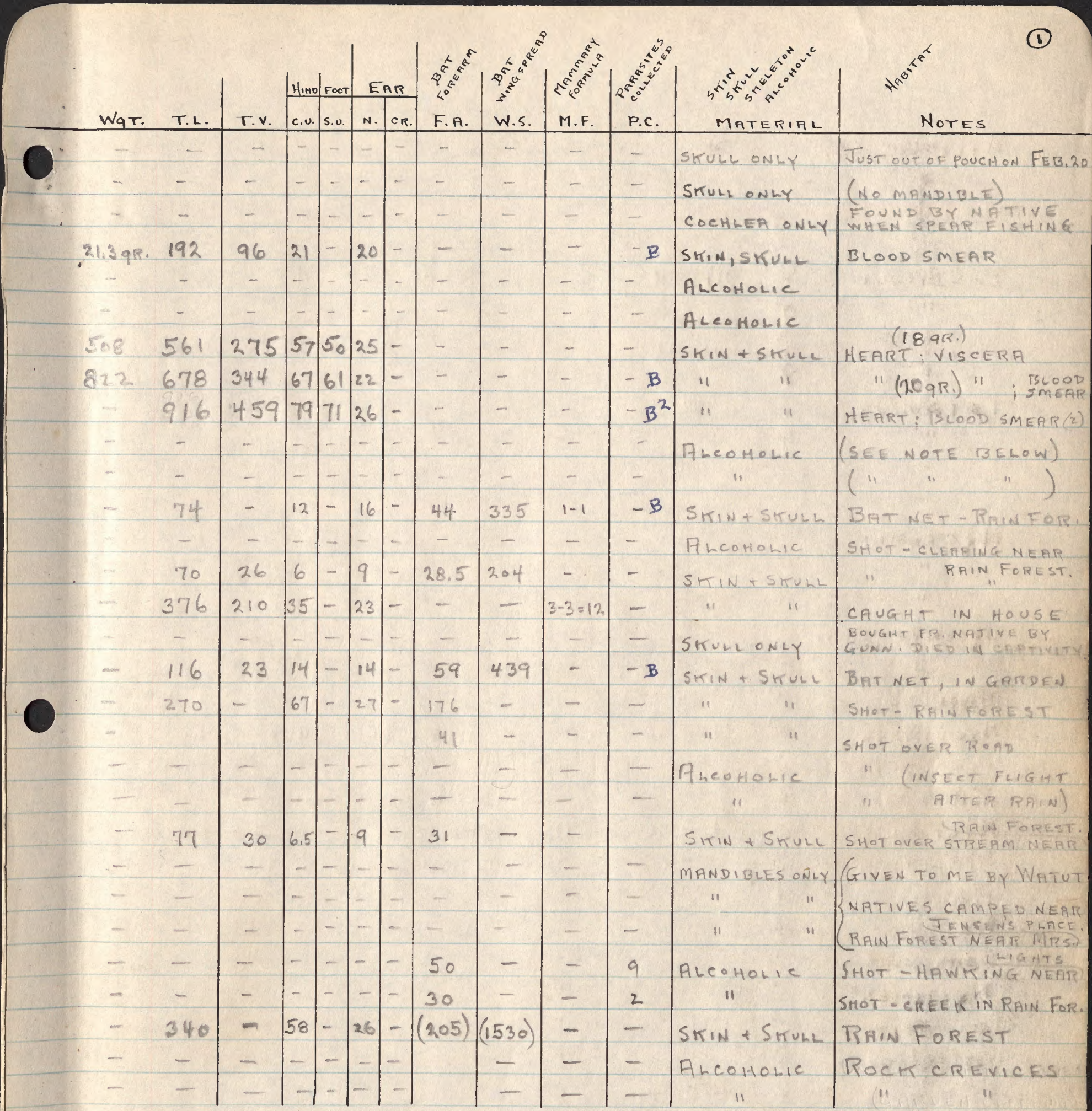

\#15002-15009 GIFT: KEN SLATER, FORMERLY OF PORT MORESBY ALL OF THIS MATERIAL WAS ALIVE IN CAGES AT HILA, 3 MILES ERST OF PORT MOTEESBY.

\# 15002 (LETTER 5 FEB 13.1959 TO h. J.B.) - "3 FEet HIGH, GREYISH TO GINGER"

\# 15016 GIFT: TIE. GUN N. DIED IN CAPTIVITY. BURIED. DUG UREY VAN DEUSEN. \# 15029 - 15033 - GIFT: RICHARD LEAFY - BOUGHT FROM NATIVES WHO FOUND THEM IN ROCKS IN REMNANT FOREST ON HILLS ABOVEZENAG 
-

1 



\begin{tabular}{|c|c|c|c|c|c|}
\hline $\begin{array}{l}\text { FIELD } \\
\text { NO. }\end{array}$ & NAME & SEX & DATE & LOCALITY & ELEVATION \\
\hline 15031 & HIPPOSIDEROS. & & APR. 8 & TERR, N.G, I ZENAG & 4000 ? \\
\hline 32 & 11192857 & & " & $" 1$ & \\
\hline 33 & $11192858^{\circ}$ & & 11 & $"$ & \\
\hline 34 & PETAURUS & $\delta$ & "1 & $\because \quad: Z E N A G(2 M i, N . W)$. & OVER $3000^{\prime}$; \\
\hline 15035 & BANDICOOT. & $q$ & APR. 9 & : LAE (3MIN.) & c. $15 \mathrm{M}$ \\
\hline 36 & $11 \quad 193124$ & का & 4 & 11 & \\
\hline 37 & $11 \quad 193125$ & q & " & 11 & \\
\hline 38 & $11 \frac{193126}{19}$ & $q$ & 11 & 11 & \\
\hline 39 & PETAURU' 191048 & $q$ & $" 1$ & 11 & c. $15 \mathrm{~m}$ \\
\hline 15040 & 11191049 & $q$ & 11 & 4 & \\
\hline 41 & $11 \quad 193110=$ & P.YG & 11 & 11 & \\
\hline 42 & $11 \quad 193111$ & P. YG & 11 & 11 & \\
\hline 43 & NYETIMENE. & $\delta$ & 11 & $\begin{array}{l}\text { "BOTANIE } \\
\text { GARDEN }\end{array}$ & $40 \mathrm{~m}$. \\
\hline 44 & $1 \quad 11191288$. & $\delta$ & 11 & $n$ & 11 \\
\hline 15045 & $11 \quad 191289$ & $\delta$ & "1 & $"$ & is \\
\hline 46 & SYCONYCTERIS, & $\delta$ & "1 & "u & 11 \\
\hline 47 & if 191236 . & $\hat{6}$ & 11 & "11 & 11 \\
\hline 48 & $\begin{array}{c}191232 \\
\text { MACROGLOSSUS. } \\
192253\end{array}$ & 8 & 11 & " & $20 \mathrm{M}$. \\
\hline 49 & Nyet IME 192756 & d & APR. 10 & $\because$ : LAE (BOT. GARD.) & $20 \mathrm{M}$ \\
\hline 15050 & $\begin{array}{l}\text { SYConYCTEPIS: } \\
190859\end{array}$ & q & 18 & " & $20 m$ \\
\hline 51 & $\begin{array}{c}\text { ZAGLOS5US } \\
191291\end{array}$ & 9 & 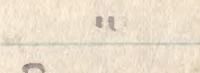 & ": SEPIK DIST; TELEFOMIN & \\
\hline 52 & NYCTIMEN/ & 우 & Apr. $\|$ & T. N,G, M.SCHULTZ PLANTATION & c. $100 \mathrm{~m}$. \\
\hline 53 & MELOMY's . & q & 11 & MOROBE DISTRICT & 1. \\
\hline 54 & RATTUS EX. & q & 11 & OOMsis (22 ROAD & 4 \\
\hline 15055 & RATT US R. & ? & it & mi. W.s.w. of LAE) & 11 \\
\hline 56 & EEHYMIPERA & 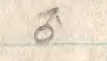 & 4 & " & it \\
\hline 57 & NYCTIMENE & 8 & " & $"$ & is \\
\hline 58 & $\begin{array}{l}191175 \\
\text { Pitalanger miteVLATUS }\end{array}$ & $\delta$ & APR. 12 & 11 (NEAREY) & $?$ \\
\hline 59 & RATTUS EXULANS - & $q$ & 11 & 11 & c. $100 \mathrm{M}$. \\
\hline 150601 & $11791535=1$ & 우 & APR. 13 & $" 1$ & 11 \\
\hline
\end{tabular}

ZENAG IS THE FARM + RANCH OF MICHAEL (MICK) TAMES LEAHY 52 ROAD MILES FROM LAE (ON ROAD T. BULOLO + WAU). ON DESOLATE TUNAI GRASS - COVERED HILLS. REHE FOREST ON SLOPES ABOVE HOUSE + IN GULLIES. ALTITUDE IN DISPUTE. (L.T.B. $-3600^{\prime}$ RICHARDLEAHY ( 17 YRS.OLD) $4400^{\prime}$ )

\#15034 - Wampit Piver Valley - Flows N. into the Markham R. ELEV. 3400 FT. (PER TICHARD LEAHY) PERAKLES VILLAGE LAND. 
(2)

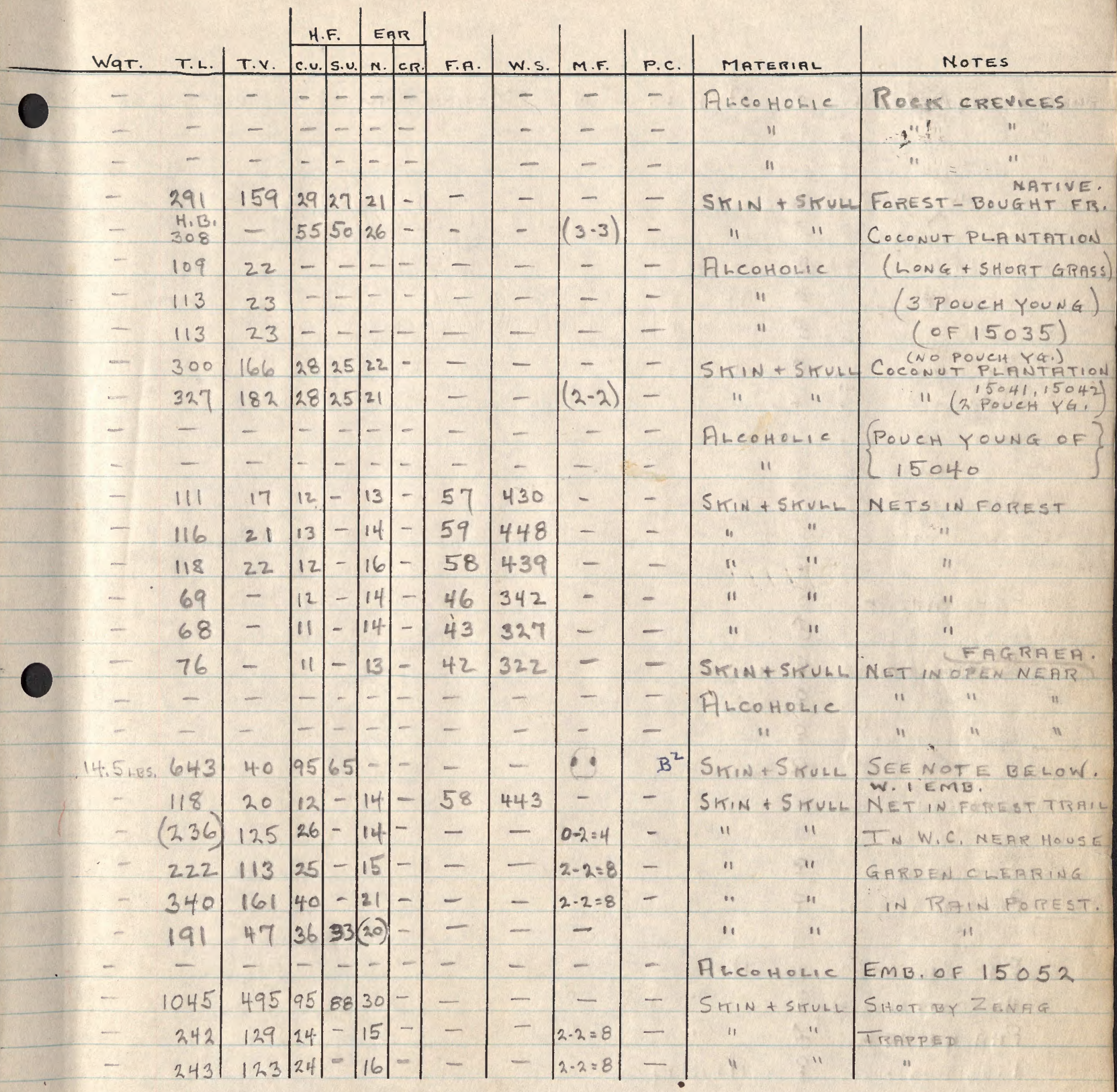

\# 15048 CAUGHT IN NET SET IN PLANTING OF FAGRAEA FRAGRANS FIR OM MaLaY PENINSULA (Malay "TEMBUSU"). PLANTED ON OPEN LAWN.

\# 15051 SENT to haE VIA WEWAK from Telefomin by Dept. of Agric. TO JOHN WORMESLEY, DEPT. OF FORESTS. DEAD ON ARRIVAL. GIFT T. ARCH. EXP. FROM J. WORMESLEY.

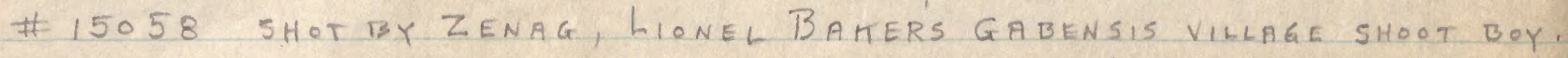
IN RAIN FOREST 1 OR 2 MILES FROM OOMSIS ( NE.) 




\begin{tabular}{|c|c|c|c|c|c|c|c|}
\hline $\begin{aligned} \text { FIELD } \\
\text { NO. }\end{aligned}$ & NAmE & SEX & $\begin{array}{l}1959 \\
\text { DATE } \\
\end{array}$ & \multicolumn{3}{|c|}{ LOCALITY } & Elevation \\
\hline 15061 & ECHYMIPERA & $\pi$ & APR. 13 & T.N.C & $B E$ & Msis & c. $100 \mathrm{M}$ \\
\hline 62 & TAPHOZOUS? & d & Apre. 14 & $"$ & 11 & 11 & c. $100 \mathrm{~m}$. \\
\hline 63 & $\begin{array}{l}191190 \\
\text { PHALANGER OR? }\end{array}$ & 운 & 11 & " & $"$ & " & 11 \\
\hline 64 & 1119119111 & 우 & 11 & $" 1$ & 11 & "1 & "1 \\
\hline 15065 & MELOMYS 191866 & $\delta$ & 11 & 11 & 11 & 11 & $\mathrm{c} .120 \mathrm{~m}$. \\
\hline 66 & 11191868 & ㅇ․ & 11 & " & 11 & 4 & 11 \\
\hline 67 & PIPISTRELLUS. & 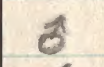 & APR. 15 & "1 & $"$ & $\because$ & c. $100 \mathrm{M}$. \\
\hline 68 & 11191361. & $\pi$ & $\because$ & " & $"$ & $"$ & . \\
\hline 69 & $\begin{array}{c}191238 \\
\text { SyCoNyCTERIS }\end{array}$ & $\hat{\sigma}$ & APR. 16 & "1 & 11 & is & $"$ \\
\hline 15070 & $\begin{array}{l}\text { EURYOT } 15 \\
\text { RHINOLORHUS } 19132^{3} \text {. }\end{array}$ & $?$ & 11 & "1 & 4 & $" 1$ & $\because$ \\
\hline 71 & $\begin{array}{r}191363 \\
\text { PipISTREI16,5 }\end{array}$ & $q$ & 11 & $" 1$ & "1 & 11 & 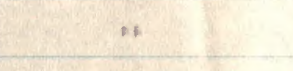 \\
\hline 72 & ECHYMIPERA & q. & 4 & " & " & "11 & $" 1$ \\
\hline 73 & MELOMYS & $\delta$ & "I & 1" & "11 & 11 & "11 \\
\hline 74 & $\begin{array}{r}191176 \\
\text { PHALANGTR }\end{array}$ & 2 & a1 & "I & "1 & 11 & $2-300=$ \\
\hline 15075 & $\begin{array}{r}191178 \\
\end{array}$ & $q$ & 4 & "1 & 11 & 11 & " ? \\
\hline 76 & $\begin{array}{c}190970 \\
\text { ECHYMIPRR }\end{array}$ & $\delta$ & $\because$ & 11 & 11 & $\prime \prime$ & c. $100 \mathrm{M}$ \\
\hline 77 & Pipistretifus. & $\delta$ & Apr. 17 & 11 & II & 4 & "i \\
\hline 78 & $\begin{array}{c}192840 \\
\text { EMBALE }\end{array}$ & q & 11 & 11 & 11 & 4 & 11 \\
\hline 79 & 11191305 & $q$ & 11 & 11 & "1 & 4 & i1 \\
\hline 15080 & $\begin{array}{l}192848(5) \\
\text { Hipposip } 5 \pi 05 \text {. }\end{array}$ & q & II & "1 & 4 & 11 & 11 \\
\hline 81 & 11 19/324(s). & q & 11 & 11 & 11 & ii & 4 \\
\hline 82 & $\begin{array}{c}191214 \\
\text { PAEROPUS N }\end{array}$ & $\pi$ & 11 & "1 & $" 1$ & " & 31 \\
\hline 83 & $11 \quad 191215$ & $q$ & 11 & "1 & "1 & 11 & 11 \\
\hline 84 & $\begin{array}{c}191219 \\
\text { DobSON 19 }\end{array}$ & ㅇ & 11 & 11 & "1 & $n$ & 11 \\
\hline 15085 & PHALAHGER M: & q & "1 & 11 & $" 1$ & 4 & $?$ \\
\hline 86 & 11 $\quad \frac{193134 .}{191620}$ & 47 & $" 1$ & $" 1$ & c. & " & ? \\
\hline 87 & RATTUS RUB: & 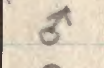 & APR. 18 & is: & “ & $n$ & $\mathrm{C.} .100 \mathrm{~m}$. \\
\hline 88 & HIPPOSIDEROS (s) & $q$ & $"$ & $\because$ & 4 & " & 11 \\
\hline 89 & PHALANGER M. & $\delta$ & $" 1$ & "1 & $"$ & " & $200 \mathrm{~m} ?$ \\
\hline 15090 & PTEROPUS NEO. & q & APR. 19 & 11 & "I & i1 & $130 \mathrm{~m}$ ? \\
\hline
\end{tabular}

\#15051 (CONT) "CULMEN" - TIP SAONT TOHAIR WINE (MID-DORSAL) - 115 CIR. SNOUT (AT BASE) - TO

TIP SNOUT TO ANT. CORNER OF EYE - 135

EYE SWT - 10

POUCH" ARER $130 \times 60$. MILK AREAS $1 / 3$ DIST. FROM ANT OF POUS \#15062 3 II FOLDS DORSALLY; 3 JU FOLDS VENT:; 4 III FOLDS VENT. 



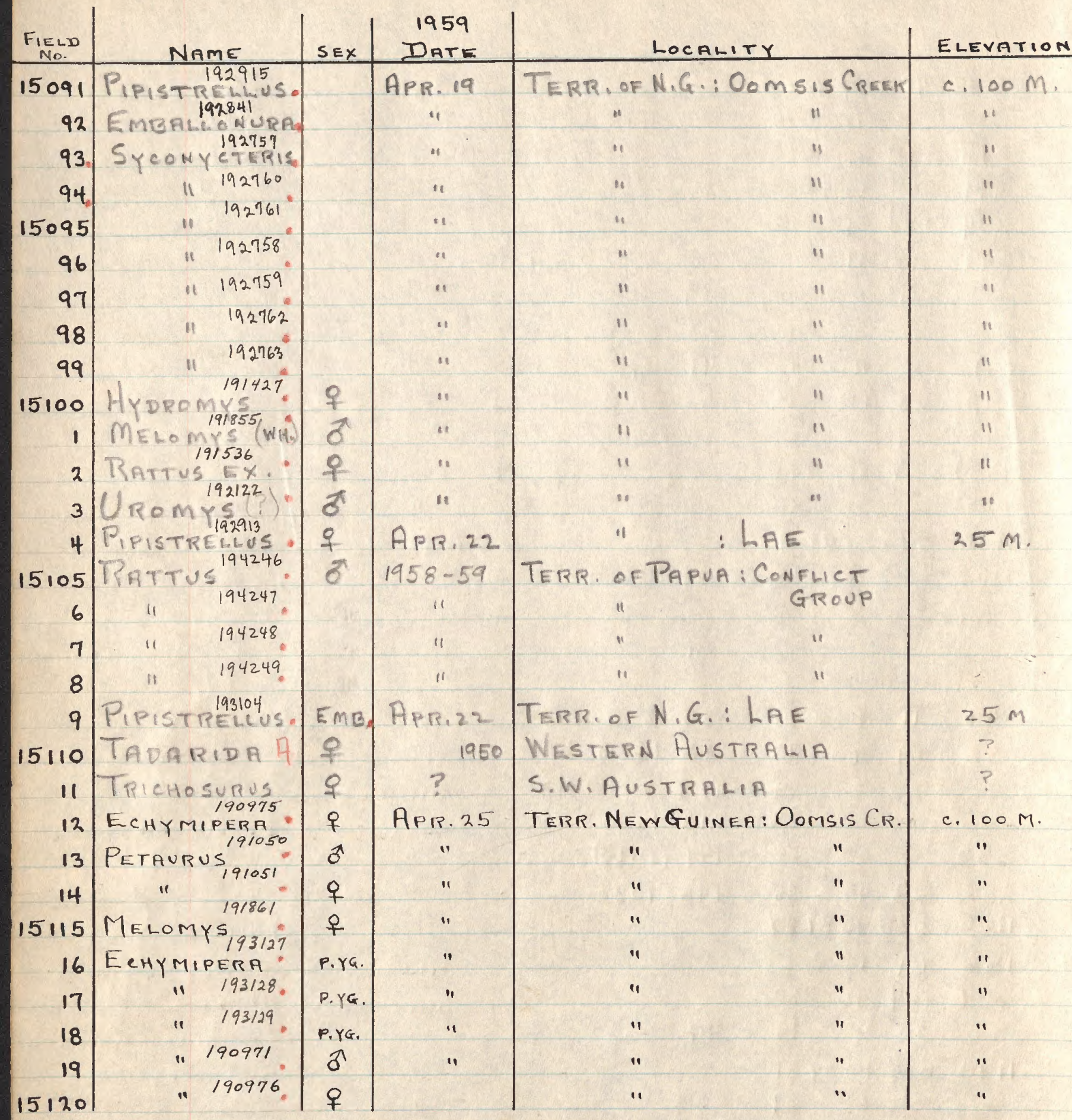


(4)

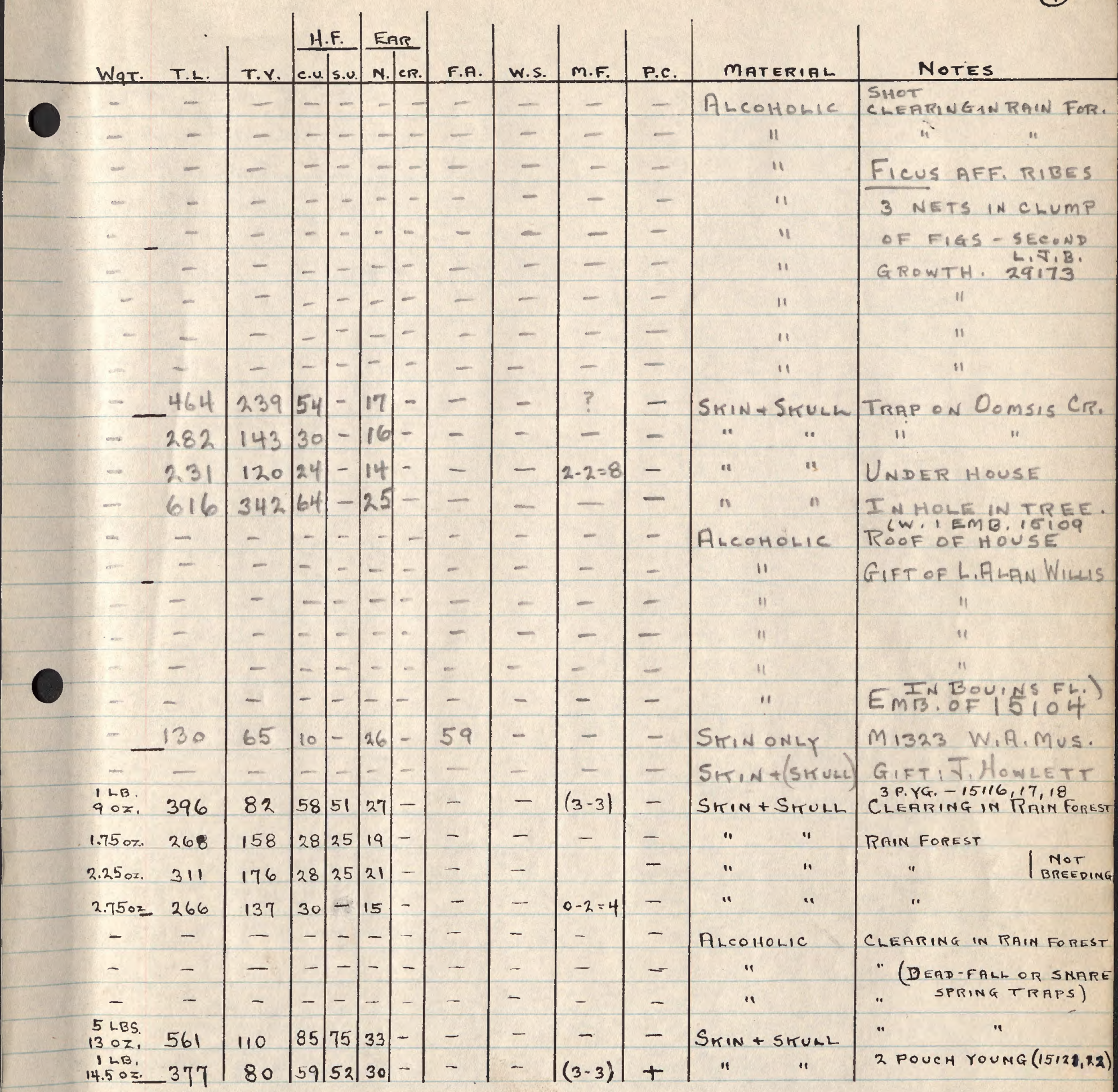

$15112,15113,15114,15115 ; 15119,15120$ BOUGHT FROM NATIVES. 


(5)

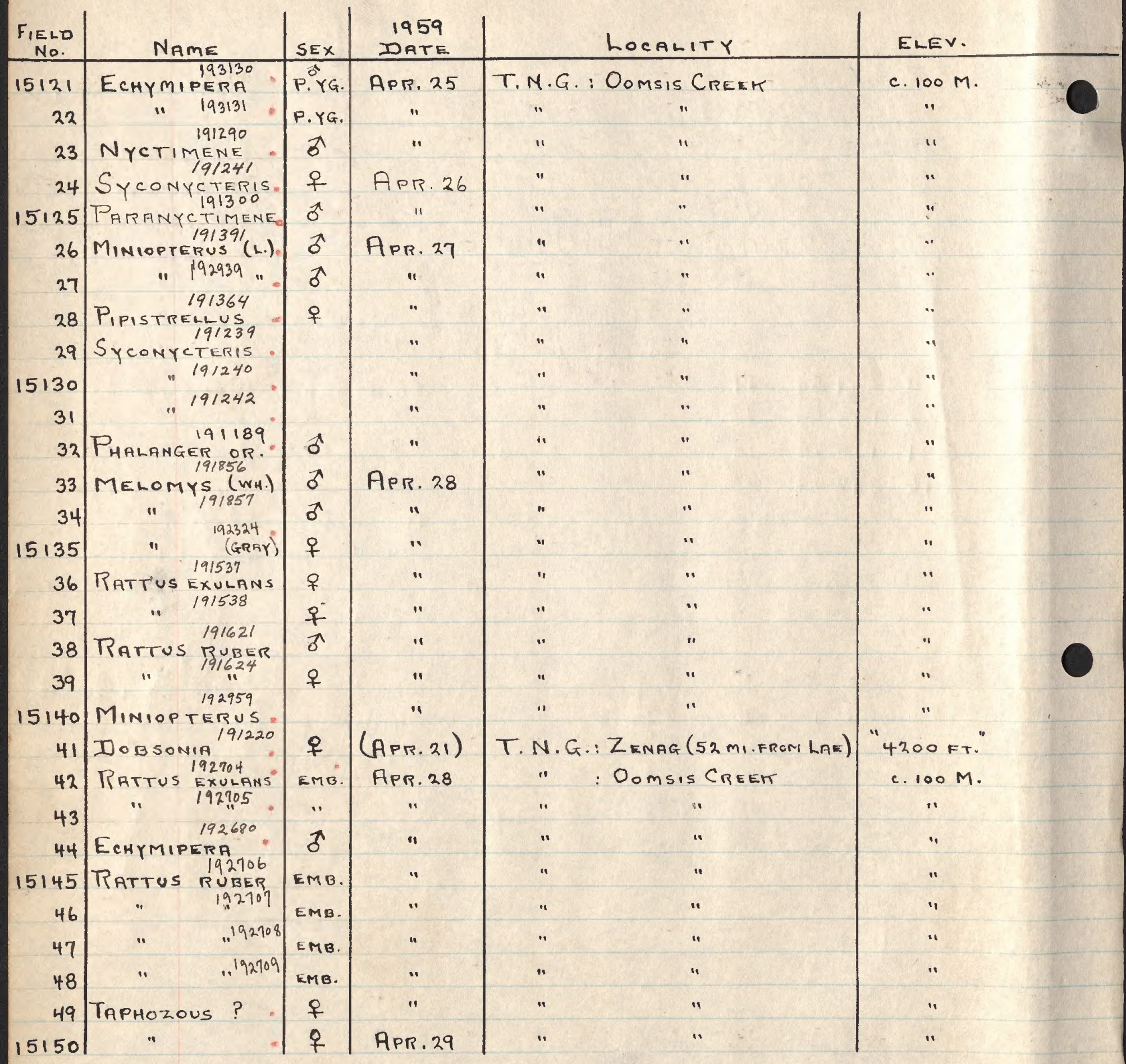

\# 15125 - ThUMB $22 \mathrm{~mm}$. SPOTS ON MEMBrane INDISTINCT YELLOWISH -WHITE SPOTS SHOW UP WELL ON PHIL. 112 OF III FINGER

150 
(5)

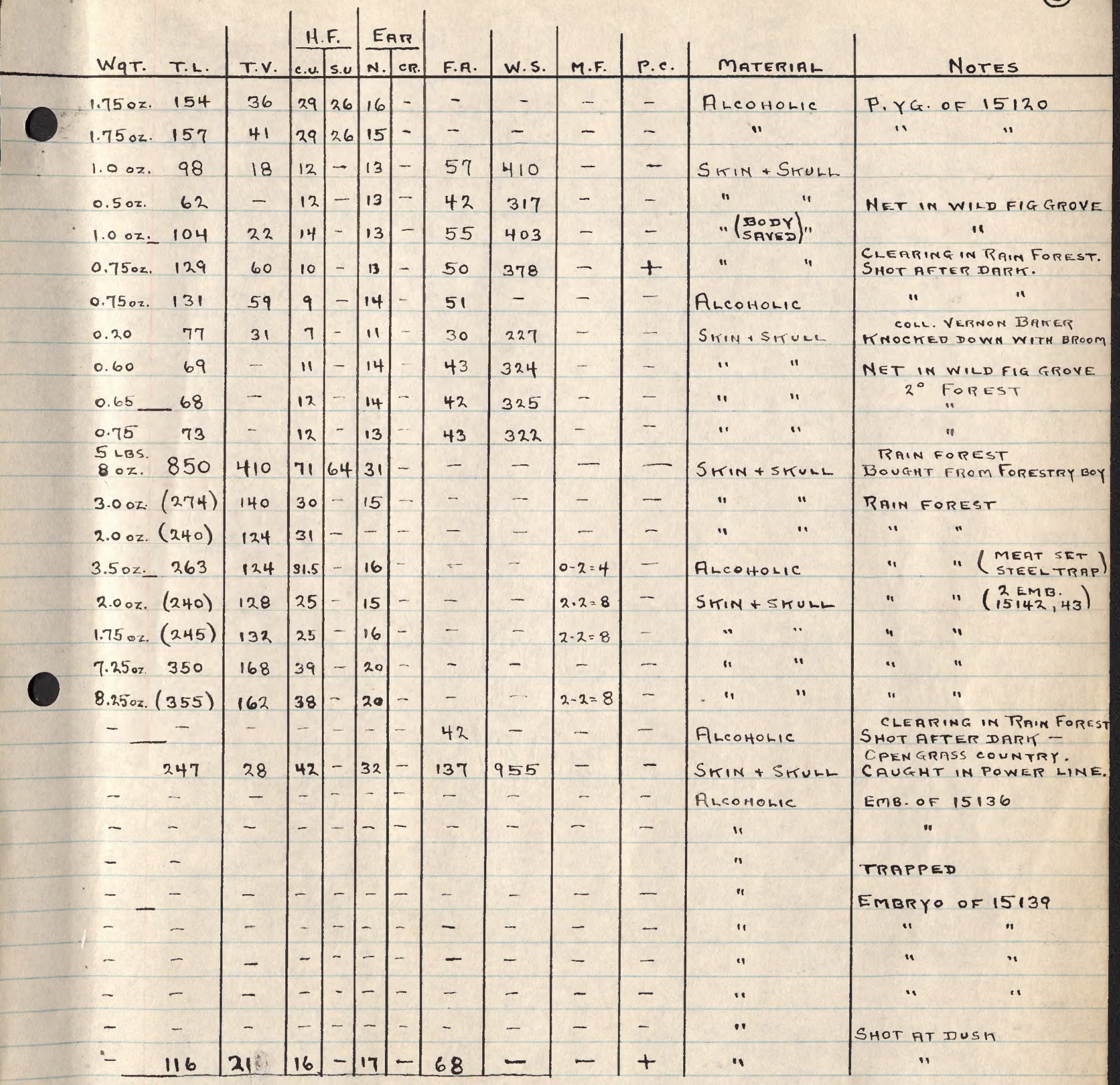


) 

(6.)

\begin{tabular}{|c|c|c|c|c|c|c|}
\hline $\begin{array}{c}\text { FIELD } \\
\text { MO. }\end{array}$ & NamE & SEX & $\begin{array}{l}1959 \\
\text { DATE }\end{array}$ & \multicolumn{2}{|c|}{ LOCALITY } & ELEY. \\
\hline $15+5.1$. & $\begin{array}{c}191306 \\
\text { EMBALSONURA } \\
190972\end{array}$ & q & APRIL 29 & T.N.G.: Oomsis & CREEK CAMP & c. $100 \mathrm{M}$. \\
\hline 52 & ECHYMIPERA & $\delta$ & $"$ & $"$ & $"$ & " \\
\hline 53 & UROMYS & $Y G . \sigma^{\pi}$ & $"$ & " & $"$ & $"$ \\
\hline 54 & MELOMYS & q & $"$ & $"$ & $"$ & $"$ \\
\hline 15155 & RATTUS RUBER & $\hat{\sigma}$ & $"$ & $"$ & $"$ & $"$ \\
\hline 56 & RatTuS EXULANS & 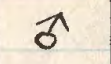 & $"$ & $"$ & " & $"$ \\
\hline 57 & " $19 / 528$ & $\delta$ & " & $"$ & $"$ & $"$ \\
\hline 58 & $\begin{array}{r}191539 \\
11\end{array}$ & 우 & $"$ & $"$ & $"$ & $"$ \\
\hline 59 & MELOMYS 191858 & $\pi$ & " & $"$ & 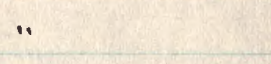 & $"$ \\
\hline 15160 & RATTUS RUBER & 우 & $"$ & $"$ & $"$ & "* \\
\hline 61 & PIPISTRELLUS & $\hat{\sigma}$ & APR. 30 & $"$ & " & $\because$ \\
\hline 62 & DOBSONIA & q & $"$ & $"$ & ZENAG & $\uparrow " \downarrow$ \\
\hline 63 & MELOMYS 191626 & 8 & $"$ & Oomsis & SCREEK CAMP & "4200 FT." \\
\hline 64 & Rattus kuber & 우 & " & $"$ & $"$ & C $100 \mathrm{M}$. \\
\hline 15165 & Rattus ExULANS & $\pi$ & " & $"$ & " & $"$ \\
\hline 66 & $" 191530$ & $\hat{\delta}$ & $"$ & $"$ & $"$ & $"$ \\
\hline 67 & $\begin{array}{l}191531 \\
191532\end{array}$ & $\hat{\sigma}$ & $"$ & $"$ & $"$ & $"$ \\
\hline 68 & 1915,32 & $\delta$ & " & $"$ & $"$ & $\because$ \\
\hline 69 & $\begin{array}{l}191320 \text { REI } \\
\text { RHINOLOPHUS }\end{array}$ & q & $"$ & " & $"$ & $"$ \\
\hline 15170 & MELOMYS 192702 & EMB. & $"$ & $"$ & " & $"$ \\
\hline 71 & 172703 & $"$ & $"$ & $"$ & $"$ & $"$ \\
\hline 72 & $\begin{array}{c}\text { TRTtUS RUBER } \\
191540\end{array}$ & q & Mry I & $"$ & " & " \\
\hline 73 & RATTUS EXULANS & 오 & $"$ & $"$ & " & " \\
\hline 74 & MELOMYS & $\sigma$ & $"$ & " & $"$ & $"$ \\
\hline 15175 & POGONOMYS & $\hat{\sigma}$ & " & $"$ & $"$ & $"$ \\
\hline 76 & " 191462 & $\delta$ & $"$ & $"$ & $"$ & “ \\
\hline 77 & 191465 & 우 & $"$ & $"$ & " & $"$ \\
\hline 78 & 191466 & ㅇ & $"$ & $"$ & "1 & " \\
\hline 79 & $\because 191463$ & juv. $\bar{\sigma}$ & MAY 2 & $"$ & " & $"$ \\
\hline $\mid 5180$ & PhILETOR & f & $"$ & $"$ & $"$ & $"$ \\
\hline
\end{tabular}

FRCHT

VIEW
NOSE

\#15169 - \#2 Markham language = Biúmpat (per hionel Baher). 
(6.)

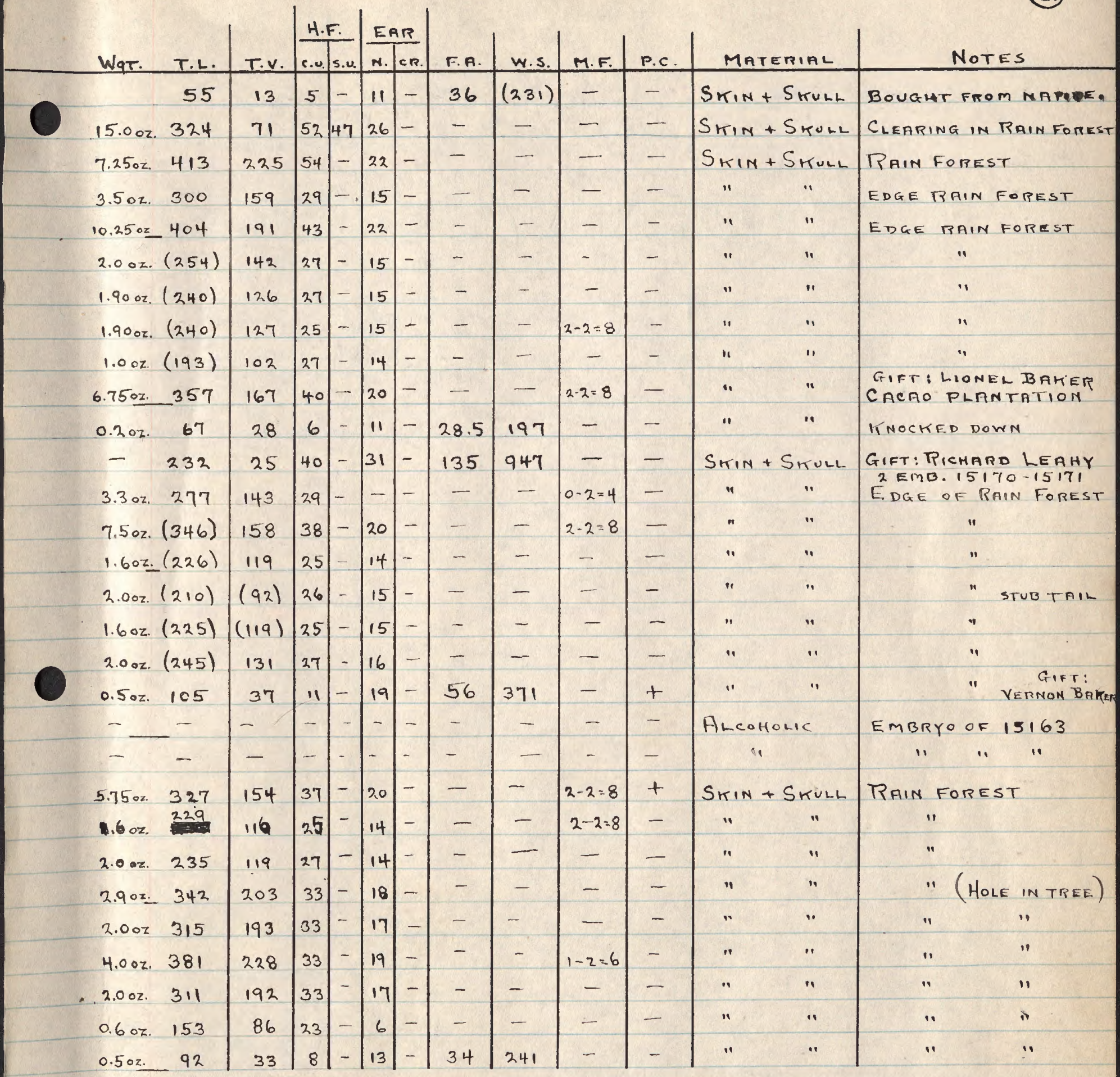

\#15180-15185, 15188 FOUND IN ROUND $(4 \times 4 \times 3)$ HOLE IN TREE (CELTIS). TAIN FOREST. PIPE HOLE INTO" NURSERY"; ABOUT ZO FEET UP, IN BOLE OF TREE (14" IN DD IA.) 



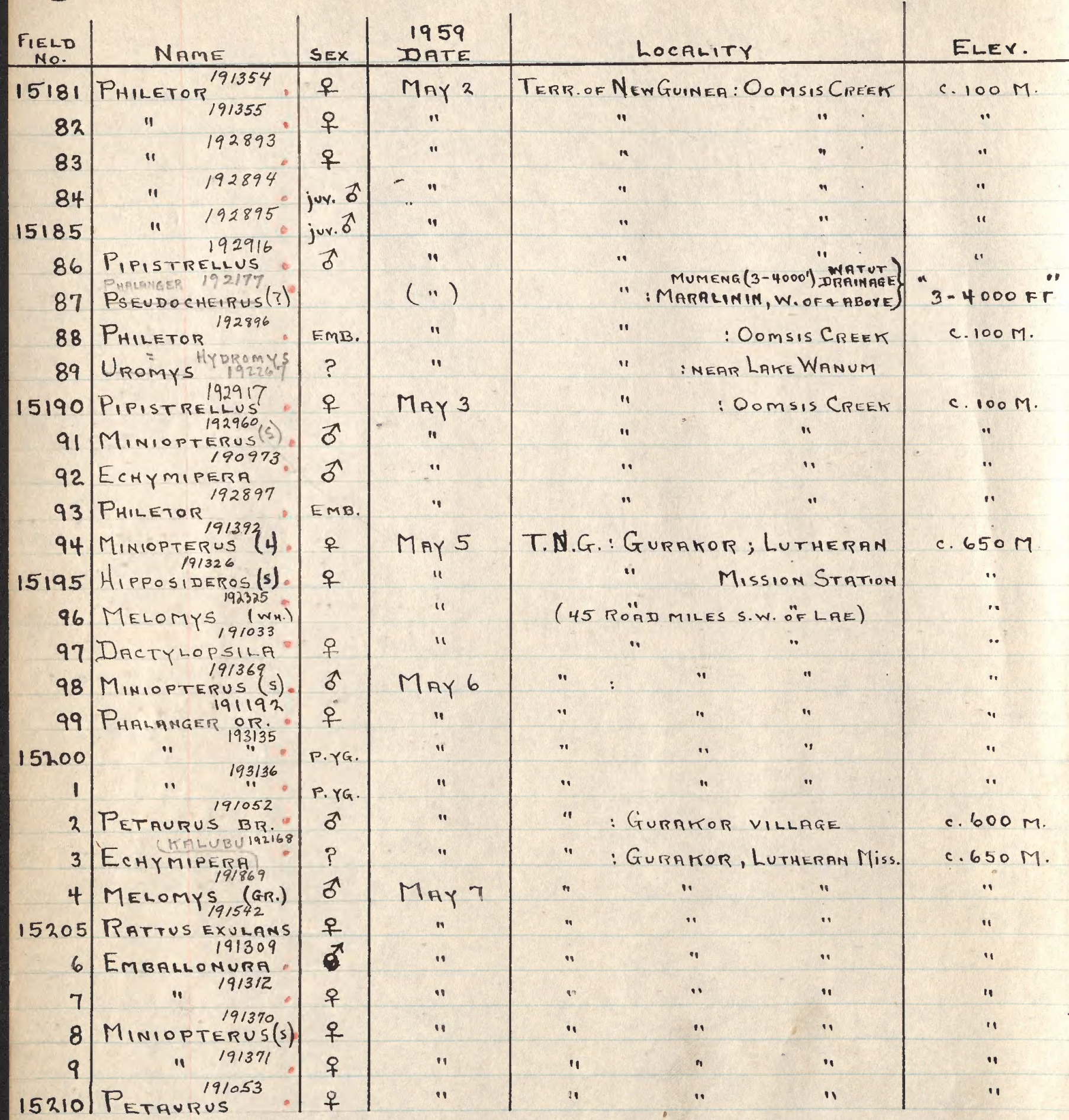




\begin{tabular}{|c|c|c|c|c|c|c|c|c|c|c|c|c|}
\hline WgT. & T.L. & T.V. & c.u. & s.u. & N. & Cr. & F.R. & w.s. & M.F. & P.c. & MATERIAL & NOTES \\
\hline$c .50 z$ & 88 & & & & & & & 237 & - & 一 & SKIN + SKULL & RAIM FOREST (HOLE IN TREE) \\
\hline $0.40 z$. & 90 & 31 & 8 & - & 12 & - & 32 & 227 & - & $一$ & $\because$ & $\because$ \\
\hline $0.40 z$ & 91 & 33 & 9 & - & 12 & - & 33 & 237 & - & - & AleONOLIC & $\because$ IEMO.(15:"93) \\
\hline - & - & - & - & - & - & - & - & - & - & - & ALCOHOLIC & " \\
\hline - & - & - & - & - & - & - & - & - & - & - & 11 & 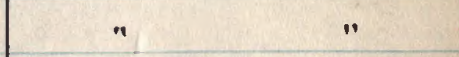 \\
\hline- & - & - & - & - & - & - & - & - & - & - & $\because$ & $\because$ \\
\hline- & 一 & - & - & - & - & - & 一 & - & - & - & $\because$ & GIFT: JOHN WOMERSLEY \\
\hline- & - & - & - & - & - & - & - & - & - & - & BOUIN'S FLUID & EMIORYO OF \#15180 \\
\hline- & - & $一$ & - & $1-$ & - & - & - & - & $一$ & - & SKULLONLY & PICK UP. FLOOD PLAIN FOREST \\
\hline 一 & $一$ & - & - & - & - & - & 29 & - & - & + & AlcoHolic & CLEARING IN RAIN FOREST \\
\hline-1 & - & - & - & - & - & - & 42 & - & - & 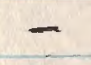 & OCTMELES & RAIN FOREST $\left\{\begin{array}{l}\text { SWARMING } \\
\text { OVER CROWN } \\
\text { OFFLOWERIMG }\end{array}\right.$ \\
\hline - & - & $(90)$ & 93 & 80 & 31 & - & - & - & - & $I$ & SKIN + SKULL & $2^{\circ}$ RRIM FOREST \\
\hline- & - & - & - & - & - & - & - & - & - & - & $\begin{array}{l}\text { ALCOHOLIC } \\
\text { (BOUINS }\end{array}$ & EMBRYO OF 15183 \\
\hline 0.502 & 125 & 55 & 10 & - & 14 & - & 51 & - & - & - & STIN + SHULL & SHOT AT DUSK. RAIN FOK \\
\hline $0.20 z$ & 82 & 29 & 8 & - & 12 & - & 48 & 310 & - & - & $"$ & SHOT 8:30 P.M. RAIN FOREST \\
\hline & - & 125 & 28 & - & - & - & - & - & & - & AR̈cOHOL & TrRAPPED. EDGE RAIN For. \\
\hline $14 \circ z$ & 597 & 332 & 48 & 43 & 29 & - & - & - & $(1-1)$ & - & SKin + S Kun & SHOT. $2^{\circ}$ RAIN FOREST \\
\hline $0.30 z$ & 97 & 45 & 8 & - & 11 & - & 43 & $(313)$ & - & + & $"$ & SHOT. EDGE OF RAIN FOR. \\
\hline $\begin{array}{l}4 \mathrm{LBS} . \\
8 \mathrm{oZ} .\end{array}$ & 760 & 357 & 59 & 54 & 26 & - & - & - & $(2-2)$ & - & " & " (w.r̈н 2 ройен үӓ.) \\
\hline 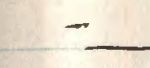 & - & $\sim$ & - & - & - & - & - & - & - & - & AlCOHOLIC & Pover YG. OF 15199 \\
\hline- & - & - & - & - & - & - & - & - & - & - & $"$ & " 4 " \\
\hline $4.750 z$ & 352 & 196 & 29 & 26 & 23 & - & - & - & - & 一 & SKIH + SKuLL & COCONUT PALMS \\
\hline- & - & - & - & - & - & - & - & - & - & - & SKUKL DNLY & THICK GRASS IN CLEARIMG \\
\hline $4.00 z$ & $(278)$ & 133 & 32 & - & $(17)$ & - & - & - & - & - & SKIN + SKULL & $2^{\circ}$ RAIN ForEST \\
\hline 1.9002 & 252 & 132 & 25 & - & 16 & - & - & - & $2-2=8$ & - & $\because$ & 11 \\
\hline$(0.15)$ & 54 & 12 & 7 & - & 12 & $\sim$ & 39 & - & - & - & " & CLEARING IN RRIN FOREST \\
\hline$(0.15)$ & 56 & 14 & 7 & - & 13 & - & 38.5 & 一 & - & - & $"$ & $\because$ \\
\hline 0.302 & 103 & 49 & 9 & - & 11 & - & $(44)$ & - & $\sim$ & - & " & EDGE OF TRIN FOREST \\
\hline $0.30 \mathrm{x}$ & 100 & 49 & 8 & - & 10 & - & 44 & 316 & - & - & “. & $\because$ \\
\hline $3.75 \mathrm{oz}$. & -318 & 173 & $30 \mid$ & $|27|$ & $23 \mid$ & - & - & - & $|(2-2)|$ & - & “ & (WITH I POUEH YG. 15211 ) \\
\hline
\end{tabular}


0

0

0 



\begin{tabular}{|c|c|c|c|c|c|c|}
\hline $\begin{array}{l}\text { FIELD } \\
\text { No. }\end{array}$ & NAME & SEK & $\begin{array}{l}1959 \\
\text { DATE }\end{array}$ & - & LOCALITY & ELEY. \\
\hline 15211 & Petaurus 193112 & of & MAY 7 & T.N.G.: & : Gurakor & c. $650 \mathrm{~m}$. \\
\hline 12 & POCONOMYS & 아 & " & 11 & OOMSIS CREEK & c. $100 \mathrm{M}$ \\
\hline 13 & $=11191468$ & \& & $"$ & "1 & $" 1$ & 11 \\
\hline 14 & $11^{191464}$ & $\hat{\sigma}$ & "1 & $" 1$ & 11 & 11 \\
\hline 15215 & " 193154 & KMBB. & $"$ & " & $"$ & $"$ \\
\hline 16 & "193155. & EMB. & " & $"$ & $"$ & $"$ \\
\hline 17 & HIPposideros (L) & & APRILI & $"$ & : REMPI, $30 \mathrm{Ml}$. N. OF & ? \\
\hline 18. & $11^{19285^{\circ}}$ & & $"$ & $"$ & Madang "Big caye & \\
\hline 19 & ${ }^{11}{ }^{192852}$ & & $"$ & " & 2 MI. LONG. WATER & \\
\hline 15220 & $\cdot 1^{192851}$ & & $"$ & $"$ & THROUGH THE WHOLE & \\
\hline 21 & "192853. & & $"$ & $"$ & TUNNEL" & \\
\hline $\begin{array}{l}22 \\
23\end{array}$ & $\begin{array}{c}192804 \\
\text { EMBALLONURA B } \\
192805 \\
\text { " } 192859\end{array}$ & & MaY 8 & T.N.G.: & $\begin{array}{c}\text { : Guramor L. Mission } \\
\text { " }\end{array}$ & $\begin{array}{c}\text { c. } 650 \mathrm{M} . \\
\end{array}$ \\
\hline 24 & HipposiderRos (s.) & & $"$ & $"$ & $"$ & " \\
\hline 15225 & Rattus ExuLans & ๘ & $"$ & $"$ & $"$ & $"$ \\
\hline 26 & Rattus Ex? & & $"$ & $"$ & " & $"$ \\
\hline 27 & ECHYMIPERA & $q$ & $"$ & " & $"$ & $"$ \\
\hline 28 & EMBALLONURA B. & ઠ & MnY 9 & $"$ & " & " \\
\hline 29 & " & 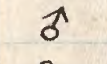 & $"$ & $"$ & $"$ & $"$ \\
\hline 15230 & MINIOPTERTUS (s) & $q$ & $"$ & $"=$ & “ & $"$ \\
\hline 31 & DOBSONIA 191221 & $\hat{\sigma}$ & $"$ & $"$ & " & " \\
\hline 32 & 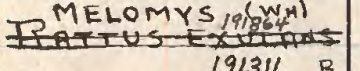 & 우 & 11 & " & $"$ & $"$ \\
\hline 33 & EMEALLONURA & ? & May 10 & 4 & $"$ & " \\
\hline 34 & " $192808^{\circ}$ & q & $"$ & $"$ & $"$ & ." \\
\hline 15235 & "192809. & & $\because$ & ." & " & $"$ \\
\hline 36 & $\because 191870^{\circ}$ & & $"$ & 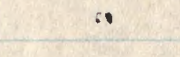 & $"$ & " \\
\hline 37 & MELOMYS $\frac{192860}{(G R)}$ & $\tilde{\sigma}$ & $"$ & $"$ & $"$ & $"$ \\
\hline 38 & HIPPOSIMEROS (s) & & 11 & 11 & 11 & " \\
\hline 39 & $\begin{array}{c}\text { EMBALLONURA } B . \\
192810\end{array}$ & & $" 1$ & 11 & 11 & " \\
\hline 40 & 11172810 & & 11 & 11 & 11 & 11 \\
\hline
\end{tabular}

EMBALLONURA, AT TIMES, USUALLY WUST BEFORE DUSH, FLIES VERY SLOWLY, 10-15 FT. HIGH, IN SWEEPING CIRCAES, WITHOUT SWOOPING OR DARTING. 


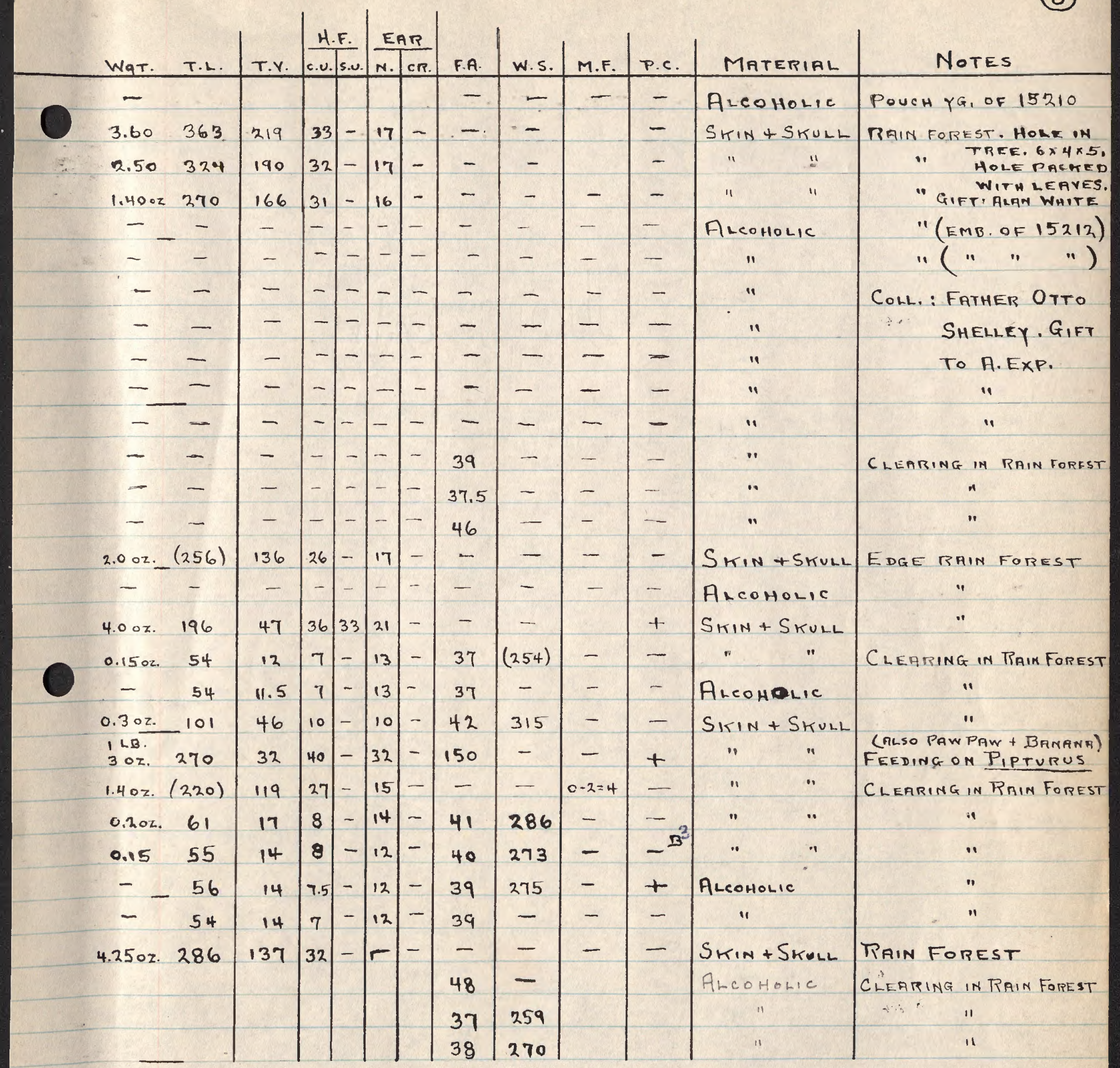




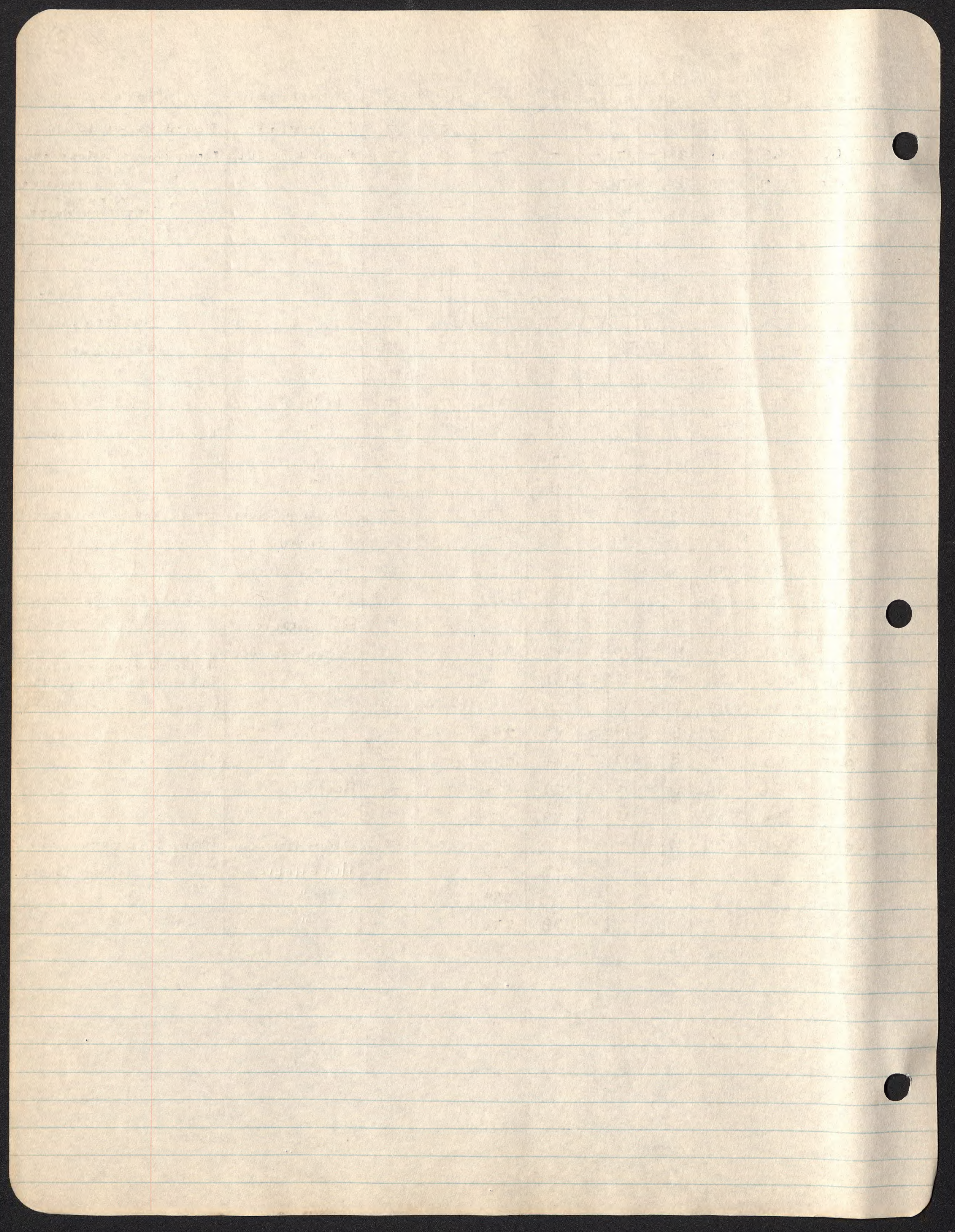



(9)

\begin{tabular}{|c|c|c|c|c|c|}
\hline $\begin{aligned} \text { FIELD } \\
\text { No. }\end{aligned}$ & NAME & SEX & $\begin{array}{l}1959 \\
\text { DATE }\end{array}$ & LOCALITY & ELEVATION \\
\hline $\begin{array}{r}15241 \\
42\end{array}$ & $\begin{array}{c}192807 \\
\text { EMBALENURA } \\
1192811\end{array}$ & & $\operatorname{MaY}_{11} 10$ & T. & c. $650 \mathrm{M}$ \\
\hline 43 & $11^{192812}$ & & "1 & $112 x+11$ & "1 \\
\hline 44 & 11192813 & & "1 & 11 & 11 \\
\hline 15245 & 11192814 & & 11 & 11 & "1 \\
\hline 46 & 11192815 & & $" 1$ & "1 & "1 \\
\hline 47 & Rattus Ex. & 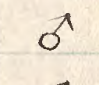 & MAY 12 & T.N.G.: KaINDI - Property of & c. $2060 \mathrm{M}$. \\
\hline 48 & " $191544 \quad "$ & 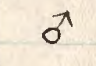 & $"$ & TOBERT FRANHLIN & " \\
\hline 49 & " 191545 " & 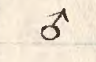 & $"$ & $"$ & $"$ \\
\hline 15250 & $\because \quad 191547$. & q & $"$ & $"$ & $"$ \\
\hline 51 & MARSUPIAL 1921668 & & $"$ & $"$ : BULLDOG TRACK $\left\{\begin{array}{l}\text { I MI. S.OF } \\
\text { EDIECREEH }\end{array}\right\}$ & c. $2100 \mathrm{M}$ \\
\hline 52 & TRATTUS $\left(G_{191546}\right)$ & f & MaY 13 & ": MAINDI & c. $2060 \mathrm{M}$. \\
\hline 53 & $" \quad x^{\prime \prime}(W H)$ & $\sigma$ & $"$ & $"$ & $"$ \\
\hline 54 & "191548 (wH) & 우 & $"$ & $" 1$ & " \\
\hline 15255 & $" 192121(G R)$ & $\pi$ & $"$ & ". & " \\
\hline 56 & " 191669 (GR) & 오 & $"$ & $"$ & " \\
\hline 57 & Rattus 191549 (WH.) & q & " & " & c. $2000 \mathrm{M}$. \\
\hline 58 & Mattus $191550\left(W 1657^{19}\right)$ & 와 & MaY 14 & " & c. $2050 \mathrm{M}$ \\
\hline 59 & " $(G R)$ & $\sigma$ & $"$ & $"$ & ". \\
\hline 15260 & $\because \quad \begin{array}{ll}191658 \\
\quad 191670\end{array}(G R)$ & $\delta$ & $"$ & $"$ & $"$ \\
\hline 61 & " $191335(G R)$ & 우 & $"$ & " & " \\
\hline 62 & PIPISTRELLUS & 오 & MaY 15 & $"$ & c. $2000 \mathrm{M}$ \\
\hline 63 & MINIODTERUS (L) & ? & $"$ & $"$ & $"$ \\
\hline 64 & Rattus & 오 & " & $"$ & c. $2060 \mathrm{M}$. \\
\hline 15265 & $" 191552\left(W_{H}\right)$ & 오 & $"$ & $"$ & $"$ \\
\hline 66 & " $191502(W H)$ & 우 & $"$ & " & $"$ \\
\hline 67 & $\Rightarrow \quad 191554(W(14)$ & 오 & " & " & $"$ \\
\hline 68 & $191555(w h)$ & $\$$ & $"$ & $"$ & $"$ \\
\hline 69 & $\begin{array}{l}191557 \text { (WH) } \\
191450\end{array}$ & $\hat{\sigma}$ & $"$ & $"$ & ? \\
\hline 15270 & ANISOMYS & $\delta$ & MAY 16 & MT. " & c. $2200 \mathrm{M}$ \\
\hline
\end{tabular}




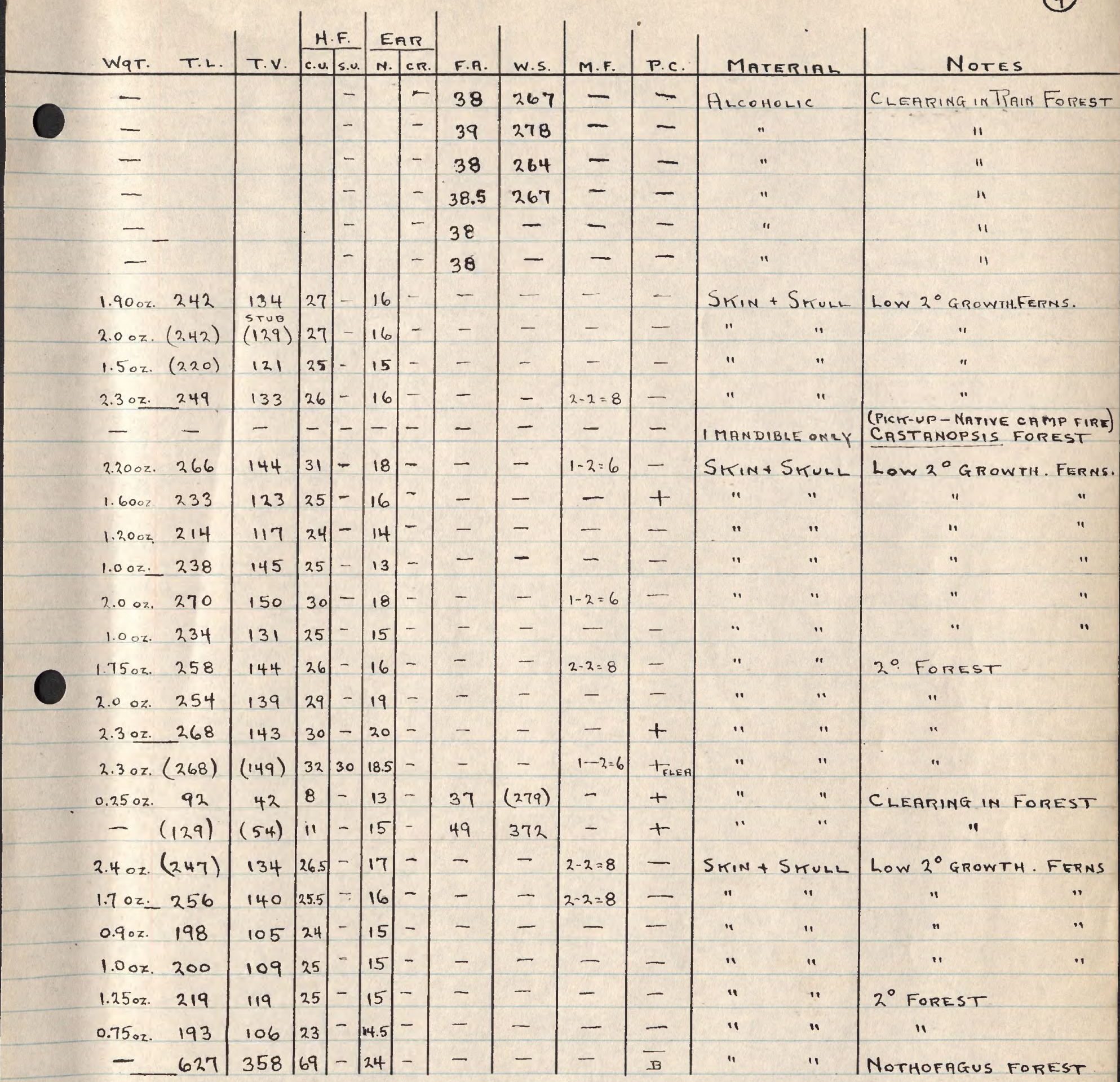




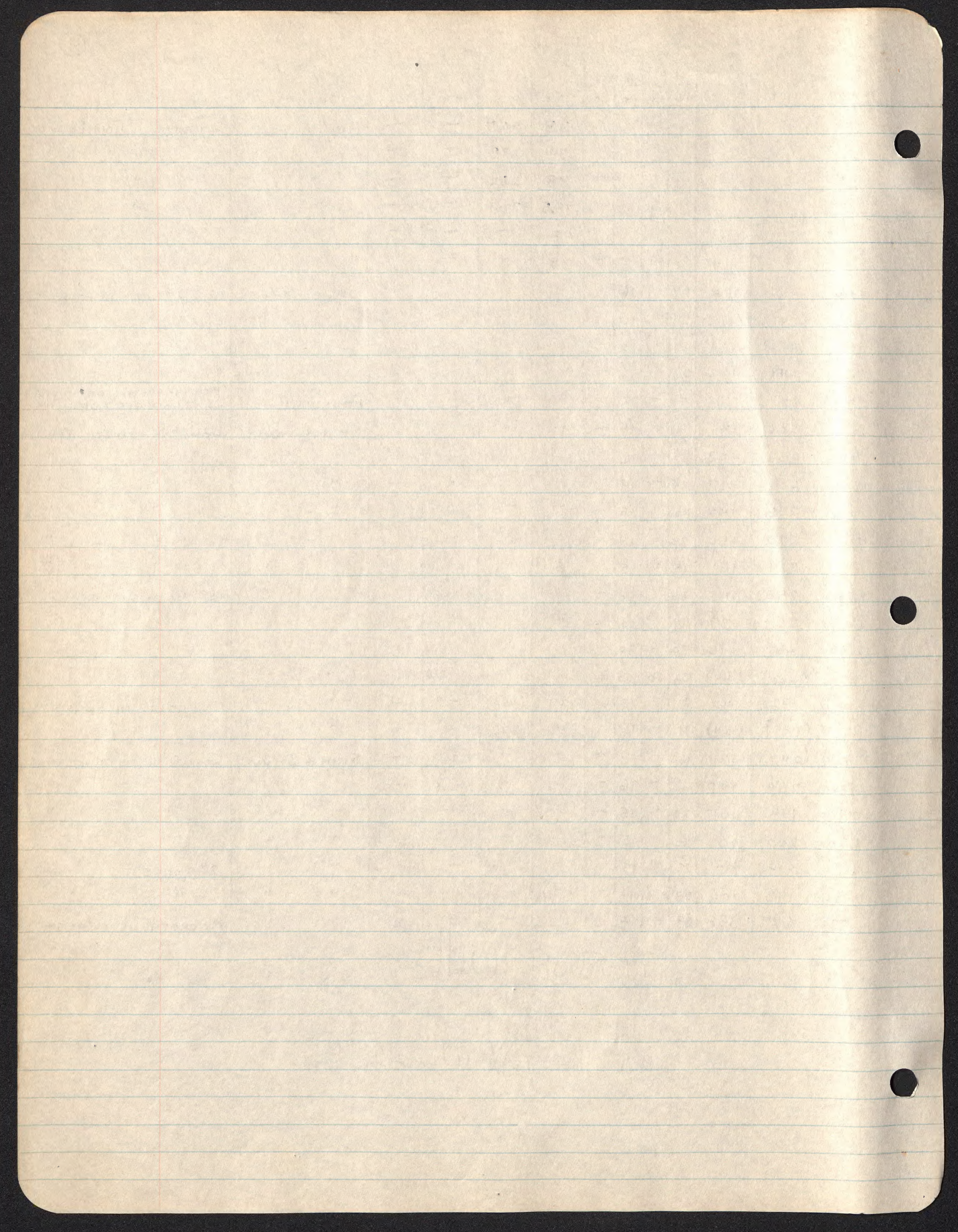





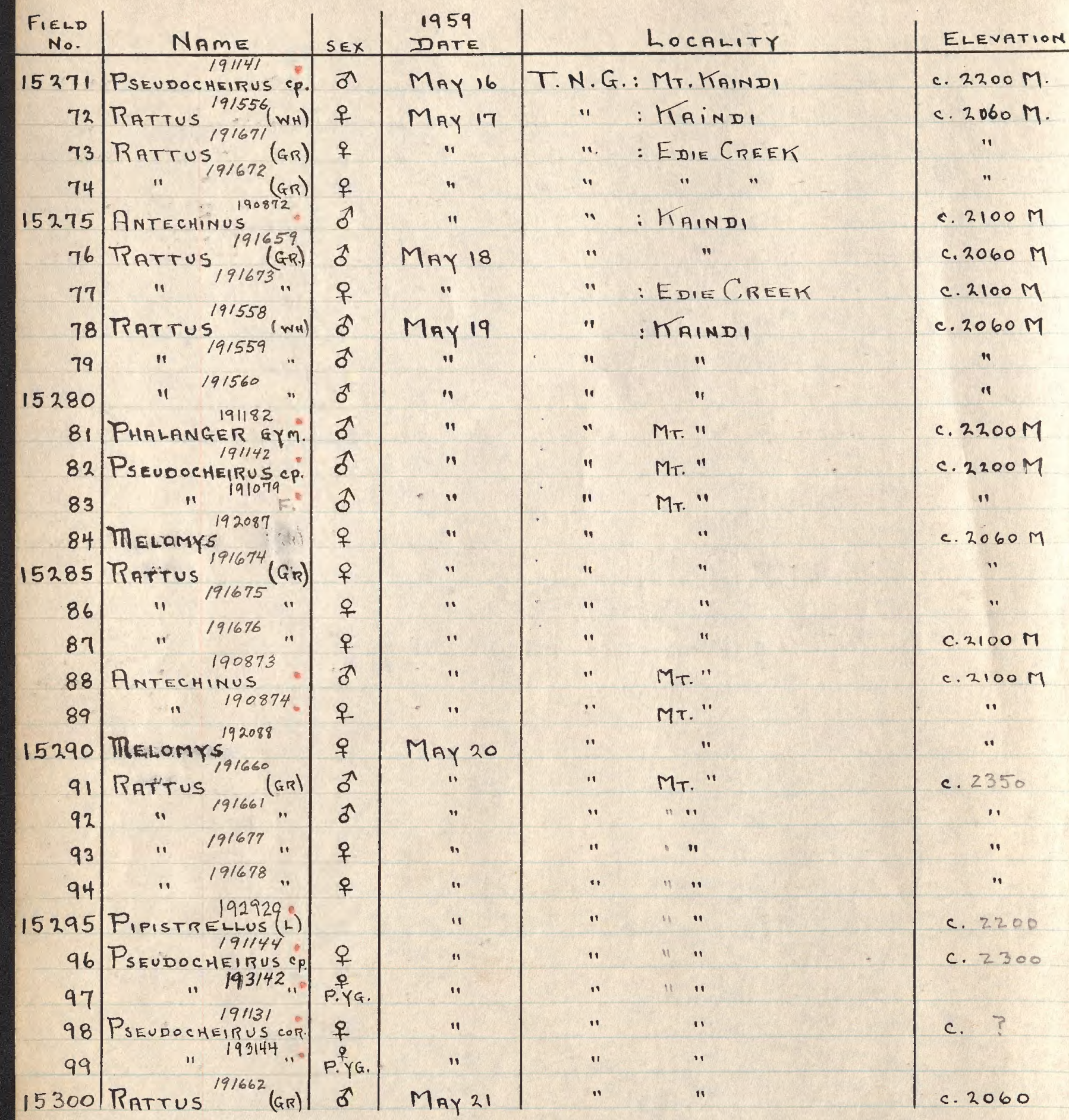

15288,89 TRAPPED ALONG BEO OF VERY SMAGE STREAM ON MT. MAINDI 
(10)

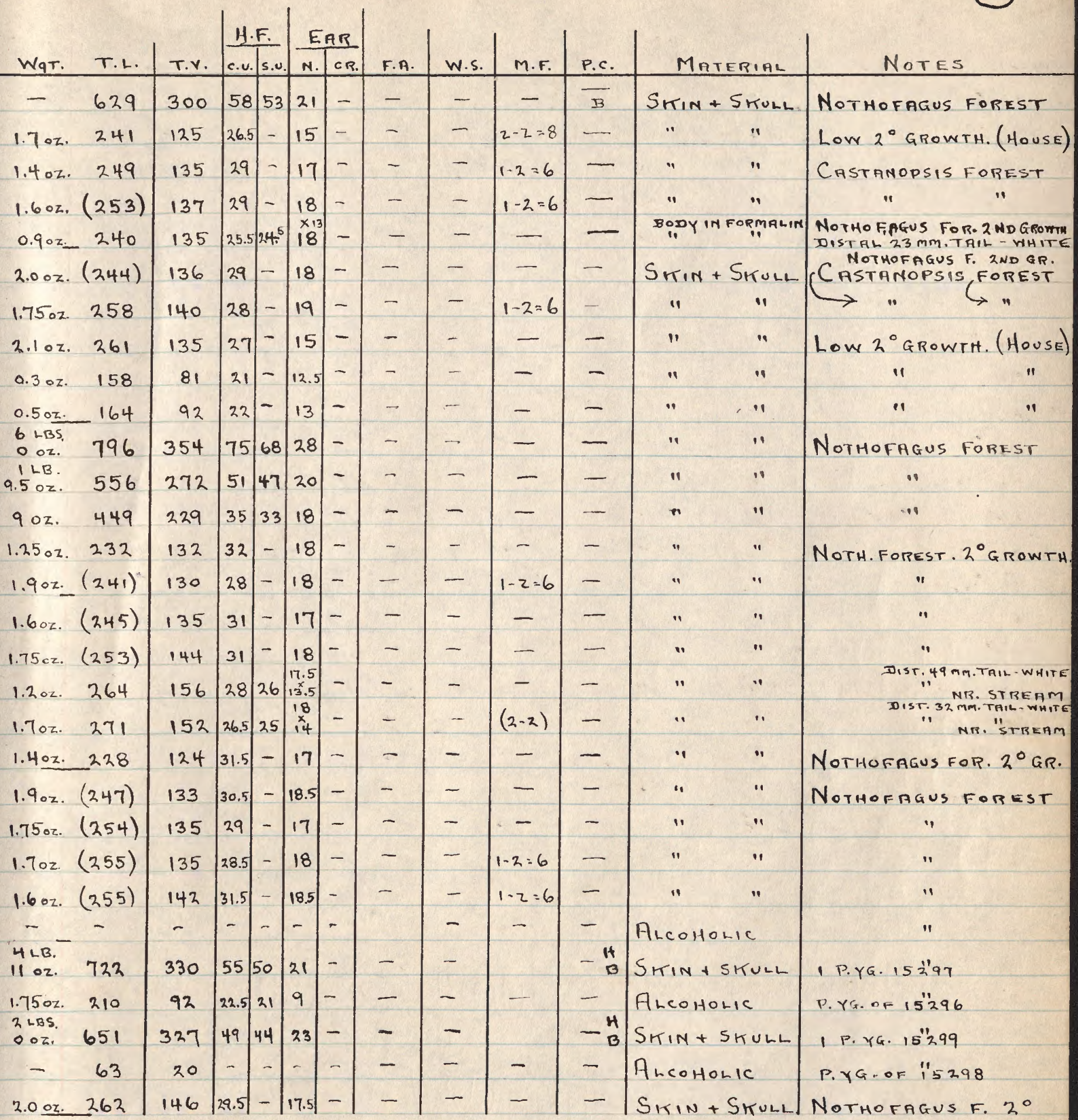

$$
\begin{aligned}
& 15296 \text { - HEART WEIGHT }=0.20 \mathrm{Z} . \\
& 15298 \text { - " " }
\end{aligned}
$$





\begin{tabular}{|c|c|c|c|c|c|}
\hline $\begin{array}{l}\text { FiELD } \\
\text { NO. }\end{array}$ & NAME & SEX & $\begin{array}{l}1959 \\
\text { DATE }\end{array}$ & LOCALITY & ELEV. \\
\hline 15301 & Rattus 191679 & 아 & MaY 21 & T.N.G.: KAINDI & c. $2060 \mathrm{M}$. \\
\hline 2 & Miniopterus & $\widehat{\sigma}$ & " & " & c. $2100 \mathrm{M}$. \\
\hline 3 & " 191374 & $\hat{\sigma}$ & $"$ & $"$ & $"$ \\
\hline 4 & 11 191375 & 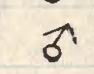 & $"$ & " & $"$ \\
\hline 15305 & "19/376 & 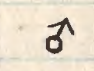 & $"$ & " & " \\
\hline 6 & $\because \quad 192961$ & $\hat{\sigma}$ & " & " & $"$ \\
\hline 7 & $11 \quad 192962$ & $a$ & $"$ & $"$ & $"$ \\
\hline 8 & "192963. & $\hat{\sigma}$ & $"$ & $"$ & " \\
\hline 9 & " 191378 & $q$ & " & $"$ & $"$ \\
\hline 15310 & "191379. & 우 & $"$ & $"$ & $\because$ \\
\hline 11 & 11 191380 & t & $"$ & " & " \\
\hline 12 & "192964 & 운 & " & ". & $"$ \\
\hline 13 & "192965. & 우 & $"$ & $"$ & $"$ \\
\hline 14 & 11 192966. & 오 & $"$ & $"$ & $"$ \\
\hline 15315 & 11 192967 & 오 & $"$ & $"$ & $"$ \\
\hline 16 & " $\quad 192968$ & 오 & $"$ & $n$ & $"$ \\
\hline 17 & 192969 & q & $"$ & $"$ & $"$ \\
\hline 18 & 192970 & $q$ & $"$ & $"$ & $"$ \\
\hline 19 & 1" 192971 & 우 & $n$ & " & $"$ \\
\hline 15320 & Ratrus ${ }^{19 / 680}\left(G_{R}\right)$ & q & $"$ & $"$ & $"$ \\
\hline 21 & MELOMYS 192083 & $\pi$ & MaY 22 & $"$ & c. $2100 \mathrm{M}$. \\
\hline 22 & $11 \quad 192089$ & 웅 & " & $"$ & $"$ \\
\hline 23 & 192084 & $\hat{2}$ & " & $"$ & " \\
\hline 24 & " 192085 & $\pi$ & " & " & " \\
\hline 15325 & RATTUS 191663 & $\delta$ & " & $"$ & $"$ \\
\hline 26 & $\begin{array}{c}191195 \\
\text { Phalanger VEST: } \\
191042\end{array}$ & $\hat{0}$ & MaY 23 & 4 mi." :UPPER WATUT RIVER FALL & \\
\hline 27 & DACTYLONAX & q & $n$ & $\begin{array}{l}\text { 2MI." (ERSTERN DRAINAGE) } \\
4,2,3 \text { MILES W.N.W.OF }\end{array}$ & c. $2200 \mathrm{M}$. \\
\hline 28 & MELOMYS (2) 192090 & 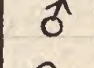 & " & 3 MI. " KaINDI, JUNCTION OF EDIE + MAERI R & IVERS \\
\hline 29 & MELOMYS (1) & 오 & " & ": TRINDI & c. $2100 \mathrm{M}$ \\
\hline 15330 & $\quad 11 \quad(1)^{192041}$ & 우 & $"$ & “ & " \\
\hline
\end{tabular}

15302-15319 TAKEN IN GOLD MINE TUNNEL (D̈BIVE") 


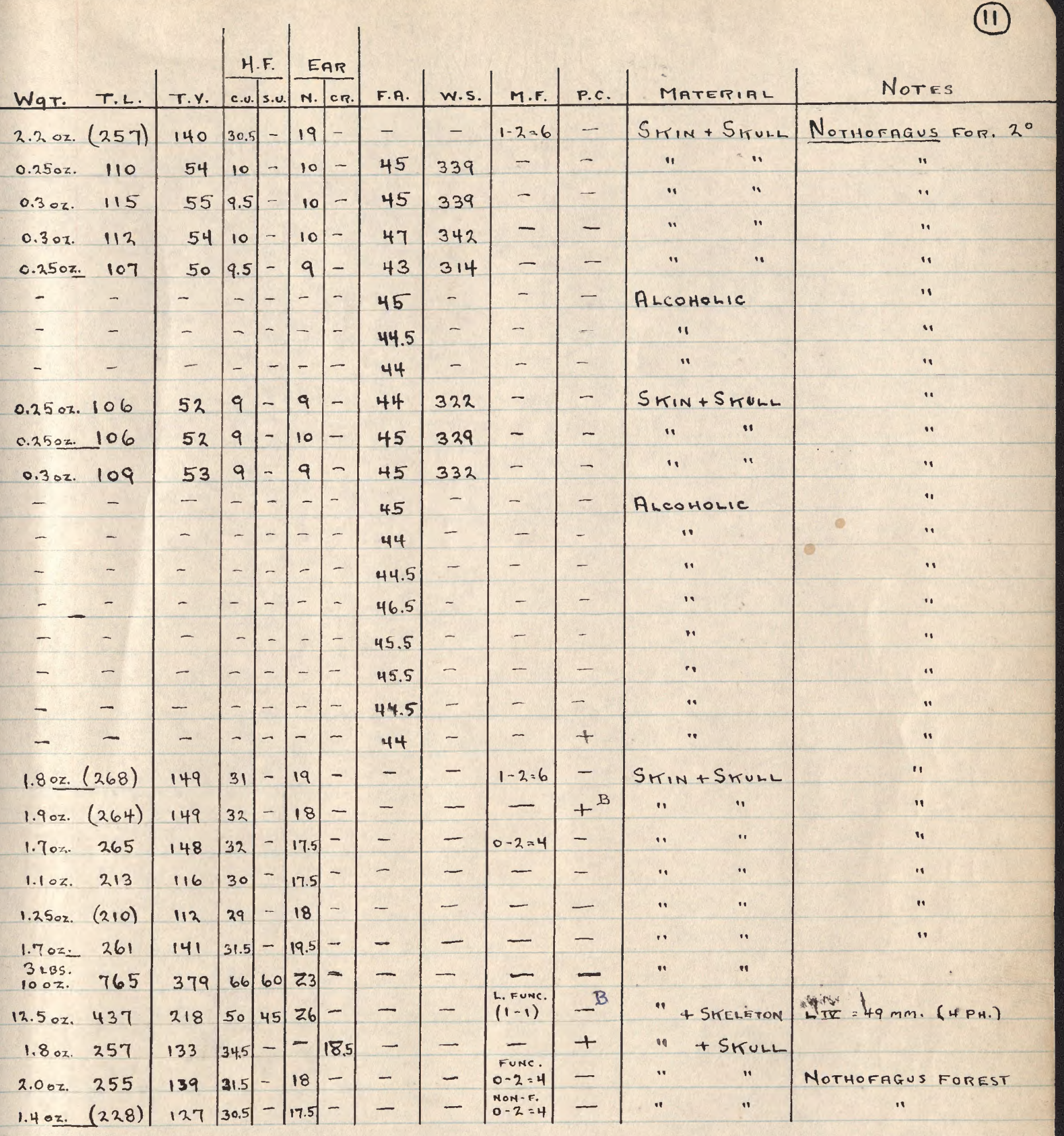





\begin{tabular}{|c|c|c|c|c|c|}
\hline $\begin{aligned} \text { EIELD } \\
\text { No. }\end{aligned}$ & NAME & SEX & DAtE & LOCALITY & ELEV. \\
\hline 15331 & ThtTUS 191664 (GR) & $\delta$ & MaY 23 & T.NG.: KRINDI & c. $2100 \mathrm{M}$. \\
\hline 32 & " 191665 " & $\delta$ & $"$ & " & " \\
\hline 33 & " 191666 " & $\delta$ & " & $"$ & $"$ \\
\hline $\begin{array}{r}34 \\
15335\end{array}$ & $\begin{array}{r}192764 \\
\text { SYCONYCTERIS } \\
11 \quad 192765 \\
\quad 192993\end{array}$ & $\frac{0}{6}$ & $\begin{array}{c}\text { MaY } \\
\text { " }\end{array}$ & $\begin{array}{l}\text { " Bulolo, Sawmill Creeir } \\
\text { " logging area }\end{array}$ & $\begin{array}{c}3500 \mathrm{FT} . \\
. "\end{array}$ \\
\hline 36 & PHONISCUS & $\pi$ & $"$ & $"$ & 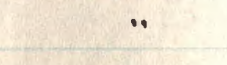 \\
\hline 37 & $\begin{array}{l}191336 \\
\text { PIPISTRELLUS }\end{array}$ & q & May 24 & ": Mr.KÍINDI & c. $2300 \mathrm{M}$ \\
\hline 38 & PSEUDOCHEIRUS & $\hat{\sigma}$ & $"$ & $"$ & c. $2200 \mathrm{M}$ \\
\hline 39 & TATtuS (GR.) & 우 & $"$ & $\because:$ : KAINDI & c. $2100 \mathrm{M}$ \\
\hline 15340 & " 191682 " & 오 & 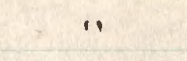 & " & “ \\
\hline 41 & $\begin{array}{l}191508 \\
\text { LORENT } 21916 Y 5\end{array}$ & $\sigma$ & $"$ & $"$ & " \\
\hline 42 & Rattus (GR) & ๘ & MaY 25 & $"$ & " \\
\hline 43 & HYomYs, 191164 & $\hat{\sigma}$ & * " & : MT. KIFINDI & c. $2200 \mathrm{M}$. \\
\hline 44 & Dorcopsulus & $\delta$ & $"$ & ": NEAR BULLDOGTRACK" & ?c. $2300 \mathrm{M}$ \\
\hline 15345 & PhalangER VEST. & 오 & $"$ & " (POSSIBLY 10-12 MILES & $"$ \\
\hline 46 & PSEUDOCHEIRUS & q & " & " SOUTH OF TÍAINDI) & " \\
\hline 47 & PSEUDOCHEIRUS & $\hat{\sigma}$ & $"$ & $\because$ & $"$ \\
\hline 48 & "19183 eup & ๘ & $"$ & $"$ & " \\
\hline 49 & DENDROLAGUS & ? & $(n)$ & $"$ : WaU (WICINITY of) & $?$ \\
\hline 15350 & ProtemnodoN? ? & $440 ?$ & $(")$ & " (POSSÏBLY MTS. N. OF WAU ) & $?$ \\
\hline $\begin{array}{l}51 \\
52\end{array}$ & $\begin{array}{l}\text { PARAHYDROMYS } \\
\text { PERI } \\
\text { DENDROLAGUS } \\
191081\end{array}$ & के & MaY 26 & 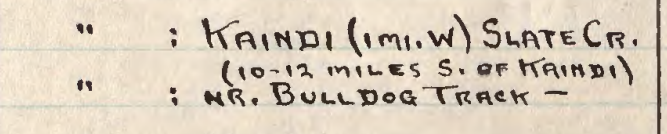 & $\begin{array}{l}\text { c. } 2000 \mathrm{M} \\
\text { c. } 2300 \mathrm{M}\end{array}$ \\
\hline 53 & PSEUDOCHEIRUS (BR) & 온 & MaY 27 & : MT. KAINDI & c. $2200 \mathrm{M}$. \\
\hline 54 & MINIOPTERUS $_{192972}(s)$ & $\hat{\sigma}$ & $"$ & : KAINDI- 2 MI.W.N.W. & c. $2100 \mathrm{M}$. \\
\hline 15355 & "1 $192972=$ & $\hat{\sigma}$ & $"$ & $"$ & " \\
\hline 56 & (9) & $\hat{\sigma}$ & $"$ & " & " \\
\hline 57 & 192974. & $\hat{o}$ & " & $"$ & $"$ \\
\hline 58 & $192975^{\circ}$ & $\hat{\delta}$ & $"$ & " & " \\
\hline 59 & 192916 。 & $\delta$ & $"$ & $"$ & " \\
\hline 153601 & 192977 & $\hat{\sigma}$ & $"$ & $" 1$ & $"$ \\
\hline
\end{tabular}

15344-15348: COLLECTED BY NATIYE BOSS BOY (WITH DOG) (FROM GOLD CAMP OF MR.

15343 : POSSIBLY SNARED BY BOY FROM SAME CAMP

15351: Locality - possibly "State CreEk" (Branch of Edie Creek) (SEE GEOG. Journal-NOY.1934-ChInNERY + SPINKS) 


\begin{tabular}{|c|c|c|c|c|c|c|c|c|c|c|c|c|}
\hline \multirow[b]{2}{*}{ Wat. } & \multirow[b]{2}{*}{ T.L. } & \multirow[b]{2}{*}{ T.V. } & \multicolumn{2}{|c|}{ H.F. } & \multicolumn{2}{|c|}{ EAR } & \multirow[b]{2}{*}{ F.A. } & \multirow[b]{2}{*}{ W.s. } & \multirow[b]{2}{*}{ M.F. } & \multirow[b]{2}{*}{ P.C. } & \multirow[b]{2}{*}{ MATERIAL } & \multirow[b]{2}{*}{ NOTES } \\
\hline & & & c.u. & s.u. & $N$. & CR. & & & & & & \\
\hline $2.7 \mathrm{oz}$ & 285 & 157 & 31.5 & - & 21 & - & - & - & - & - & SKIN + SKULL & NOTHOFRGUS FOREST \\
\hline 2.102 & 252 & 135 & 29 & - & 18 & - & - & - & - & - & $\because$ & $\because$ \\
\hline $2.00 z$ & 273 & 157 & 30.5 & - & 18 & - & - & - & - & - & " & $"$ \\
\hline- & - & - & - & - & - & - & & - & - & - & AlCOHOLIC & DIZY, SUBMONTAME \\
\hline- & - & - & - & - & - & - & & - & - & - & $"$ & FOREST: ARAUCARIA \\
\hline- & - & - & - & - & - & - & & - & - & - & $\because$ & DOMINATED." \\
\hline $0.150 z$ & 90 & 41 & 8 & - & 12 & - & 38 & $(268)$ & - & - & SKIN + SKULL & NOTHOFAGUS FOREST \\
\hline $14.10 z$ & 499 & 253 & 39 & - & 35 & - & $\ldots$ & - & - & $-B$ & ( & " \\
\hline $2.0 \mathrm{oz}$ & 271 & 148 & 30 & - & 20 & - & - & - & $1-2=6$ & - & " & $2^{\circ}$ \\
\hline $1.60 \mathrm{~m}$. & 254 & 140 & 29.5 & - & 18 & - & - & - & $1-2=6$ & $\rightarrow$ & " $\quad$ " & $2^{\circ}$ \\
\hline $0.50 z$ & 197 & 123 & 24.5 & - & $\begin{array}{l}17 \\
x \\
9\end{array}$ & - & $\sim$ & - & - & $-B$ & " +SKELË̈H. & $" 1$ \\
\hline $1.5 \mathrm{oz}$ & $(239)$ & 142 & 30 & - & 18 & - & - & - & - & - & $" \quad "$ & $2^{\circ}$ \\
\hline $\begin{array}{l}4 \text { LBS. } \\
0 \text { oz. }\end{array}$ & 786 & 407 & 79 & 71 & 28 & - & 一 & - & - & - & $\because$ & $n$ \\
\hline $\begin{array}{l}5 L B S \\
12.0 Z\end{array}$ & 745 & 320 & 112 & 100 & 39 & - & - & - & - & - & 11 & $? \quad 1$ \\
\hline $\begin{array}{l}\text { 6LBS. } \\
\text { OOX. }\end{array}$ & 850 & 411 & 70 & 61 & 24 & - & - & - & & $-B$ & + "Bones " & $? \quad 1 \%$ \\
\hline $\begin{array}{l}4 \text { LBS. } \\
50 z \text {. }\end{array}$ & 737 & 333 & & & 20 & - & - & - & & & " & $?$ \\
\hline $\begin{array}{l}2 L B S \\
70 Z .\end{array}$ & 640 & 314 & 55 & 44 & 21 & & & & + & & 14 & ? \\
\hline $\begin{array}{l}3 \text { LBS. } \\
11 \mathrm{OZ} .\end{array}$ & 721 & 324 & & & 23 & - & - & - & & - & " & $? \quad \cdots$ \\
\hline- & 一 & - & - & - & - & - & - & - & - & - & SKULL (PARTIAL) & $?$ \\
\hline- & - & - & - & - & - & - & - & - & - & - & " & ? \\
\hline $3.00 z$ & 313 & 173 & 39 & - & 13 & - & - & - & - & $\underline{B(2)}$ & SKIN + SKULL & $\begin{array}{l}\text { MT.BROOK. } \\
\text { ALONG CLEAR-FLONING }\end{array}$ \\
\hline$*$ & - & - & - & - & - & - & - & - & 一 & - & SKULL + I LEG & NOTHOFRGUS FOREST? \\
\hline $9.10 z$ & 454 & 227 & 35 & 31 & 18 & - & 一 & - & 一 & - & SKIN + SKUML & $" 1$ \\
\hline $0.20 z$ & 111 & 55 & 9 & - & 10 & - & $\begin{array}{l}46 \\
45.5\end{array}$ & 338 & - & + & AlCOHOLIC & $\begin{array}{c}\text { Castanopsis } \\
\text { MINETUNNEL Forrest } \\
\text { " }\end{array}$ \\
\hline & & & & & & & 45.5 & & & & " & "1 \\
\hline & & & & & & & 46.5 & & & & $"$ & " \\
\hline & & & & & & & 45.5 & & & & " & " \\
\hline & & & & & & & 46 & & & & " & " \\
\hline & & & & & & & 44.5 & & & & "1 & $" 1$ \\
\hline
\end{tabular}

\footnotetext{
* 15352 : "Est. WqT. about 70 Les., Length about 7 feet. Generally black, WITH A DARK RED RUMP; AVERY STRONG HERD WHICH IS BLACK. HORACE (HORrie") WILfred Clissold 15354-15367: IN GOLD MINE DRIVES (POST WAR) PUT IN BY H.W. CLISSOLD. BATS HUNG SINGLY, WINGS BENT + TRAPED IN FRONT, INRCTIYE, NOME FLEW WHEN FPPRORTHED.
} 



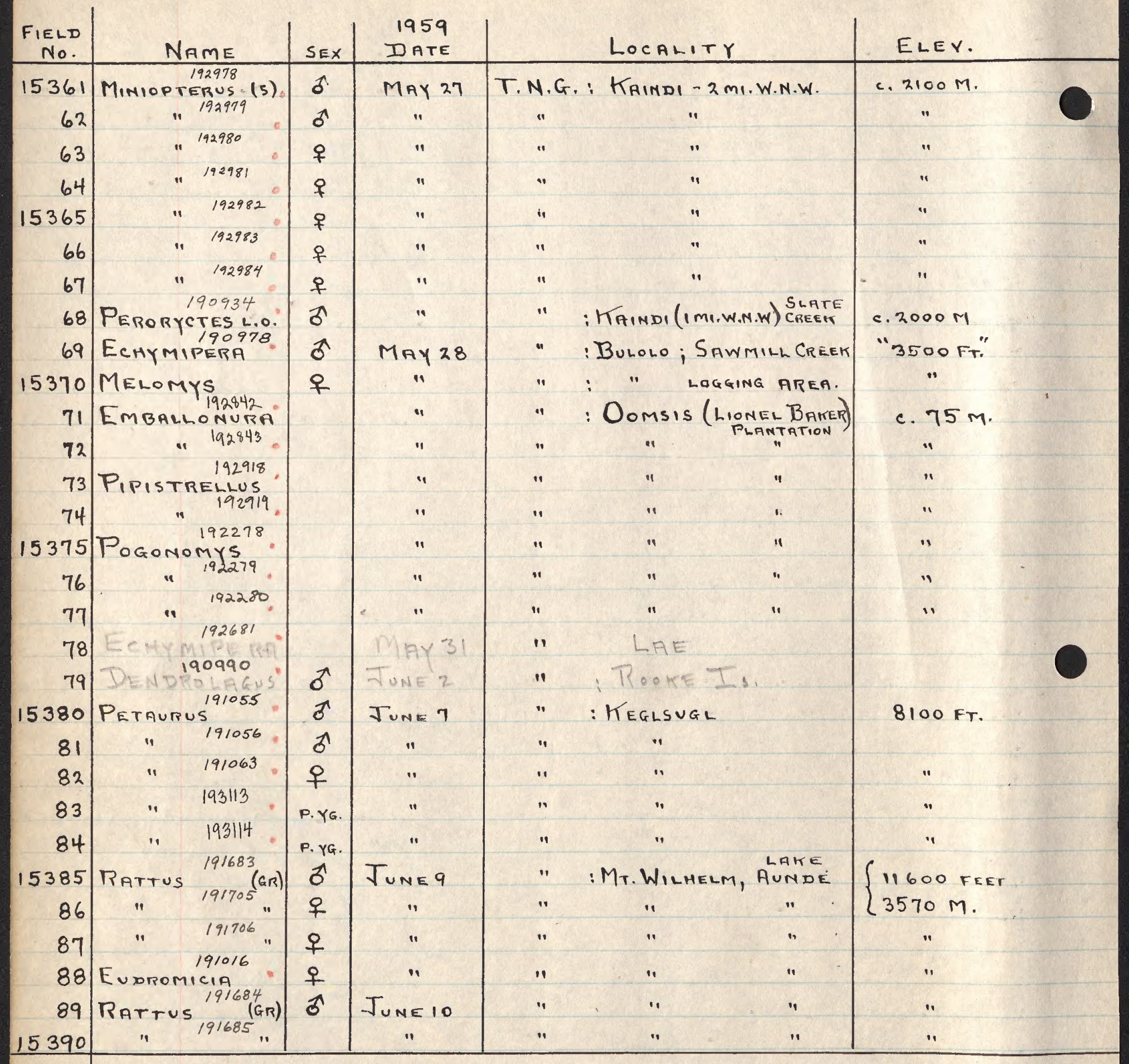




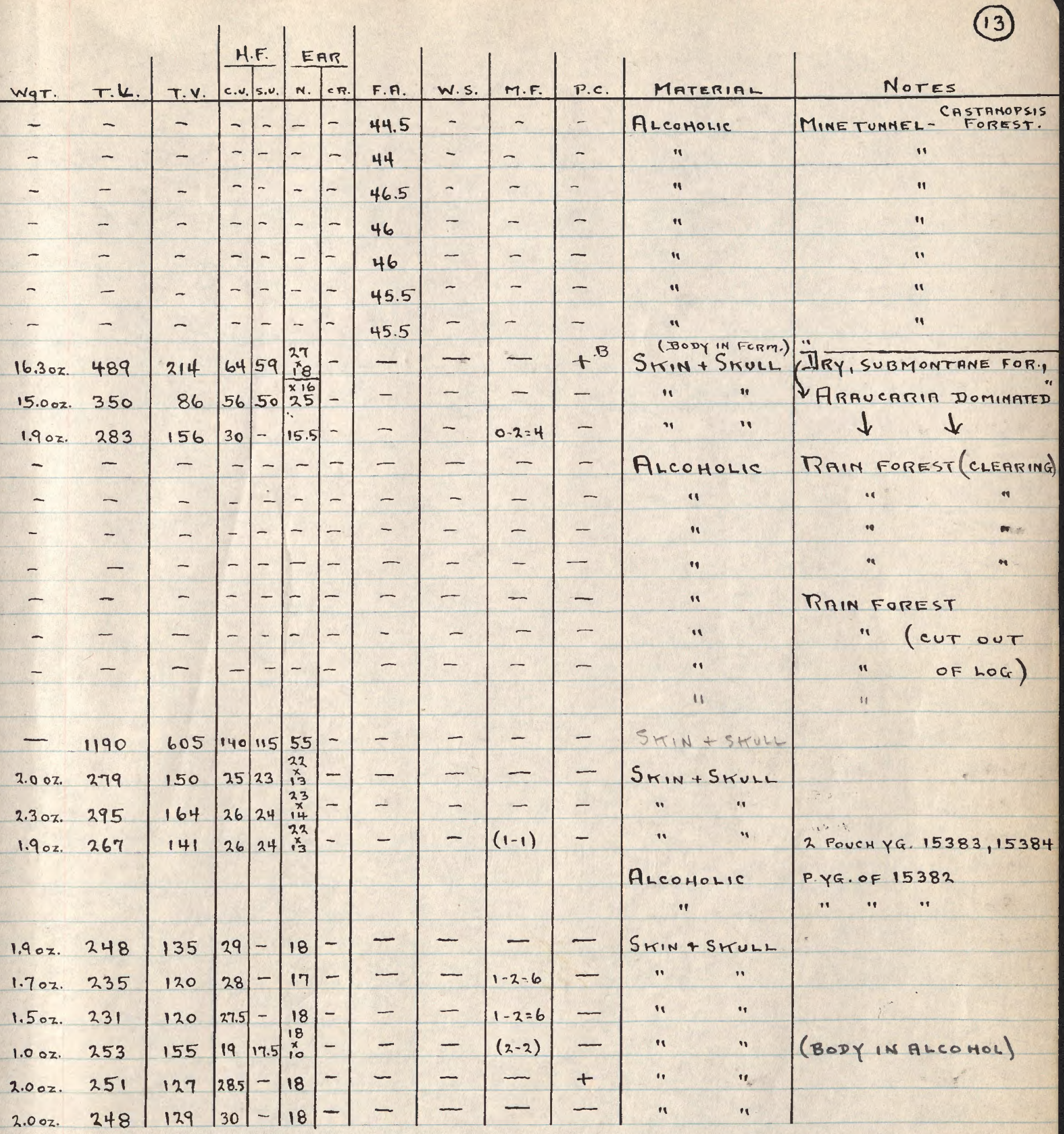


$+43 \ldots 1$

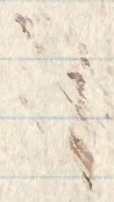



(14)

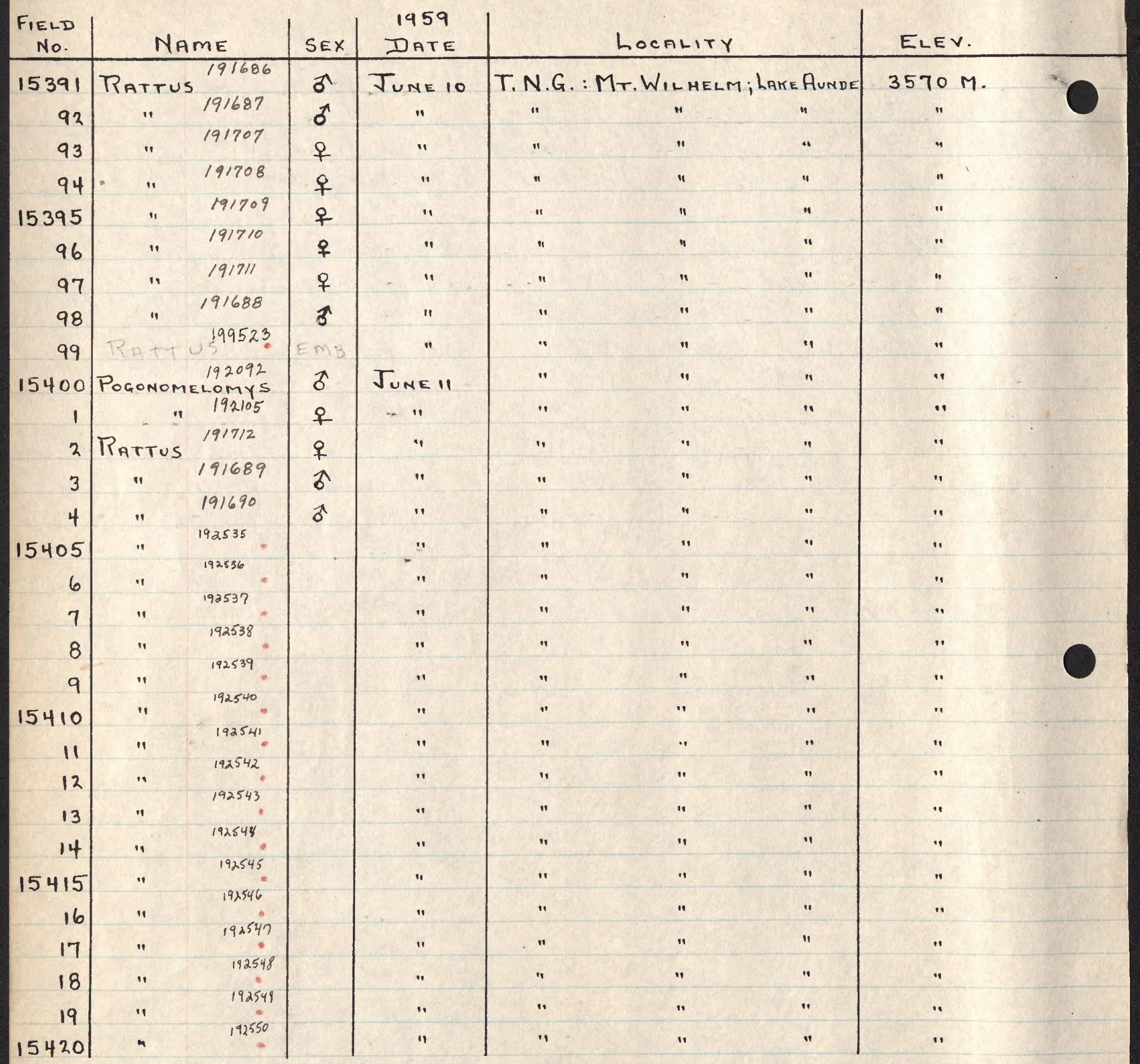




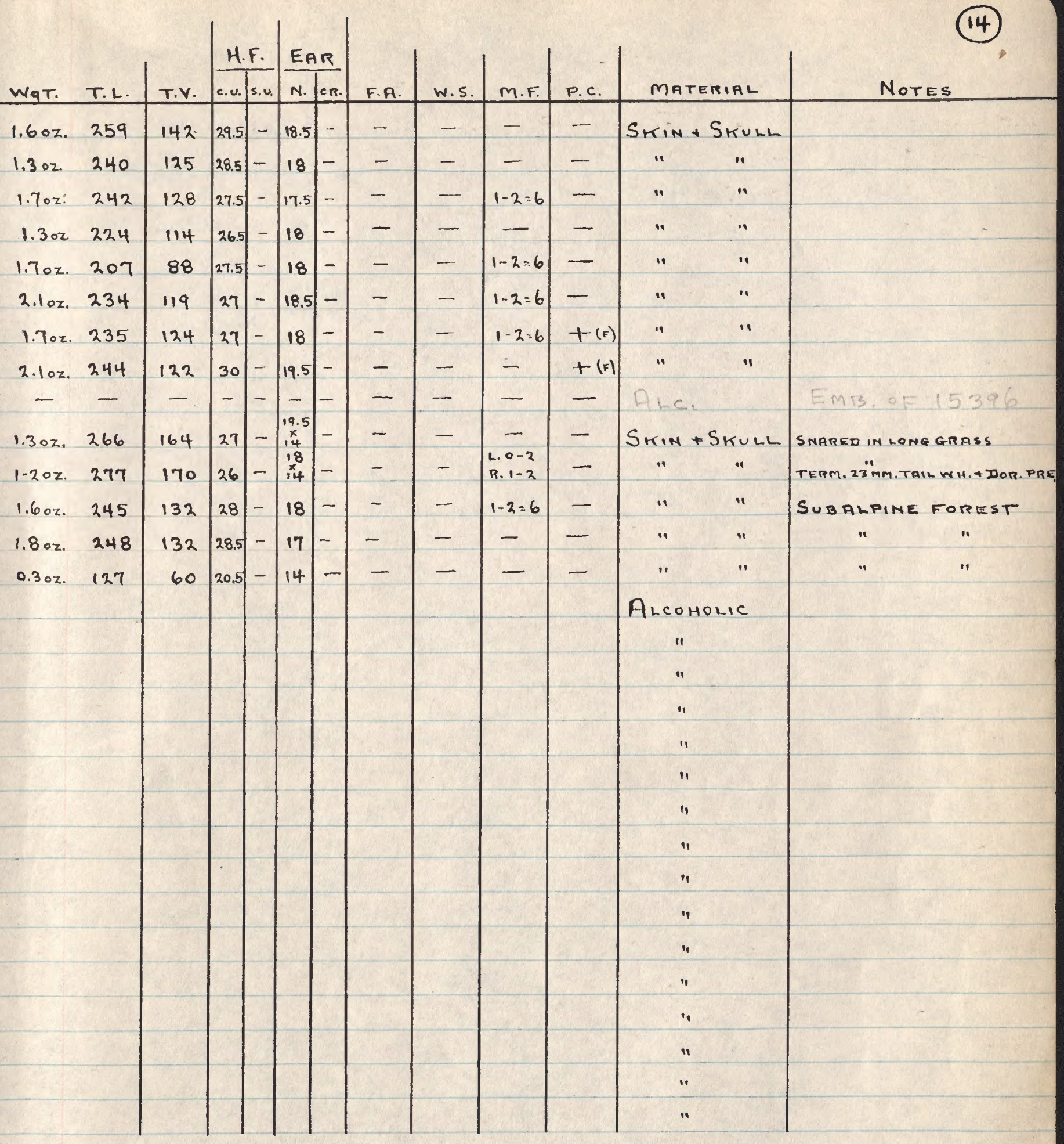






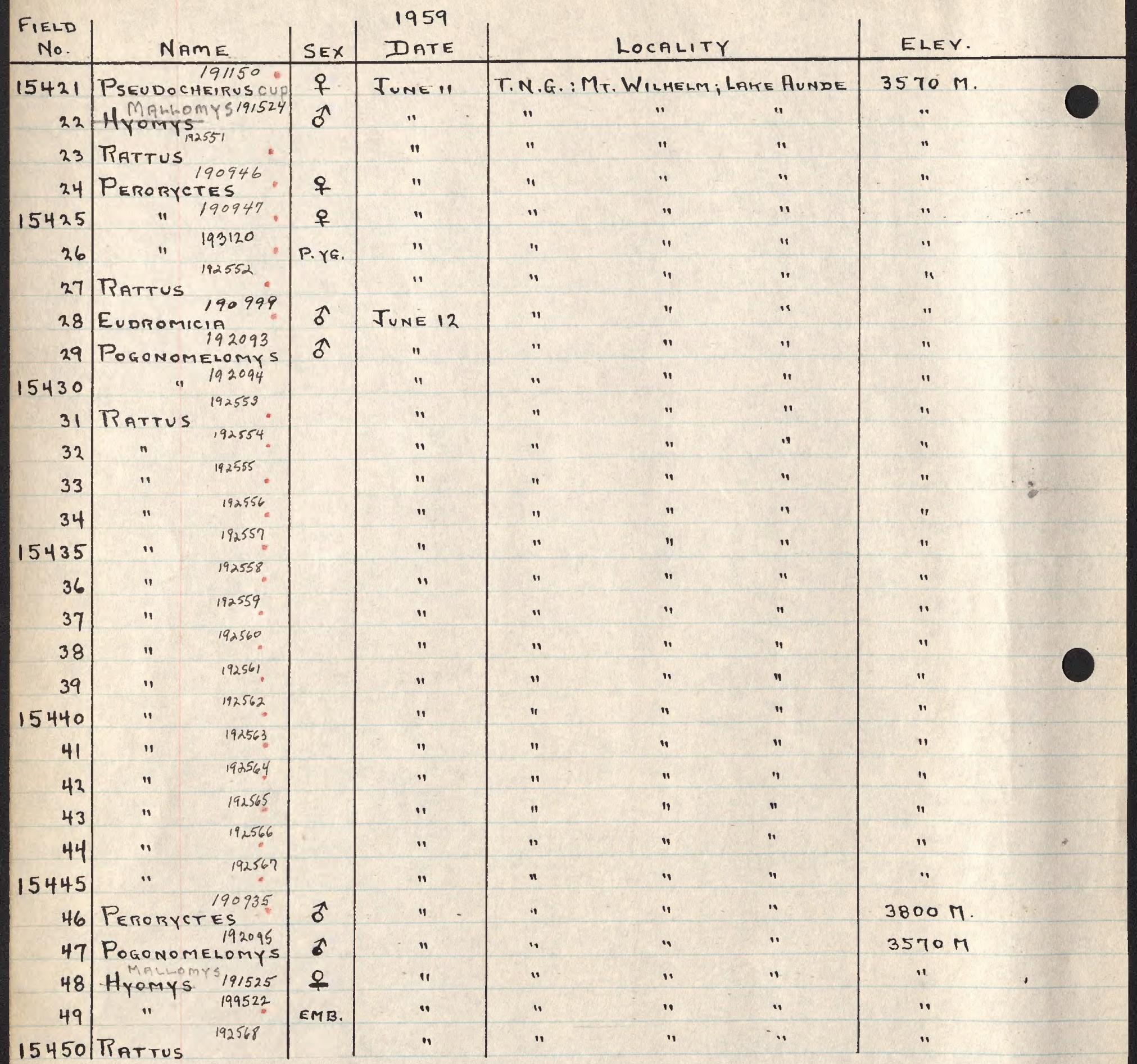




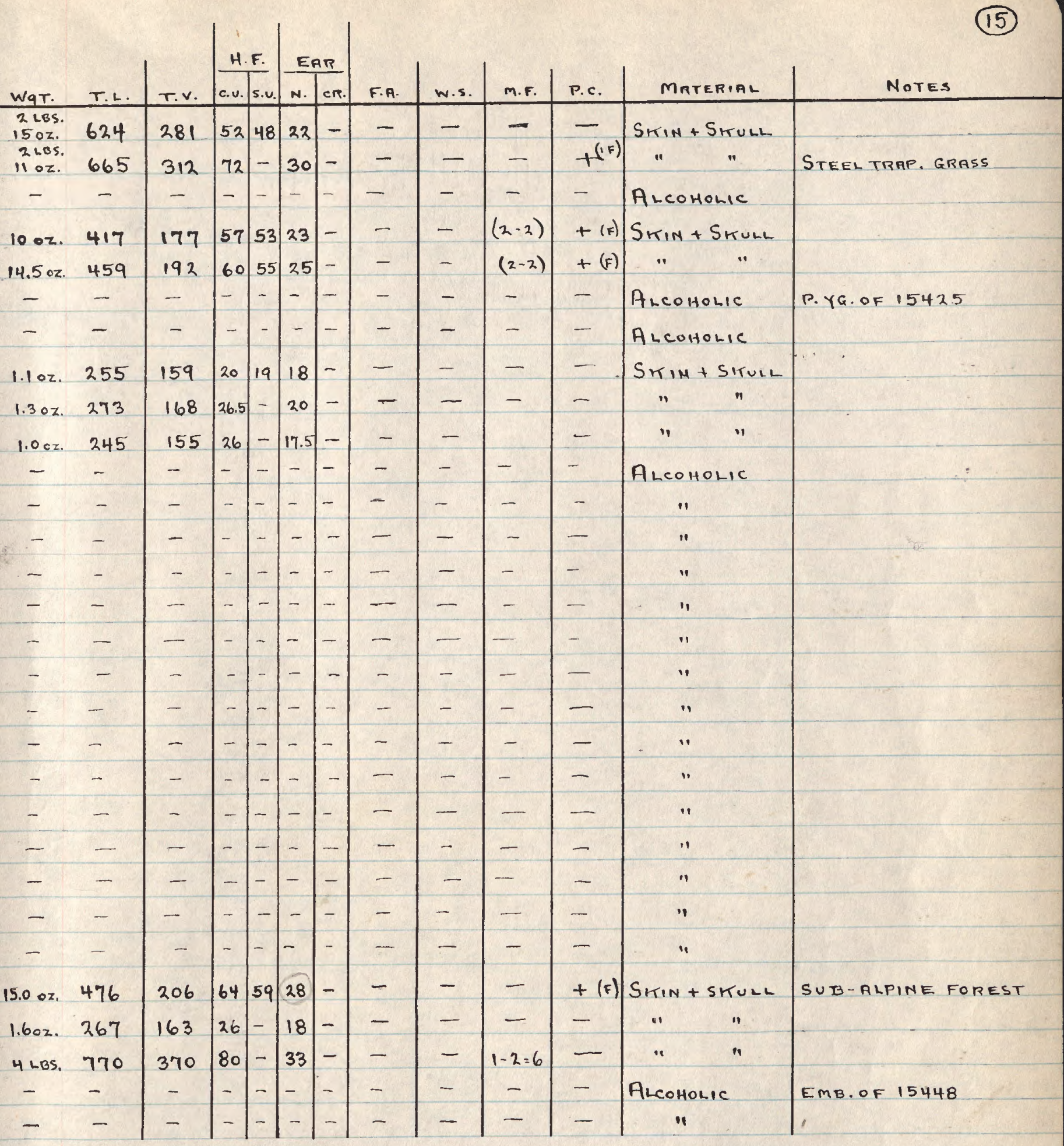

$15424,15425,15446$ : ECTOPARASITES FROM 3 SPECIMENS IN I VIAL. 




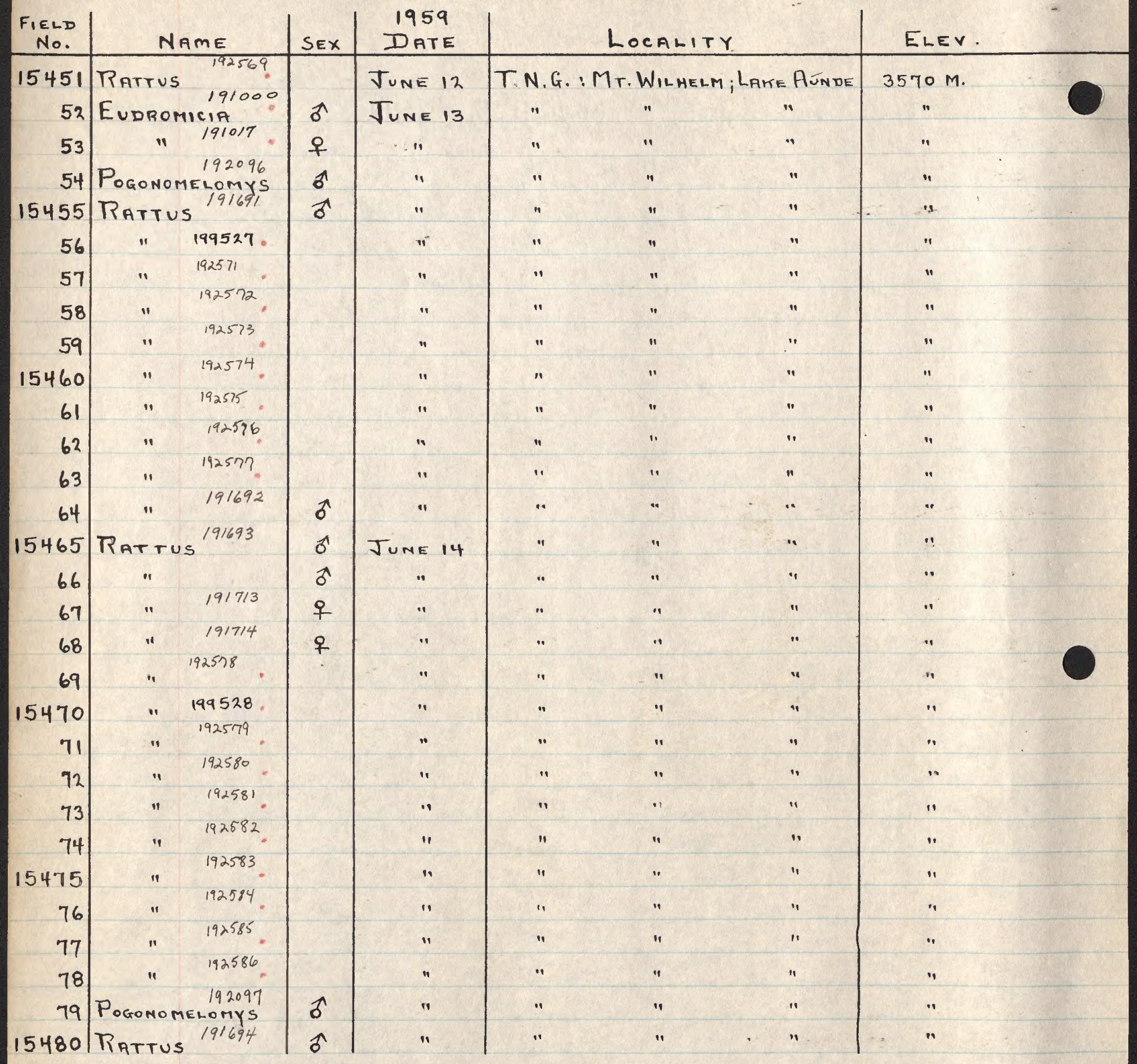






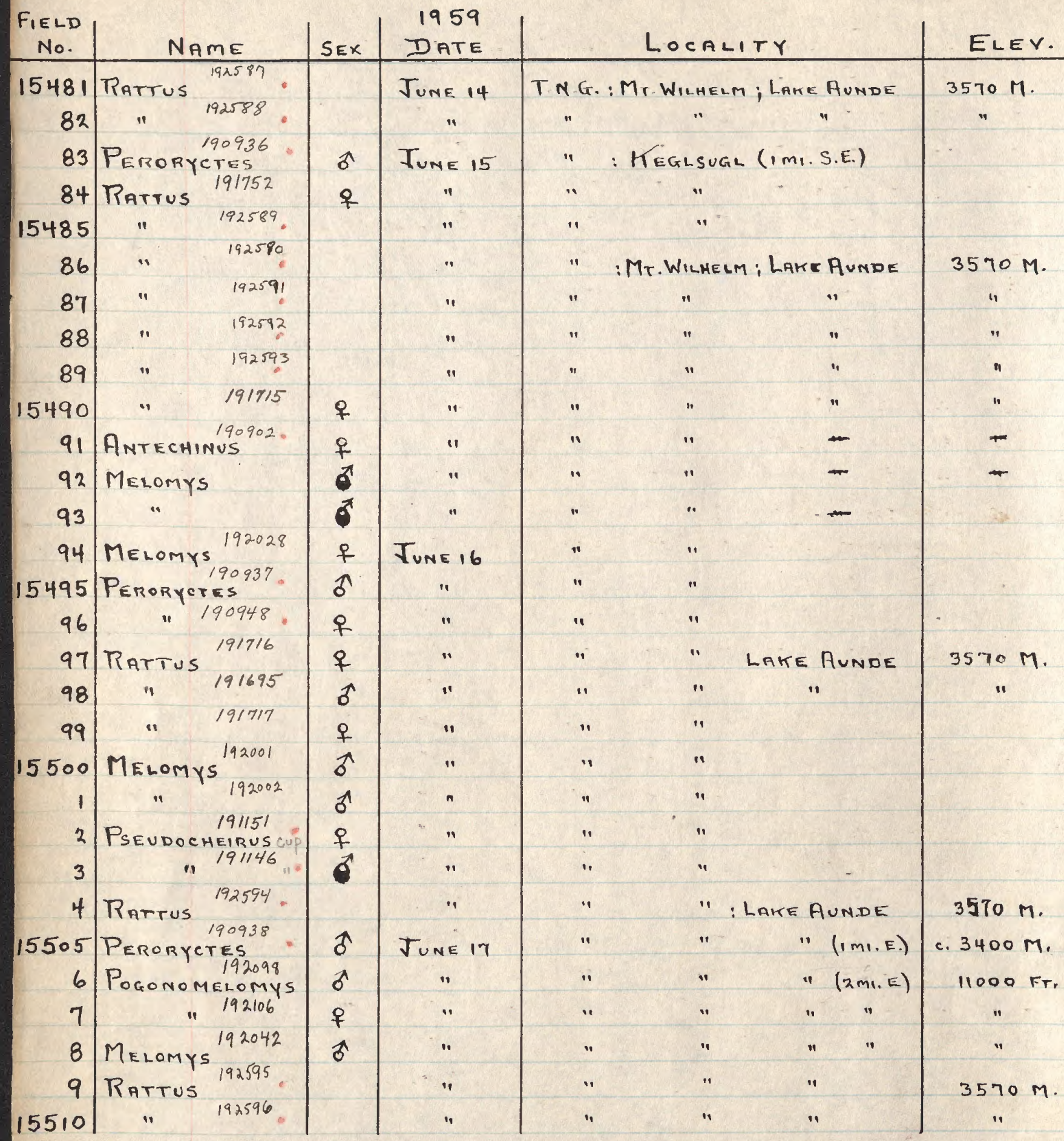





\section{0}

-

- 
-

-

- 


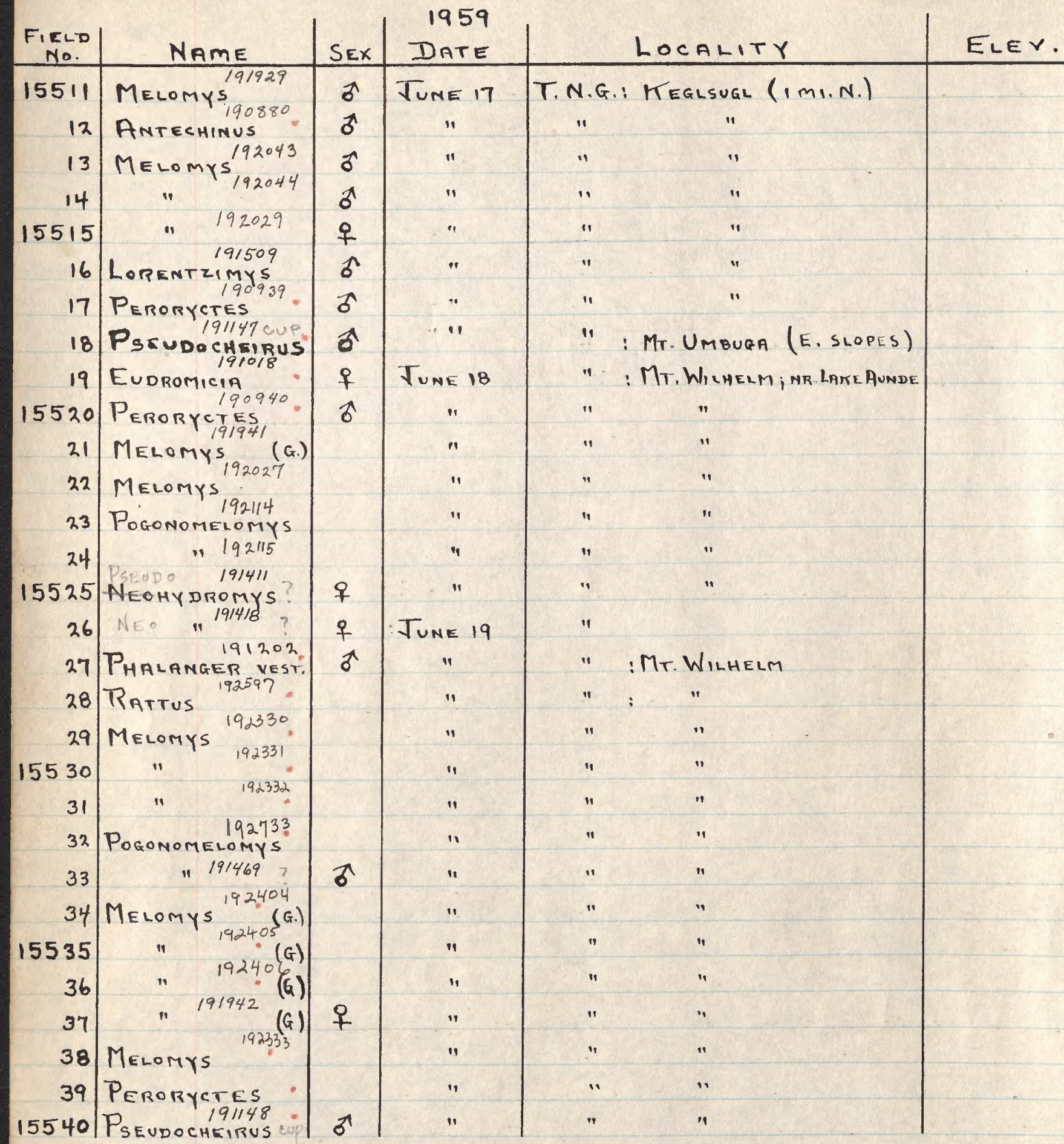




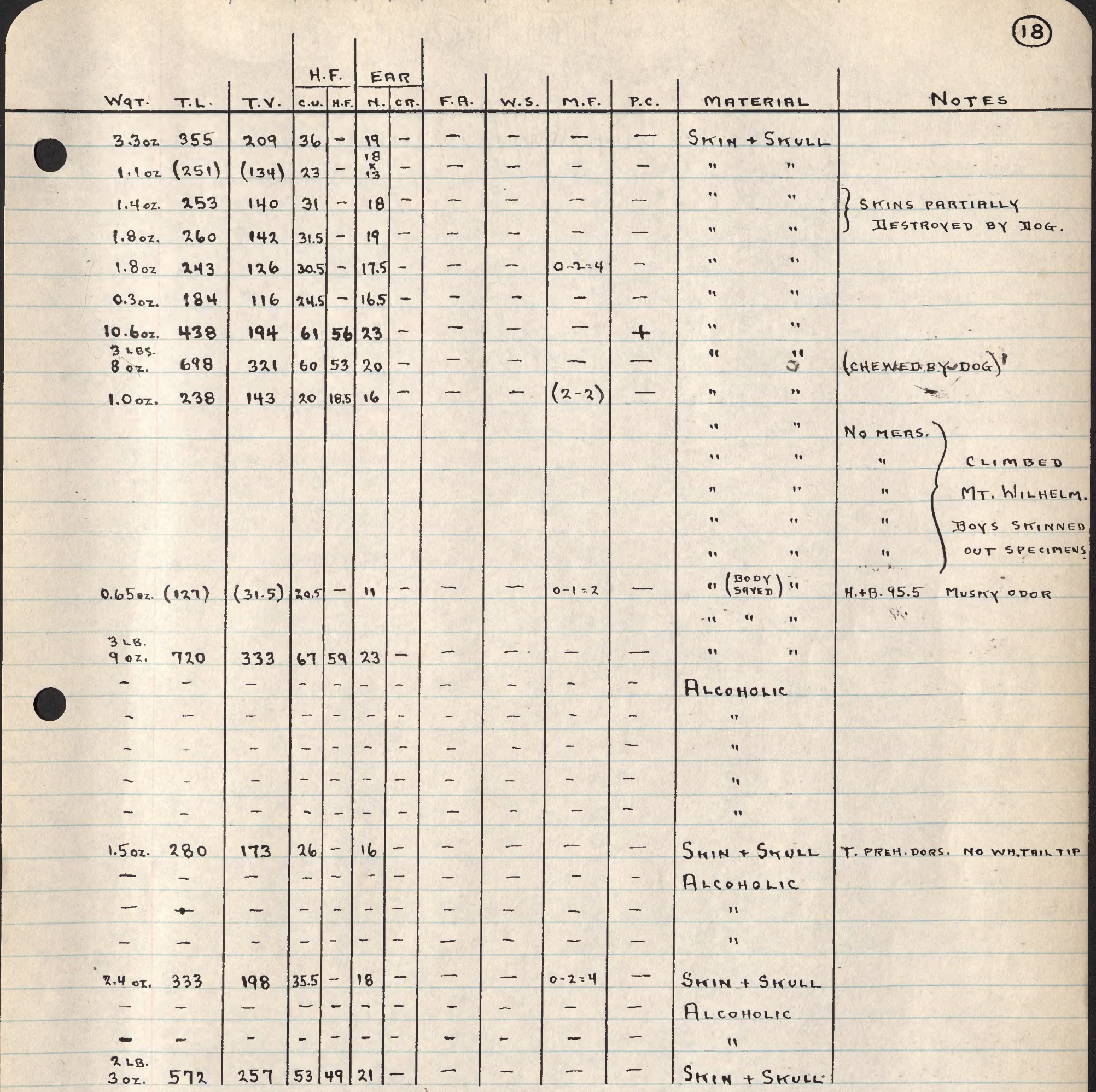

$15508 ; 15513 ; 15514$ SITINS CHEWED BY DOG (ALSO 15518)

$15520-24$ NO MEAS. (VD. CLIMBING MT. WILHELM) 



\begin{tabular}{|c|c|c|c|c|c|c|c|}
\hline $\begin{array}{c}\text { FIELD } \\
\text { NO. }\end{array}$ & NAME & SEX & $\begin{array}{l}1959 \\
\text { DATE }\end{array}$ & \multicolumn{3}{|c|}{ LOCALITY } & ELEV. \\
\hline 15541 & $\begin{array}{c}192157 \\
\text { PSEUdACHEIRUS CUP }\end{array}$ & $\hat{\sigma}$ & TUNE 19 & $T \cdot N \cdot G$ & & $=$ & \\
\hline 42 & "191152 & $q$ & " & 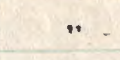 & " & & 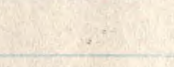 \\
\hline 43 & $\begin{array}{r}192107 \\
\text { PogONOMELOMYS }\end{array}$ & 오 & Tune 20 & $"$ & " & Lake Aunde & $3570 \mathrm{M}$. \\
\hline 44 & Rattus $1917 / 8$ & 오 & $"$ & $"$ & $"$ & $"$ & $"$ \\
\hline 15545 & " $192598^{\circ}$ & & $"$ & $"$ & $"$ & $"$ & " \\
\hline 46 & $\begin{array}{l}1192599 \\
192600^{\circ}\end{array}$ & & $"$ & $"$ & $"$ & $"$ & $"$ \\
\hline 47 & " 142600 . & & 11 & $"$ & " & $"$ & ." \\
\hline 48 & MELOMYS 192334 & & $"$ & $\checkmark$ & $"$ & & \\
\hline 49 & 1114233 & & $"$ & $"$ & $"$ & & \\
\hline 15550 & 192003 & $\delta$ & " & $"$ & $"$ & & \\
\hline 51 & " 192030 & 우 & $"$ & " & $"$ & & \\
\hline 52 & MELOMYS & $\widehat{\sigma}$ & " & $"$ & " & & \\
\hline 53 & PoGONOMYS (GR.) & f & " & " & " & & \\
\hline 54 & PERORYCTES & 우 & " & " & $"$ & & \\
\hline 15555 & " & $q$ & v & $"$ & $"$ & & \\
\hline 56 & $\begin{array}{c}191153 \text { CUP } \\
\text { PSEUDOCHEIRUS }\end{array}$ & 오 & $"$ & " & $"$ & & \\
\hline 57 & Peroryctes & $\hat{\delta}$ & $"$ & " & $"$ & & \\
\hline 58 & PoGONOMELOMYS & $\delta$ & $"$ & $"$ & $"$ & & \\
\hline 59 & MELOMYS & & $"$ & $"$ & $"$ & & \\
\hline 15560 & $\begin{array}{l}1192004 \\
192601\end{array}$ & $\hat{\sigma}$ & $"$ & " & $"$ & & \\
\hline 61 & Rattus $192602^{\circ}$ & & JunE 21 & $"$ & $"$ & & \\
\hline 62 & $\begin{array}{l}192602^{\circ} \\
119260^{\circ}\end{array}$ & & $"$ & $"$ & " & & \\
\hline 63 & 192603 & & " & " & $"$ & & \\
\hline 64 & 192604. & & " & " & $"$ & & \\
\hline 15565 & $\begin{array}{l}192731 \\
\text { NEOHYOROMYS }\end{array}$ & $f$ & " & $"$ & $"$ & & \\
\hline 66 & $\begin{array}{c}191510 \\
\text { LORENTZIMYS }\end{array}$ & $\hat{\sigma}$ & $" 1$ & $"$ & $"$ & & \\
\hline 67 & Pogomomys (GR) & & " & $"$ & $"$ & & \\
\hline 68 & " 192301" & & $"$ & $"$ & " & & \\
\hline 69 & 192302 & & $"$ & $"$ & " & & \\
\hline $15570 \mid$ & $"$ & & $"$ & $"$ & " & & \\
\hline
\end{tabular}




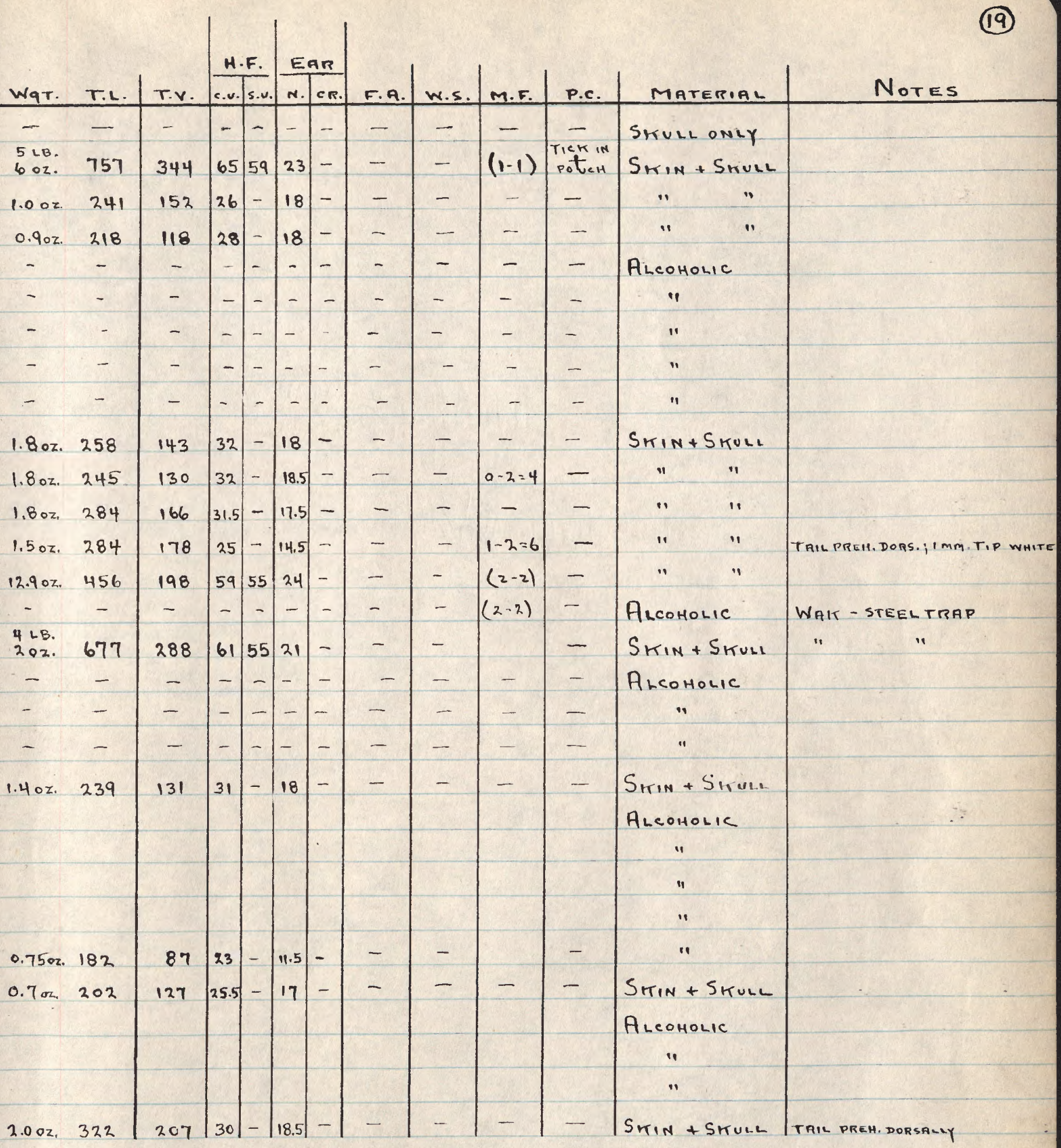



O

0

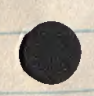


(20)

\begin{tabular}{|c|c|c|c|c|c|}
\hline $\begin{array}{c}\text { FIELD } \\
\text { No. }\end{array}$ & NAME & SEX & $\begin{array}{c}1959 \\
\text { DATE }\end{array}$ & LOCALITY & ELEV. \\
\hline 15571 & $\begin{array}{l}190903 \\
\text { Antechinus }\end{array}$ & q & TUNE 21 & T.N.G. : MT. WILHELM & \\
\hline 72 & " 192270 & & "1 & " n & \\
\hline 73 & "190881=. & $\hat{\sigma}$ & $"$ & $"$ & \\
\hline 74 & "1 192271 & न & $"$ & $"$ & \\
\hline 15575 & $\because \quad 192272$. & $q$ & $"$ & $"$ & \\
\hline 76 & PoGonomys 191480 & $q$ & $"$ & $"$ & \\
\hline 77 & MelomYS $19240 ?$ & & $"$ & $"$ & \\
\hline 78 & "192408 & & "1 & $"$ & \\
\hline 79 & 192409 & & " & " & \\
\hline 15580 & " 192410 & & $"$ & $"$ & \\
\hline 81 & " 192411. & & " & $"$ & \\
\hline 82 & MELOMYS $\begin{array}{c}192337 \\
(\text { GR })\end{array}$ & & " & $"$ & \\
\hline 83 & $\begin{array}{r}192338 \\
192339\end{array}$ & & " & $"$ & \\
\hline 84 & $\begin{aligned} 192339 \\
11\end{aligned}$ & & " & $"$ & \\
\hline 15585 & 192340 & & $"$ & " & \\
\hline 86 & 192341 & & $"$ & $"$ & \\
\hline 87 & " $\quad 192342$ & & $"$ & $"$ & \\
\hline 88 & PogONOMELOMYS & & $"$ & $"$ & \\
\hline 89 & $\because \quad \begin{array}{ll}192736 \\
\quad 192737\end{array}$ & & $"$ & " & \\
\hline 15590 & " 192737 & & $"$ & $"$ & \\
\hline 91 & 14 $\quad 192099$ & $\hat{\sigma}$ & $"$ & " & \\
\hline 92 & $\begin{array}{c}190904 \\
\text { AntECHINUS } \\
\text { 191931 }\end{array}$ & q & TUNE 22 & " & \\
\hline 93 & MELOMYS (BR) & 3 & $"$ & $"$ & \\
\hline 94 & MELOMYS ${ }^{192005}(G x)$ & ๙ & $"$ & $"$ & \\
\hline 15595 & 192006 & 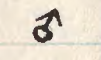 & 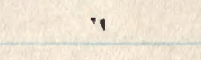 & " & \\
\hline 96 & 192007 & 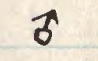 & $"$ & $"$ & \\
\hline 97 & $192343=$ & & $"$ & " & \\
\hline 98 & 192344. & & $"$ & $"$ & \\
\hline 99 & $\begin{array}{l}142345 \\
192346\end{array}$ & & $"$ & $"$ & \\
\hline 15600 & $"$ & & " & $"$ & \\
\hline
\end{tabular}




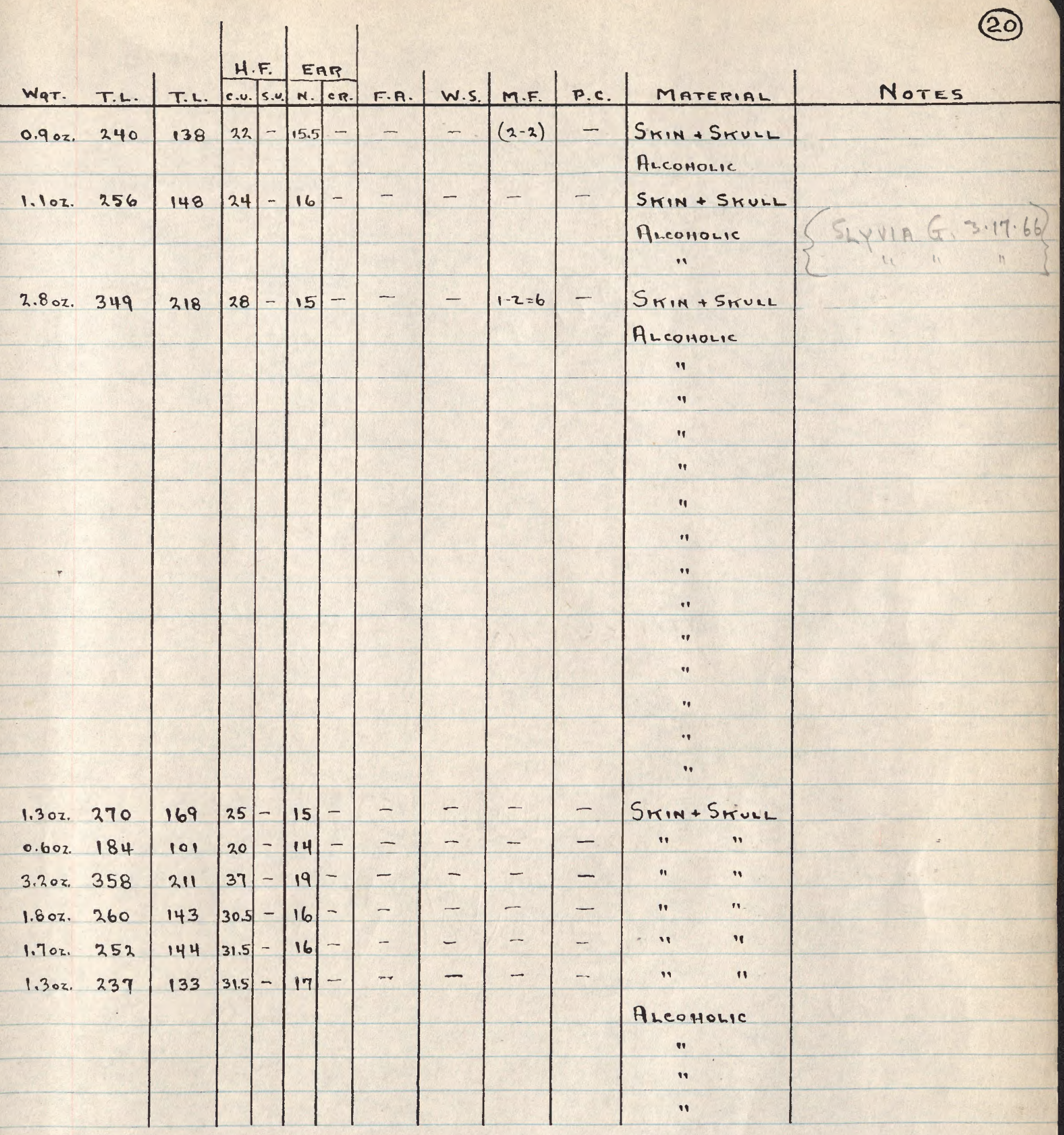



0

-

0 


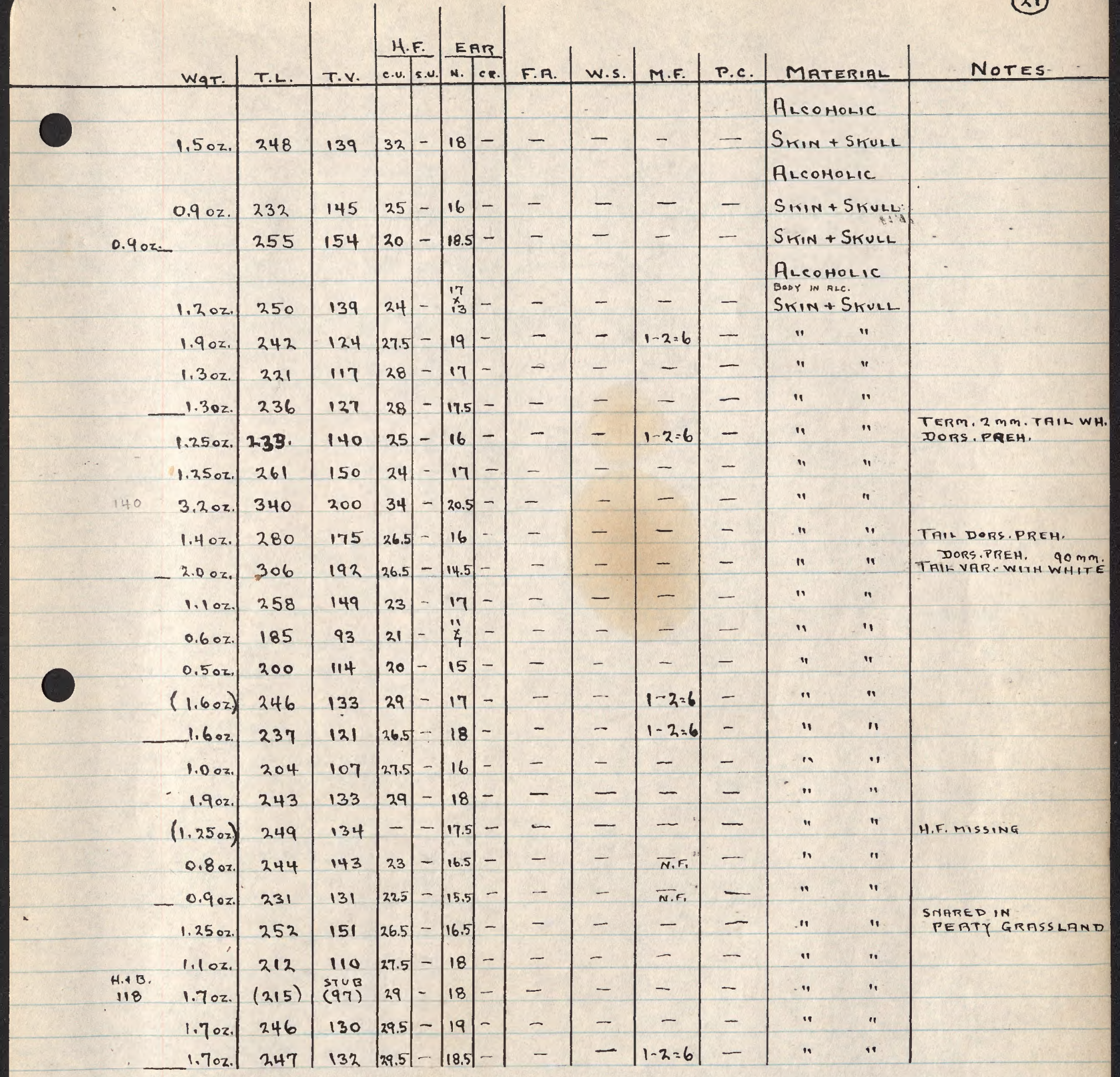



O

-

0 


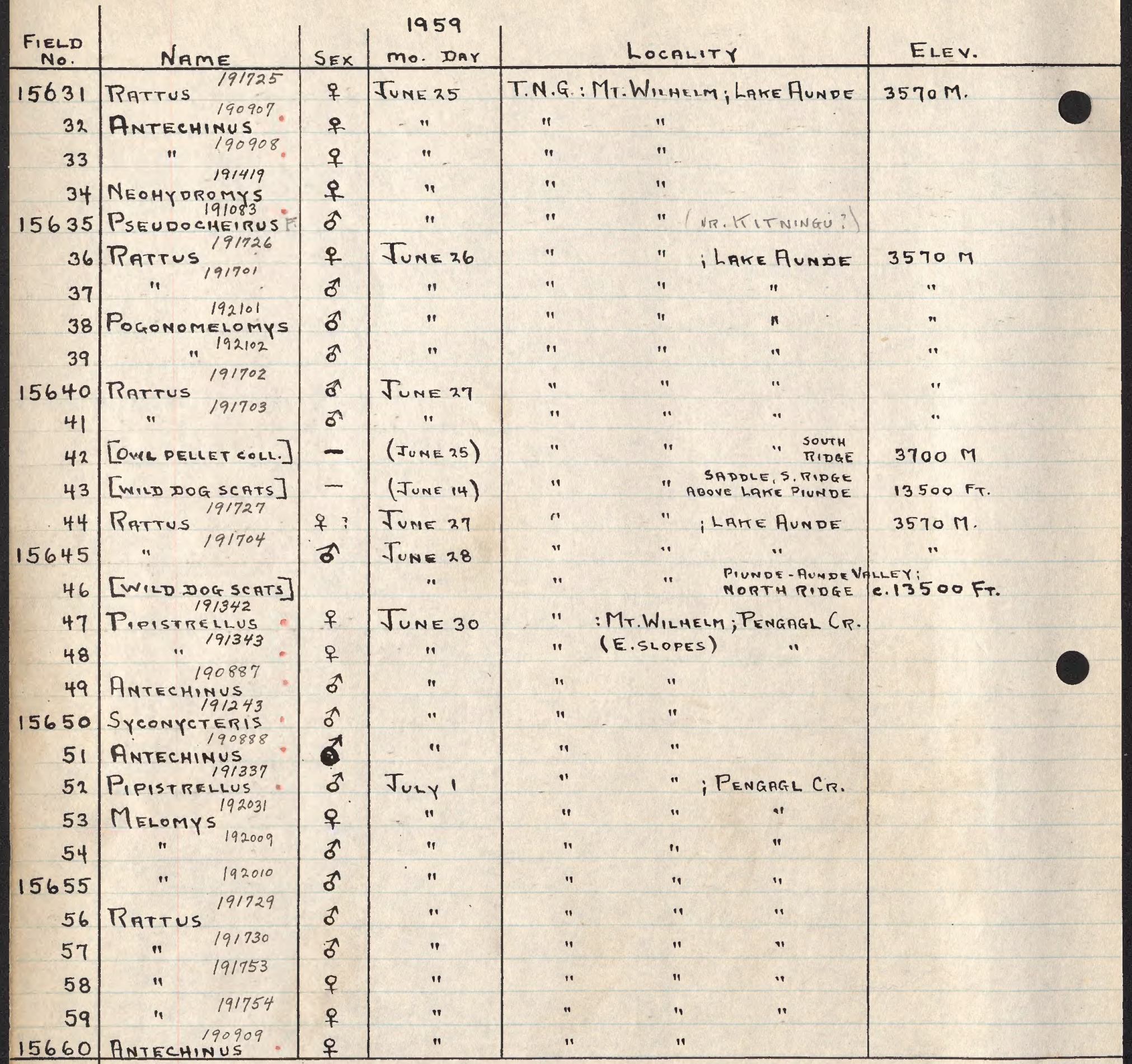

Parasites: Bird" \#13 Mt.Wilhelm" Brought in With \#s 15640,15641 . I COLLECTED FLEFS + MITES FROM THIS BIRD. HOWEVER, THEY MAY HAYECOME FROM R. NIODE. CAN EASILY DE CHECKED:

1) CCLLECTED MITES FROM 15640, 15641 (IN I VIAL)
2) " FLERS + MITES FTOM 15645 . (R. NIOBE) 
H.F. EAT WGT. T.L. T.V. C.U. S.U. N. CR.

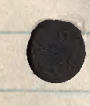

\begin{tabular}{|c|c|c|c|c|c|c|c|c|c|c|c|c|c|}
\hline & WaT. & T.L. & T.V. & c.u. & s.u. & N. & $\overrightarrow{C R}$. & F.A. & W.S. & m.r. & P.. . & Material & Notes \\
\hline & $2.0 \mathrm{oz}$ & .238 & 126 & 28 & - & 19 & - & - & - & $1-2=6$ & - & Srin + Srruel & \\
\hline & $1.00 z$ & 245 & 141 & 22.5 & - & 15.5 & - & 一 & - & $(z-z)$ & - & " & . \\
\hline & 0.602 & 197 & 112 & 20 & - & 14.5 & - & - & - & N.F. & - & $"$ & $\cdots$ \\
\hline $\begin{array}{c}H+B \\
95\end{array}$ & $0.90 z$ & 173 & 78 & 22 & - & 11.5 & - & - & - & $0-2=4$ & - & $"=$ & $\begin{array}{l}\text { EROWISH WASH BASEOF } \\
\text { TAIL,HHW OLES. }\end{array}$ \\
\hline & $\begin{array}{ll}2 & L B 5 \\
0 & 02\end{array}$ & 656 & 343 & 50 & 46 & 20 & - & - & - & - & - & $\therefore$ & \\
\hline 114 & $(1.702)$. & $(228)$ & $(114)$ & 27 & $=$ & 16 & - & - & - & $1-2=6$ & 一 & $"$ & SMARE IN GRHSS \\
\hline 114 & $1.75 \cdot z$ & 238 & 124 & 27.5 & - & 17 & - & - & - & - & - & $"$ & SUBALPINE FOREST \\
\hline 96 & $1.20 z$ & 259 & 163 & 26 & - & 18 & - & - & - & - & - & $"$ & 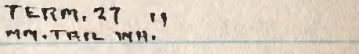 \\
\hline 93 & $1.00 z$ & 252 & 159 & 26 & - & 17.5 & - & - & - & - & $+(F)$ & $"$ & $\begin{array}{l}\text { DisTALL10 } \\
\text { mM. TAPL WN," }\end{array}$ \\
\hline 118 & $2.00 \%$ & $(234)$ & $(116)$ & 30 & - & 18.5 & - & - & - & - & $+?^{m}$ & $"$ & SUB-GLPINE FOREST \\
\hline 113 & $1.9 \circ 8$. & 234 & 121 & 29 & - & 18 & - & - & - & - & +5 & $"$ & " \\
\hline - & - & - & - & - & - & - & - & - & - & - & - & $\begin{array}{l}\text { ODD BOMES } \\
+ \text { PELLETS. }\end{array}$ & - Peaty "Grassland \\
\hline - & - & - & - & - & - & - & - & - & - & - & - & $\begin{array}{l}\text { CLAWES J IN SCRTS } \\
\text { DOMES }\end{array}$ & CREST BETWEEN VALLET \\
\hline & $0.70 x_{1}$ & 173 & 90 & 25,5 & - & 17 & - & - & - & - & - & Srrim + Skuke & \\
\hline & $1.5 \mathrm{oz}$ & 241 & 129 & 27 & - & 17 & - & 一 & 一 & 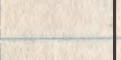 & $+p_{s}$ & "STELETON & SUB RLPINE FOREST \\
\hline - & - & - & - & - & - & - & - & - & - & - & - & 2 SCATS & ROCK + SHORT GRASS \\
\hline & $0.20 z$. & 93 & 42 & 9.5 & - & 12 & - & $(38)$ & $(282)$ & - & $\longrightarrow$ & Srrin + Sruex & OVER CREKK BED \\
\hline & $0.20 z$ & 92 & 40 & 9 & - & 12 & - & 38.5 & 287 & - & $t$ & " & " \\
\hline 82 & $0.60 z$ & 200 & 118 & 21 & - & 16 & - & - & - & - & - & $"$ & \\
\hline & $0.5 \mathrm{oz}$ & 75 & 一 & 13 & - & 13.5 & - & 42 & 329 & - & - & $"$ & \\
\hline & $1.30 x$ & 249 & 134 & 23.5 & - & 17 & - & - & 一 & - & 一 & $"$ & 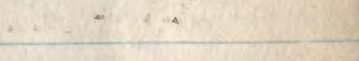 \\
\hline & $0.20 z$. & 88 & 38.5 & 9 & - & 12.5 & - & 37.5 & 272 & - & - & $"$ & OVER CREEK BED \\
\hline & $1.80 z$ & 242 & 126 & 31.5 & - & 18.5 & - & - & - & $0-2=4$ & + & $"$ & FOREST \{ \\
\hline & $1.80 z$ & 245 & 132 & 31 & - & 17.5 & - & - & - & - & - & " & " \\
\hline & $1.90 z$ & $(252)$ & 135 & 31 & - & 18 & - & - & - & - & - & " & •" \\
\hline & $1.7 a z$ & 242 & 132 & 30 & - & 17 & - & - & - & - & - & $"$ & " \\
\hline & 1.6ax. & 238 & 127 & 28.5 & - & 17.5 & - & - & - & 一 & 一 & $"$ & " \\
\hline & 1.502 & 252 & 141 & 30.5 & - & 19 & - & - & - & $1-2=6$ & - & $"$ & $"$ \\
\hline & 1.20z. & 212 & 112 & 28 & - & 18 & - & - & - & - & - & $"$ & $"$ \\
\hline & $0.90 z$ & 235 & 131 & 21 & -1 & 16 & - & - & - & $|(2-2)|$ & - & $"$ & 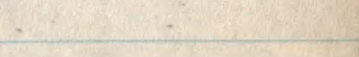 \\
\hline
\end{tabular}






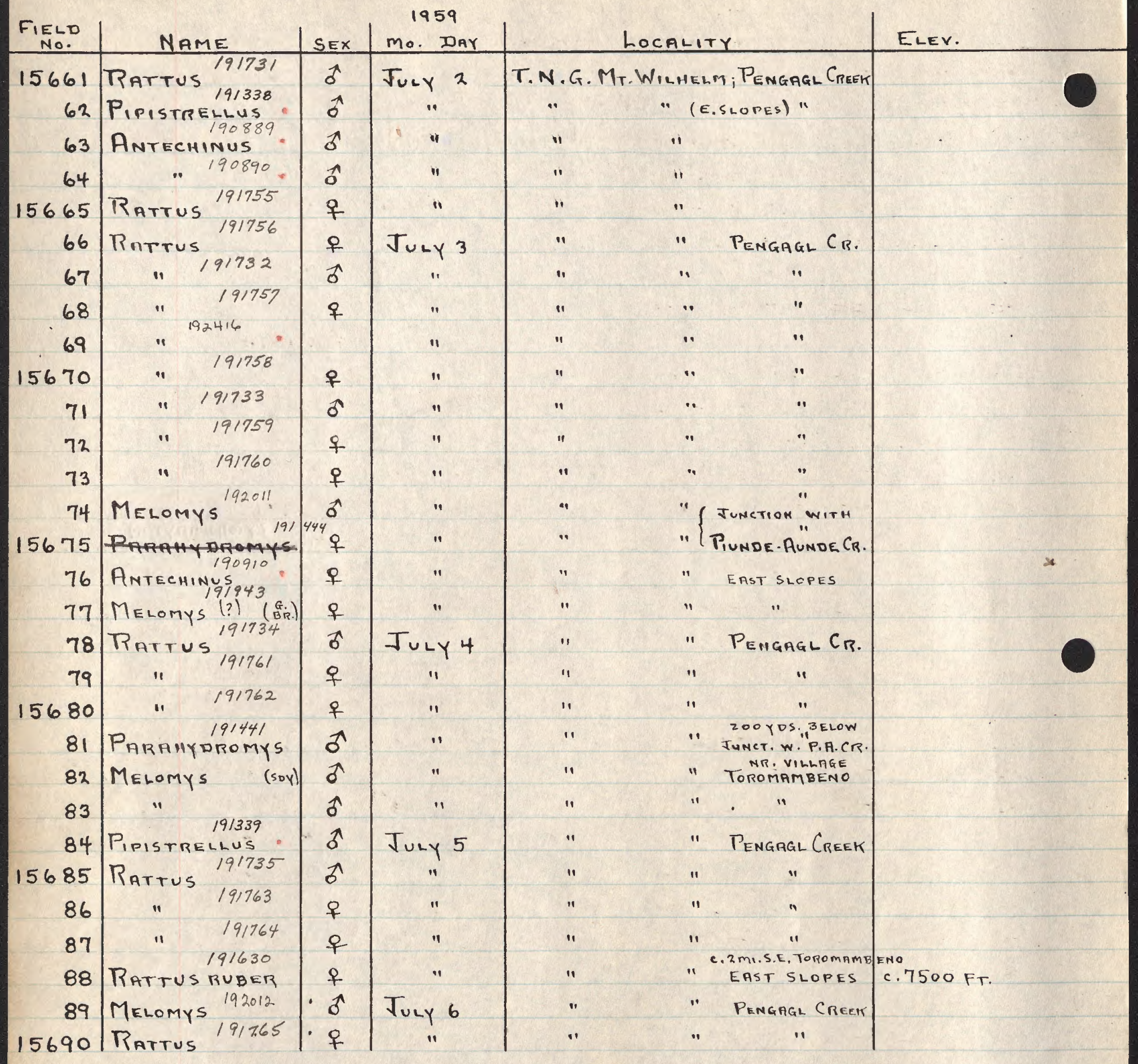


-

•

- 


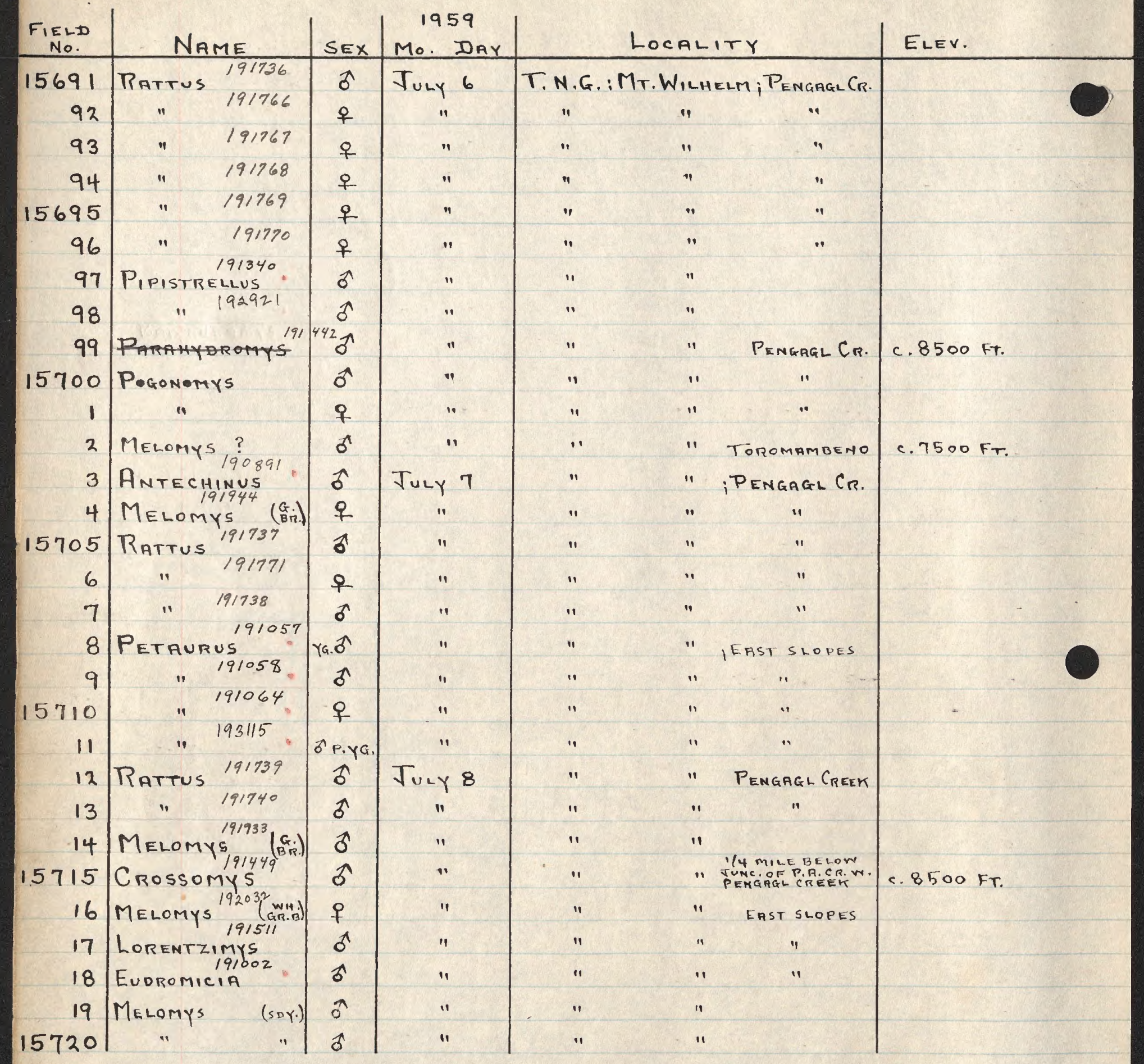

15715 TRAPPED \# O SINGLE SPRING STEEL, SIDE OF POOL $10 \times 15$ FT.1 UNDER LARGE DOULDER, NARROW LEDGE AT WATER LEVEL, TRAP PARTLY IN WATER, MUSEUM BAIT, CAUGHT BY I FRONT FOOT, TREES + SHRUBS TO EDGE OF STREAM, CURRENT THRU CENTEB OF POOL, POOL 2-3' DEEP, SAND + SMALL STONES ON DOTTOM, STILL. A MT. BROOK 


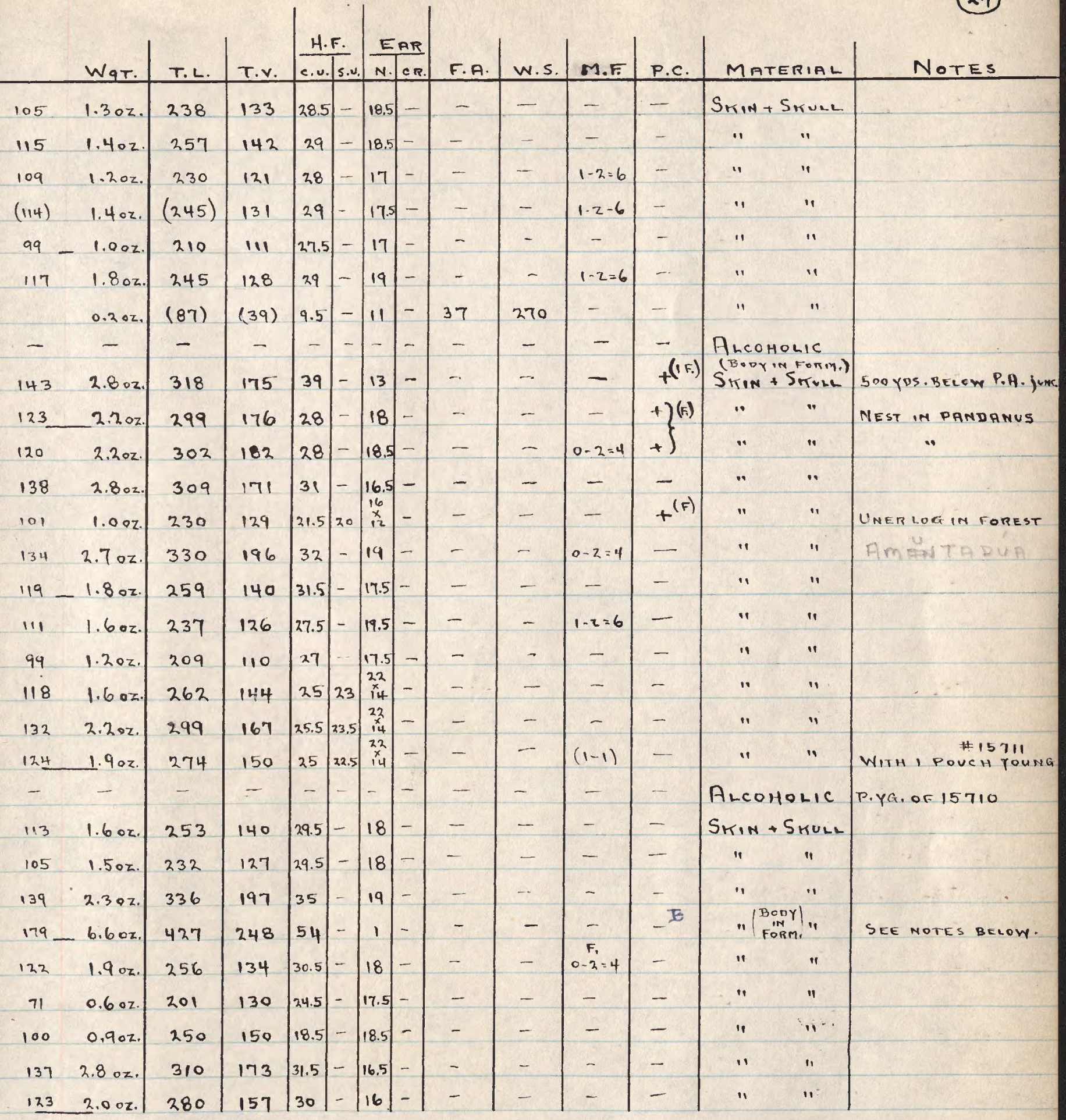



-

-

- 


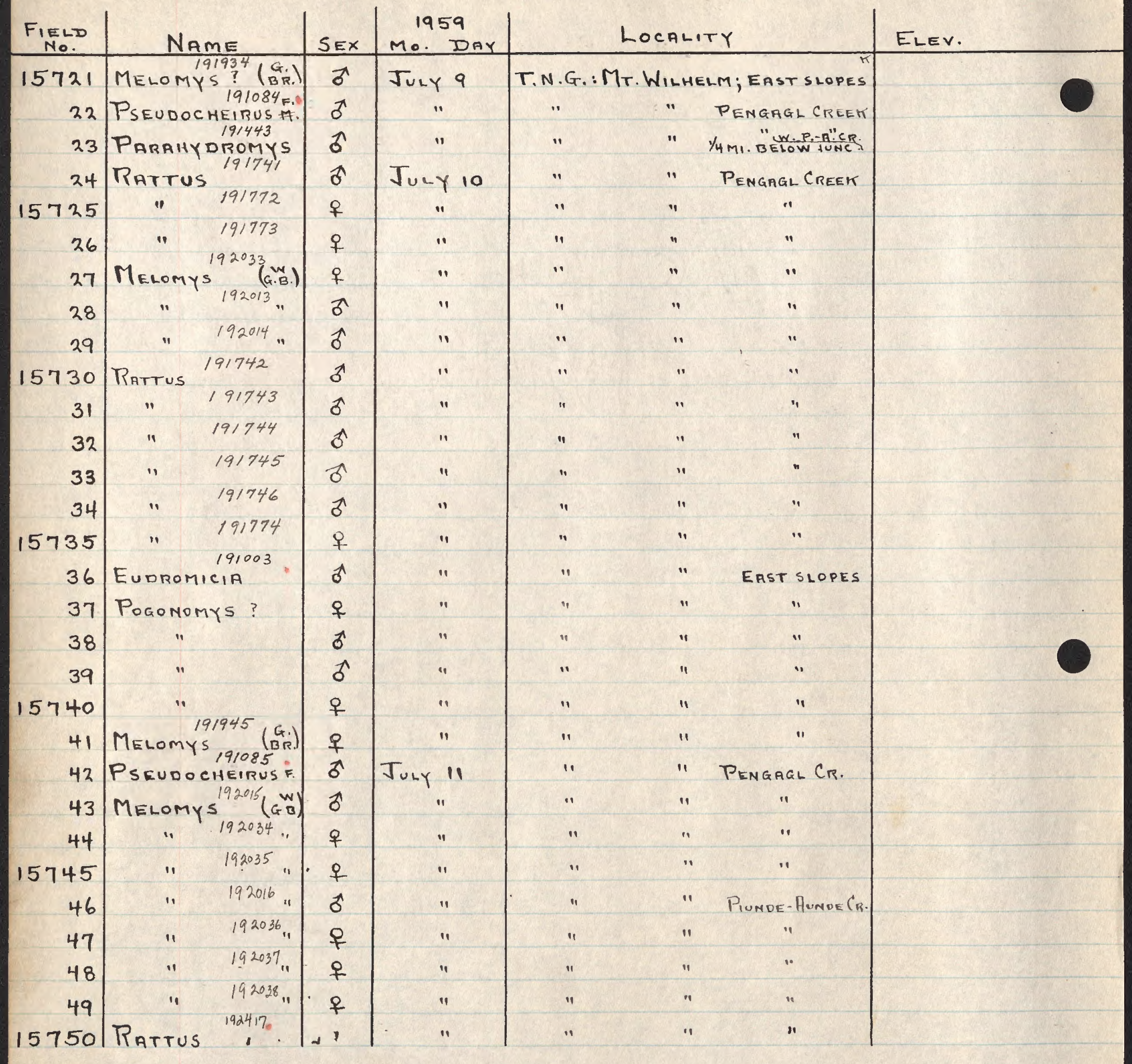




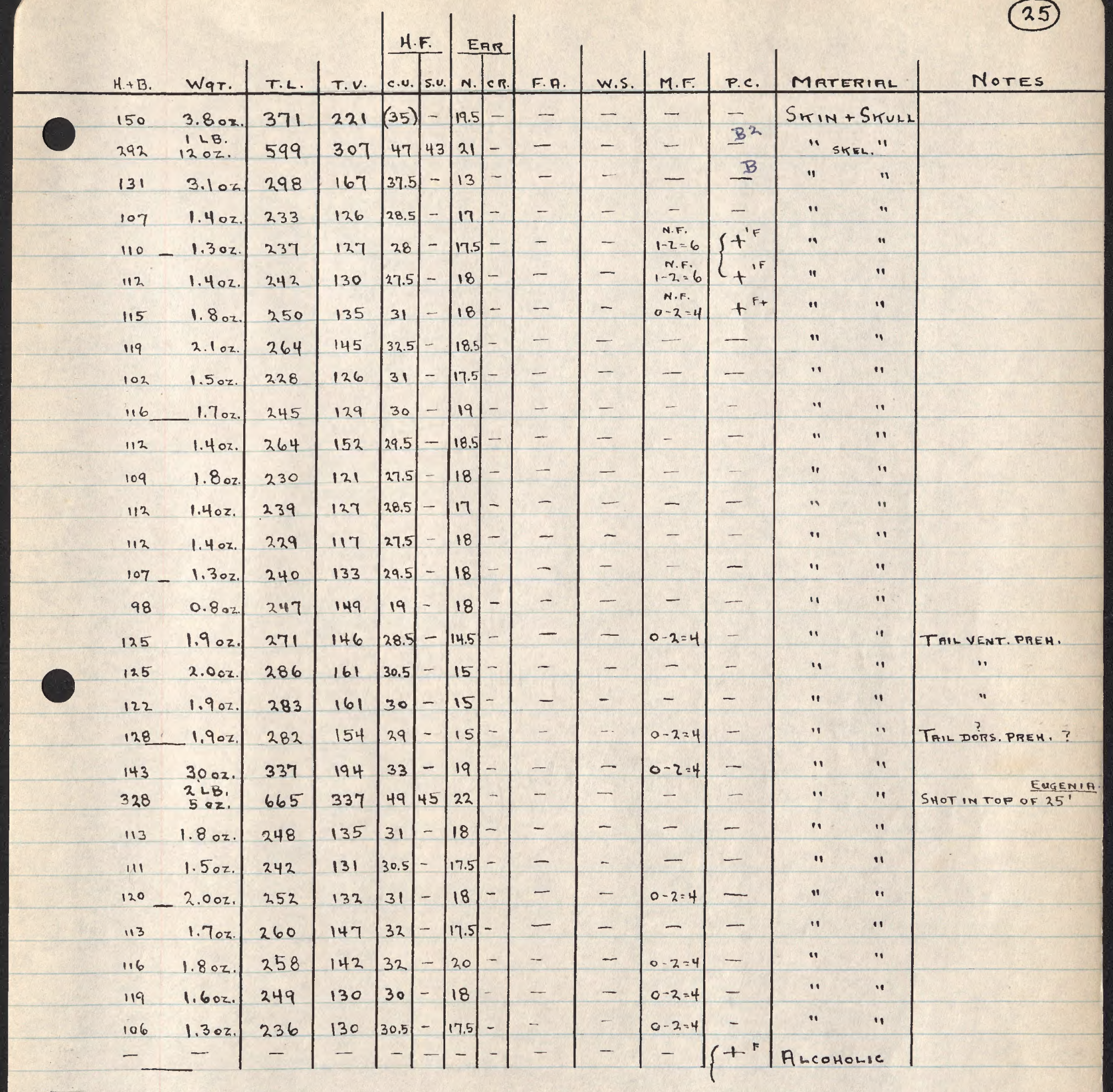



0

-

- 



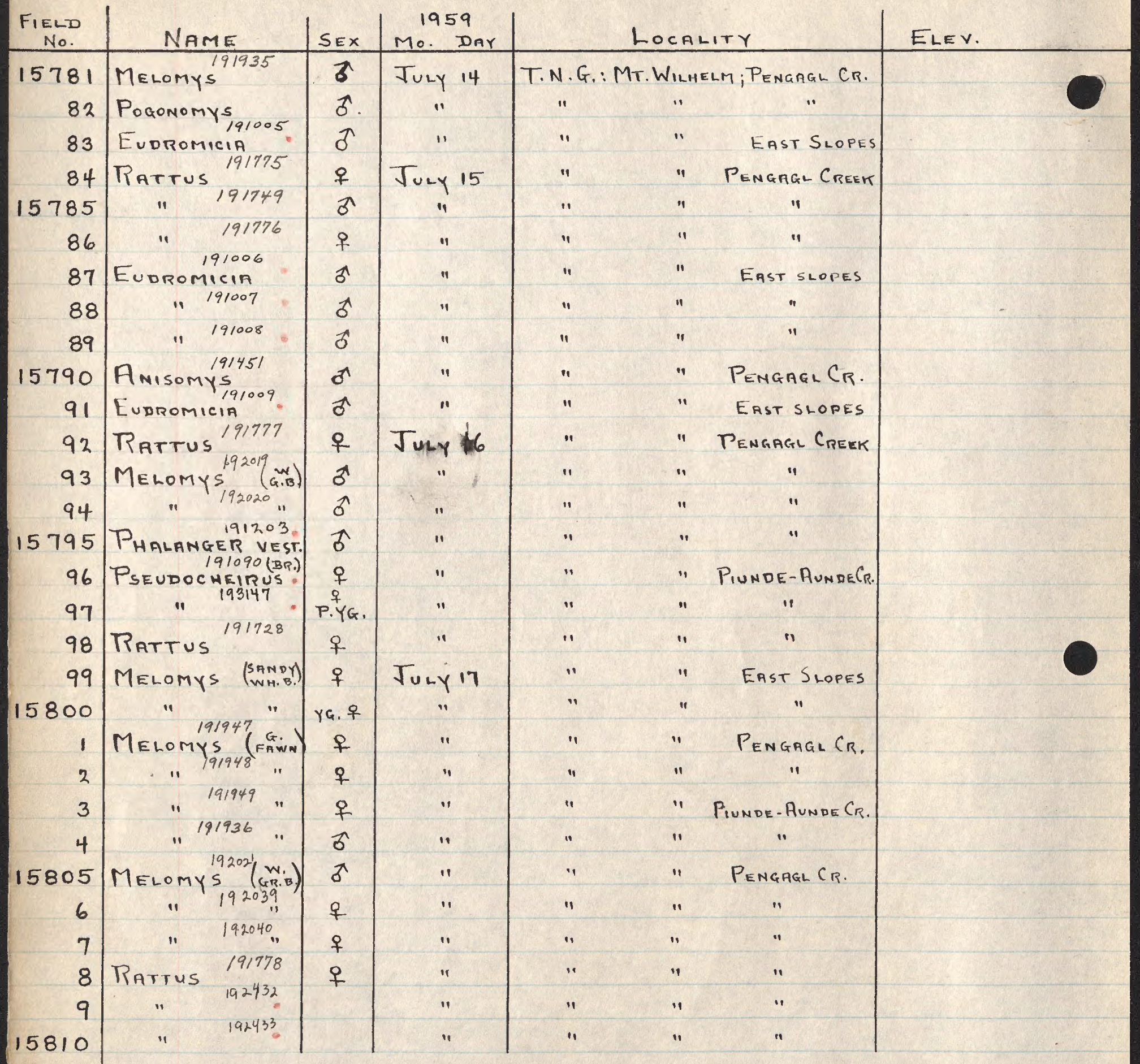




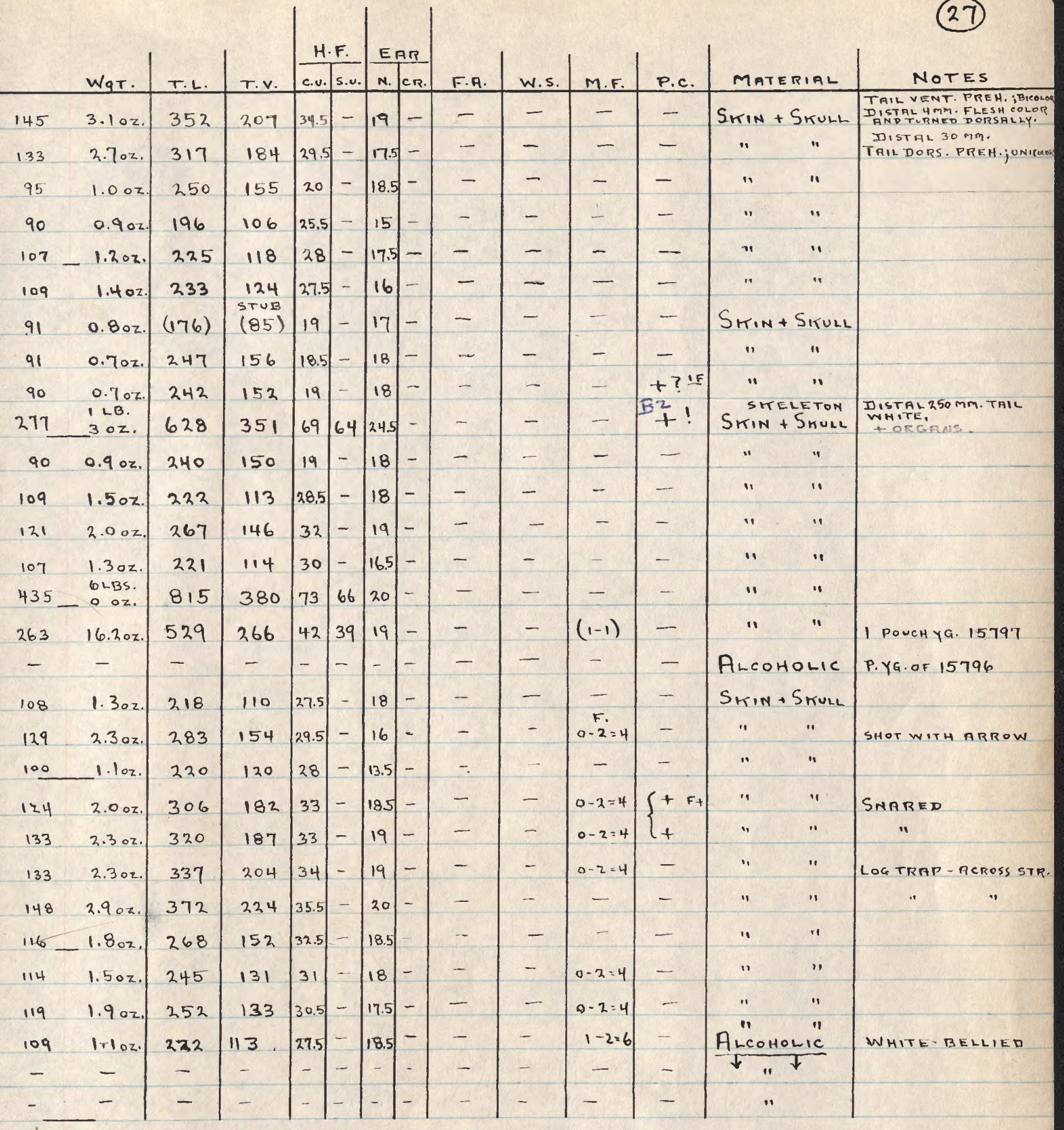

\footnotetext{
15781 - TAIL HAS DARK MID-VENTRAL STREAK
}

15789 - THERE IS SOME DOUBT AS TO WHETHER OR NOT THIS FLEA BELONGS TO EUDROMICIA. IT WASTAKENFROMTHIS SPECIMEN; HOWEVER, TIRTTUS NIOBE RND ANISOMYS WERE ALSO ON THETABLE. FLEAS FROM BOTH OF THESE SPECIES WERE CCLLECTED, SO IT MAY BE PASSIBLE TO CROSS CHECK. 
0

-

$\bullet$ 


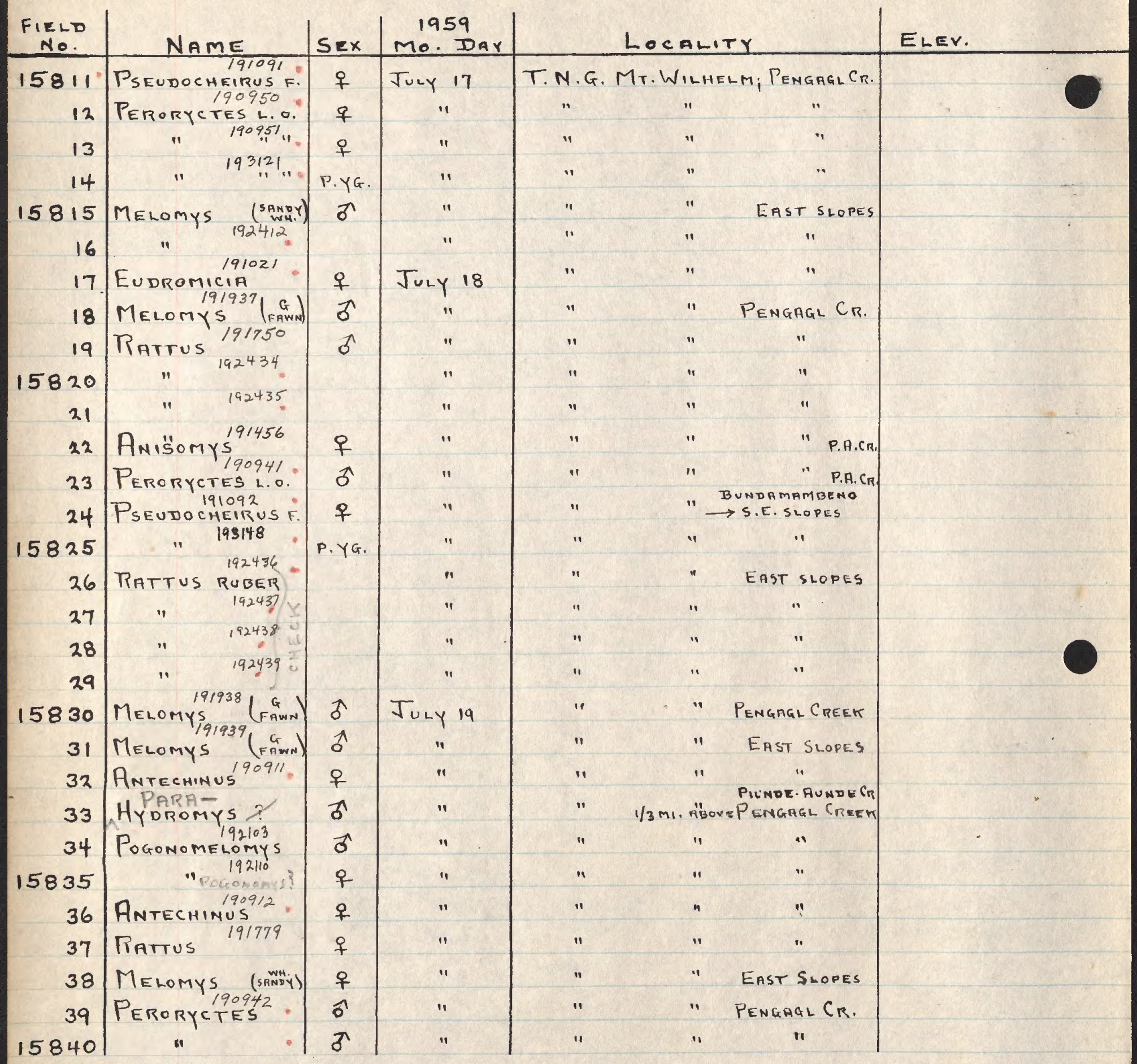




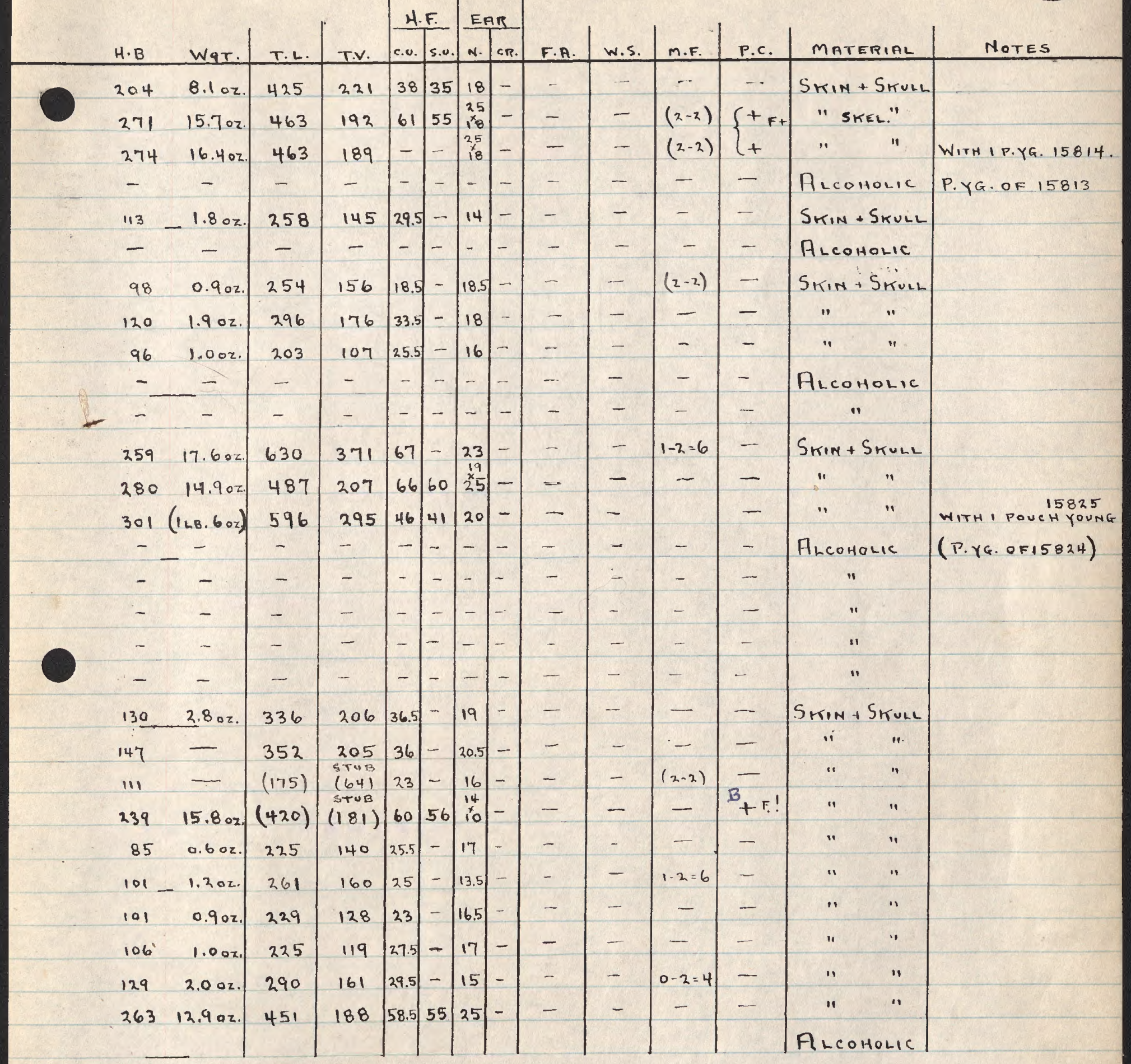




\begin{tabular}{|c|c|c|c|c|c|}
\hline $\begin{array}{l}\text { FIELD } \\
\text { No. }\end{array}$ & Name & SEX & $\begin{array}{l}1959 \\
\text { mo. }{ }_{\text {Dar }}\end{array}$ & $121 T Y$ & $E_{L E V}$ \\
\hline 15841 & MELOMYS ${ }^{192348}$ (G.8.) & & Fuey 19 & T.N.G. MT. WILHELM; PENGAGL CR. & \\
\hline
\end{tabular}

\begin{tabular}{|c|c|}
\hline 44 & "1 \\
\hline 15845 & " \\
\hline 46 & " \\
\hline 47 & " \\
\hline 48 & " \\
\hline 49 & " \\
\hline
\end{tabular}

15850 Rattus 19244.

". $192350^{\circ}$ "

42

(

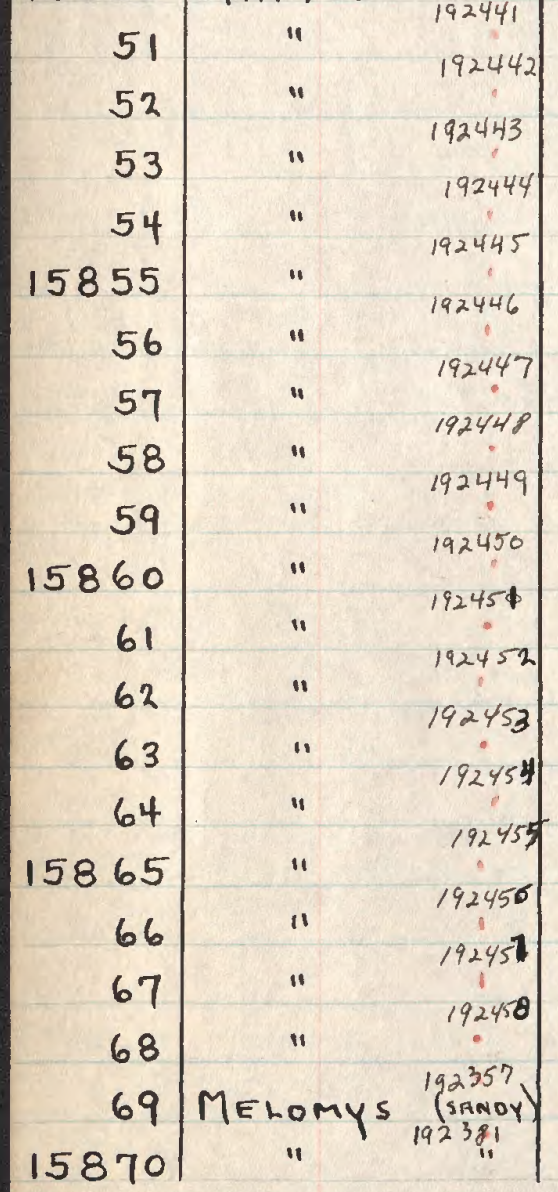

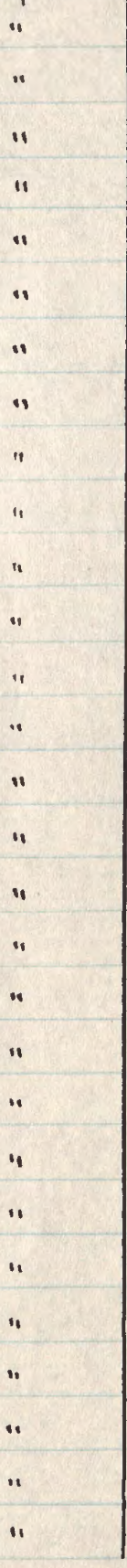

\begin{tabular}{|c|c|c|}
\hline " & " & " \\
\hline$"$ & " & " \\
\hline " & ". & " \\
\hline ". & ". & ". \\
\hline ". & “" & $"$ \\
\hline " & $"$ & ". \\
\hline ". & ". & $"$ \\
\hline . & ". & $"$ \\
\hline$"$ & $"$ & $"$ \\
\hline$"$ & $"$ & $"$ \\
\hline " & $"$ & $"$ \\
\hline$"$ & $"$ & $n$ \\
\hline$"$ & " & " \\
\hline$"$ & " & $"$ \\
\hline$"$ & $"$ & " \\
\hline ". & $"$ & " \\
\hline$"$ &.. & $"$ \\
\hline$"$ & " & ". \\
\hline$"$ & ." & " \\
\hline •. & $"$ & $"$ \\
\hline " & " & " \\
\hline . & $"$ & " \\
\hline " & $\because$ & $"$ \\
\hline .. & $"$ & $"$ \\
\hline ". & " & " \\
\hline " & $"$ & ". \\
\hline 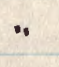 & . & " \\
\hline$"$ & $"$ & ERST SLOPES \\
\hline$"$ & $"$ & $"$ \\
\hline
\end{tabular}




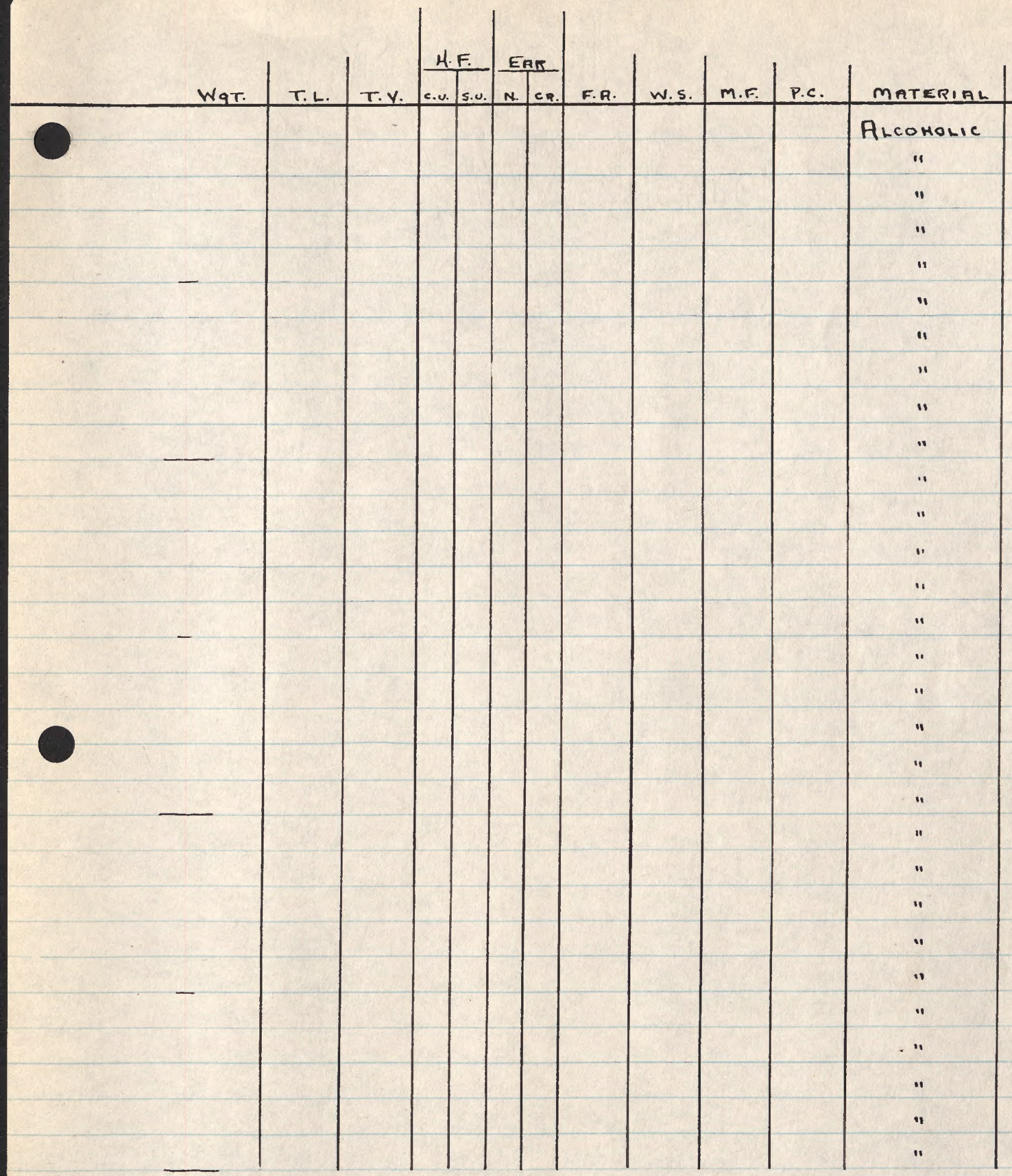






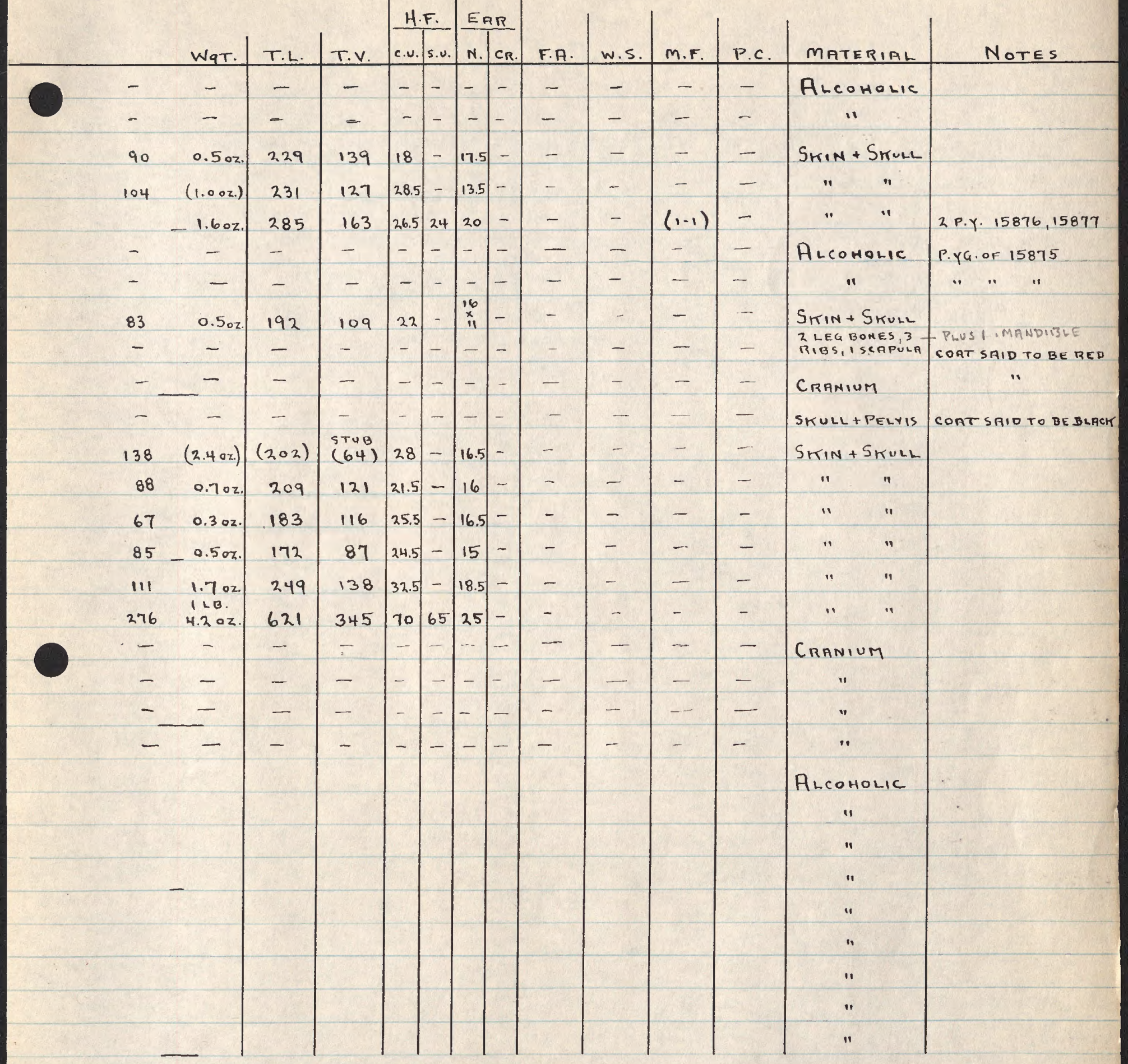






\begin{tabular}{|c|c|c|c|c|c|c|c|}
\hline $\begin{array}{c}\text { FIELD } \\
\text { No. }\end{array}$ & NAME & \multirow[t]{2}{*}{ SEX } & $\begin{array}{l}1959 \\
\text { Mo. Dar }\end{array}$ & \multicolumn{3}{|c|}{ LOCALITY } & ELEV. \\
\hline 15901 & Tattus 192460 & & Juky 20 & T.N.G & & ELm; Pengagl lr. & \\
\hline 2 & "11 192461 & & " & $"$ & " & 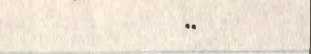 & \\
\hline 3 & 1192462 & & $"$ & $"$ & $"$ & * & \\
\hline 4 & "1 192463 & & $"$ & $"$ & " & $"$ & \\
\hline 15905 & "192464. & & $"$ & $"$ & " & “ & \\
\hline 6 & "192465. & & $"$ & $"$ & $"$ & * & \\
\hline 7 & 192466 & & $"$ & $"$ & $"$ & * & \\
\hline 8 & 192467 & & $"$ & $"$ & $"$ & 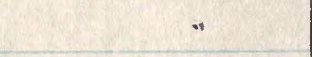 & \\
\hline 9 & " $\quad 192468$ & & $"$ & $"$ & " & " & \\
\hline 15910 & " $\quad 192469$ & & $"$ & $"$ & $"$ & $"$ & \\
\hline 11 & 1192470 & & $"$ & " & $"$ & $"$ & \\
\hline 12 & 192471 & & $"$ & $"$ & $"$ & $"$ & \\
\hline 13 & " $\quad 192472$ & & $"$ & $"$ & " & $"$ & \\
\hline 14 & $\begin{array}{l}191245 \\
\text { SYCONYCTERIS }\end{array}$ & $q$ & $"$ & " & " & EAST SLOPES & \\
\hline 15915 & Rattus RUBER & $\hat{\sigma}$ & " & $"$ & $\because$ & $"$ & \\
\hline 16 & $\begin{array}{l}191631 \\
192473\end{array}$ & q & $"$ & $"$ & $"$ & $"$ & \\
\hline 17 & $"$ & & $"$ & $"$ & $"$ & $"$ & \\
\hline 18 & " & & $"$ & $"$ & $"$ & $"$ & \\
\hline 19 & $19+476$ & & $"$ & $"$ & $"$ & " & \\
\hline 15920 & $\begin{array}{l}192476 \\
\quad 192477\end{array}$ & & $"$ & $"$ & $"$ & $"$ & \\
\hline 21 & Ratrus EXULANS & & $"$ & $"$ & $"$ & " & \\
\hline 22 & 192478 & & $"$ & $"$ & $"$ & $"$ & \\
\hline 23 & "11 & ㅇ & " & $"$ & " & $"$ & \\
\hline 24 & $\begin{array}{l}\text { MYOONYCTERIS } \\
\text { MINIOPTERUS }\end{array}$ & EMB. & JuLY 21 & $"$ & $"$ & NEM NEMB̈̈YA SAVE & \\
\hline 15925 & $7=\frac{1}{191381}$ & $\delta$ & 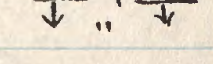 & $"$ & $"$ & $7 " 7$ & \\
\hline 26 & "191382 & 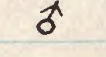 & $"$ & $"$ & $"$ & $"$ & \\
\hline 27 & "1 192986 & & $"$ & $"$ & " & $"$ & \\
\hline 28 & " 192987. & & $"$ & “ & $"$ & $"$ & \\
\hline 29 & " 192985. & & $"$ & $"$ & $"$ & $"$ & \\
\hline 15930 & "1 192988 。 & & $"$ & $"$ & $"$ & $n$ & \\
\hline
\end{tabular}




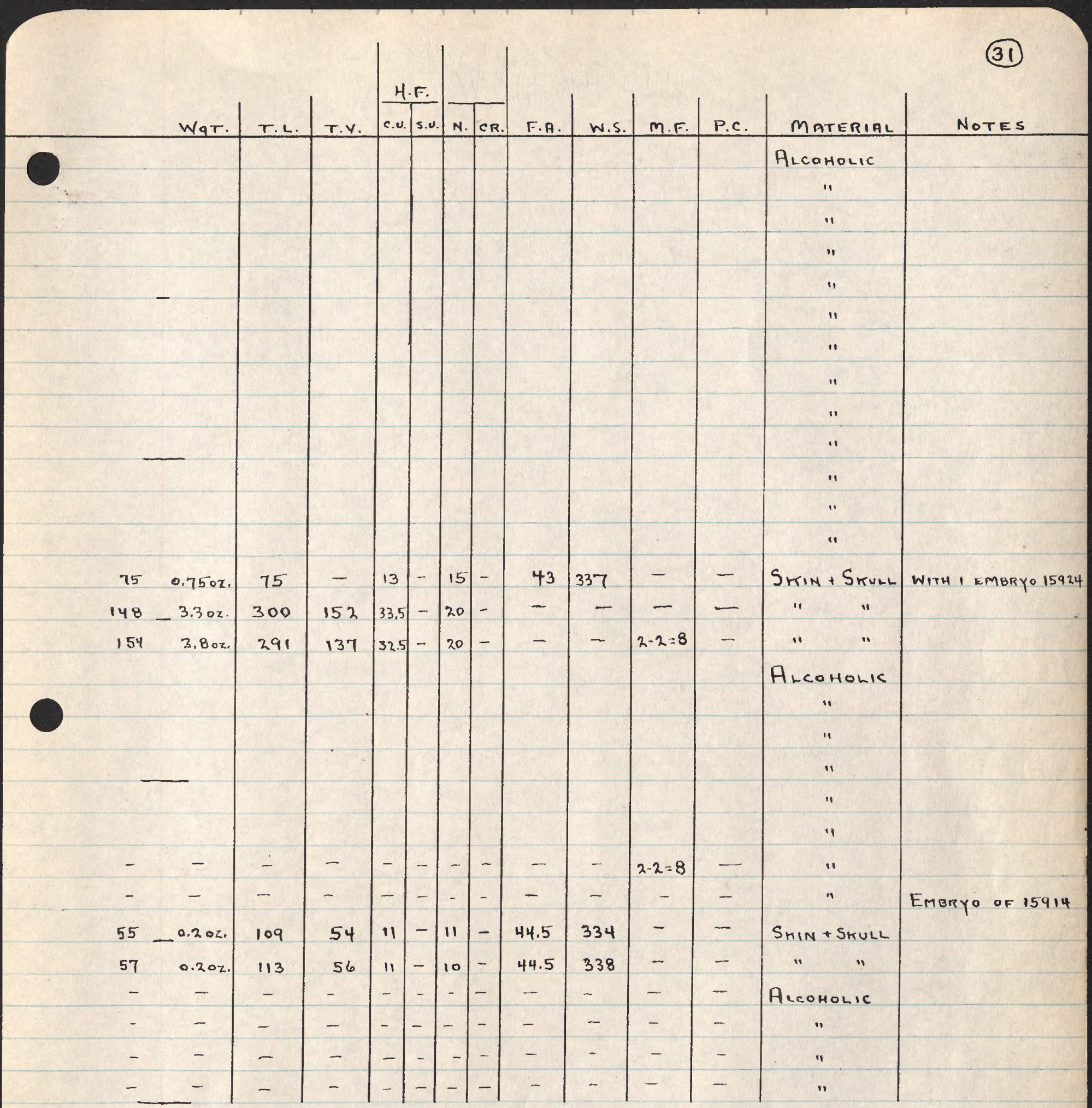


-

- 



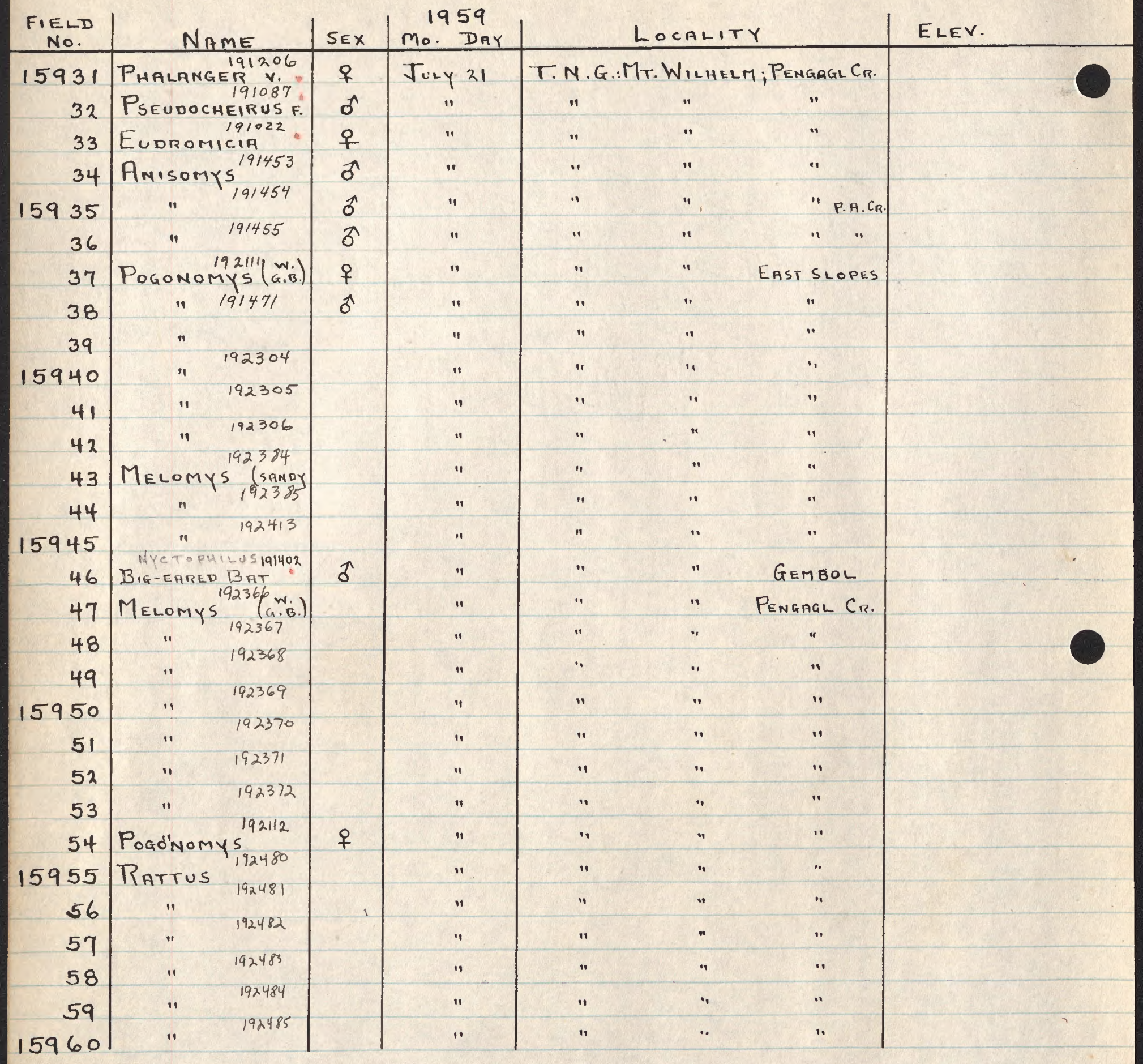




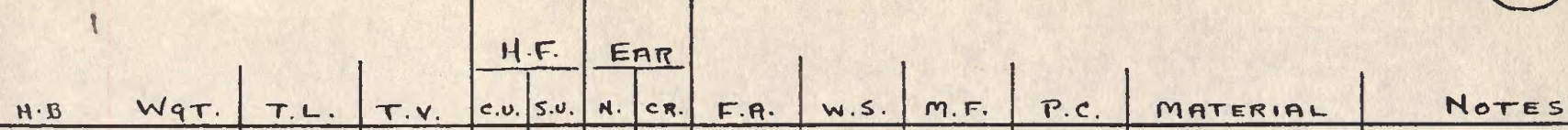

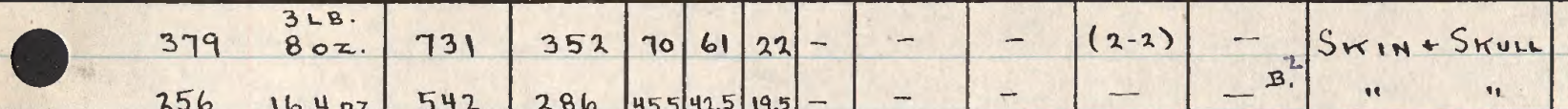

$\begin{array}{llllllll}256 & 16.402 & 542 & 286 & 45.5 & 42.5 & 19.5 & -\end{array}$

\begin{tabular}{lll|l|l|l|l|l|l}
93 & $0.750 z$ & 243 & 150 & 19 & -16.5 & -
\end{tabular}

\begin{tabular}{ll|l|l|l|l|l|l|l}
255 & 17.802 & 578 & 323 & 65 & 60 & 23 & -
\end{tabular}

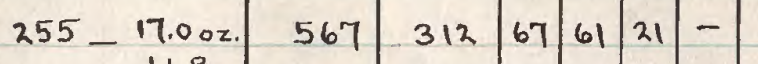

261 5.10Z. $611 \quad 350 \quad 70.564 .523$ -

$110 \quad 1.20 z .286$

$17626.5-15-$

$110 \quad 1.20 z$

$287 \quad 17726.5-15$

47

0.207

96

$498-\begin{aligned} & 26 \\ & 14 \\ & 14\end{aligned}-$

39.5

296

$-$

$109 \quad 1607$

277

168

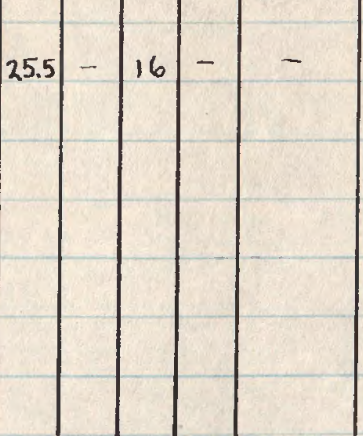

$-$

$1-2=6$

SkIN + SKULL

Ancollolic

i"

"

,

"

"1

"1

"1

SkIN + SHULL

Alcoholic

1

is

"

"

".

109.1 .607 
-

f)

,

-

- 


\begin{tabular}{|c|c|c|c|c|c|c|c|}
\hline $\begin{array}{c}\text { FIELD } \\
\text { NO. }\end{array}$ & NAME & SEX & $\begin{array}{c}1959 \\
\text { M.. Dar }\end{array}$ & \multicolumn{3}{|c|}{ LOCALITY } & ELEV. \\
\hline 15961 & RATTUS 192486 & & Juky 21 & T.N.G & & ELM; Pengagl $C_{R}$. & \\
\hline 62 & $\begin{array}{l}192481 \\
=\quad 192488\end{array}$ & & $"$ & $"$ & $"$ & " & \\
\hline 63 & " 192488 & & $"$ & $"$ & $"$ & " & \\
\hline 64 & "192484 & & " & $"$ & $"$ & $"$ & \\
\hline 15965 & 11 192490 & & $"$ & $"$ & $"$ & $"$ & \\
\hline 66 & "1 $19 \times 491$ & & $"$ & $"$ & $"$ & $"$ & \\
\hline $15970 \square$ & Phalanger 191204 & 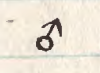 & Jumy 22 & $"$ & $"$ & $"$ & \\
\hline 1597168 & AntechinUS 190913 & $q$ & $"$ & $"$ & $"$ & $"$ & \\
\hline 1597267 & "190914 .. & 오 & $"$ & $"$ & $"$ & EAST SLOPES & \\
\hline 15970 & $\begin{array}{c}192990 \\
\text { MinIOPTERUS (57) }\end{array}$ & & Juey 21 & $"$ & $"$ & BÁYA CAVE" & \\
\hline 年8 & " $192991^{\circ}=$ & & $"$ & $"$ & $"$ & $"$ & \\
\hline$\frac{69}{72}$ & 1192989 " & & $"$ & $"$ & 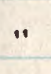 & $"$ & \\
\hline 73 & $\begin{array}{c}191149 \\
\text { PSEUDOCHEIRUS CUP. }\end{array}$ & $\hat{\sigma}$ & JuLy 22 & $"$ & $"$ & PengRGL CREEK & \\
\hline 74 & Melomys 192023 (G.) & $\hat{\sigma}$ & $"$ & $"$ & " & $"$ & \\
\hline 15975 & PogOnOMYS 192307 & & $"$ & $"$ & " & $"$ & \\
\hline 76 & Ratius 191781 & $q$ & 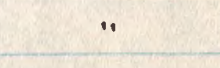 & $"$ & " & $"$ & \\
\hline 77 & $\begin{array}{r}192492 \\
\because \quad 192493\end{array}$ & & $"$ & $"$ & $"$ & $"$ & ) \\
\hline 78 & " 192445 & & $"$ & $"$ & " & " & \\
\hline 79 & " & & " & $"$ & $"$ & $"$ & \\
\hline 15980 & $"$ & & $"$ & 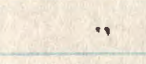 & $"$ & " & \\
\hline 81 & $\because \quad 191244$ & & $"$ & " & $"$ & " & $p$ \\
\hline 82 & SYCONYCTERIS & $\hat{\sigma}$ & JuLy 23 & $"$ & $"$ & EAST SLOPES & \\
\hline 83 & 1191246 & 우 & $"$ & " & $"$ & $"$ & \\
\hline 84 & Petaurus 191060 & 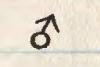 & $"$ & $"$ & " & " KENKV & \\
\hline 15985 & PSEUDOHYDROMYS & $\hat{\sigma}$ & $"$ & $"$ & " & iPengagh $C_{R}$. & \\
\hline 86 & MeLOMYS ${ }^{192024}\left(\begin{array}{l}\text { W. } \\
\text { G.B. }\end{array}\right)$ & $\hat{s}$ & $"$ & $"$ & $"$ & 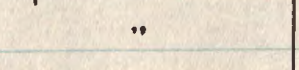 & \\
\hline 87 & MELOMYS (SANDY & $\delta$ & $"$ & " & $"$ & EAST SLGPES & \\
\hline 88 & RAtrus RUBER & $\hat{\sigma}$ & $"$ & 4 & $"$ & $"$ & \\
\hline 89 & Melomys (FAWM) & $q$ & " & $"$ & $"$ & $n$ & \\
\hline 159901 & Pogonomys (WH.B.) & $\delta$ & " & " & " & $"$ & \\
\hline
\end{tabular}


H.F. EAR \begin{tabular}{l|l|l} 
WaT. & T.L. & T.V. \\
\hline
\end{tabular}

c.u. S.U. N. r.

w.s.
\begin{tabular}{l|l|l|l|} 
M.F. & P.C. & MATERIAL & NOTES
\end{tabular}

$\begin{array}{cc}- & - \\ - & - \\ - & - \\ - & - \\ - & - \\ 380 & 420 \\ 302\end{array}$

$1010.80 z$.

$108 \quad 0.80 z$.

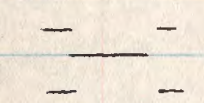

$362 \quad 34 B$.

$116 \quad 1.7 \mathrm{oz}$.

680 261

${ }_{100}^{-}-\frac{1.00 z}{}$

213

$$
-
$$

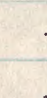

$73 \quad 0.602$.

$73 \quad 0.40 x$

$124 \quad 1.802$.

$95-0.60 z .180$

$120 \quad 2.10 z .265$

127 2.10z. 284

$\begin{array}{ll}150 & 3.302 \quad 300\end{array}$

137 3.4.2. 327

$135 \quad 3.20 z .1350$

43

\begin{tabular}{c|c}
- & - \\
- & - \\
- & -
\end{tabular}

$-D_{-}-{ }_{-}-$

$-{ }_{-}^{-}$

$-{ }_{-}^{-}--$

$-$

36

69

139

135

$-\quad-$

$-$

318

$14532.5-17.5-$

\begin{tabular}{l|l|l}
- & - & - \\
113 & 26.5 & - \\
- & 17 & -
\end{tabular}

$-{ }_{-}^{-}$

$-$

$-$

$-$

-

-

$152 \quad 26 \quad 2321$ -

8524 -

145

$15731.5-15.5-$

$150 \quad 33-19-$

190

$215|29|-|15|-1$

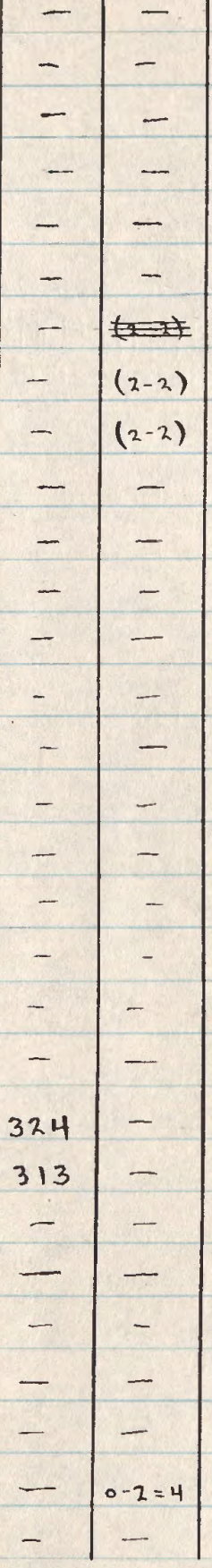

- $\begin{gathered}\text { AlCOHOLIC } \\ \text { - } \\ \text { " }\end{gathered}$

-

-

- Skin+ SkULL

"

"

AlCOHOLIC

- "

- $\mid \begin{gathered}\text { - } \\ \text { Krin }+S_{\text {KuLl }}\end{gathered}$

- $"$ "

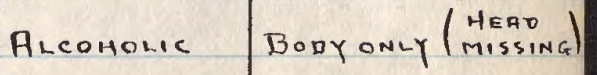
STIAT SKULL (WHITISH BELLY)

AlCOHOLIC

"I

"

“

"

SKIN + SKULL

"

"

"Bopy

$\begin{array}{ll}1 & \text {. } \\ 1 & \text {. }\end{array}$

$\begin{array}{cc}11 & 1 \\ 11 & \ldots \\ \text { " } & \text {. }\end{array}$

15982,3: FUR ON WIMG MEMBRAHE LIGHTER (CONTRASTS) BROMN THAN BACK PELAGE MEMBRANE IMSERTION ON H.F. ALONG INSIDE EDGE OVER I ST TOE 



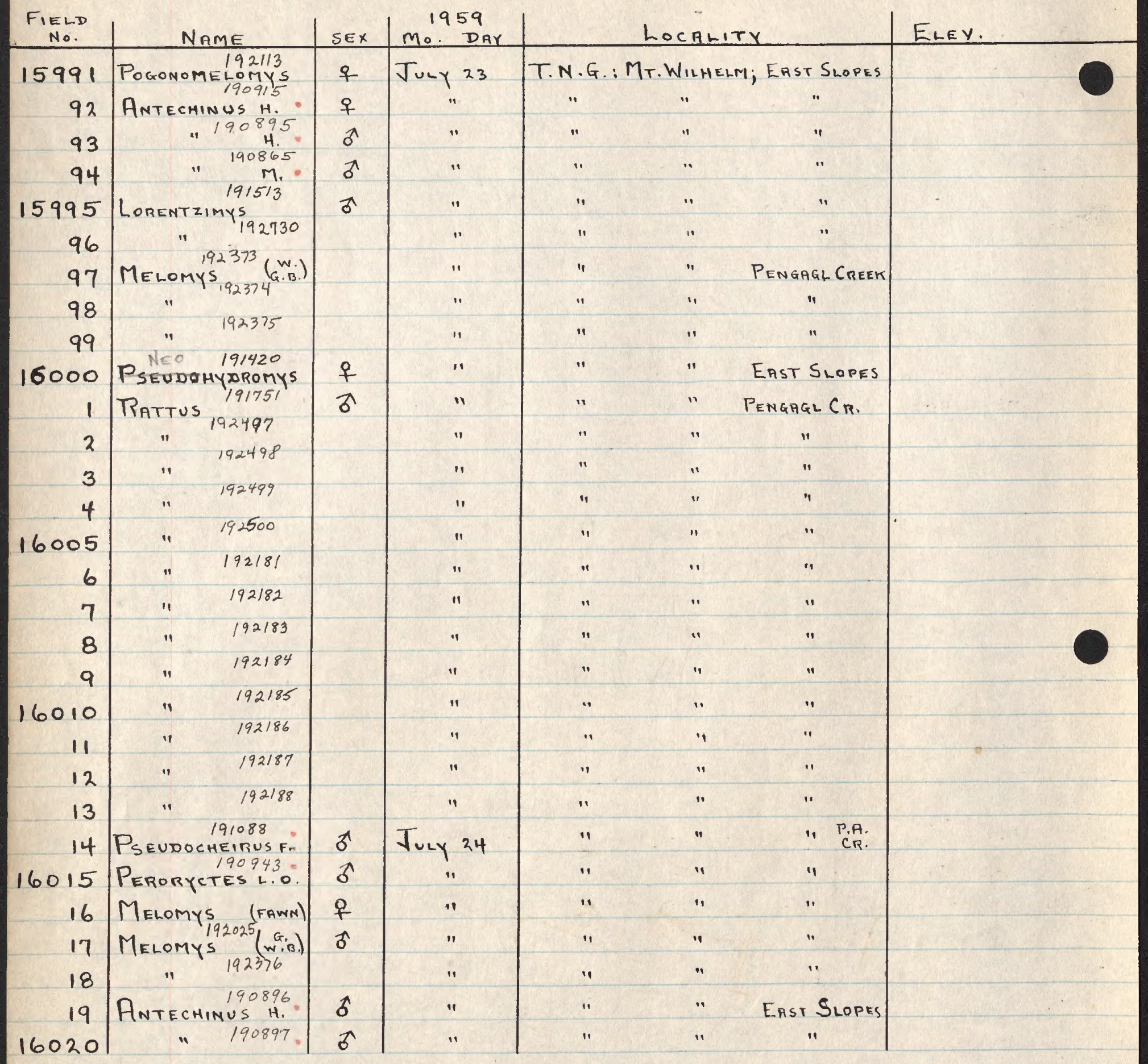


H.B. WGT. T.L. T.V. C.U. T.F.

\begin{tabular}{l|l|l|l} 
F.A. & W.S. & M.F. & P.C
\end{tabular}

.

MATERIAL Notes

$(90)$ $104 \quad 0.90 z$. 9 $99 \quad 0.902$. 73

- 0.30

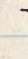

9

$95 \quad 0.7502 .179$

$115 \quad 1.402 .242$

244

140

221

125

235

136

$25.5-17$

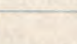

-

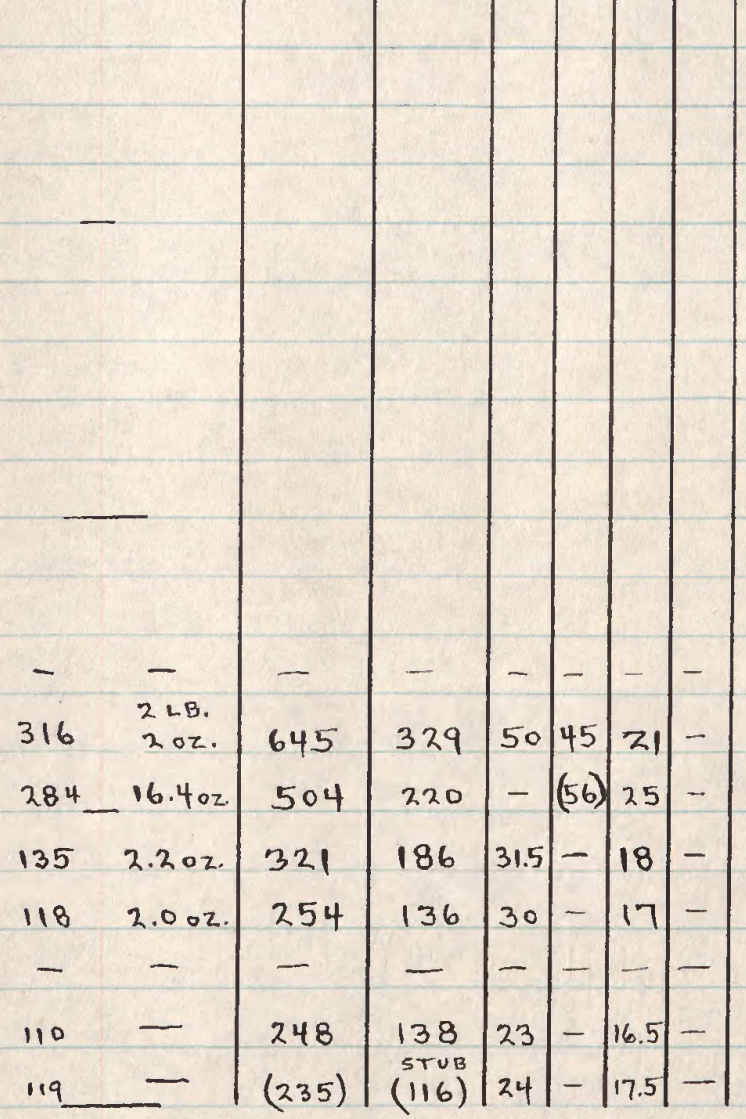





\begin{tabular}{|c|c|c|c|c|c|c|c|}
\hline $\begin{array}{c}\text { FIELD } \\
\text { NO. }\end{array}$ & NAmE & SEX & $\begin{array}{c}1959 \\
\text { Mo. Dar }\end{array}$ & \multicolumn{3}{|c|}{ LOCALITY } & ELEVATION \\
\hline 16021 & Pipistrellatis & $\vec{\sigma}$ & JuLy 24 & $T \cdot N \cdot G$ & $W_{1}$ & EM; EAST SLOPES & \\
\hline 22 & AnIsomys $^{192732}$ & q & $"$ & $" 1$ & $"$ & Pengagl CreEk & \\
\hline 23 & RATtus RUBER & q & $"$ & $"$ & " & East SLOPES & \\
\hline 24 & " $\quad 191782$ & q & $"$ & $"$ & $"$ & " & \\
\hline 16025 & MELOMYS "GOO" & q & $"$ & $"$ & $"$ & $"$ & \\
\hline 26 & " & 오 & $"$ & $"$ & $"$ & $"$ & \\
\hline 27 & $"$ & f $1 \mathrm{.}$ & $"$ & $"$ & " & n & \\
\hline 28 & " & arm. & $"$ & " & $"$ & $"$ & \\
\hline 29 & $\begin{array}{l}191011 \\
\text { EUDROMICIA }\end{array}$ & $\delta$ & $"$ & $"$ & " & $"$ & \\
\hline 16030 & SYCONYCTERIS & $\hat{\sigma}$ & $"$ & $"$ & $"$ & " & \\
\hline 31 & Rattus 192189 & $\hat{\sigma}$ & $"$ & " & $"$ & Pengagl Creek & \\
\hline 32 & 192190 & $\hat{d}$ & $"$ & $" 1$ & $"$ & " & \\
\hline 33 & 192191 & $\hat{\sigma}$ & $"$ & $" 1$ & $"$ & " & \\
\hline 34 & 192192 & $\hat{\jmath}$ & $"$ & $"$ & " & $"$ & • \\
\hline 16035 & 192193 & a & $"$ & $"$ & $"$ & 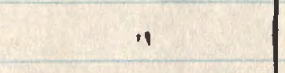 & \\
\hline 36 & 192194 & $\hat{\sigma}$ & $"$ & $" 1$ & $"$ & " & \\
\hline 37 & 192195 & $\hat{\sigma}$ & $"$ & $"$ & " & " & \\
\hline 38 & 192196 & $q$ & $"$ & $"$ & " & " & \\
\hline 39 & 192197 & q & $"$ & $"$ & $"$ & $"$ & \\
\hline 16040 & 192198 & q & $"$ & $"$ & $"$ & $"$ & \\
\hline 41 & 192199 & 우 & $"$ & $"$ & $"$ & $"$ & \\
\hline 42 & 192200 & $q$ & $"$ & $"$ & $"$ & $"$ & \\
\hline 43 & 192201 & 웅 & $"$ & $"$ & $"$ & $"$ & \\
\hline 44 & 192202 & o $\mathrm{im}$. & $"$ & $"$ & " & $"$ & \\
\hline 16045 & 192203 & \& $\quad$ im. & $"$ & $"$ & $"$ & $"$ & \\
\hline 46 & $" 192204$ & $f \quad \mathrm{~m}$ & $"$ & $"$ & $"$ & $"$ & \\
\hline 47 & $\begin{array}{l}192205 \\
193206\end{array}$ & \& $\mathrm{im}$ & $"$ & $"$ & " & $"$ & $<$ \\
\hline 48 & 192206 & के & $"$ & $"$ & $" 1$ & " & \\
\hline 49 & 192207 & 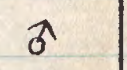 & $"$ & $"$ & 11 & $"$ & \\
\hline 16050 & 192208 & $\delta$ & $"$ & $"$ & " & 11 & 8 \\
\hline
\end{tabular}




-

-

- 
(36)

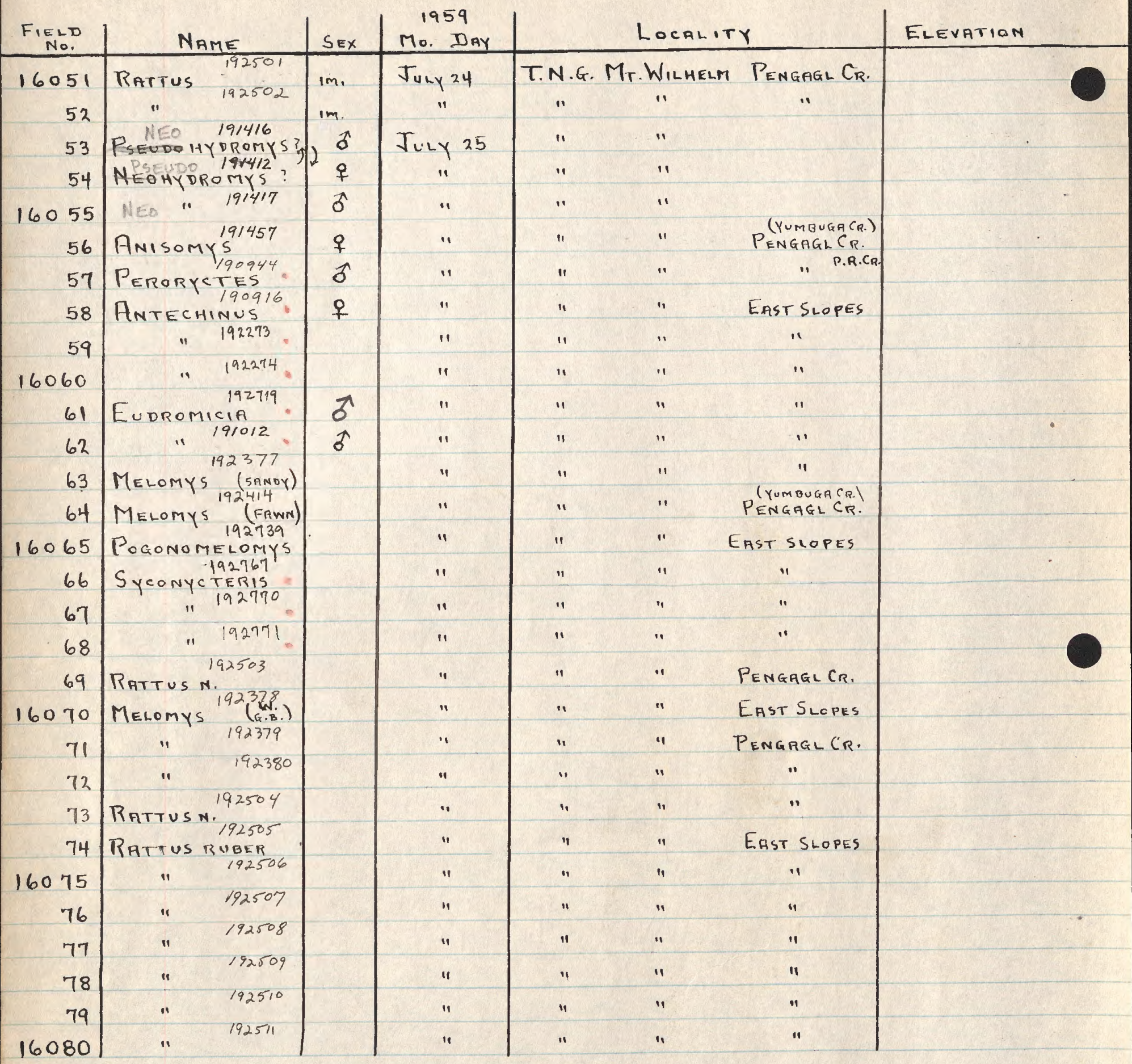

1080 


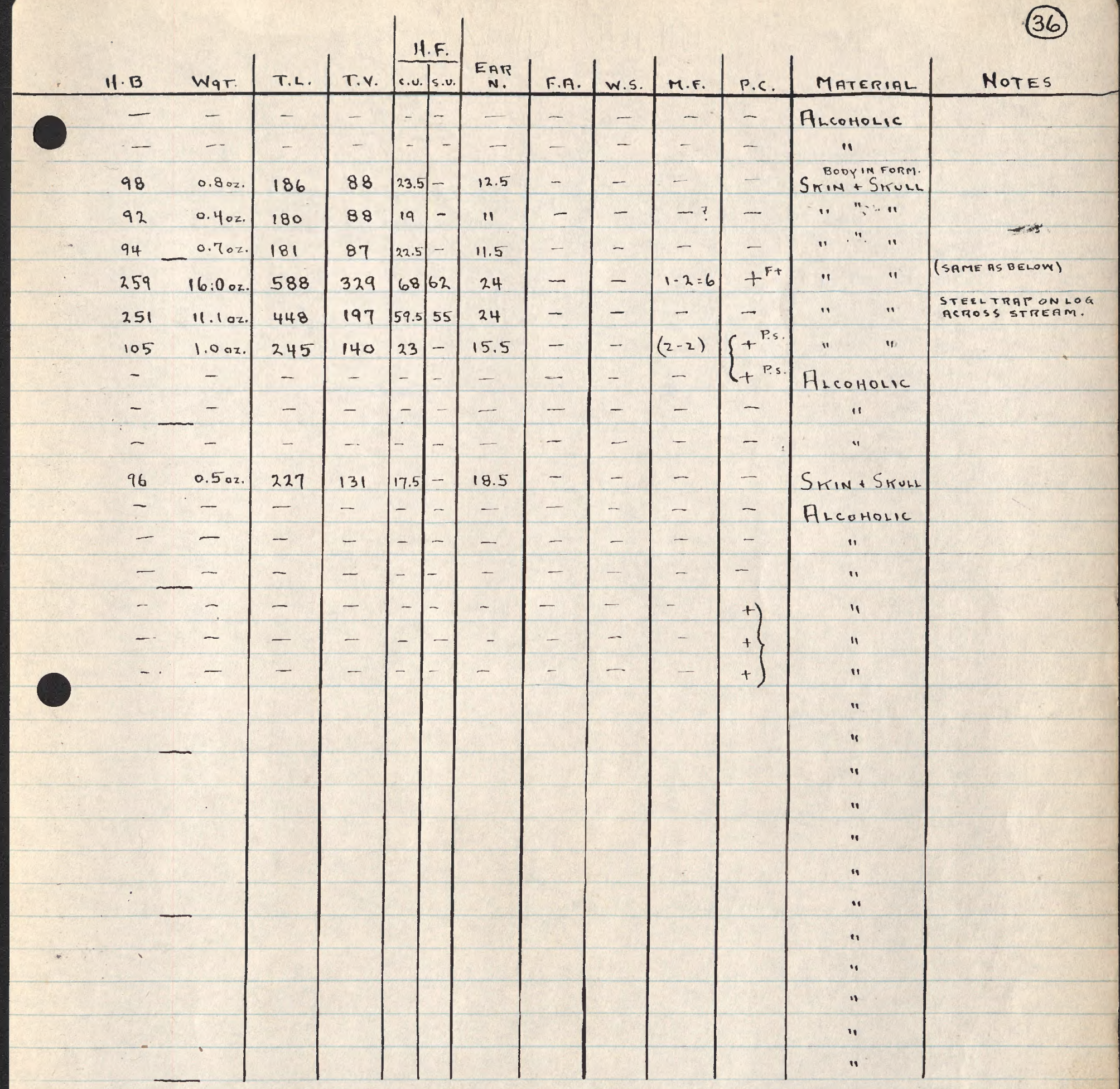



-

○

○ 
(37)

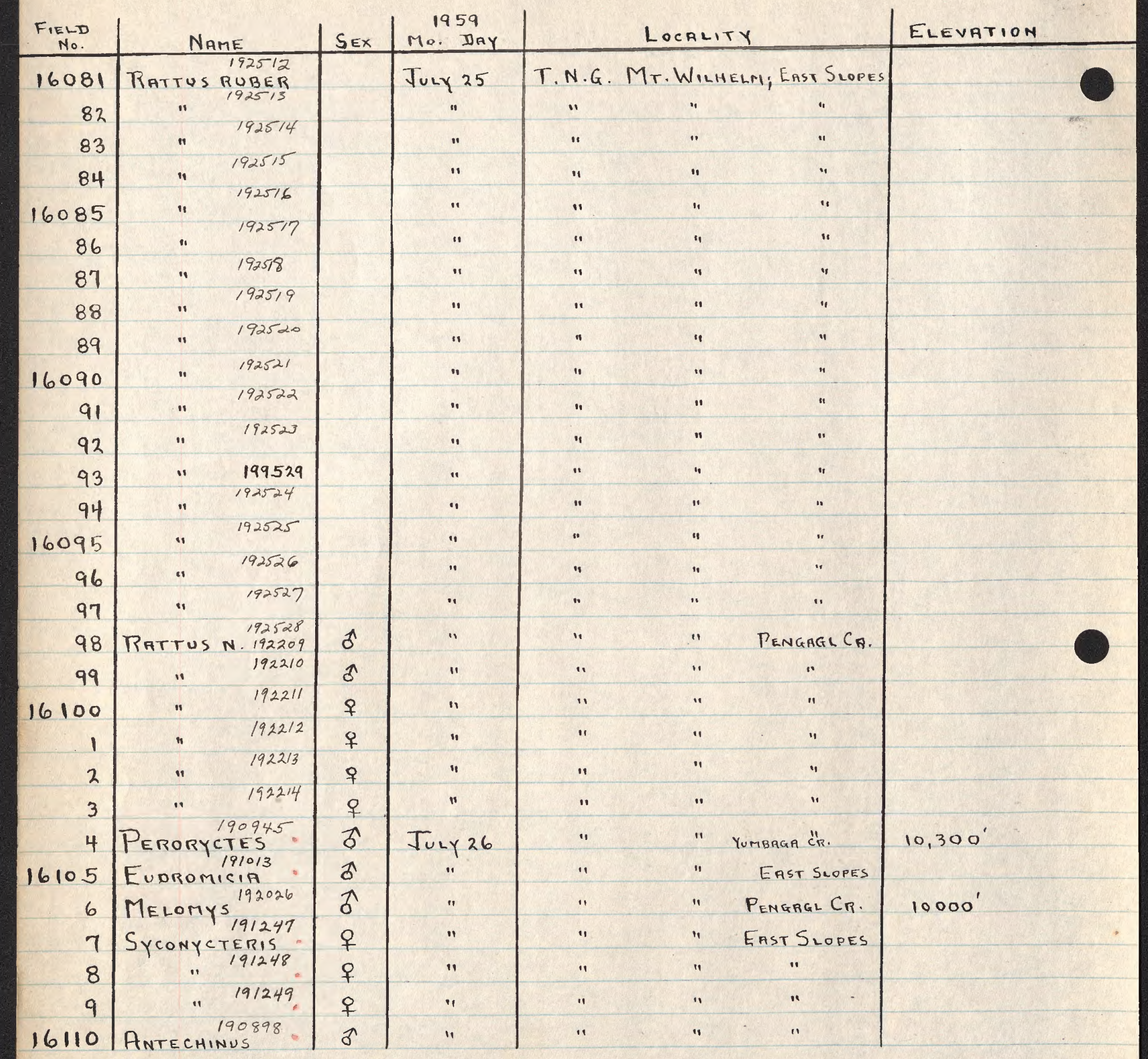


(37)

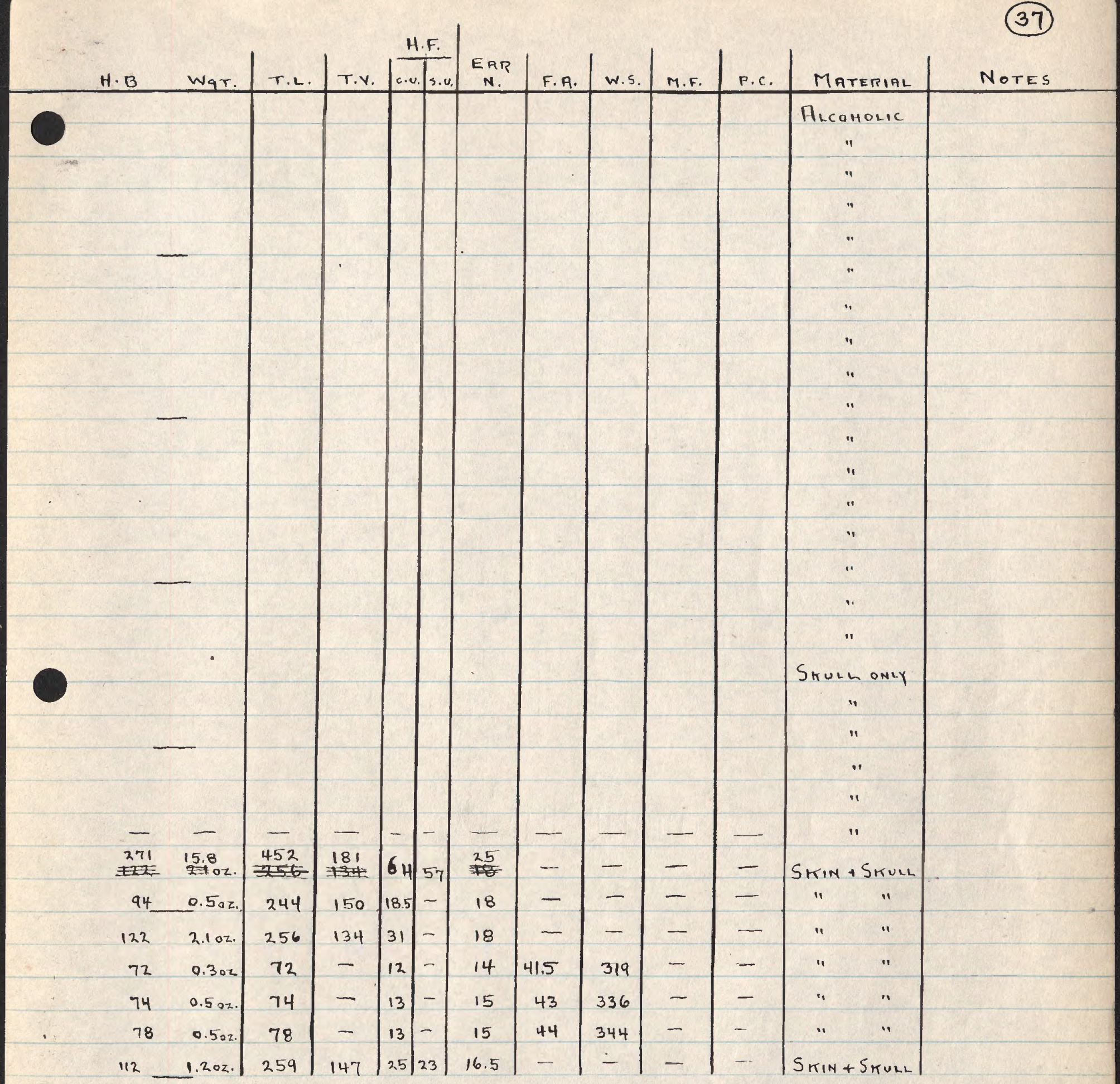


-

-

- 
(38)

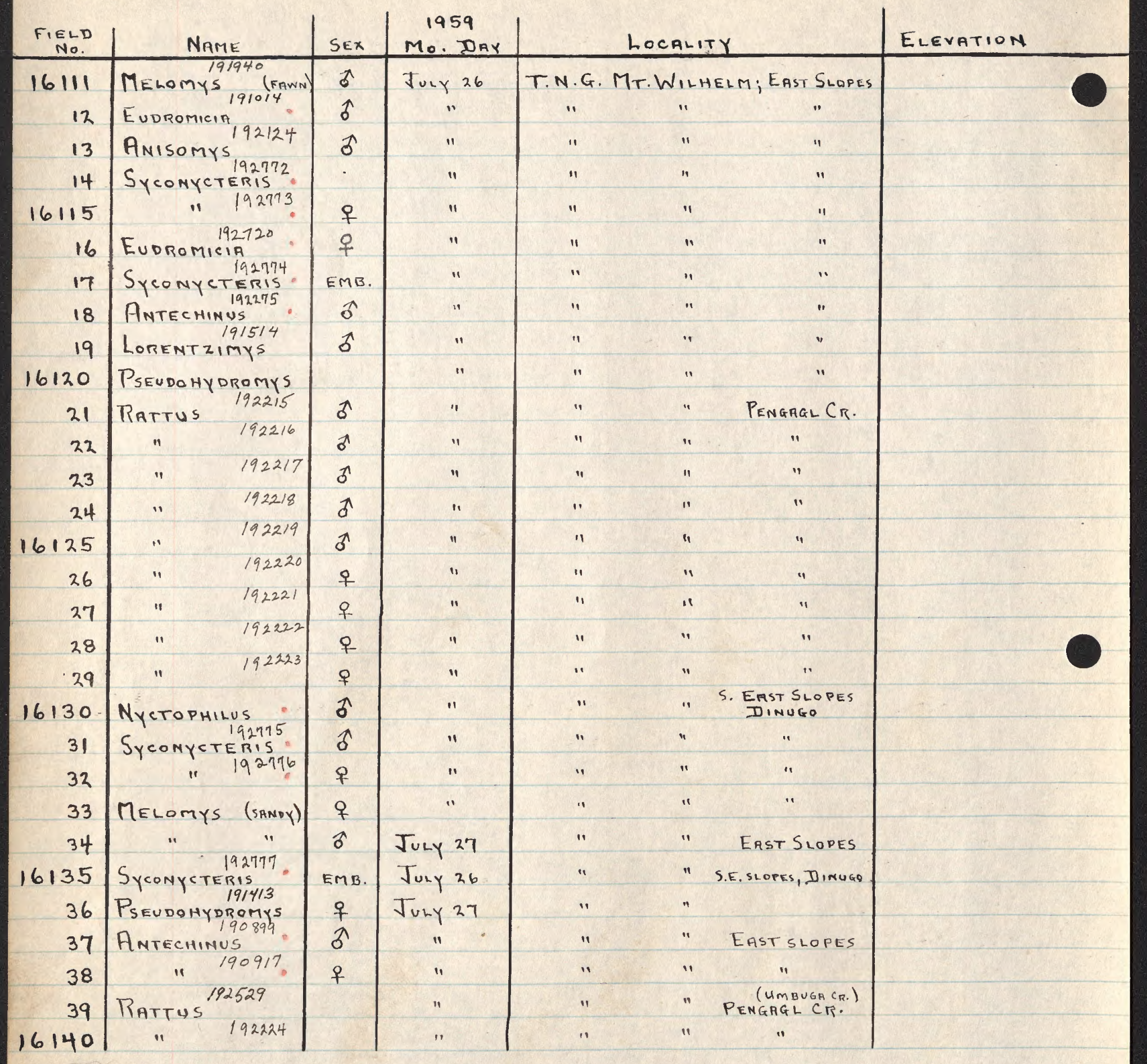




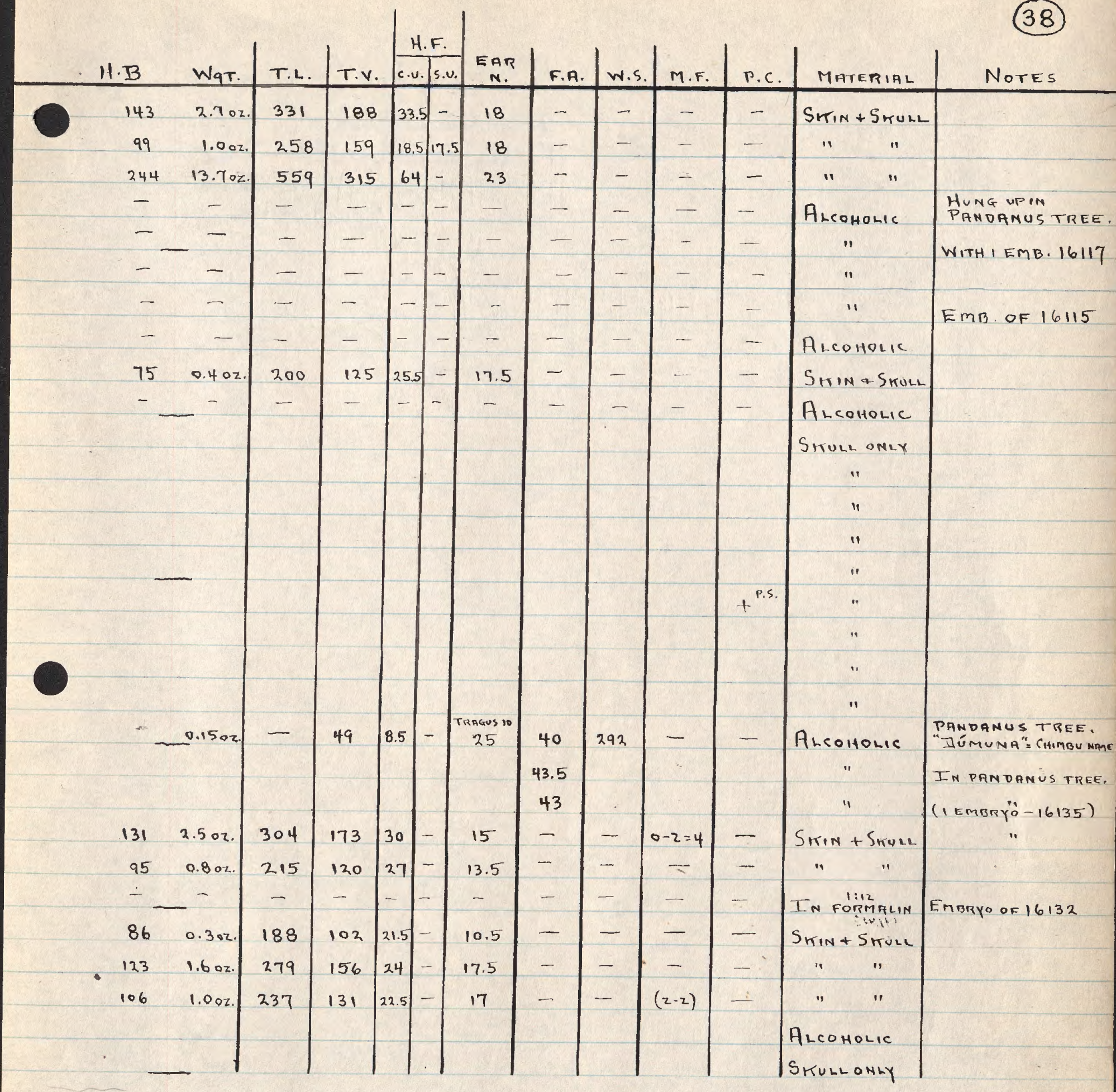




\section{.}





\begin{tabular}{|c|c|c|c|c|c|c|c|}
\hline $\begin{array}{l}\text { FIELD } \\
\text { No. }\end{array}$ & NAmE & SEX & $\begin{array}{c}1959 \\
\text { Mo. DAY }\end{array}$ & \multicolumn{3}{|c|}{ LOCALITY } & Elevation \\
\hline 16141 & Ratrus 191783 & 우 & JuLy 27 & T.N.G & & 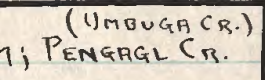 & \\
\hline 42 & ANISOMYS? & $q$ & $"$ & $"$ & " & $"$ & \\
\hline 43 & RATTUS 192225 & s & $"$ & $"$ & " & " & \\
\hline 44 & " 192226 & $\hat{\sigma}$ & $"$ & $"$ & " & $"$ & \\
\hline 16145 & 192227 & $\delta$ & $"$ & $"$ & " & " & \\
\hline 46 & $" 192228$ & q & $"$ & " & " & $*$ & \\
\hline 47 & SYCONYCTERIS & 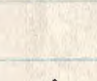 & $"$ & $"$ & " & EAst SLOPES & \\
\hline 48 & NyctopHILUS = & $\hat{\sigma}$ & " & $"$ & $"$ & Waimambuno & \\
\hline 49 & 11 191403. & $\widehat{\sigma}$ & $"$ & $"$ & $"$ & $"$ & \\
\hline 16150 & MELOMYS (sangY) & 6 & " & $"$ & $"$ & EAst SLutes & \\
\hline 51 & PSEUDOCHEIRUS F: & $\hat{\sigma}$ & Juky 28 & $"$ & " & Pengagl $\mathrm{Cr}$. & \\
\hline 52 & MELOMYS (SANOY) & $\hat{\sigma}$ & $"$ & " & " & EAST SLOPES & \\
\hline 53 & Miniopterus (s) & & $"$ & $"$ & " & " & \\
\hline 54 & " 192994. & & " & $"$ & $"$ & " & \\
\hline 16155 & $=192992$. & & $"$ & $"$ & " & $"$ & \\
\hline 56 & PerReryctes 19.0. & $q$ & $"$ & " & " & PENGAGL CR. & \\
\hline 57 & Pseudohyorom 191414 & 우 & " & $"$ & & 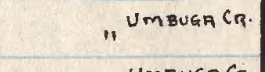 & \\
\hline 58 & Antechinus & 6 & $"$ & $"$ & $"$ & $"$ UMBUGA & \\
\hline 59 & MeLOMYS $19204 /$ (G.1.). & q & $"$ & $"$ & $"$ & $"$ & $=$ \\
\hline 16160 & NEOHYOROMYS 191423 & $\widehat{d}$ & " & $"$ & " & $"$ & $c$ \\
\hline 61 & 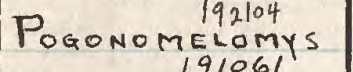 & $\hat{\sigma}$ & $"$ & " & $"$ & EAST SLOPES & \\
\hline 62 & Petaurus 191061. & $\hat{\sigma}$ & $"$ & " & " & " & \\
\hline 63 & 11.92714 & 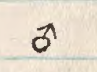 & " & $"$ & " & $"$ & \\
\hline 64 & 11 192715 . & $\hat{\sigma}$ & $"$ & $"$ & $"$ & $"$ & \\
\hline 16165 & ANTECHINUS 192276 & & $"$ & " & $"$ & $"$ & \\
\hline 66 & $\begin{array}{l}191015 . \\
\text { Eudromicin } \\
192530\end{array}$ & ఓ & $"$ & " & $"$ & $"$ & \\
\hline 67 & RATTUS RUBER & & " & " & " & " & \\
\hline 68 & Rattus 192229 & $\hat{\sigma}$ & $"$ & $"$ & $"$ & Penghat $C r$. & \\
\hline 69 & $11 \quad 192230$ & $\delta$ & $"$ & $"$ & $"$ & $"$ & \\
\hline 16170 & 192231 & $\hat{\sigma}$ & " & " & $"$ & " & \\
\hline
\end{tabular}




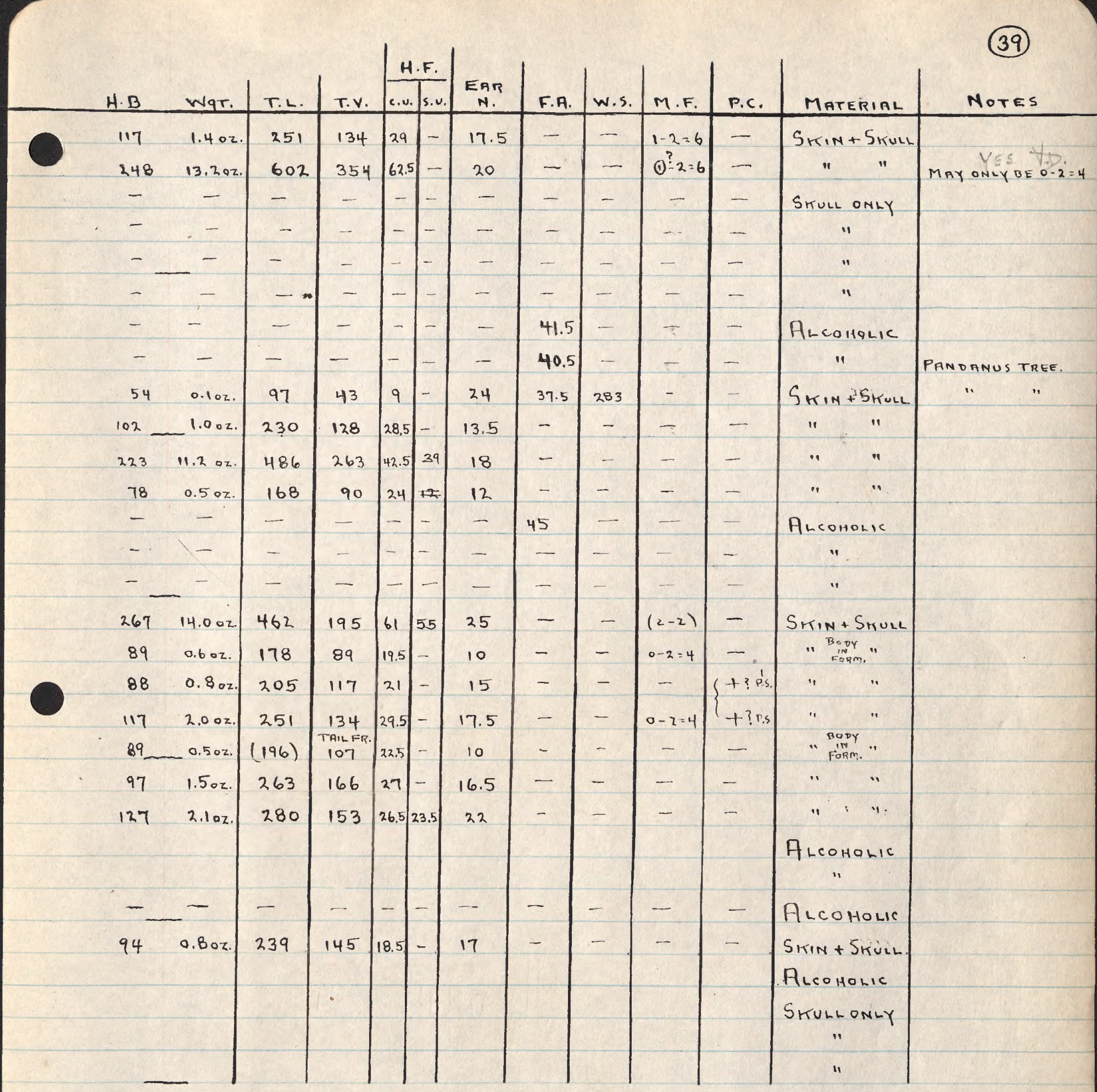

16157 

(40)

\begin{tabular}{|c|c|c|c|c|c|c|}
\hline $\begin{array}{c}\text { FieLd } \\
\text { No. }\end{array}$ & Name & SEX & $\begin{array}{l}1959 \\
\text { Mo. DAY }\end{array}$ & \multicolumn{3}{|c|}{ LecaLity } \\
\hline 16171 & Ratrus 192232 & $\hat{a}$ & JuLY 28 & T.N.G. & NILH & $M_{i} P_{\text {ENGAGL }} C_{R}$. \\
\hline 72 & $11 \quad / 92233$ & $d$ & " & " & $"$ & " \\
\hline 73 & 192234 & 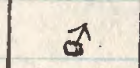 & - " ". & $"$ & $"$ & " \\
\hline 74 & 192235 & ๙ & $"$ & $"$ & $"$ & " \\
\hline 16175 & 192236 & 9 & " & " & $"$ & $"$ \\
\hline 76 & " $\quad 192237$ & 오 & $"$ & $"$ & $"$ & $"$ \\
\hline 77 & " $\quad 192238$ & ㅇ & $"$ & $"$ & " & $"$ \\
\hline 78 & " 192239 & 우 & $"$ & $"$ & $"$ & $"$ \\
\hline 79 & PeTAURUS 191062 & $\hat{\sigma}$ & TuLy29 & $"$ & " & EAST SLOPES \\
\hline 16180 & "191066 & q & 11 & $"$ & " & $"$ \\
\hline 81 & 1191067 . & 우 & $"$ & $"$ & $"$ & $"$ \\
\hline 82 & $\begin{array}{r}192769 \\
\text { Sycon'yct大is }\end{array}$ & $\frac{\frac{1}{6}}{5}$ & $" 1$ & $"$ & $"$ & " \\
\hline 83 & DENOROLAGUS 19215 & ? & (") & $"$ & $"$ & $\begin{array}{l}\text { (NR. BUNDI) } \\
\text { MONDIR }\end{array}$ \\
\hline 84 & $\begin{array}{l}192778 \\
\text { SyconYCTERIS } \\
\text { Sycis }\end{array}$ & $\sigma$ & $"$ & $"$ & " & UMBAMÁmbano \\
\hline 16185 & $\begin{array}{l}191421 \\
\text { NEOHYDROMYS }\end{array}$ & 우 & $" 1$ & " & $"$ & U'MBAGA \\
\hline 86 & $\begin{array}{l}190918 \\
\text { AntECHINUS }\end{array}$ & 우 & $"$ & $"$ & $"$ & " \\
\hline 87 & $\begin{array}{l}191422 \\
\text { NeoHyoromys }\end{array}$ & q & $"$ & " & $"$ & $"$ \\
\hline 88 & ANTECHINUIS & ठ & $"$ & $"$ & " & Pengagl $C_{R}$. \\
\hline 89 & Ratrus 192240 & $a$ & " & $"$ & " & $" 1$ \\
\hline 16190 & $\begin{array}{l}1192241 \\
\quad 192240\end{array}$ & $\delta$ & $"$ & $"$ & $"$ & $"$ \\
\hline 91 & 11 192242 & ठ & $" 1$ & $"$ & " & $"$ \\
\hline 92 & MELOMYS 192180 & $?$ & $"$ & $"$ & " & $"$ \\
\hline 93 & Rattus 192243 & q & " & $"$ & $"$ & $"$ \\
\hline 94 & 192244 & i & " & " & " & " \\
\hline 16195 & "1 $\quad 192245$ & q & $"$ & $"$ & " & $"$ \\
\hline 96 & $\begin{array}{ll}11 & 192246 \\
& 192247\end{array}$ & 우 & " & " & " & " \\
\hline 97 & 192247 & q & $"$ & 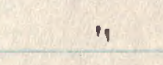 & $"$ & “ \\
\hline 98 & 192248 & q & $"$ & $"$ & $"$ & $"$ \\
\hline 99 & 192249 & im. ô? & $"$ & " & $"$ & $"$ \\
\hline 16200 & $19225^{\circ}$ & $\lim \hat{\sigma} ?$ & $"$ & " & $"$ & $"$ \\
\hline
\end{tabular}




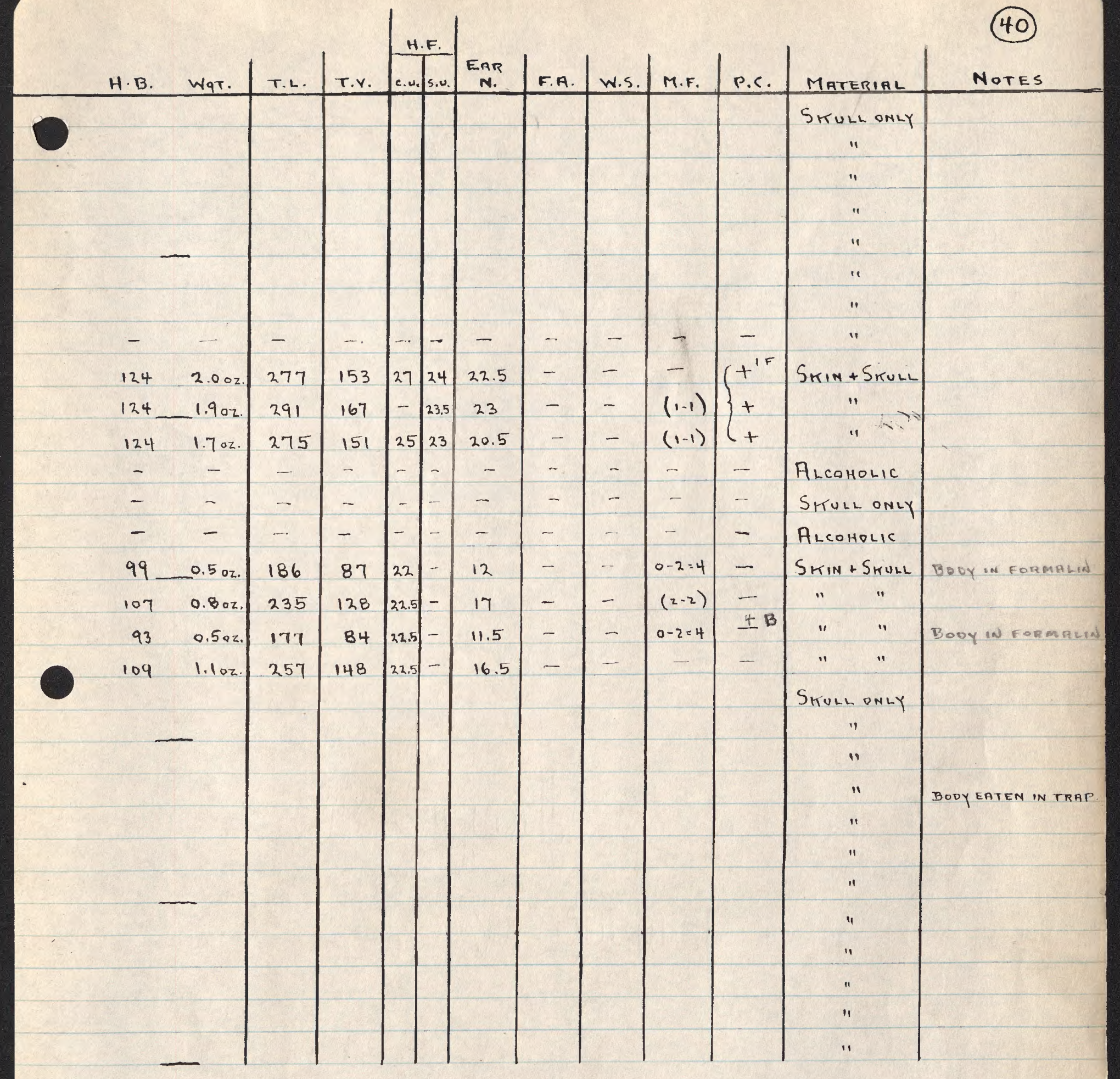



0

-

• 
(41)

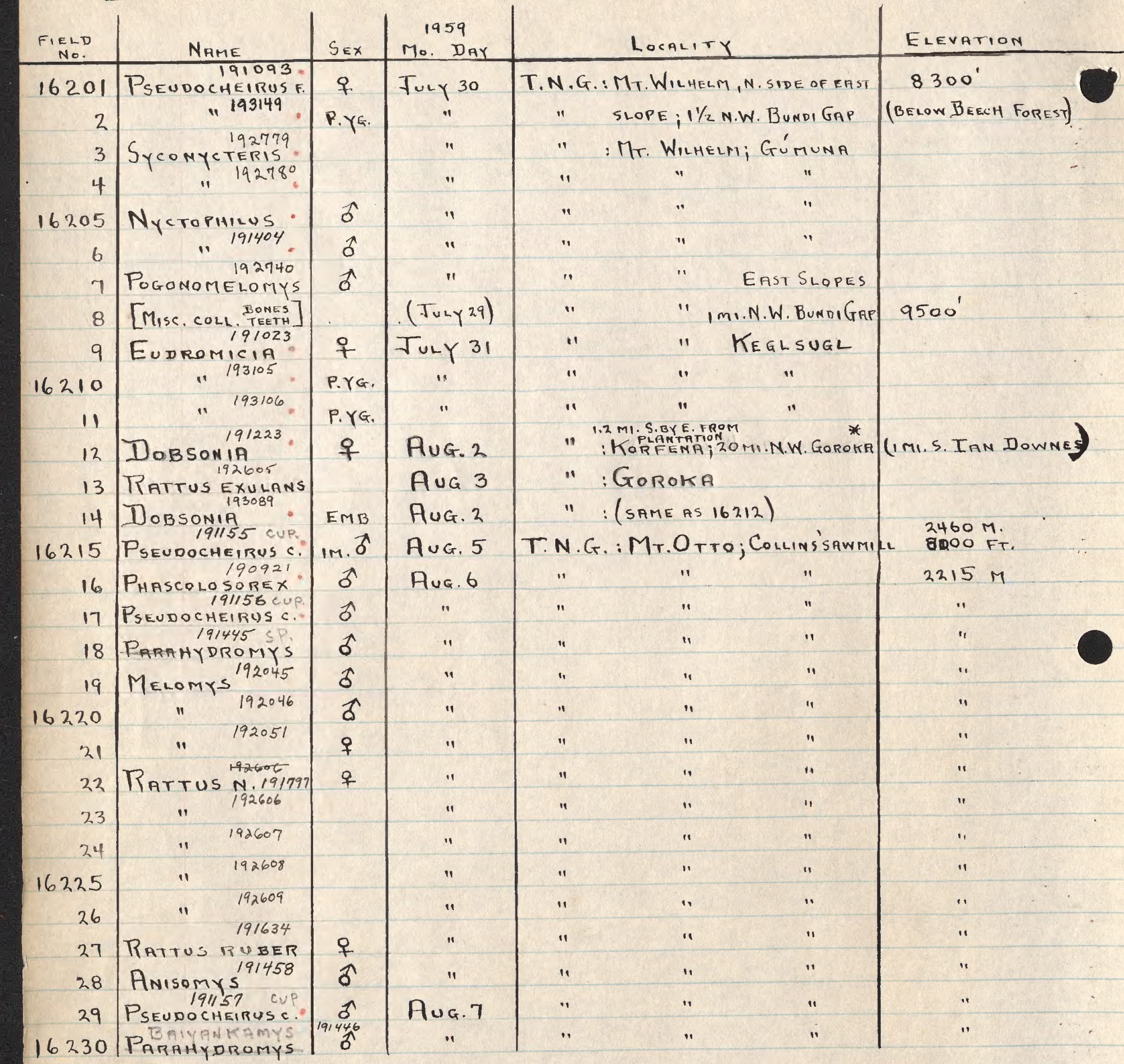

$4145 k w+5 k+L$

315 Pleollonic

MT. WILHELM - 832 MAMMALS

16212 NAME OF MOUNTAIN IN WHICH WE FOUND CAVES (NORTH FACE) - NEIMEIFA

Altitude - 5760 FEeT

* "Some 16.5 mi. W. of Goroka in the main Asaro Valley" Ian Donns 


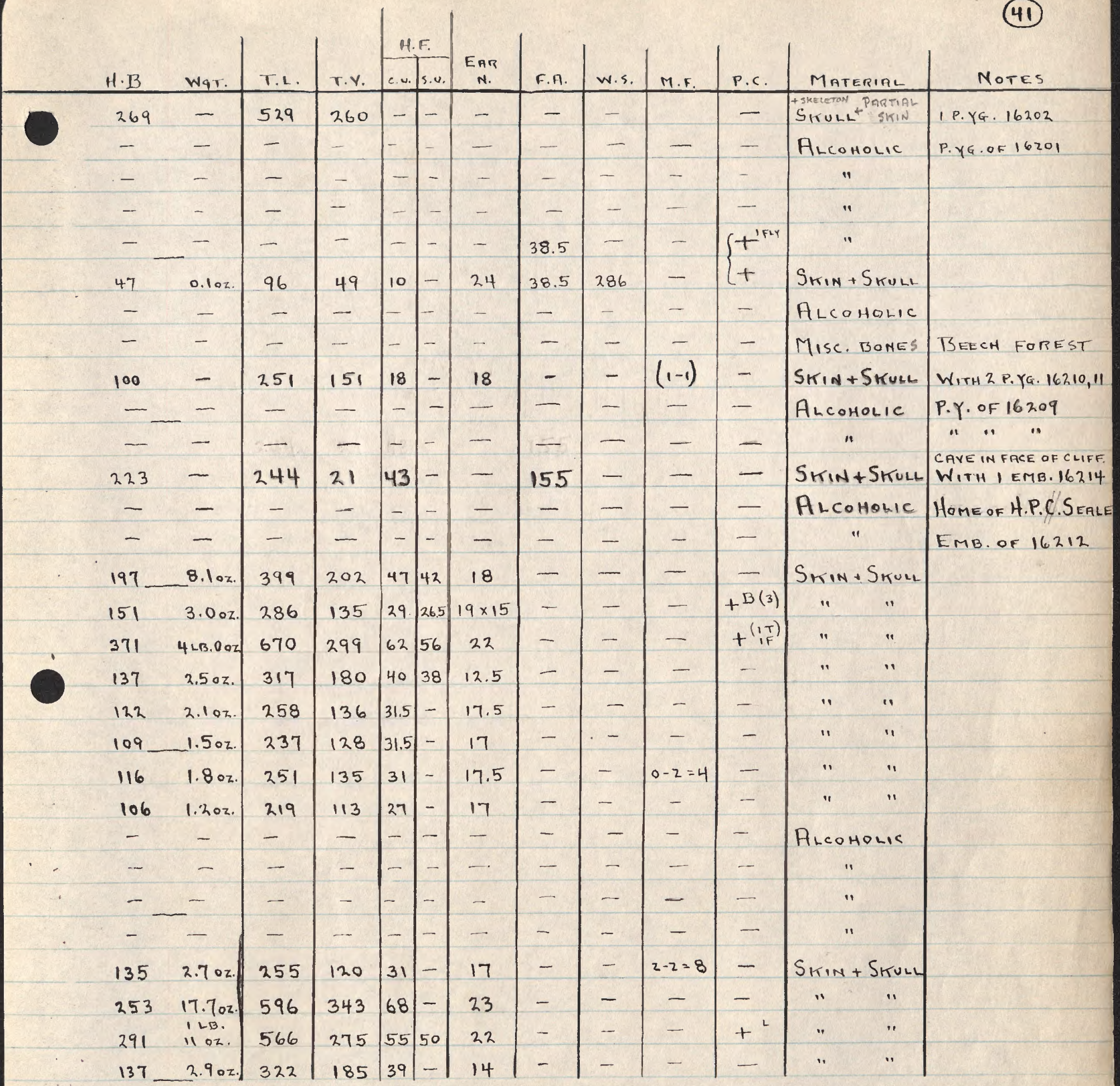




(42)

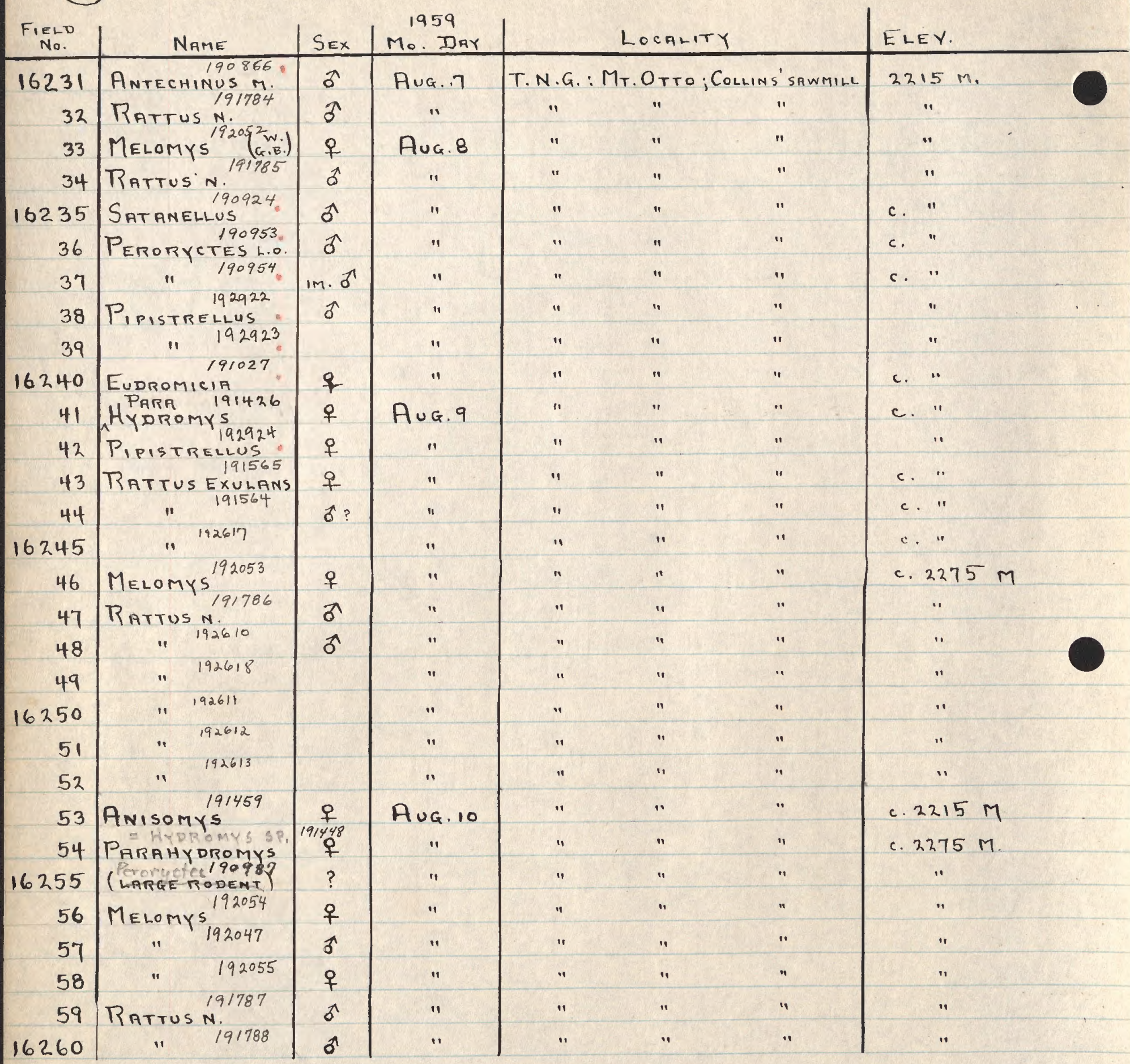

1260 


\begin{tabular}{|c|c|c|c|c|c|c|c|c|c|c|c|c|}
\hline $\mathrm{H} \cdot \mathrm{B}$ & Wat. & T.L. & T.V. & c.u. & 5.u. & $\begin{array}{c}E R T \\
N .\end{array}$ & F.A. & W.S. & $M \cdot F$. & P.C. & MATERIAL & NOTES \\
\hline 113 & $1.30 z$ & 264 & 151 & 25 & 23 & 15 & - & - & - & $+?^{(3 F)}$ & $S_{\text {KIN }}+S_{\text {KULL }}$ & \\
\hline 100 & $1.10 z$ & 214 & 114 & 27.5 & - & 16 & - & - & - & - & $"$ & \\
\hline 113 & $1.80 z$ & 248 & $\begin{array}{l}135 \\
5 L G+T\end{array}$ & 31 & -1 & 18 & - & - & $0-2=4$ & - & $"$ & \\
\hline 114 & $1.70 z$ & 239 & 125 & 28.5 & -1 & 17 & - & - & - & - & $" \quad "$ & \\
\hline 202 & $-5.4 \mathrm{oz}$ & 343 & 191 & 45 & 41.5 & $24 \times 17$ & - & - & - & - & " & \\
\hline 272 & $15.00 z$ & 468 & 196 & 65 & 59 & 26 & - & - & - & - & $" \quad "$ & \\
\hline 131 & $2.00 \mathrm{z}$ & 259 & 128 & 42 & 38.5 & 16 & - & - & - & - & " & \\
\hline - & - & - & - & - & - & - & 37 & - & - & - & ALCOHOLIC & \\
\hline- & - & - & - & - & - & - & 37 & - & - & - & ALCOHOLIC & \\
\hline 97 & $-1.00 z$ & 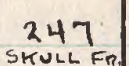 & 150 & 19 & - & 17 & - & - & $(1-1)$ & - & $S_{K I N}+S_{K U L L}$ & \\
\hline$(219)$ & $13.3 \mathrm{oz}$ & $(500)$ & 281 & 59 & 56 & 14 & - & - & $\begin{array}{l}\text { N.F. } \\
0-2=4\end{array}$ & $+{ }^{B}$ & "SKELLETón & \\
\hline - & - & - & - & - & - & - & 36 & - & - & $+{ }^{(2)}$ & AlcoHolic & \\
\hline 113 & 1.7 oz. & 226 & 113 & 24.5 & - & 15.5 & - & - & $2 \cdot 2=8$ & - & SKIN + SKULL & \\
\hline 55 & 0.2502. & 105 & 50 & 16 & - & 7.5 & - & - & - & - & $"$ & \\
\hline - & - & - & - & - & - & - & - & - & - & - & Alcoholic & \\
\hline 107 & $1.60 z$ & 234 & 127 & 31 & - & 18 & - & - & $0-2=4$ & - & S KIN + S KULL & \\
\hline 109 & $1.50 z$ & 221 & 112 & 26.5 & - & 17 & - & - & - & - & $"$ & \\
\hline 一 & - & - & - & - & - & - & - & - & - & - & AlcoHOLIC & \\
\hline - & - & - & - & - & - & - & - & - & - & - & $"$ & \\
\hline - & - & - & - & - & - & - & - & - & - & - & " & \\
\hline - & - & - & - & - & - & - & - & - & - & - & $"$ & \\
\hline - & - & - & - & -1 & - & - & - & - & - & - & $"$ & \\
\hline 251 & 15.802 & 561 & 310 & 68 & 62.5 & 22 & - & - & $1-2=6$ & - & SKIN + SkULL & \\
\hline 131 & 2.502. & 296 & 165 & 36.5 & - & 12.5 & - & - & $0-2=4$ & - & Eskätion " & 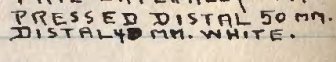 \\
\hline - & - & - & 一 & - & - & - & - & - & - & - & I FRONTFOOT & \\
\hline 122 & 1.902 & 258 & 136 & 31 & - & 18 & - & - & $0-2=4$ & 一 & Sirin + SHULL & \\
\hline 119 & $1.90 z$ & 262 & 143 & 32.5 & - & 18 & - & - & - & - & $"$ & \\
\hline 103 & $1.30 z$ & 227 & 124 & 29.5 & - & 17 & - & - & $0-2=4$ & - & & \\
\hline 113 & $1.7 \mathrm{ez}$. & 232 & 119 & 29.5 & - & 17.5 & - & - & - & - & $"$ & \\
\hline 103 & $1.10 z .1$ & 218 & 115 & 28.5 & - & $\mid 6.5$ & - & - & & - & $"$ & \\
\hline
\end{tabular}




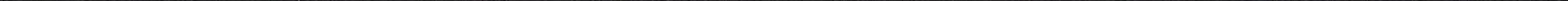


0

○

- 
(43)

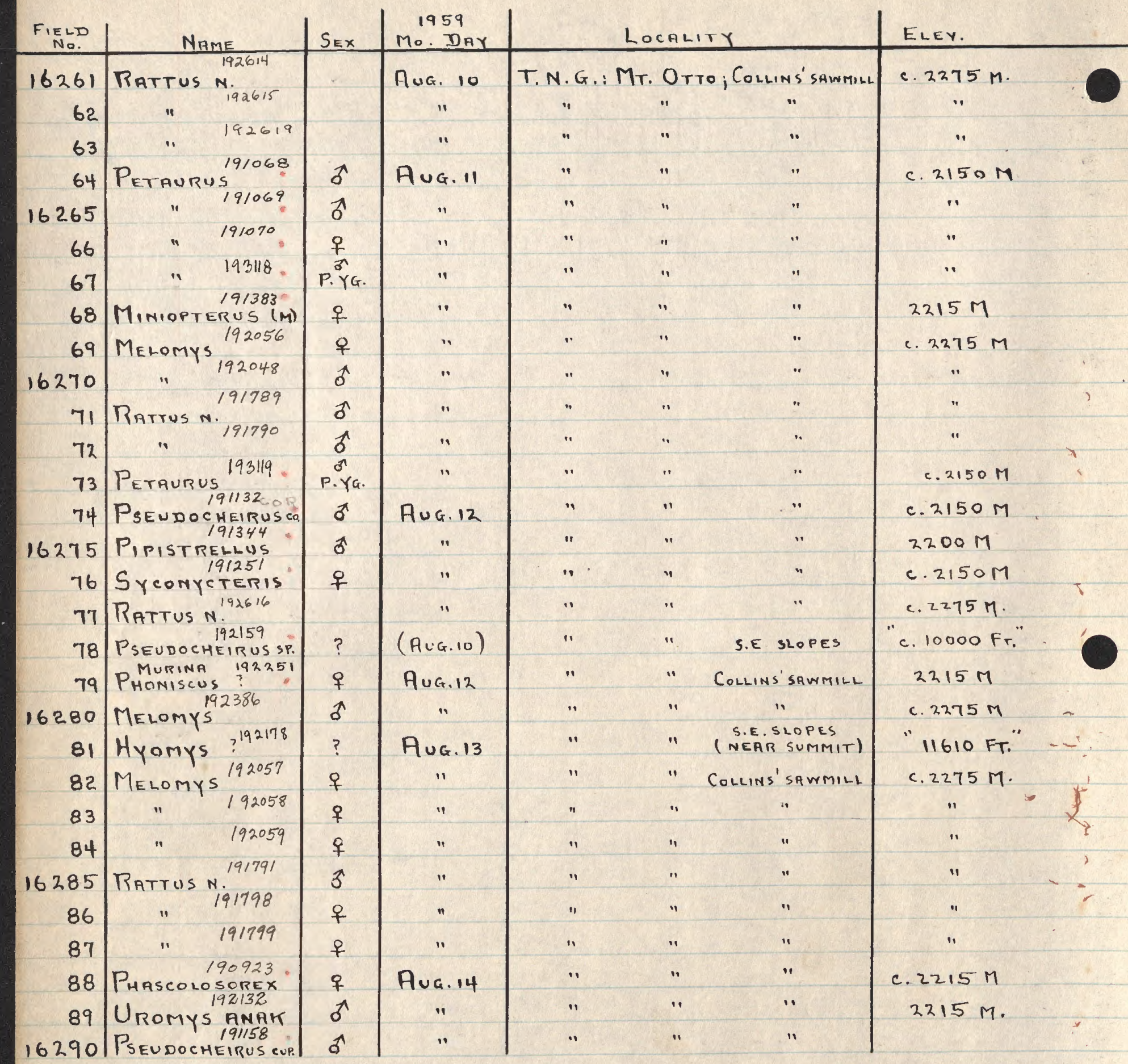

\# 16279 MY bIG EXCITEMENT OF THE DAY CAME WHEN THE YOUNG CHIMBU, WHO TAKES CARE OF THE PIGS, CAME UP WITH A SMALL BBT WHICH HE FOUND IN A CREYICE BETWEEN THE WODDEN CEILING AND TIN ROOF OF THE "BOY HOUSE". IT HAS THE TRICOLOR FUR of PhONISCUS But NOT THE GOLDEN HAIRS. NOSTRILS OPEN LATERALLY FROM LITTLE tubes on nOse; Wing membranes pale brown. This could be Murina - not knOWN FROM NEW GUINER, 


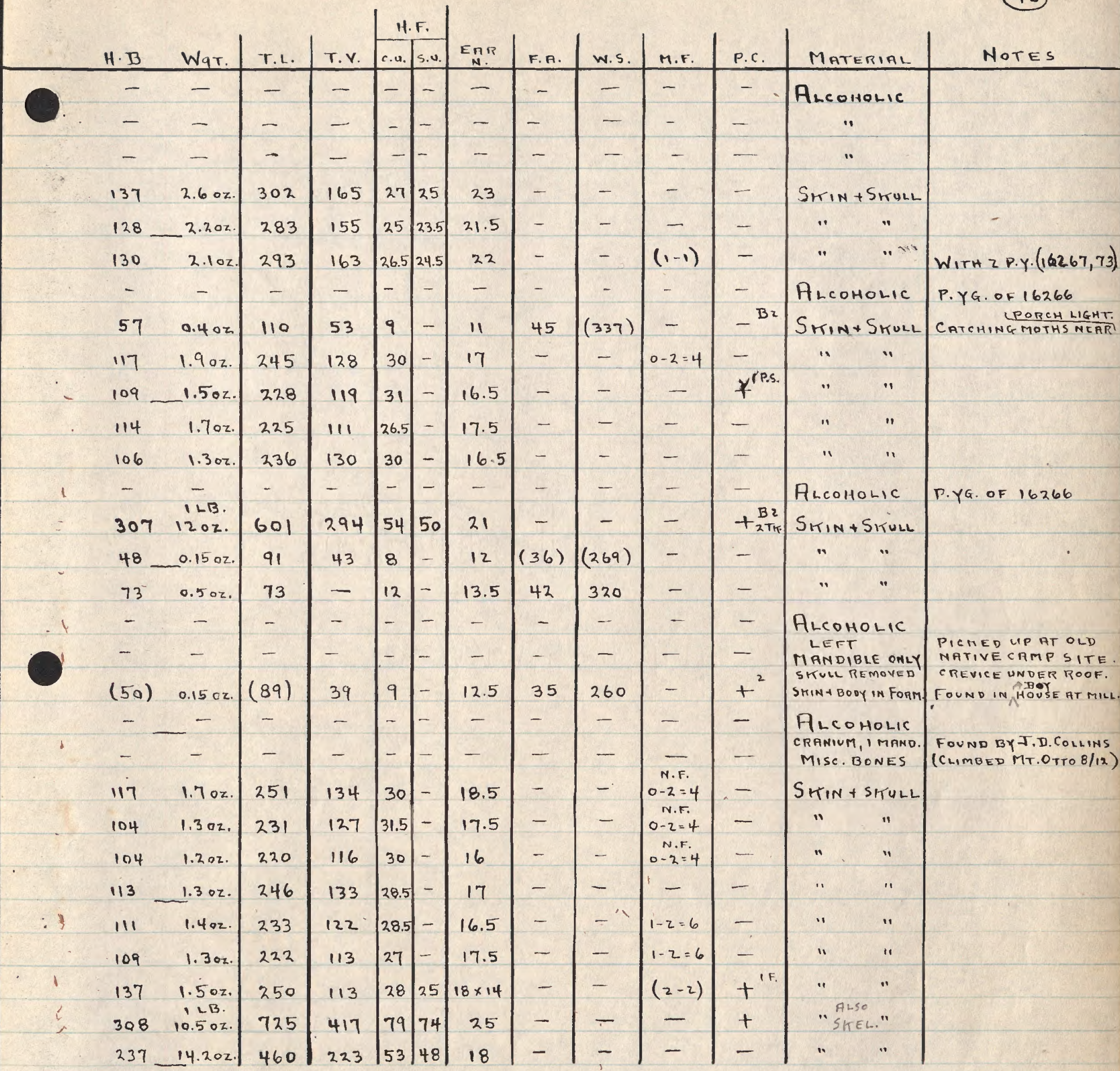



0

-

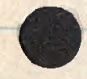




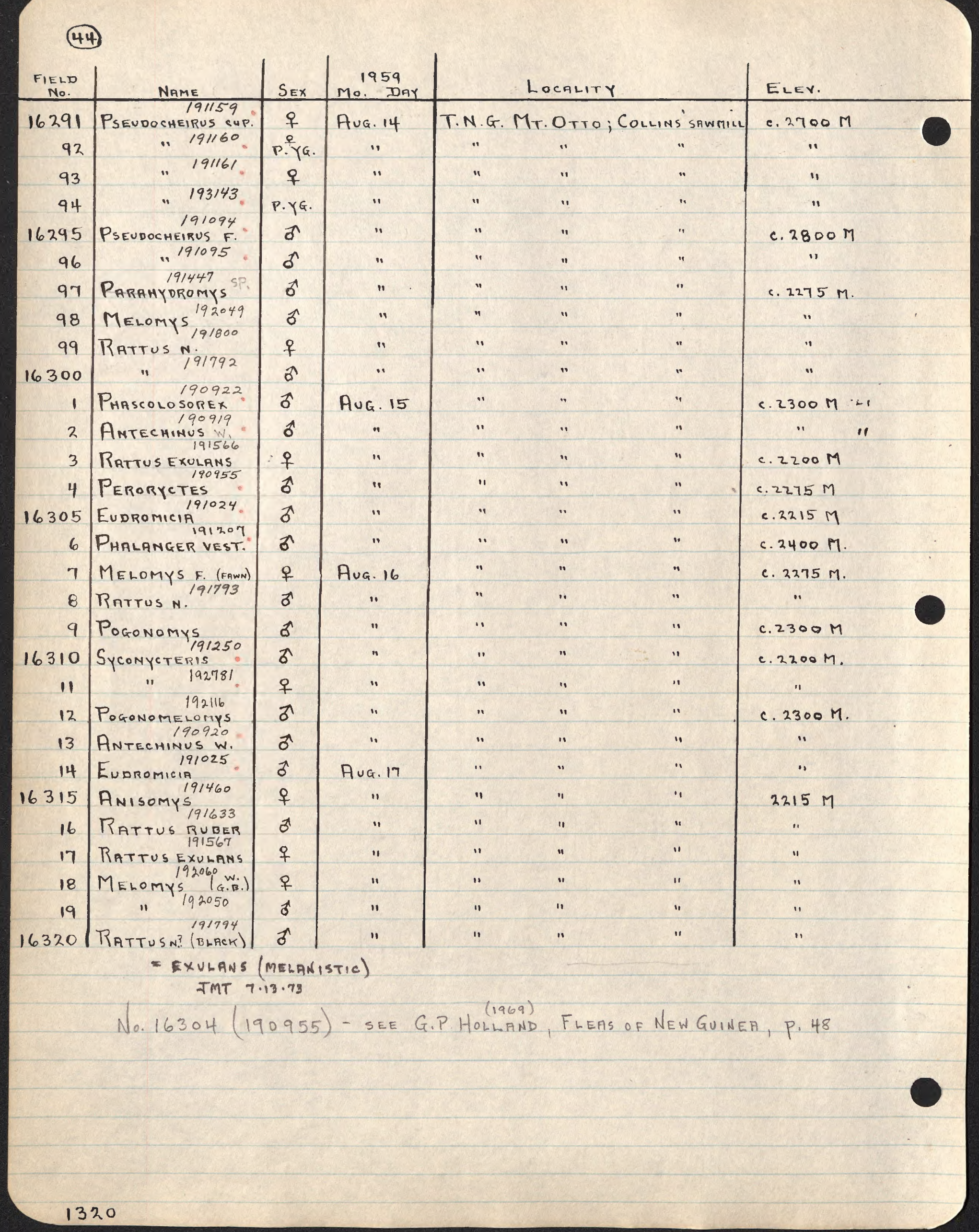




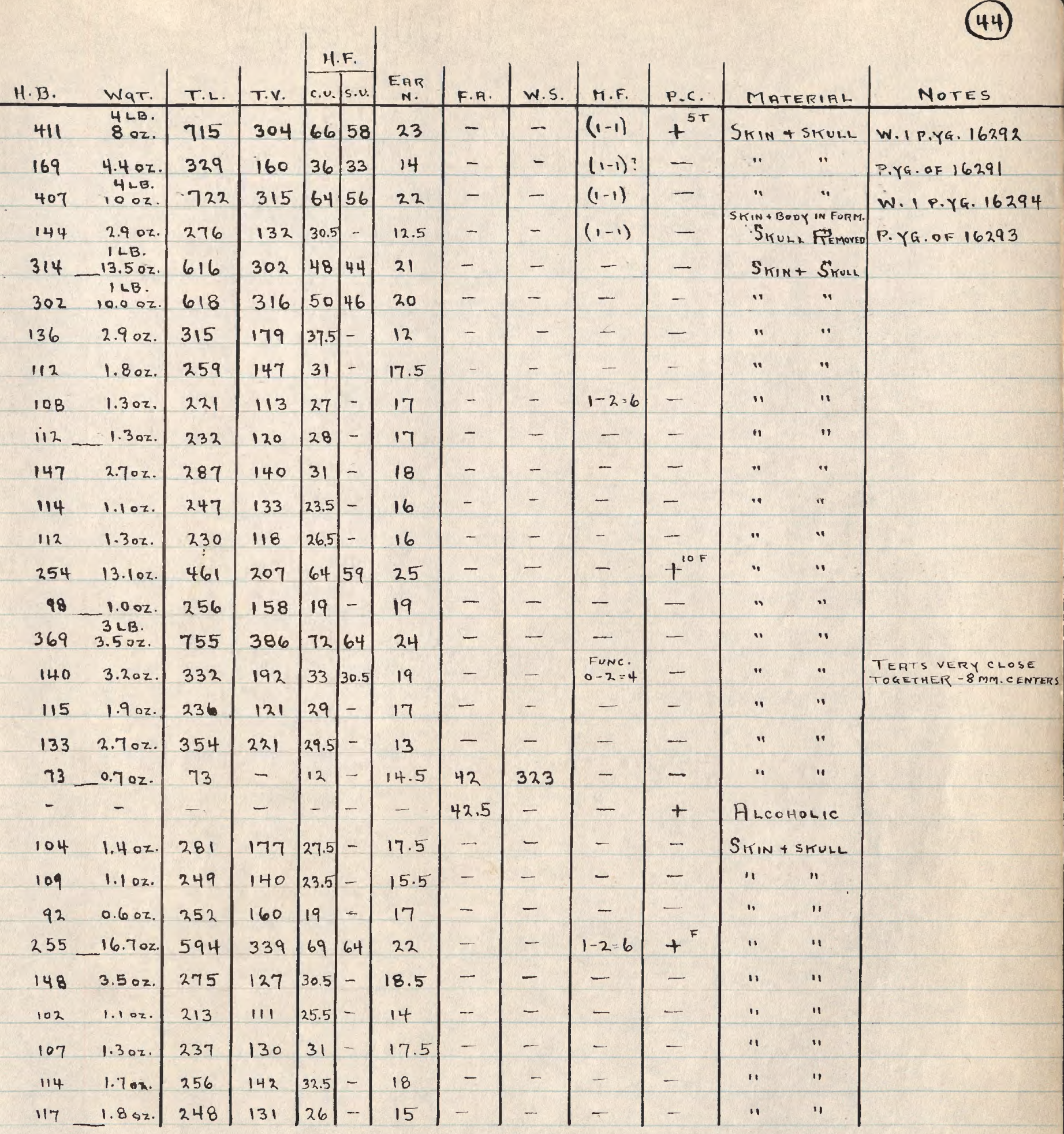




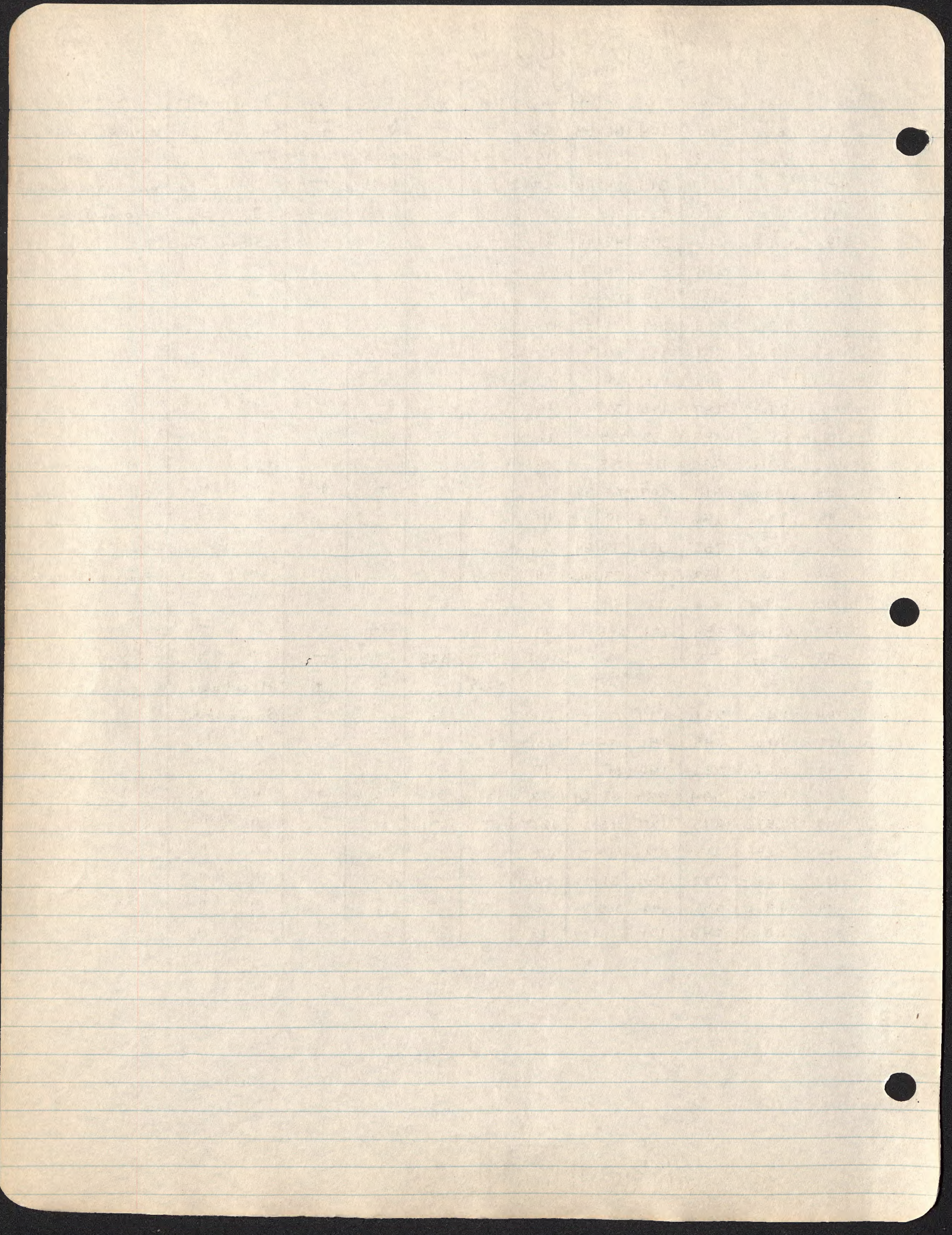


0

-

D

○ 
(45)

\begin{tabular}{|c|c|c|c|c|c|c|c|}
\hline $\begin{array}{l}\text { FIELD } \\
\text { NO. }\end{array}$ & NAME & SEX & Mo. ${ }^{1959}$ DAY & & ELITY & & ELEVATIOH \\
\hline 16321 & Rattusn. 191801 & 우 & Auc. 17 & T.N.G: MT: & DTT & ; Colzons' sammill & $2215 \mathrm{M}$ \\
\hline 22 & $" 192620$ & & $"$ & $"$ & $"$ & $"$ & . \\
\hline 23 & " 192621 & & $"$ & $"$ & $"$ & $"$ & $"$ \\
\hline 24 & " 192622 & & $"$ & $"$ & $"$ & $"$ & " \\
\hline 16325 & 11 192623 & & $"$ & $"$ & $"$ & " & ". \\
\hline 26 & EUDROMICIA & ऊ & $"$ & $"$ & $"$ & $"$ & c. $2300 \mathrm{M}$ \\
\hline 27 & $\begin{array}{r}191028 \\
191029\end{array}$ & q & $"$ & $"$ & " & " & " \\
\hline 28 & $11 \quad 191029$ & q & Aug.18 & $"$ & $"$ & $"$ & $2200 \mathrm{M}$ \\
\hline 29 & $\begin{array}{r}191568 \\
\text { RRTTUSEXULANS }\end{array}$ & 우 & $"$ & * & $"$ & 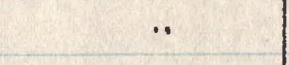 & $2215 \mathrm{M}$ \\
\hline 16330 & Rattus NIOBE & $\widehat{0}$ & $"$ & " & . & $"$ & " \\
\hline 31 & $\begin{array}{l}191796 \\
191384\end{array}$ & 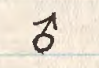 & $"$ & $"$ & " & " & $"$ \\
\hline 32 & $\begin{array}{l}19 / 384 \\
\text { MINIOPTERUS }\end{array}$ & q & Aug. 19 & $"$ & $"$ & OMAHA"GI CRVIE & c. $2275 \mathrm{M}$. \\
\hline 33 & 11191385 & q & $"$ & $"$ & $"$ & $" \quad "$ & ". \\
\hline 34 & "192995. & f & " & $"$ & " & $"$ & " \\
\hline 16335 & "192996 & ㅇ & " & $"$ & $"$ & " $"$ & " \\
\hline 36 & " 192997 & ㅇ & " & $"$ & $"$ & " " & " \\
\hline 37 & " 192998 & 오 & $"$ & " & $"$ & " & " \\
\hline 38 & PERORYCTES Lio & 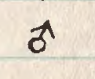 & " & $"$ & $"$ & $"$ & $2215 \mathrm{M}$ \\
\hline 39 & RatTuSN. & f & $"$ & " & " & $"$ & $"$ \\
\hline 16340 & $\begin{array}{l}190867 \\
\text { ANTECHINUS M: }\end{array}$ & $\sigma$ & $"$ & " 18 & $"$ & (1) & c. $2200 \mathrm{M}$ \\
\hline 41 & $\begin{array}{l}191635 \\
\text { RATTUS RUEER }\end{array}$ & $\widehat{d}$ & AUG.21 & T.N.G.: BEN & 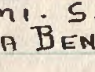 & $\begin{array}{l}\text { OF GOROKA) } \\
\text { IA PLANTATIRN }\end{array}$ & c. $4700 F_{T}$. \\
\hline 42 & TRATUS EXULANS & $\hat{\sigma}$ & $"$ & $"$ & & $"$ & " \\
\hline 43 & " 191570 & $\hat{d}$ & $"$ & " & & $"$ & $"$ \\
\hline 44 & 1191573 & $q$ & $"$ & $"$ & & $"$ & $"$ \\
\hline 16345 & 191574 & f & $"$ & $"$ & & $"$ & " \\
\hline 46 & " 191575 & $q$ & " & $\because$ & & $"$ & $"$ \\
\hline 47 & $\begin{array}{l}\text { 19/211 } \\
\text { RouseTTUS }\end{array}$ & ठ & Aua. 22 & $"$ & & $"$ & " \\
\hline 48 & $\begin{array}{l}191571 \\
\text { Rattus exuLANS }\end{array}$ & $\delta$ & " & $"$ & & $"$ & " \\
\hline 49 & " 191576 & 우 & " & " & & " & $"$ \\
\hline 16350 & 191577 & 우 & " & " & & " & " \\
\hline
\end{tabular}

No. $16338(190956)$ - G.P.HOLLAND $(1969)$, FLEAS OFN.G., P. 48 


\begin{tabular}{|c|c|c|c|c|c|c|c|c|c|c|c|c|}
\hline \multirow[b]{2}{*}{$\mathrm{H} \cdot \mathrm{B}$} & \multirow[b]{2}{*}{ WaT. } & \multirow[b]{2}{*}{ T.L. } & \multirow[b]{2}{*}{ T.V. } & \multicolumn{2}{|c|}{ H.F. } & \multirow[b]{2}{*}{$\begin{array}{l}\text { ERT } \\
N \text {. }\end{array}$} & \multirow[b]{2}{*}{ F.A. } & \multirow[b]{2}{*}{ w.s. } & \multirow[b]{2}{*}{ M.F. } & \multirow[b]{2}{*}{ P.c. } & \multirow[b]{2}{*}{ MATERIAL } & \multirow[b]{2}{*}{ NOTES } \\
\hline & & & & e.u. & s.u. & & & & & & & \\
\hline 109 & $1.3 \mathrm{oz}$. & 217 & 108 & 26.5 & - & 18 & - & - & $1-2=6$ & - & SKIN + SKULL & \\
\hline - & - & - & - & - & - & - & - & - & - & - & Alcoholic & \\
\hline - & - & - & - & - & - & - & - & - & - & - & " & \\
\hline - & - & - & - & - & - & - & - & - & - & - & " & \\
\hline - & - & - & - & - & - & - & - & - & - & - & " & \\
\hline 92 & $0.80 z$ & 251 & 159 & 195 & - & 17 & - & - & - & - & $S_{K I N}+S_{K U L L}$ & \\
\hline 91 & $0.750 z$ & 247 & 156 & 19 & - & 17.5 & - & - & $\left|\begin{array}{c}N-F \\
(z-z)\end{array}\right|$ & $+{ }^{B}$ & $"$ & \\
\hline 91 & $0.80 z$ & 227 & 136 & 18.5 & - & 17 & - & - & & - & " i & $\begin{array}{l}\text { FOUND WW DAYTIME. } \\
\text { IN PANDANUS TREE: }\end{array}$ \\
\hline 123 & $2.00 \mathrm{z}$ & 249 & 126 & 27 & - & 16 & - & - & $2-2=8$ & 一 & $"$ & \\
\hline 114 & $-1.70 z$ & 266 & 152 & 29.5 & - & 17 & - & - & - & - & $" \quad$ & \\
\hline 109 & $1.60 z$ & 233 & 124 & 29 & - & 17.5 & - & - & - & 一 & $" \quad "$ & \\
\hline 60 & $0.30 x$. & 115 & 55 & 9.5 & - & 10.5 & 46 & 345 & - & - & SMIN + SKURL & \\
\hline 57 & 0.302. & 109 & 52 & 9 & - & 10 & 45 & 334 & - & - & " & \\
\hline - & - & - & - & - & - & - & 45.5 & - & - & - & Alcohonic & \\
\hline- & - & - & - & - & - & - & 42.5 & - & - & - & $"$ & \\
\hline - & - & - & - & - & - & - & 46 & - & - & - & $"$ & \\
\hline - & - & - & - & - & - & - & 45 & - & - & - & " & \\
\hline 275 & 14.302 & 481 & 206 & 66 & 61 & 26 & - & - & - & +5 & SKIN + SKULL & \\
\hline 102 & $1.1 \mathrm{oz}$ & 188 & 86 & 27.5 & - & 15 & - & - & - & - & $"$ & \\
\hline 87 & - & 204 & 117 & 22 & - & 15 & - & - & - & - & $"$ & \\
\hline 162 & $4.9 \mathrm{oz}$ & 320 & 158 & 33.5 & - & 18.5 & - & - & - & - & $S_{W I H}+5 K U L L$ & \\
\hline 121 & $2.00 z$ & 253 & 132 & 25.5 & - & 16 & - & - & - & - & $"$ & \\
\hline 117 & $1.90 z$ & 248 & 131 & 26.5 & - & 15.5 & - & - & - & - & $"$ & \\
\hline 114 & $1.50 z$ & 249 & 135 & 26 & - & 17 & - & - & - & - & $"$ & - \\
\hline 122 & $-1.9 \mathrm{cz}$. & 245 & 123 & 24.5 & - & 16 & - & - & $2-2=8$ & - & $"$ & \\
\hline 112 & $1.30 x$ & 243 & 131 & 26.5 & - & 16.5 & - & - & - & - & $"$ & \\
\hline 135 & $3.8 \mathrm{or}$ & 151 & 16 & 21 & - & 19 & 86.5 & 575 & - & - & 11 & NETTED UNDER FienS \\
\hline 127 & $2.00 x$ & 275 & 148 & 27 & - & 16.5 & - & - & - & - & " & \\
\hline 122 & $2.0 \mathrm{oz}$ & 248 & 126 & 24.5 & - & 17 & - & - & $2-2=8$ & - & " & \\
\hline 105 & $1.20 z$. & 225 & 120 & 25.5 & -1 & 15.5 & - & - & - & - & $"$ & \\
\hline
\end{tabular}




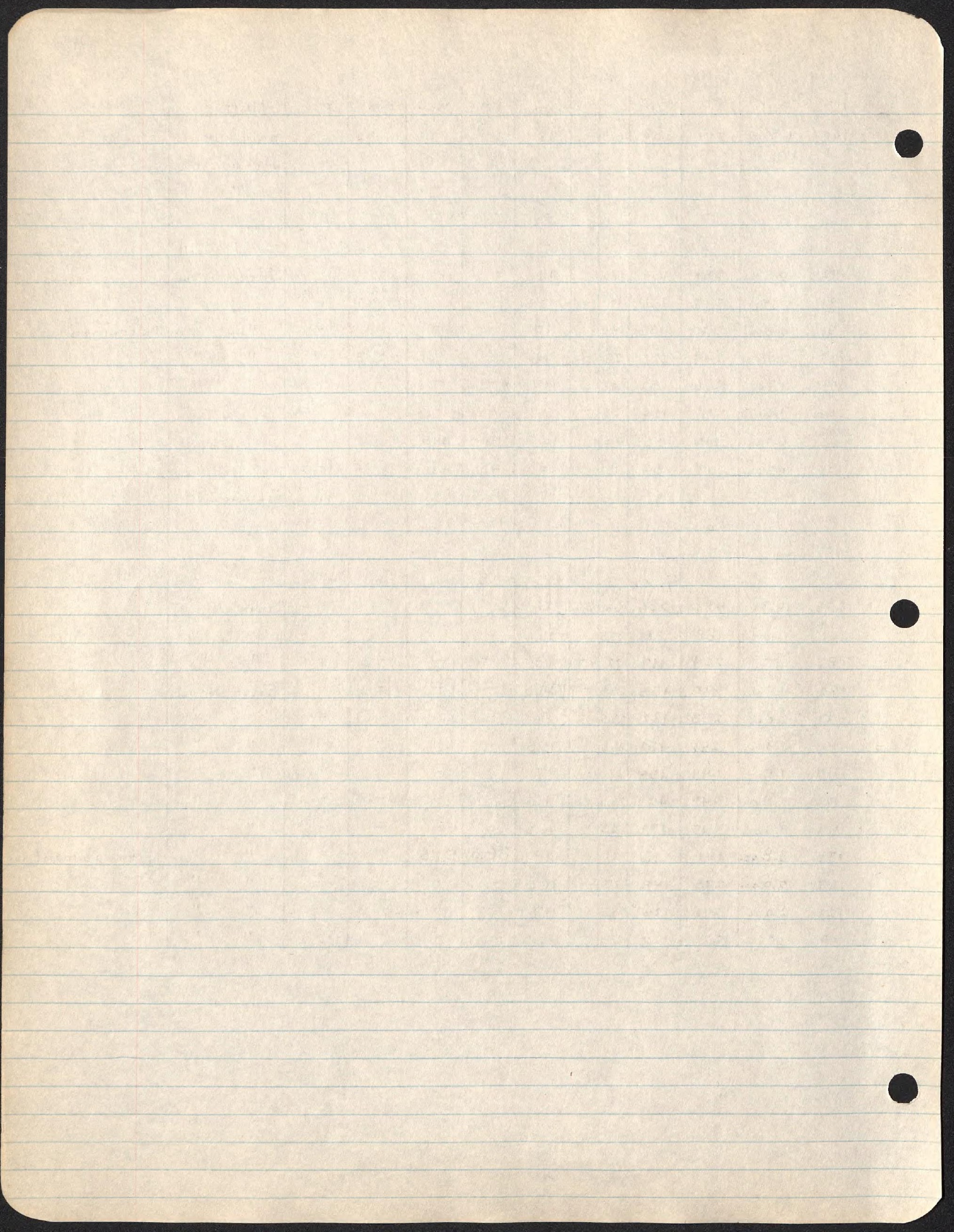


-

○

○ 
(46)

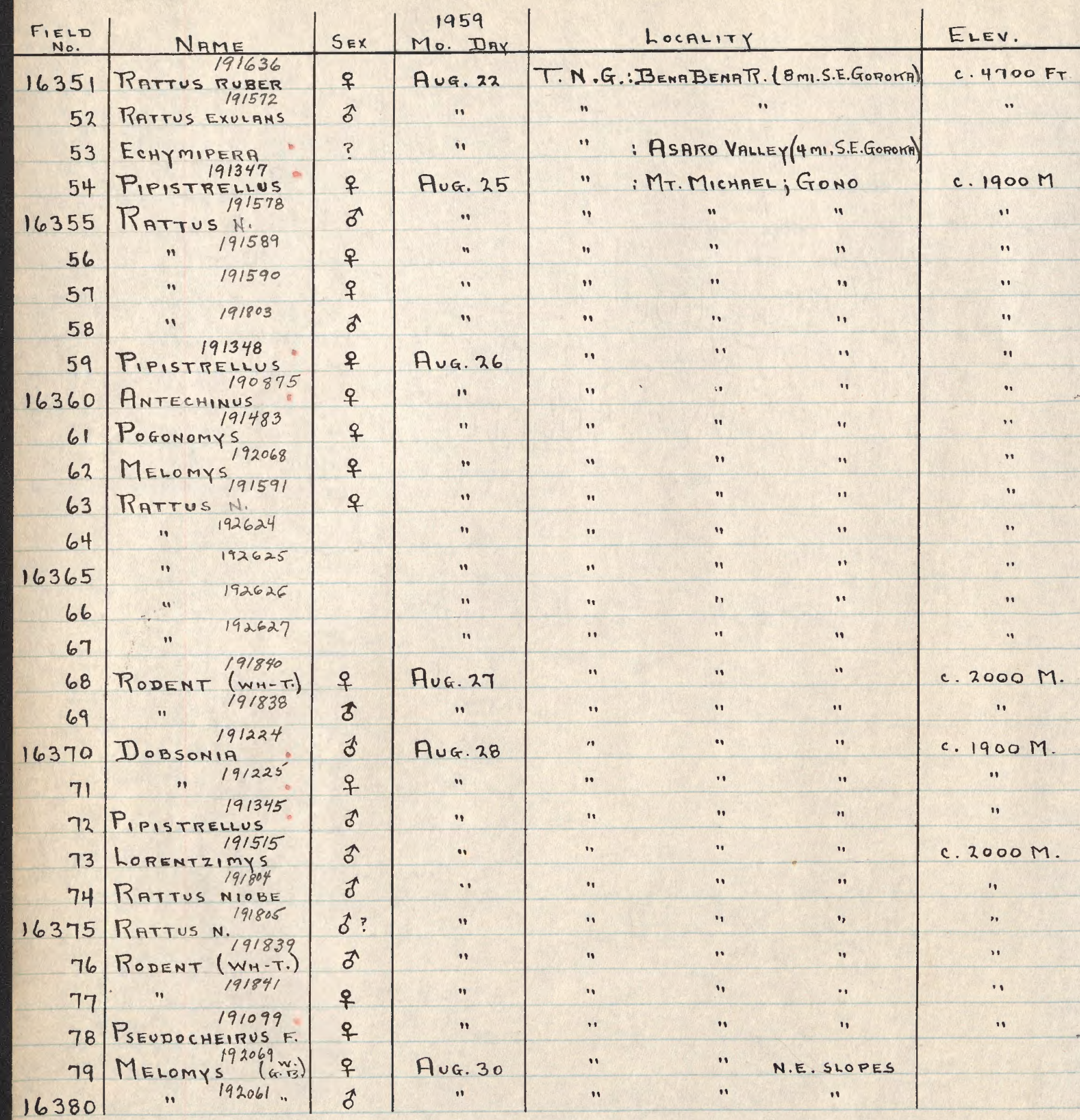




\begin{tabular}{|c|c|c|c|c|c|c|c|c|c|c|c|c|c|}
\hline$H \cdot B$ & War. & $T . L$. & T.V. & c.u. & s.u. & $\begin{array}{l}\text { EAR } \\
N \text {. }\end{array}$ & F.A. & w.s. & M.F. & P.C. & \multicolumn{2}{|c|}{ MATERIAL } & NOTES \\
\hline 141 & $2.80 z$ & 268 & 127 & 29 & - & 18.5 & - & - & $2-2=8$ & - & \multicolumn{2}{|c|}{$S_{K I N}+S_{K U L L}$} & \\
\hline 82 & $0.60 z$ & 175 & 93 & 23.5 & - & 12.5 & - & - & - & - & " & & \\
\hline - & - & - & - & - & - & 21.5 & - & - & - & - & \multicolumn{2}{|c|}{ SITULL ONLY } & GRASSLAND \\
\hline 51 & $0.250 z$ & 89 & 38 & 10 & - & 11 & 37 & 269 & - & - & \multicolumn{2}{|c|}{ SKIN+SKUHL } & \\
\hline 119 & $1.90 z$ & 246 & 127 & 26 & - & 16 & - & - & - & - & $\because$ & " & \\
\hline 115 & $1.7 a z$ & 235 & 120 & 26 & - & 16 & - & - & $2-2=8$ & - & $"$ & $\because$ & \\
\hline 95 & $1.00 z$ & 197 & 102 & 24 & - & 15 & - & - & - & - & $\because$ & $\because$ & \\
\hline 87 & $0.70 z$ & 165 & 78 & 25.5 & - & 16 & - & - & - & - & $\because$ & $"$ & \\
\hline 50 & 0.15 oz. & 94 & 44 & 10 & - & 12 & $(36+)$ & $(279)$ & - & +1 & $"$ & $"$ & \\
\hline 118 & $1.5 \mathrm{oz}$ & 271 & 153 & 25.5 & - & $17 \times 13$ & - & - & $(2-2)$ & - & " & $"$ & \\
\hline 136 & $2.70 z$. & 351 & 215 & 31 & - & 15 & - & - & $1-2=6$ & - & $"$ & $"$ & \\
\hline 125 & $2.0 \mathrm{oz}$ & $(213)$ & $\begin{array}{l}5 T \cup B \\
(88)\end{array}$ & 31 & - & 17 & - & - & $0-2=4$ & - & $\cdots$ & $" 1$ & \\
\hline 114 & $1.7 \mathrm{oz}$ & 228 & 114 & 25 & - & 15 & - & - & - & - & 11 & $"$ & \\
\hline$\rightarrow$ & - & 一 & - & - & - & - & - & - & - & $\cdot-$ & \multicolumn{2}{|c|}{ AlCOHOLIC } & \\
\hline - & - & - & - & - & - & - & - & - & - & - & \multicolumn{2}{|c|}{$"$} & \\
\hline - & - & - & - & - & - & - & - & - & - & - & \multicolumn{2}{|c|}{$"$} & \\
\hline - & - & - & - & - & - & - & - & - & - & - & \multicolumn{2}{|c|}{$"$} & \\
\hline 116 & $1.60 \mathrm{z}$. & 268 & 152 & 34 & - & 18.5 & - & - & ? & $\left\{+t^{15-2}\right.$ & \multicolumn{2}{|c|}{ SKIN + SKULL } & $\begin{array}{l}10 \text { FOREST. DISTAL } \\
45-48 \mathrm{mM} \text {. T AIL WHITE. }\end{array}$ \\
\hline 117 & $1.7 \mathrm{oz}$. & 273 & 156 & 34 & - & 18 & - & - & 一 & $(+)$ & $"$ & $"$ & $\begin{array}{l}\text { FEET + WRISTS + NAILS } \\
\text { WWITE. }\end{array}$ \\
\hline 248 & $14.30 \mathrm{z}$ & 278 & 30 & 46 & - & 32.5 & 152 & - & - & - & $"$ & $"$ & \\
\hline 225 & & 257 & 32 & 45 & - & 31 & 152 & - & $1-0=2$ & - & $"$ & $"$ & \\
\hline - & - & - & 40 & 10 & - & 11.5 & 36.5 & 273 & 一 & +4 & " & " & \\
\hline 74 & $0.50 z$ & $(199)$ & $\begin{array}{l}\text { TRIL FR. } \\
(125)\end{array}$ & 25 & - & 17.5 & - & - & - & - & $\because$ & $"$ & \\
\hline 111 & $1.40 z$ & 235 & 124 & 29.5 & - & 18 & 一 & - & - & - & $\because$ & $\bullet$ & \\
\hline 80 & $0.70 z$ & 168 & 88 & 22.5 & - & 14 & - & - & - & - & $\ddot{~}$ & $"$ & \\
\hline 140 & $3.0 \mathrm{oz}$ & 328 & 188 & 36 & - & 20.5 & - & - & - & $t^{F+}$ & " & " & \\
\hline 92 & $0.70 z$. & 201 & 109 & 29 & - & 27 & - & - & - & $+B_{2}$ & $\because$ & $"$ & \\
\hline 146 & $2.9 \mathrm{oz}$ & 333 & 187 & 30 & 28 & 17 & - & - & $(1-1)$ & - & . & " & \\
\hline 110 & $1.40 z$ & 237 & 127 & 32 & - & 17 & - & $\rightarrow$ & $0-2=4$ & - & $" 1$ & $\ddot{\prime}$ & \\
\hline 114 & $1.80 z$. & 250 & 136 & $\mid 31$ & $1-$ & 17 & - & - & - & - & 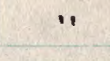 & $" 1$ & \\
\hline
\end{tabular}


○

-

○ 


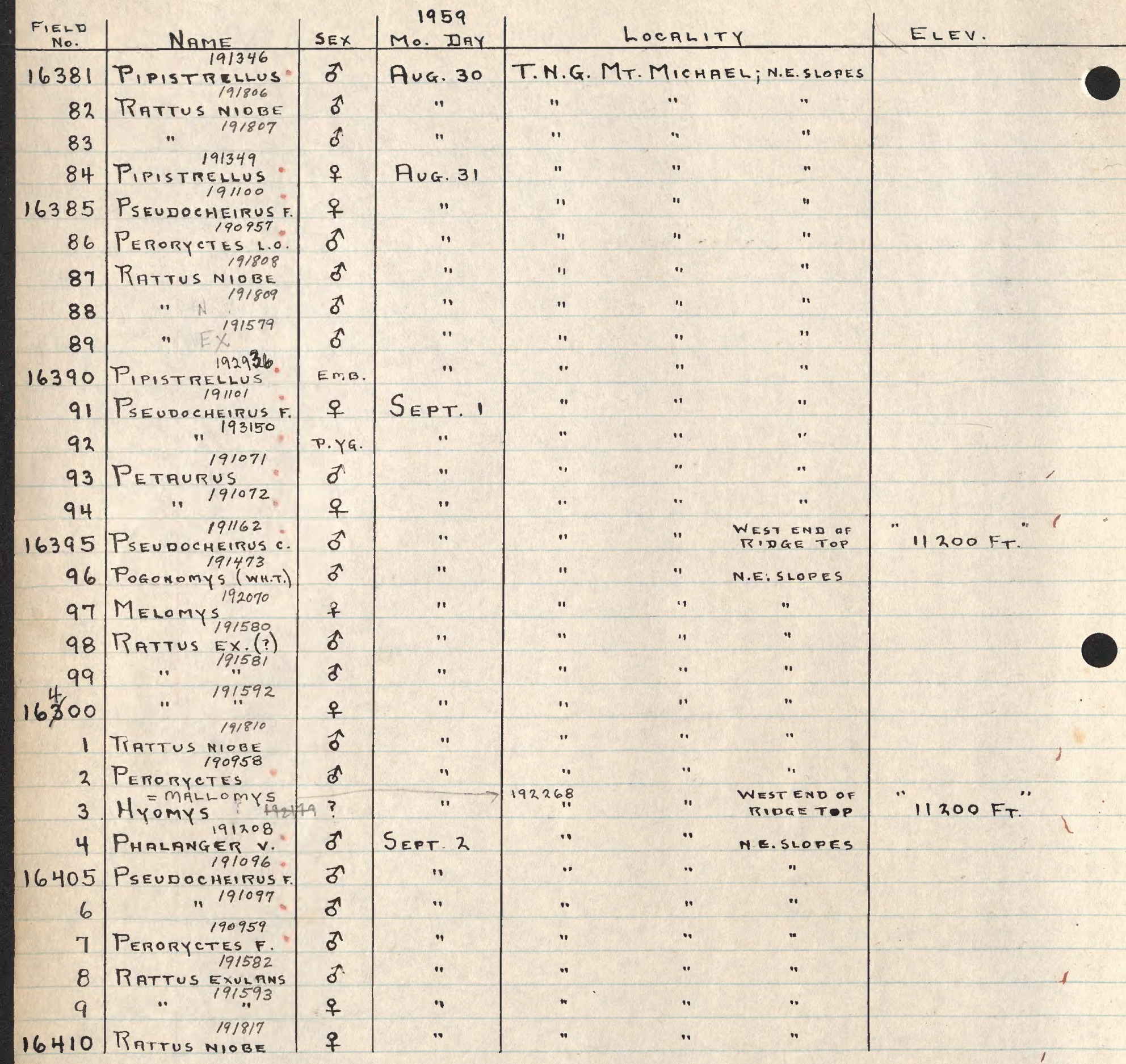




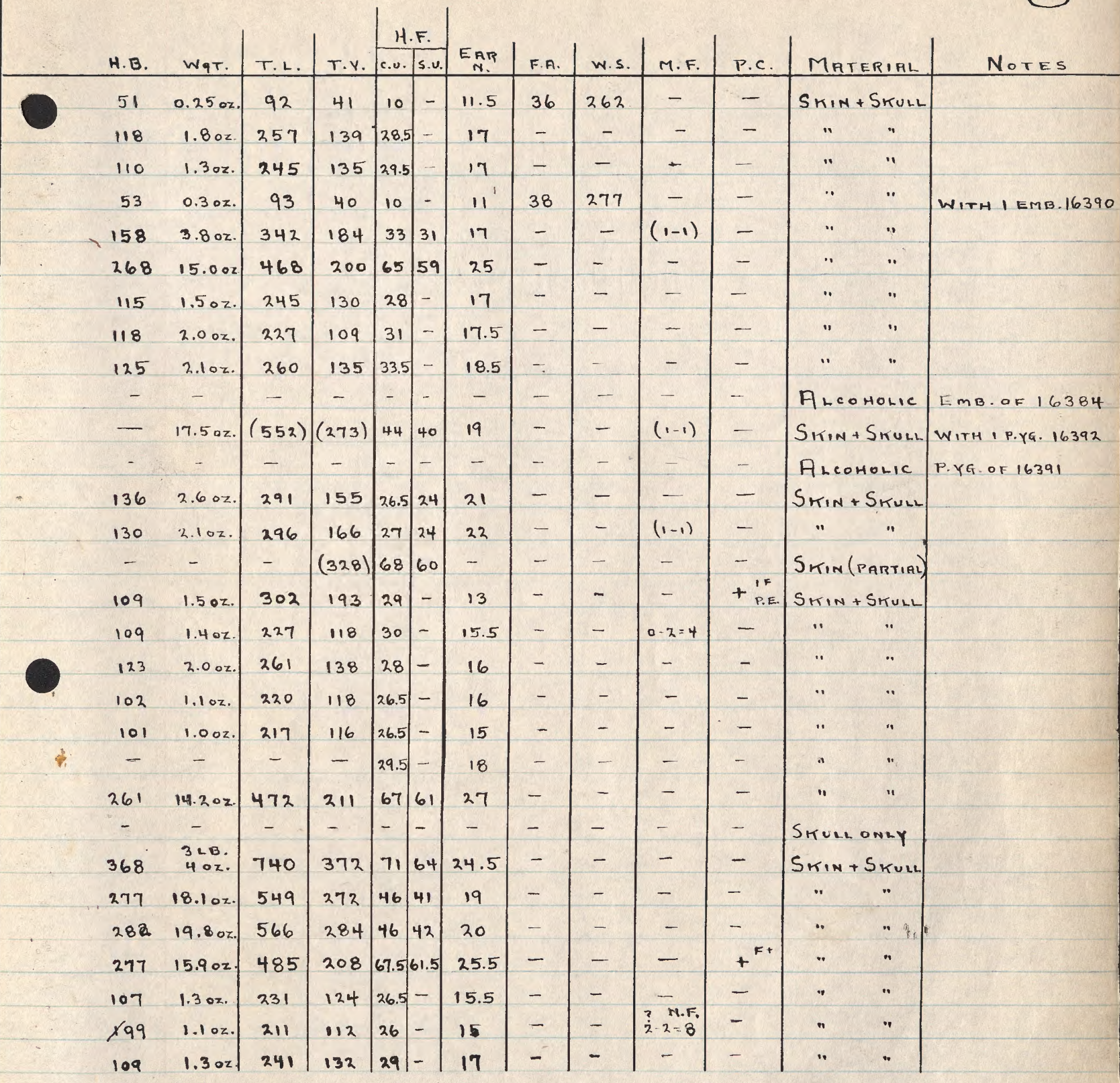



(48)

\begin{tabular}{|c|c|c|c|c|c|c|c|}
\hline $\begin{array}{l}\text { FIELD } \\
\text { NO. }\end{array}$ & NAME & SEX & $\begin{array}{c}1959 \\
\text { Mo. D D }\end{array}$ & \multicolumn{3}{|c|}{ LOCALITY } & ELEVATION \\
\hline 16411 & $\begin{array}{l}191030 \\
\text { EuDROMICIA }\end{array}$ & ㅇ & SEPT. 2 & \multicolumn{3}{|c|}{ T.N.G.MT. MichAEL; LUFA } & \\
\hline 12 & $\begin{array}{l}192151 \\
\text { DENDROLAGUS?" }\end{array}$ & $?$ & $($ (") & " & $"$ & & \\
\hline 13 & RATTUS RUBER & $\hat{\sigma}$ & SEPT. 3 & $"$ & $"$ & ; M.E.SLOPES & \\
\hline 14 & PERORYCTES 190960. & 오 & $"$ & " & ". & $\because$ & \\
\hline 16415 & "193122. & & " & " & $"$ & $"$ & \\
\hline 16 & WALLABY 192152 & $?$ & $"$ & $"$ & $\because$ & & \\
\hline 17 & PSEUDOCHEIRUS F. & $\widehat{\sigma}$ & $S_{\text {EPT. }} 4$ & $"$ & $"$ & N.E. SLOPES & \\
\hline 18 & Melomys 192071 & q & " & $"$ & " & " & \\
\hline 19 & 11 $\quad 192062$ & $\sigma$ & " & “ & " & $"$ & \\
\hline 16420 & Tattus N $^{1918 / 8}$ & $q$ & $"$ & $"$ & $"$ & $"$ & \\
\hline 21 & $111^{19 / 811}$ & $\hat{o}$ & $"$ & $"$ & $"$ & " & \\
\hline 22 & Poganomys 192117 & $\delta$ & " & $"$ & " & " & \\
\hline 23 & MELOMYS 192072 & $q$ & 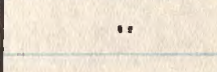 & $"$ & $\because$ & $"$ & \\
\hline 24 & RATTUS N. & & SEPT. $5-6$ & $"$ & $"$ & $"$ & \\
\hline 16425 & $11 \quad 191825$ & & ." & $"$ & $"$ & $"$ & \\
\hline 26 & 11 191826 & & $"$ & $"$ & " & " & \\
\hline 27 & MELOMYS 192074 & ๙ & " & " & $"$ & " & \\
\hline 28 & SYCONYCTERIS & & $"$ & $"$ & $"$ & $"$ & \\
\hline 29 & " 191259 & & $"$ & $\because$ & $"$ & $"$ & \\
\hline 16430 & $119 / 260$ & & $"$ & $"$ & $"$ & $"$ & \\
\hline 31 & 1191261 . & & $"$ & " & $"$ & $"$ & \\
\hline 32 & MELOMYS 192063 & $\hat{\sigma}$ & SEPT. 6 & $"$ & $"$ & RIDGE CAMP & $10200 \mathrm{FT}$. \\
\hline 33 & 11 192073 & 우 & $"$ & " & $"$ & $"$ & $" 1$ \\
\hline 34 & Rattus 192628 & & $"$ & $"$ & $"$ & " & $"$ \\
\hline 16435 & $\begin{array}{rr}1192629 \\
192630\end{array}$ & & " & $"$ & $"$ & $"$ & $"$ \\
\hline 36 & 192629 & & " & $"$ & $"$ & $"$ & $"$ \\
\hline 37 & $\begin{array}{l}192624 \\
192630\end{array}$ & & " & $"$ & $"$ & $" 1$ & " \\
\hline 38 & $\begin{array}{l}192630 \\
192631\end{array}$ & & 11 & $"$ & $"$ & $"$ & $"$ \\
\hline 39 & $" \begin{array}{ll}192631 \\
192632\end{array}$ & & $"$ & $"$ & " & $"$ & $\because$ \\
\hline 164401 & 11 & & $" 1$ & $"$ & $"$ & $"$ & " \\
\hline
\end{tabular}

16411,412 : GIFT - PaUL + Barbara SELLERS, FaIth Mission, Lufa

16424-16431: TAKEN BY LIKLIK IN CAMP ON SEPT. 5 AND 6 WHILE I WAS CLIMBING MT.MICHAEL. L.L. DID MOT SEX OR MERSURE. 
(48)

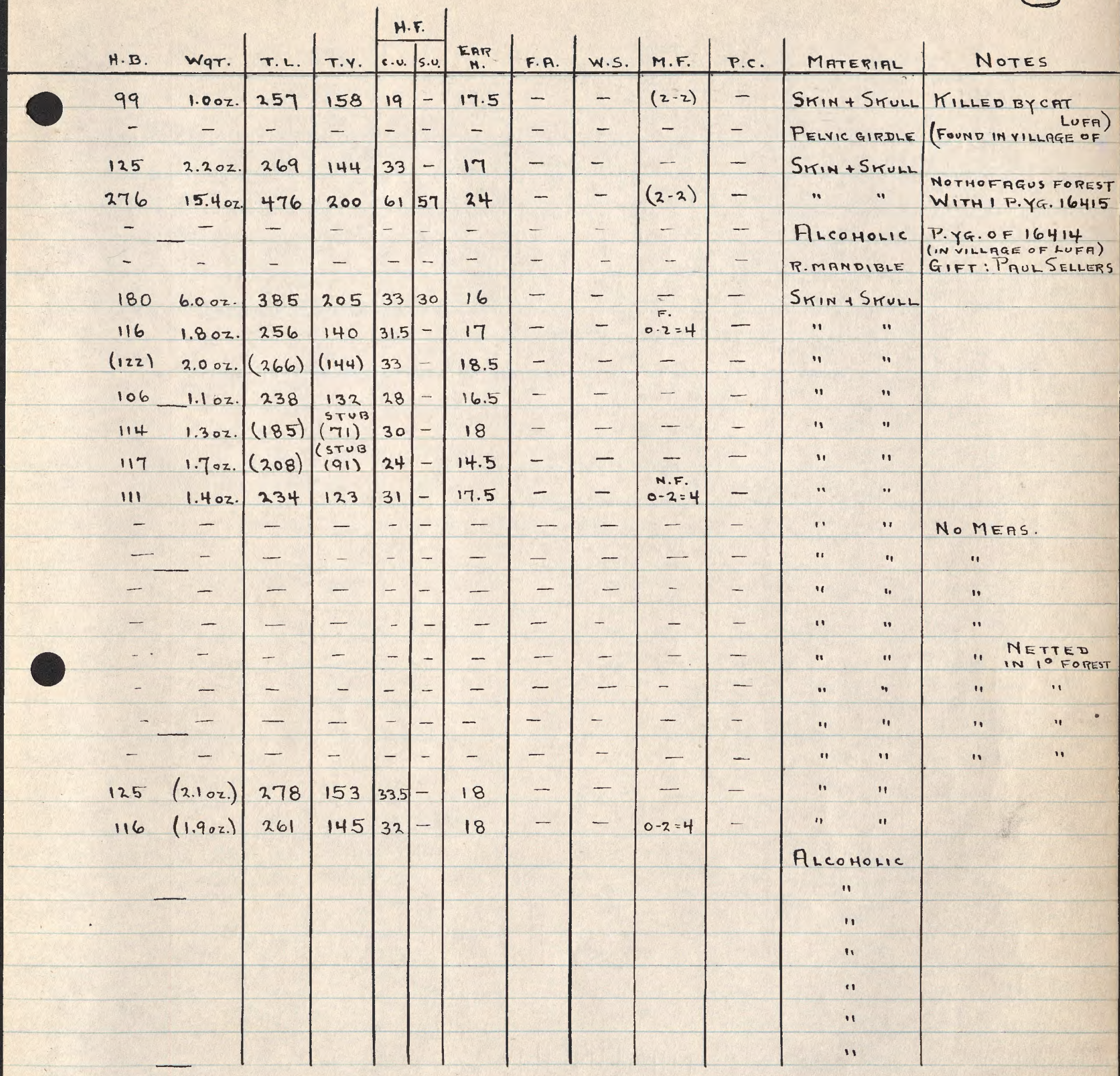




(49)

\begin{tabular}{|c|c|c|c|c|c|c|c|}
\hline $\begin{array}{l}\text { FIELD } \\
\text { No. }\end{array}$ & NAME & SEx & $\begin{array}{l}1959 \\
\text { Mo. } D_{\text {AYY }}\end{array}$ & \multicolumn{3}{|c|}{ LOCALITY } & ELEY. \\
\hline 16441 & RATTUS & & SEPT. 6 & $T \cdot N \cdot G$. & Mt. Mic & HAEL; CAMP & $10,200 \mathrm{FT}$. \\
\hline 42 & PSEUDOCHEIRUS & & $"$ & $"$ & $"$ & SUMMIT RIDGE & c. $11,900 \mathrm{FT}$. \\
\hline 43 & Rattus Nigia & & $"$ & " & $"$ & " & " \\
\hline 44 & 191819 & 운 & SEPT. 7 & $"$ & $"$ & N.E.SLOPES & c. $6500 \mathrm{Fr}$. \\
\hline 16445 & "1 191812 & $\widehat{\sigma}$ & 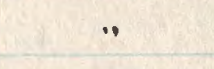 & $"$ & " & " & " \\
\hline 46 & Rattus 192636 ? & q & SEPT. 8 & $"$ & $"$ & $"$ & $"$ \\
\hline 47 & Po MELOMYS (SDY) & 우 & $"$ & $"$ & " & HAGÄVE CREEK & c. $6000 \mathrm{FT}$. \\
\hline 48 & HYDROMYs & 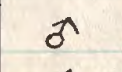 & " & $"$ & $"$ & " " " & " \\
\hline 49 & MELOMYS $\left(\mathrm{G}^{\mathrm{w}} \mathrm{B}\right)$ & $\pi$ & SEPT. 9 & " & $"$ & N.E. SLOPES & c. $6500 F_{T}$. \\
\hline 16450 & Rattus Ex. & $\delta$ & " & $"$ & $"$ & Hagave CreEk & c. $6000 F_{T}$. \\
\hline 51 & ". 191584 & ब & " & $"$ & $"$ & " & " \\
\hline 52 & 191585 & $\sigma$ & " & $"$ & $"$ & $"$ & $"$ \\
\hline 53 & "1 191586 & ๘ & " & " & $"$ & $"$. & " \\
\hline 54 & PSEUDOCHEIRUS & 앙 & SEPT. 10 & $"$ & " & N.E. SLOPES & c. $6500 \mathrm{FT}_{7}$. \\
\hline 16455 & $11193151=$ & P.YG. & $"$ & $"$ & " & " & " \\
\hline 56 & 1191103 & q & $"$ & $"$ & $"$ & " & $\because$ \\
\hline 57 & Rattus EX. & 우 & $"$ & $"$ & $"$ & HAGAV̈E CR. & c. $6000 \mathrm{FT}$. \\
\hline 58 & " 191587 & $\sigma$ & $"$ & $"$ & $"$ & $"$ & . \\
\hline 59 & $\Rightarrow \quad 192637$ & 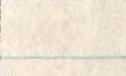 & $"$ & $"$ & $"$ & $"$ & " \\
\hline 16460 & Otomops & ઈ & " & $"$ & $"$ & $"$ & c. $6500 \mathrm{FT}$. \\
\hline 61 & PoGONOMYS (wH.T) & $q$ & .. & $"$ & " & 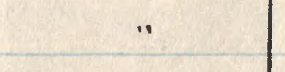 & " \\
\hline 62 & Rattus $N^{19 / 813}$ & $A$ & " & $"$ & " & " & " \\
\hline 63 & SYYONYCTERIS & $\sigma$ & " & $"$ & . & $"$ & " \\
\hline 64 & .192782 & $q$ & " & " & $"$ & $"$ & " \\
\hline 16465 & 192483 & 8 & " & " & $\because$ & " & . \\
\hline 66 & MELOMYS $192065($ W. B. $)$ & $\hat{o}$ & SEPT. $\|$ & 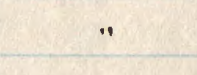 & $"$ & $"$ & " \\
\hline 67 & " 192066. & $\delta$ & " & $"$ & " & $"$ & " \\
\hline 68 & Melomys (SPRDY) & $\hat{0}$ & " & $"$ & " & "H.CR. & " \\
\hline 69 & Rattus Ex. & $\hat{\sigma}$ & $"$ & $"$ & " & "H.CR & “ \\
\hline 16470 & PoGONOMYS & im. $\hat{z} ?$ & $"$ & " & $"$ & " & \\
\hline
\end{tabular}

Hagave Creek comes DOWN THE N.E. SLOPES OF Mt. Michael HUST E. OF CLEON LAUGHLINS FaIth MISSION. ABOUT 3 ROAD MILES $W$. OF OUR CAMP IN BEECH FOREST NEAR THICK'S SAWMILL: 


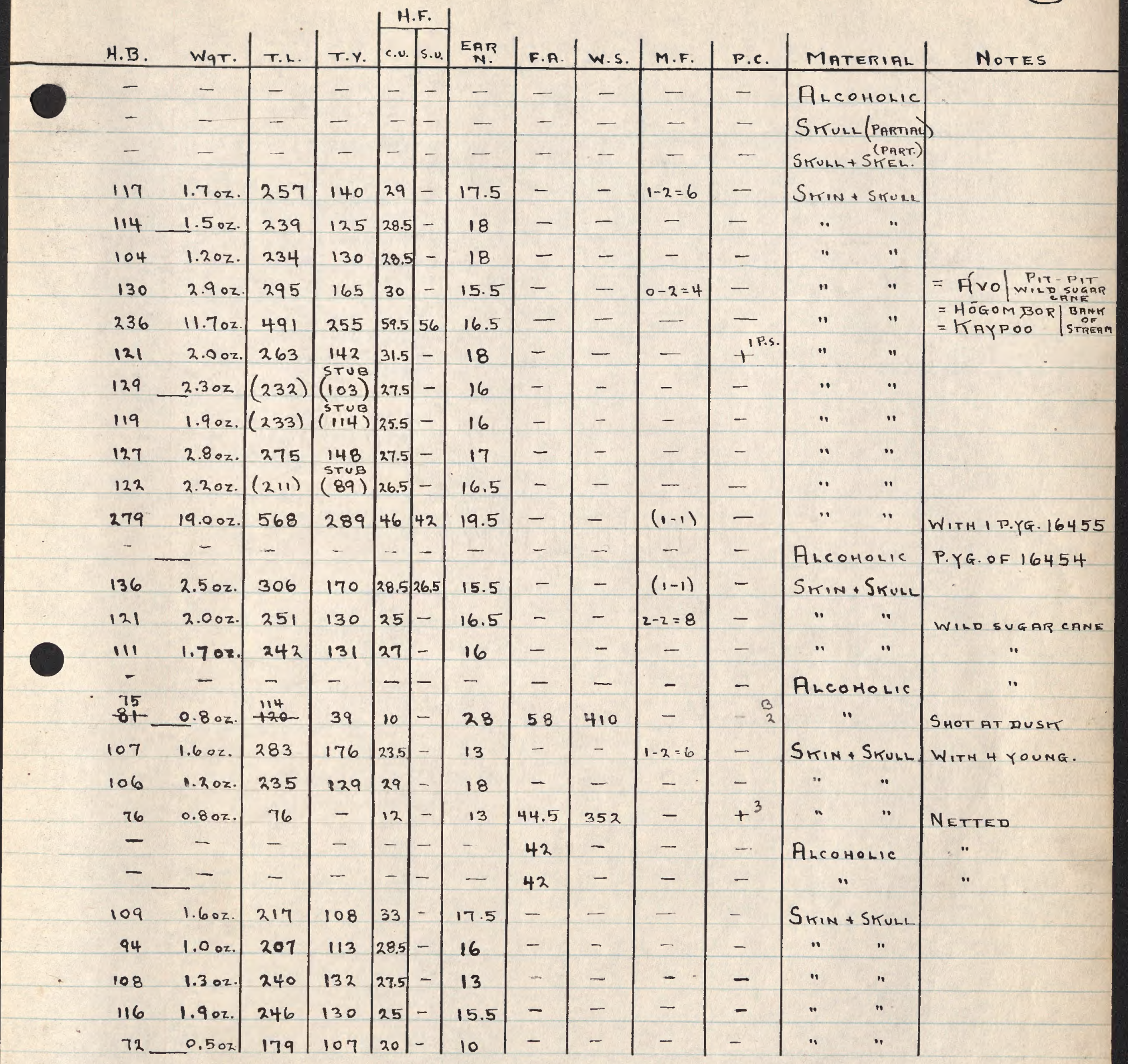



(50)

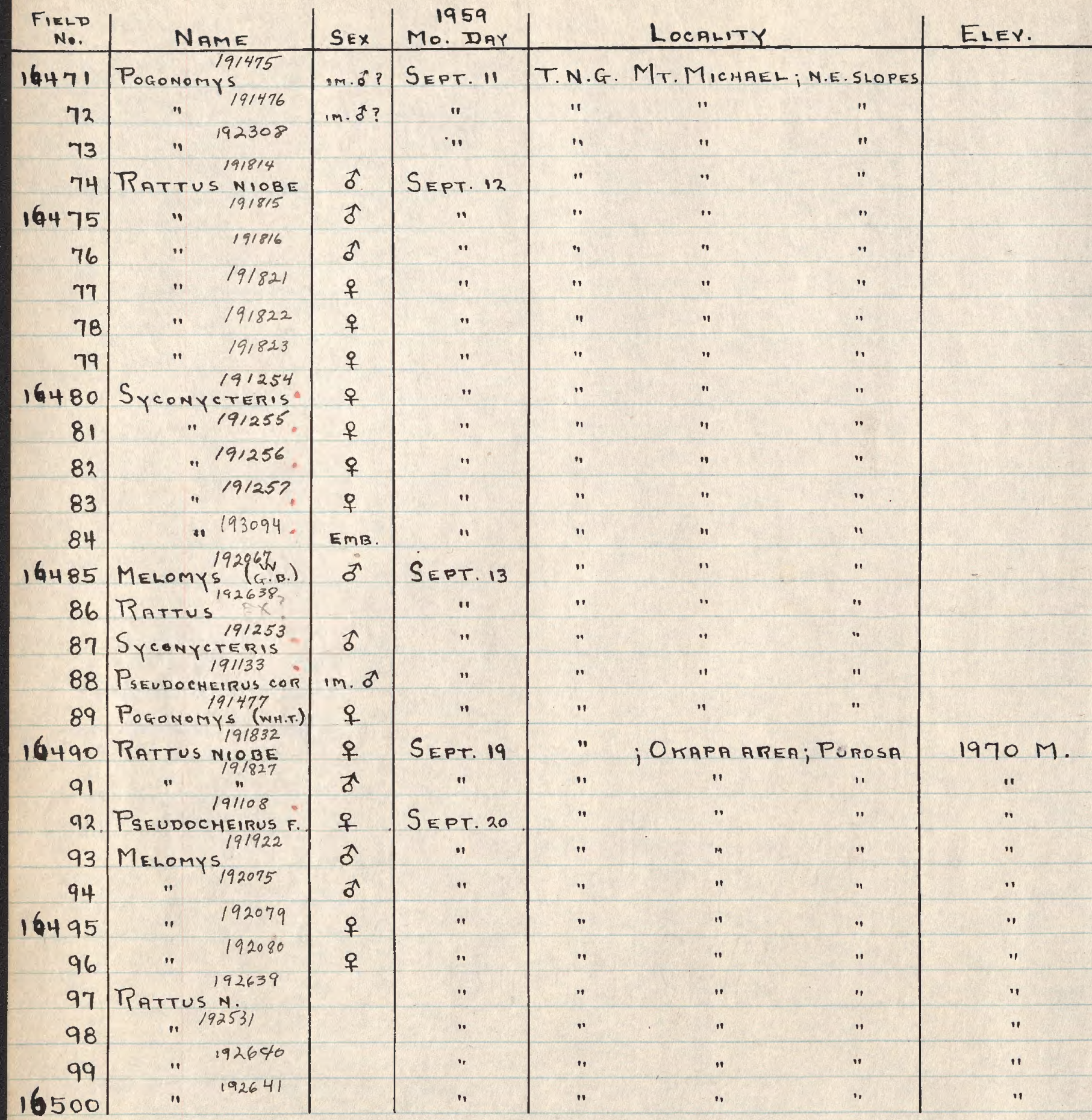




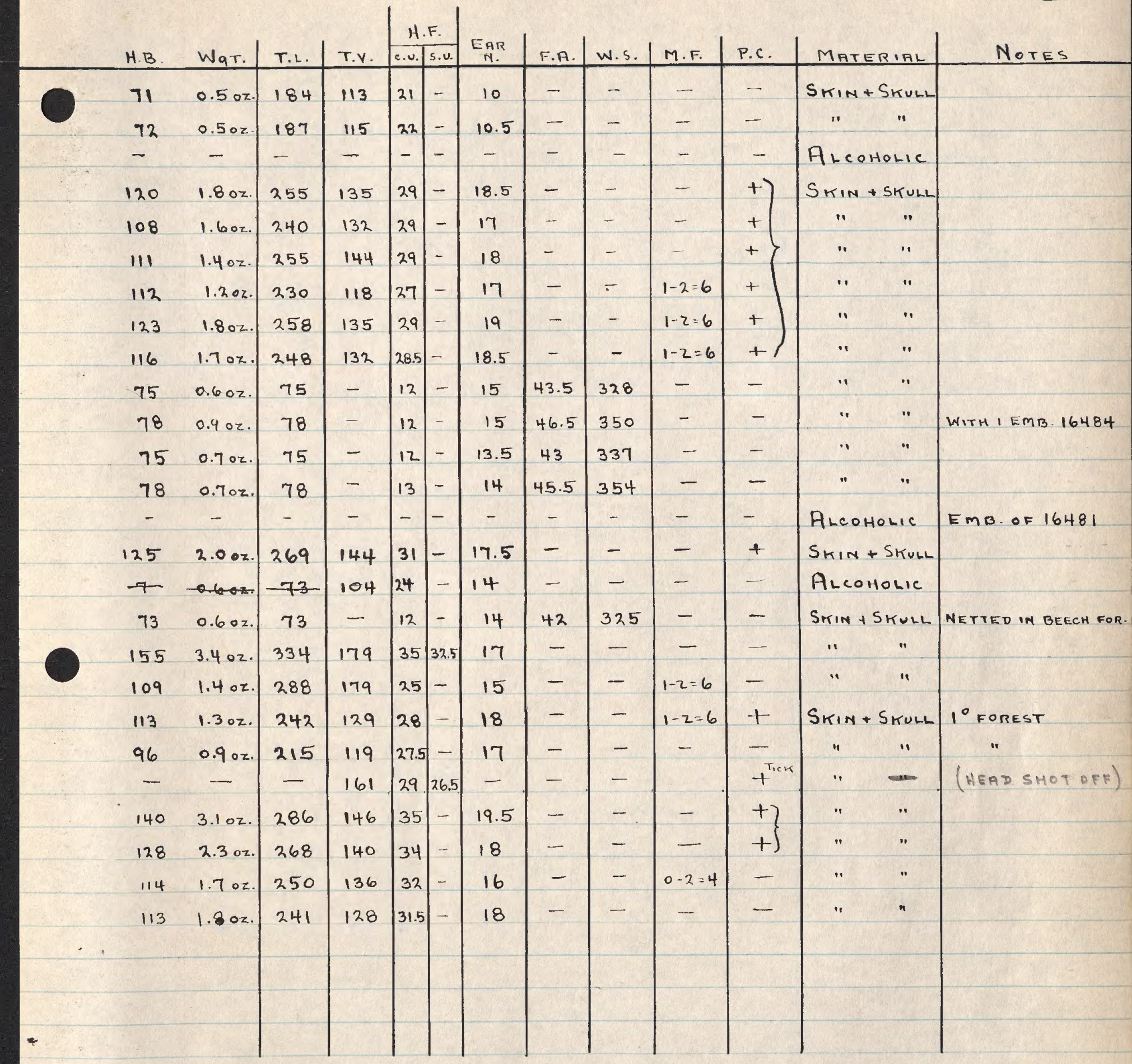



-

-

- 
(51)

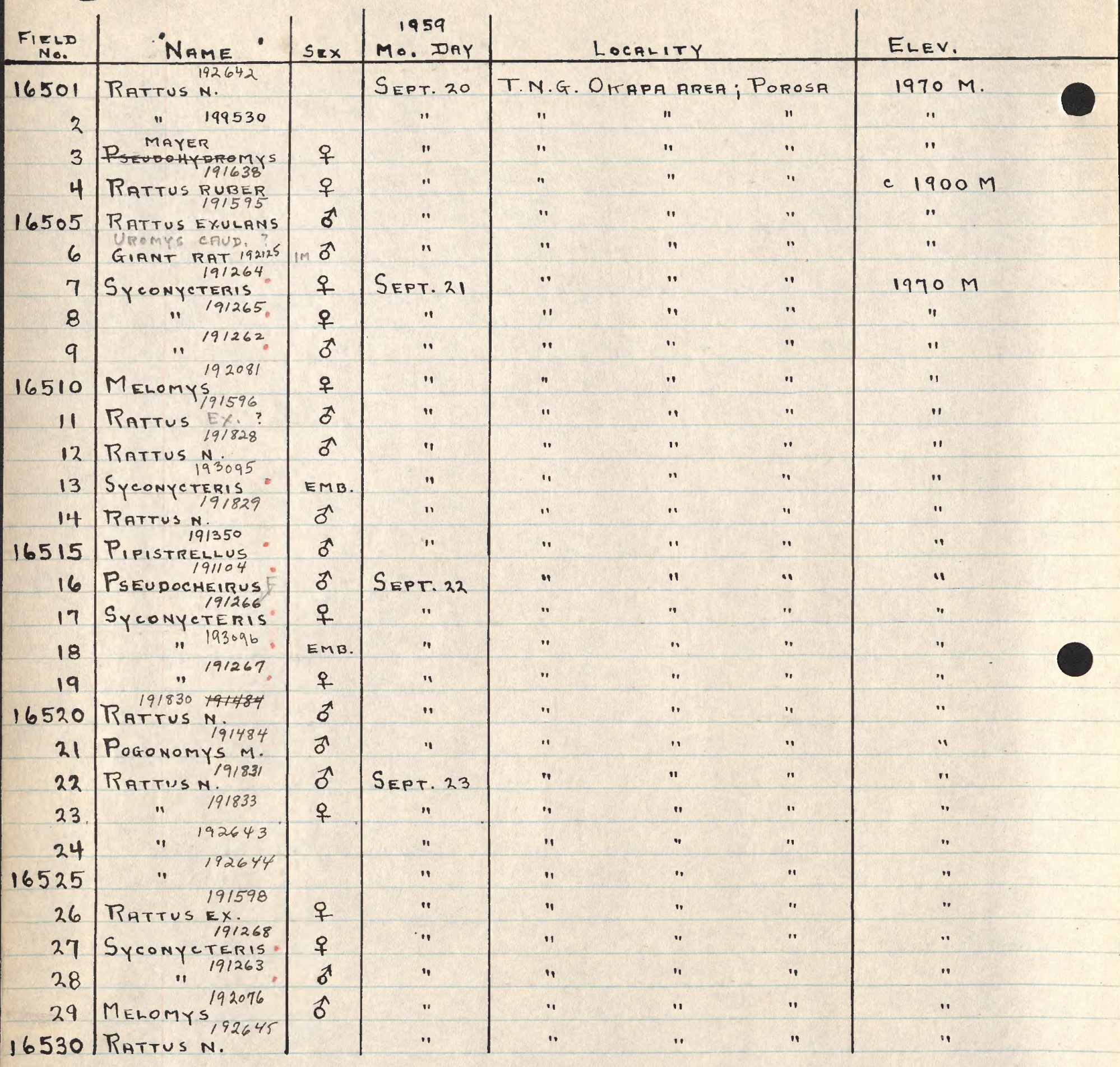

153 
(51)

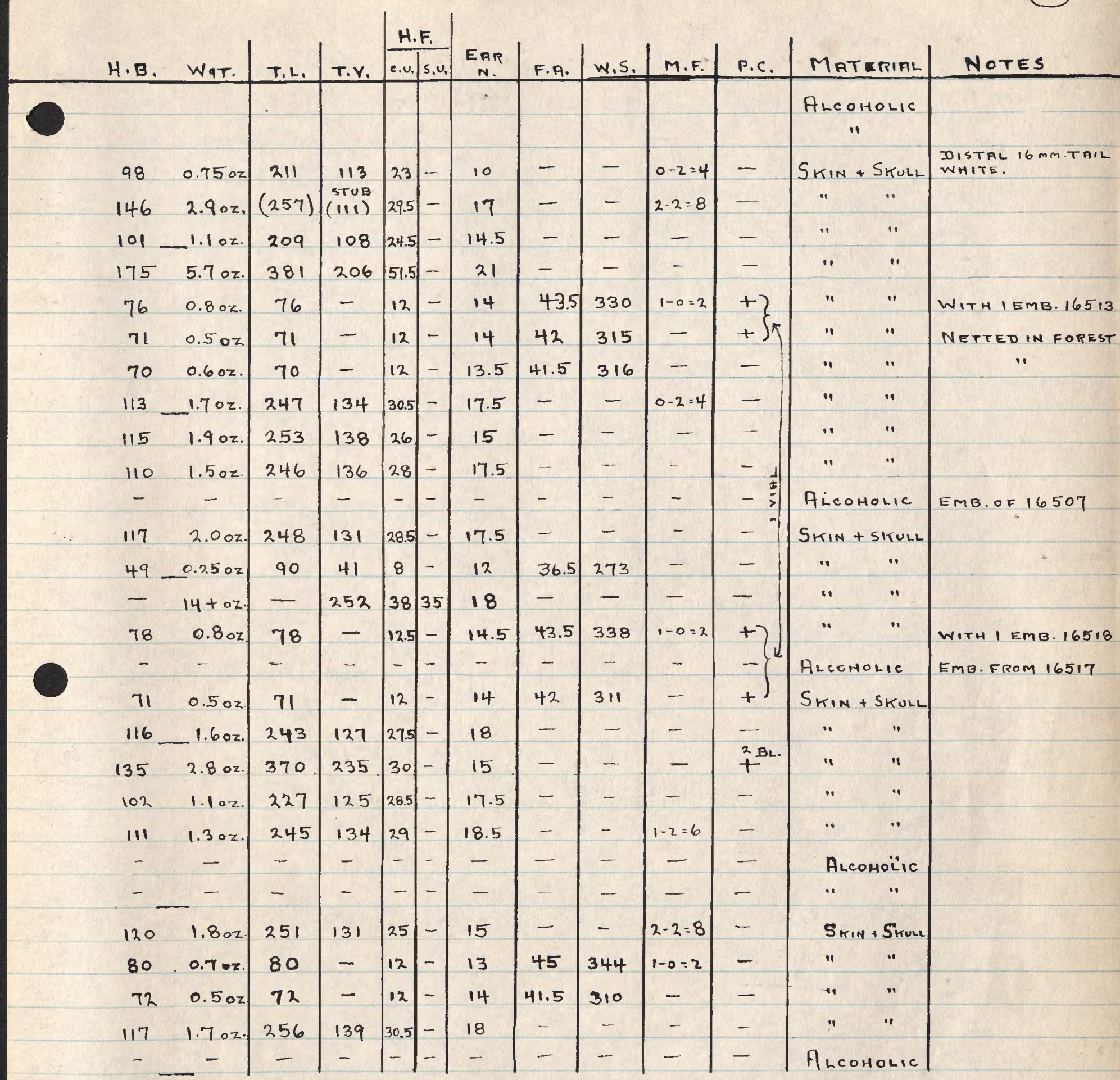






\begin{tabular}{|c|c|c|c|c|c|c|c|c|c|c|c|c|}
\hline \multirow[b]{2}{*}{$\mathrm{H} \cdot \mathrm{B}$. } & \multirow[b]{2}{*}{ Wat } & \multirow[b]{2}{*}{ T.L. } & \multirow[b]{2}{*}{ T.V. } & \multicolumn{2}{|c|}{ H.F. } & \multirow{2}{*}{$\begin{array}{c}E_{A R} \\
N\end{array}$} & \multirow{2}{*}{ F.A. } & \multirow[b]{2}{*}{ w.s. } & \multirow[b]{2}{*}{ M.F. } & \multirow[b]{2}{*}{ P.C. } & \multirow[b]{2}{*}{ MATERIAL } & \multirow[b]{2}{*}{ NOTES } \\
\hline & & & & c.u. & 5.u. & & & & & & & \\
\hline - & - & - & - & - & - & - & - & - & - & - & AlCOHOLIC & \\
\hline - & - & - & - & - & - & - & - & - & - & - & $"$ & \\
\hline - & - & - & - & - & - & - & - & - & - & - & ". & \\
\hline - & - & - & - & - & - & - & - & - & - & - & " & \\
\hline - & - & - & - & - & - & - & - & - & - & - & " & \\
\hline 147 & 3.3 oz. & 360 & 213 & 32 & - & 17 & - & - & - & - & SKIN+SKULL & \\
\hline 129 & $2.70 z$. & 309 & 180 & 30 & - & 17.5 & - & - & $0-2=4$ & - & $"$ & \\
\hline 155 & $4.00 z$. & $(238)$ & $(83)$ & 36.5 & - & 19.5 & - & - & - & - & " & \\
\hline 129 & $2.3 \mathrm{oz}$. & 263 & 134 & 32.5 & - & 18 & - & - & $0-2=4$ & - & $"$ & \\
\hline 233 & $11.70 z$ & 547 & 314 & 52.5 & 48 & 27 & - & - & $(1-1)$ & - & $" \quad \quad{ }^{i}$ & N.F. \\
\hline+32 & $\begin{array}{lll}5 & L B \\
3 & \circ & Z\end{array}$ & 818 & 386 & 73 & 65 & 25 & - & - & $(2-2)$ & - & $"$ & \\
\hline - & - & - & - & - & - & - & - & - & - & - & Alcohome & \\
\hline 291 & $\begin{array}{l}\text { ZLB. } \\
7.3 \mathrm{oz} .\end{array}$ & 618 & 327 & 47.5 & 43 & 19.5 & - & - & - & - & SKIM + SKULL & \\
\hline 273 & $17.6 \mathrm{oz}$ & 545 & 272 & 42.5 & 39 & 19.5 & - & - & $(1-1)$ & - & $"$ & \\
\hline 322 & $20 z$ & 625 & 303 & 57 & 52 & 24 & - & - & & - & $"$ & $1 P \cdot Y G, \quad 16546$ \\
\hline- & - & 一 & - & - & - & - & - & - & - & - & AleOHOLIC & (P.YG. OF 16545$)$ \\
\hline 112 & $1.4 \mathrm{oz}$ & 295 & 183 & 26.5 & - & 17 & - & - & $1-2=6$ & $t^{I F .}$ & SkIN + SkuL & \\
\hline 328 & 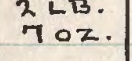 & 711 & 383 & 77 & 71 & 24 & - & - & - & - & $\ddot{*}$ & \\
\hline - & - & - & - & -1 & - & 一 & - & - & - & - & AlComoLIC & \\
\hline - & - & - & - & - & - & - & - & - & - & - & $"$ & \\
\hline - & - & - & - & - & - & - & - & - & - & - & $"$ & \\
\hline - & - & - & - & - & - & - & - & - & - & - & $1 "$ & \\
\hline - & - & - & - & - & - & - & - & - & - & - & " & - \\
\hline 150 & $3.6 \mathrm{oz}$ & 324 & 174 & 38 & - & 20 & - & - & - & - & SKIN + SKULL & \\
\hline 147 & 3.007 & 307 & 160 & 36.5 & - & 19 & - & - & $0-2=4$ & - & $"$ & \\
\hline 140 & 3.302. & 301 & 161 & 35 & - & 19 & - & - & $0-2=4$ & - & $"$ & \\
\hline 91 & 0.9 oz. & 191 & 100 & 28.5 & - & 15 & - & - & - & - & $"$ & \\
\hline - & - & - & - & - & - & - & - & - & - & - & Aleoholic & \\
\hline - & - & - & - & - & - & - & - & - & - & - & $"$ & \\
\hline - & - & - & - & - & - & - & - & - & - & - & " & \\
\hline
\end{tabular}



○

○

0 
(53)

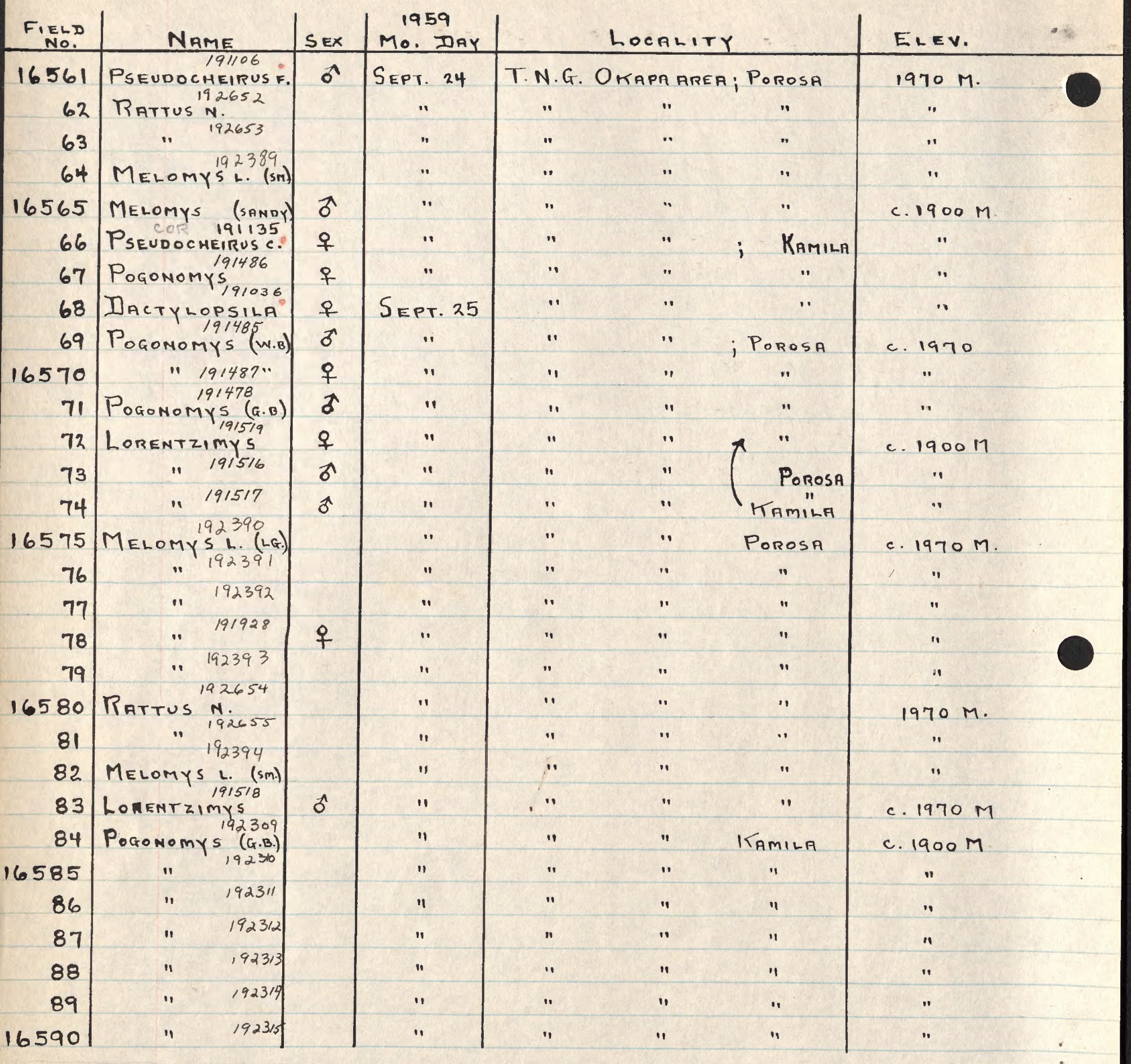

1599 
(53)

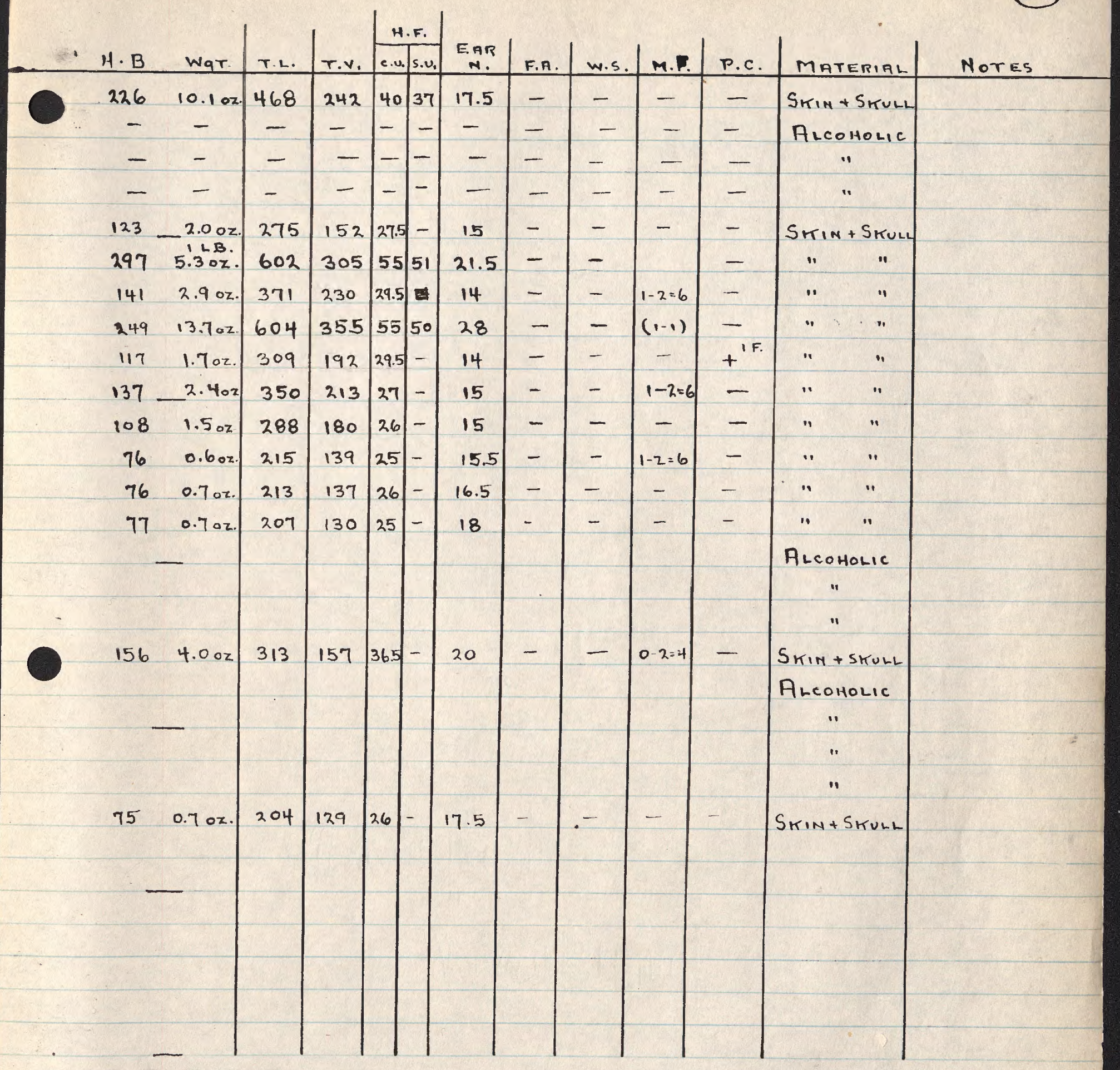




(54)

\begin{tabular}{|c|c|c|c|c|c|c|c|}
\hline $\begin{array}{c}\text { FIELD } \\
\text { NO. }\end{array}$ & \multirow{2}{*}{ INAME } & SEx & $\begin{array}{c}1959 \\
\text { Mo. DAY }\end{array}$ & \multicolumn{3}{|c|}{ LOCALITY } & ELEY. \\
\hline 16591 & & & SEPR. 25 & T.N. & & IMILA & c. $1900 \mathrm{M}$. \\
\hline 92 & " $\quad 192316$ & & $"$ & $"$ & $"$ & $"$ & $"$ \\
\hline 93 & " 192316 & & $"$ & $"$ & $"$ & $"$ & $"$ \\
\hline 94 & MELOMYS (SHAOY) & $\hat{\sigma}$ & " & $"$ & $"$ & $"$ & $\because$ \\
\hline 16595 & RATTUS RUBER & & " & $"$ & $"$ & $"$ & $"$ \\
\hline 96 & 11 192657 & & $"$ & $"$ & $"$ & $"$ & $"$ \\
\hline 197 & $11 \quad 192658$ & & " & $"$ & $"$ & " & $"$ \\
\hline 98 & " 192659 & & $"$ & $"$ & $"$ & $"$ & $"$ \\
\hline 99 & " 192660 & & $"$ & $"$ & $"$ & $"$ & $"$ \\
\hline 16600 & 11 192661 & & $"$ & $"$ & $"$ & $"$ & $"$ \\
\hline 1 & $\begin{array}{r}192662 \\
\text { RATTUS EXULANS } \\
191073\end{array}$ & & $"$ & " & $"$ & $"$ & $"$ \\
\hline 2 & Petaurus & $\delta$ & $"$ & $"$ & $"$ & $"$ & $"$ \\
\hline 3 & $11 \quad 191074$ & $\widetilde{\sigma}$ & " & $"$ & $"$ & $"$ & $"$ \\
\hline 4 & 11 191076 & $q$ & $"$ & $"$ & $\because$ & $" 1$ & $" 1$ \\
\hline 16605 & PoGONOMYS & & $"$ & " & $" 1$ & $"$ & $"$ \\
\hline 6 & " 192283 & & $"$ & $"$ & $"$ & $"$ & $"$ \\
\hline 7 & " $\quad 192284$ & & $"$ & $"$ & $"$ & $"$ & $"$ \\
\hline 8 & $\begin{array}{ll}11 & 192285 \\
192286\end{array}$ & & $"$ & $"$ & $" 1$ & $"$ & " \\
\hline 9 & " $\quad \begin{array}{l}192286 \\
192297\end{array}$ & & $"$ & $"$ & $"$ & " & $"$ \\
\hline 16610 & $\begin{array}{ll}11 & 192297 \\
19721\end{array}$ & & " & $"$ & 11 & $"$ & $" 1$ \\
\hline 11 & $\begin{array}{c}192721 \\
\text { EUDROMIC19 }\end{array}$ & $q$ & $"$ & $"$ & $"$ & " & $"$ \\
\hline 12 & "1 192722 & ๕ठ & in & $"$ & $" 1$ & $"$ & $"$ \\
\hline 13 & 11 192723 & $\delta$ & $"$ & $"$ & $"$ & $"$ & $"$ \\
\hline 14 & " 192924 & 车。 & $"$ & $"$ & $"$ & $"$ & $"$ \\
\hline 16615 & $\begin{array}{r}192725 \\
191405\end{array}$ & $\pi$ & $"$ & $"$ & $"$ & $"$ & $"$ \\
\hline 16 & LEPTOMYS $\frac{191405}{191406}$ & $\hat{\sigma}$ & $"$ & " & $"$ & " & " \\
\hline 17 & $\begin{array}{rr}11 \quad 191406 \\
191407\end{array}$ & $\widehat{0}$ & $"$ & ". & $"$ & $"$ & $"$ \\
\hline 18 & 1192286 & 운 & $\therefore$ & $"$ & $"$ & $" 1$ & 11 \\
\hline 19 & PoG०NOMYS $\left(v \cdot r_{192}(8)\right.$ & & $"$ & $"$ & $"$ & $" 1$ & $"$ \\
\hline 16620 & " & & $" 1$ & $"$ & $"$ & $\because$ & $"$ \\
\hline
\end{tabular}




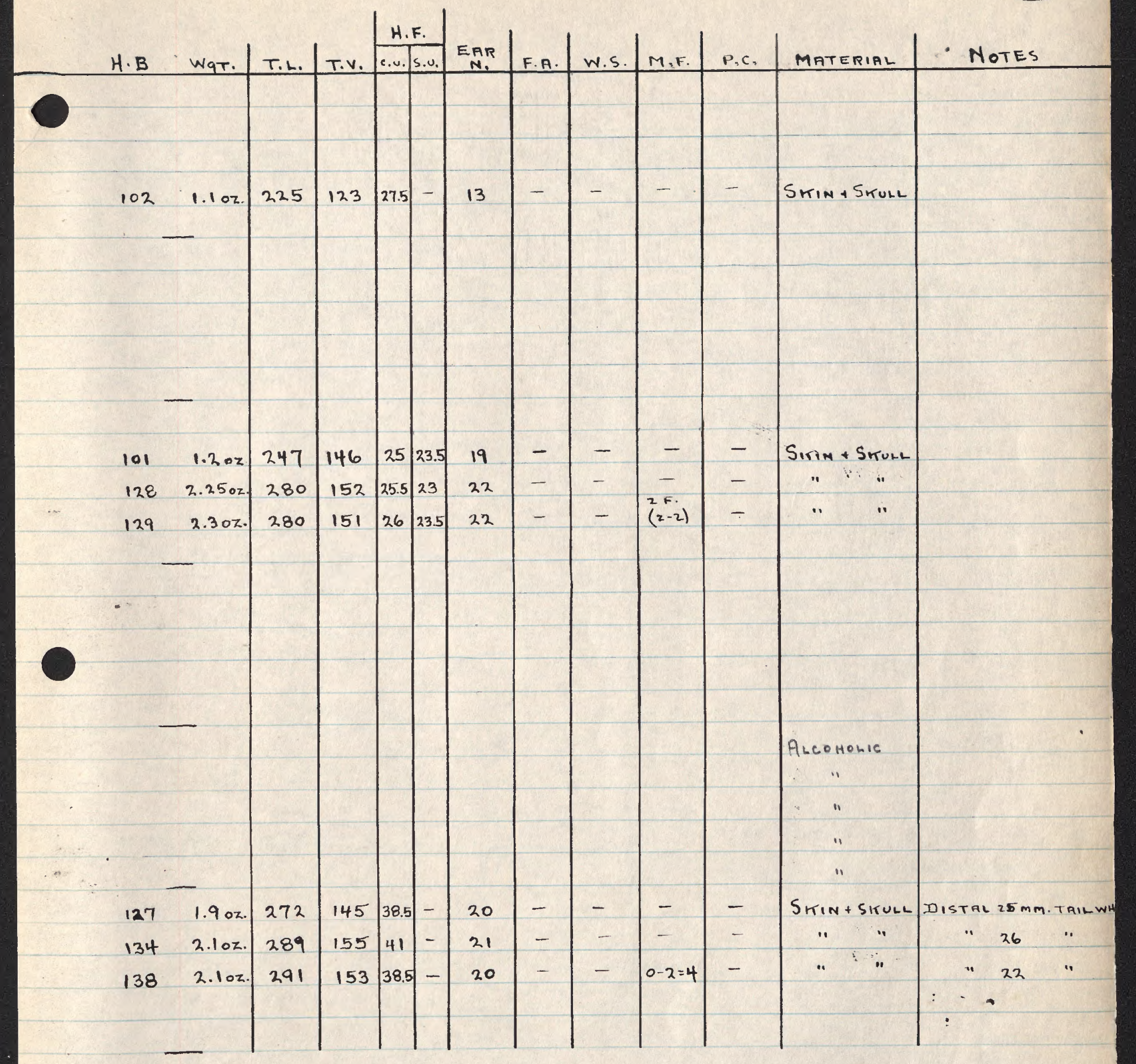





\begin{tabular}{|c|c|c|c|c|c|c|c|c|}
\hline $\begin{array}{c}\text { FIELD } \\
\text { NO. }\end{array}$ & NAME & SEX & Mo. DAY DA & \multicolumn{3}{|c|}{ LaCALITY } & \multicolumn{2}{|l|}{ ELEV: } \\
\hline 16621 & Pogonomys $(4, \pi)$ & & Sepr. 25 & T.N.G & $P A$ & ; MAMILA & c. $1900 \mathrm{~m}$. & \\
\hline 22 & " 192289 & & $"$ & . & $"$ & $\because$ & * & \\
\hline 23 & " 192248 & & $"$ & $\because$ & $\because$ & 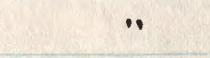 & 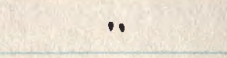 & \\
\hline 24 & $\because \quad 192299$ & & $"$ & $"$ & $\because$ & ". & ". & \\
\hline 16625 & PoGONOMYS (GR) & & $"$ & $"$ & $"$ & $\because$ & $"$ & \\
\hline 26 & . $\quad 192319$ & & $"$ & $"$ & $"$ & $"$ & $"$ & \\
\hline 27 & $\because \quad 192320$ & & $"$ & " & $\because$ & " & $"$ & 4 \\
\hline 28 & ․ 192321 & & $"$ & " & $"$ & $"$ & $\because$ & \\
\hline 29 & " 192322 & & $"$ & " & ". & “ & " & \\
\hline 16630 & $\begin{array}{cc}192323 \\
-\quad 199521\end{array}$ & $q\}$ & $"$ & $"$ & $"$ & 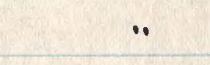 & " & \\
\hline 31 & $\begin{array}{cc}* & 199521 \\
\cdots & 192290\end{array}$ & im. & $"$ & " & ". & " & 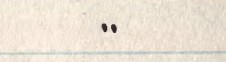 & \\
\hline 32 & Pogonomys $\begin{array}{r}19290 \\
\text { (Y.T.) }\end{array}$ & & $"$ & $"$ & $"$ & $"$ & $\because$ & \\
\hline 33 & " 192291 & & " & $"$ & $"$ & " & " & \\
\hline 34 & $\because \quad 192292$ & & $"$ & $\because$ & $"$ & $"$ & $"$ & \\
\hline 16635 & $\begin{array}{l}190962 . \\
\text { Peroryctes }\end{array}$ & $q$ & SEPT. 26 & " & $\because$ & Porosh & $1970 \mathrm{M}$. & \\
\hline 36 & Eudromicia & $q$ & $"$ & 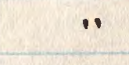 & $"$ & KaMILA & c. $1900 \mathrm{M}$. & \\
\hline 37 & " 191032 & im. $q$ & $"$ & $"$ & " & " & $"$ & \\
\hline 38 & SYCONYCTERIS. & & ". & " & 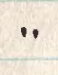 & Porosa & $1970 \mathrm{M}$. & \\
\hline 39 & $\because 192789$ & & $"$ & $"$ & 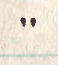 & 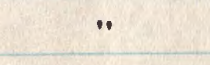 & • & \\
\hline 16640 & MELOMYS L. (sm.) & & $"$ & $"$ & $"$ & " & $"$ & \\
\hline 41 & RatTus 192663 & & " & $"$ & " & " & $"$ & - \\
\hline 42 & " 192664 & & 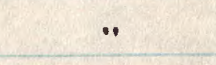 & " & $"$ & $"$ & " & 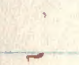 \\
\hline 43 & "1192665 & & $"$ & " & " & " & " & \\
\hline 44 & $\begin{array}{r}192666 \\
1192533\end{array}$ & & " & $"$ & $"$ & 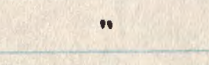 & $"$ & \\
\hline 16645 & " 142533 & & " & $"$ & $"$ & $"$ & $\because$ & . \\
\hline 46 & Rattus ruber & & $"$ & $"$ & $"$ & MAMILA & $"$ & \\
\hline 47 & Pogonomys ( WH. & & 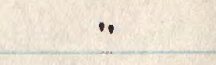 & $"$ & " & $"$ & " & \\
\hline 48 & . $192294 " 10$ & & $"$ & $"$ & " & " & 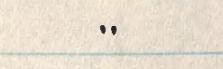 & , \\
\hline 49 & 1192275 " & & $"$ & $"$ & ". & " & " & \\
\hline$|6650|$ & MELOMY'192396 LGG) & & $"$ & $"$ & $"$ & $"$ & 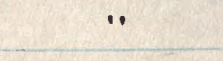 & 1 \\
\hline
\end{tabular}



(56)

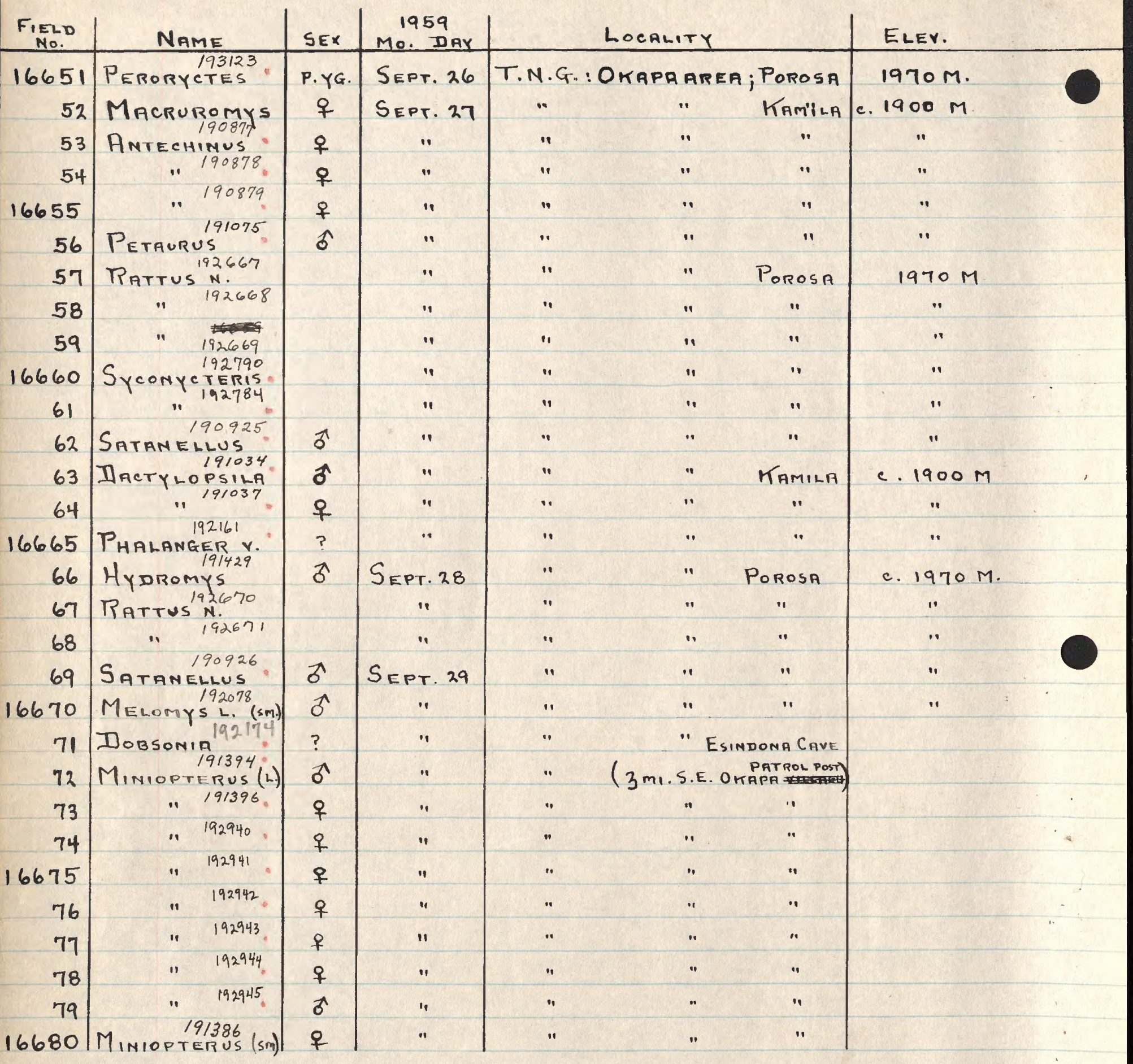

Visit to cave Momoay. Sept. 28 (4:30 P.M.) cave about I mile s.e Illafo villatage

1680 
(56)

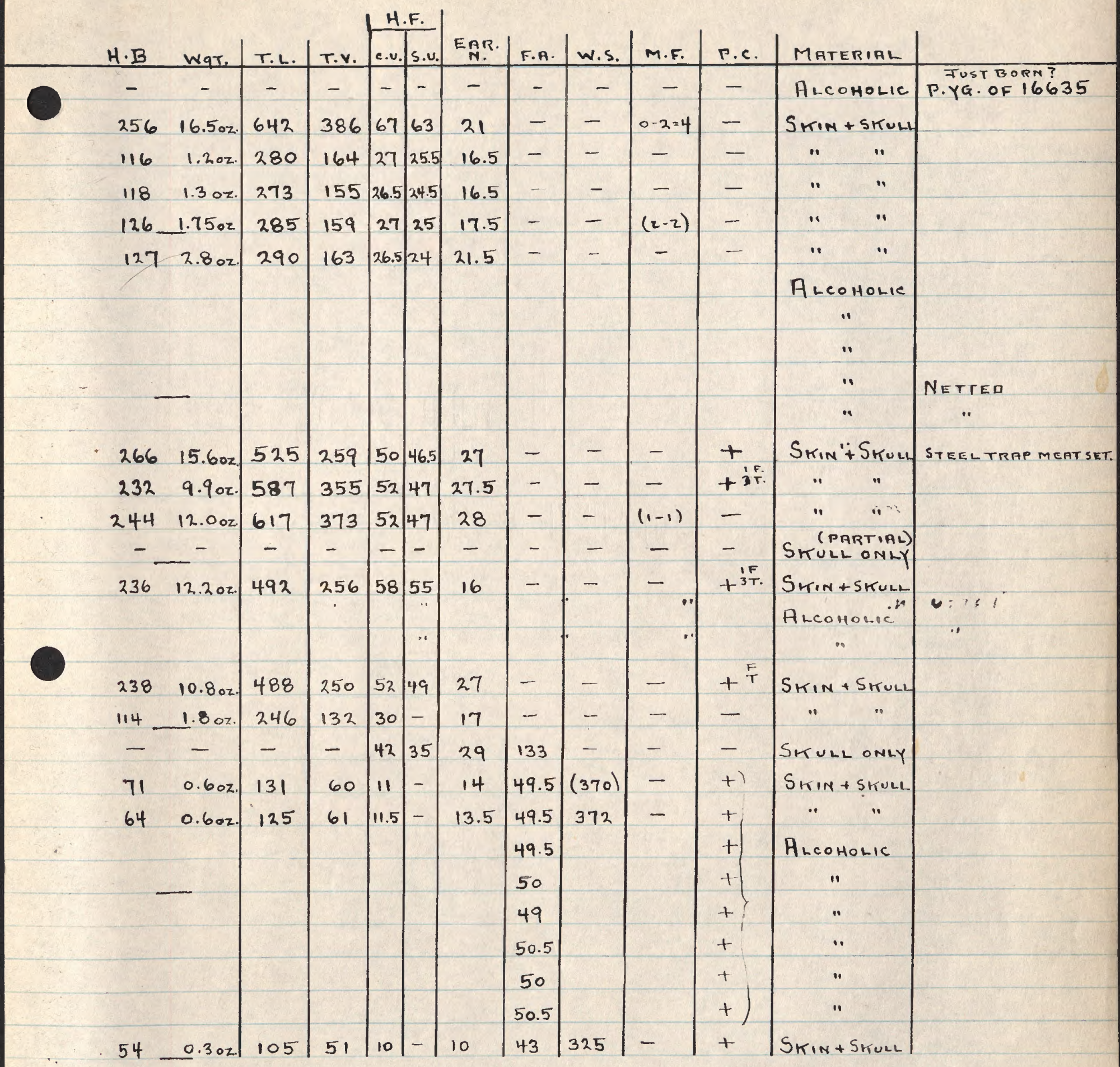




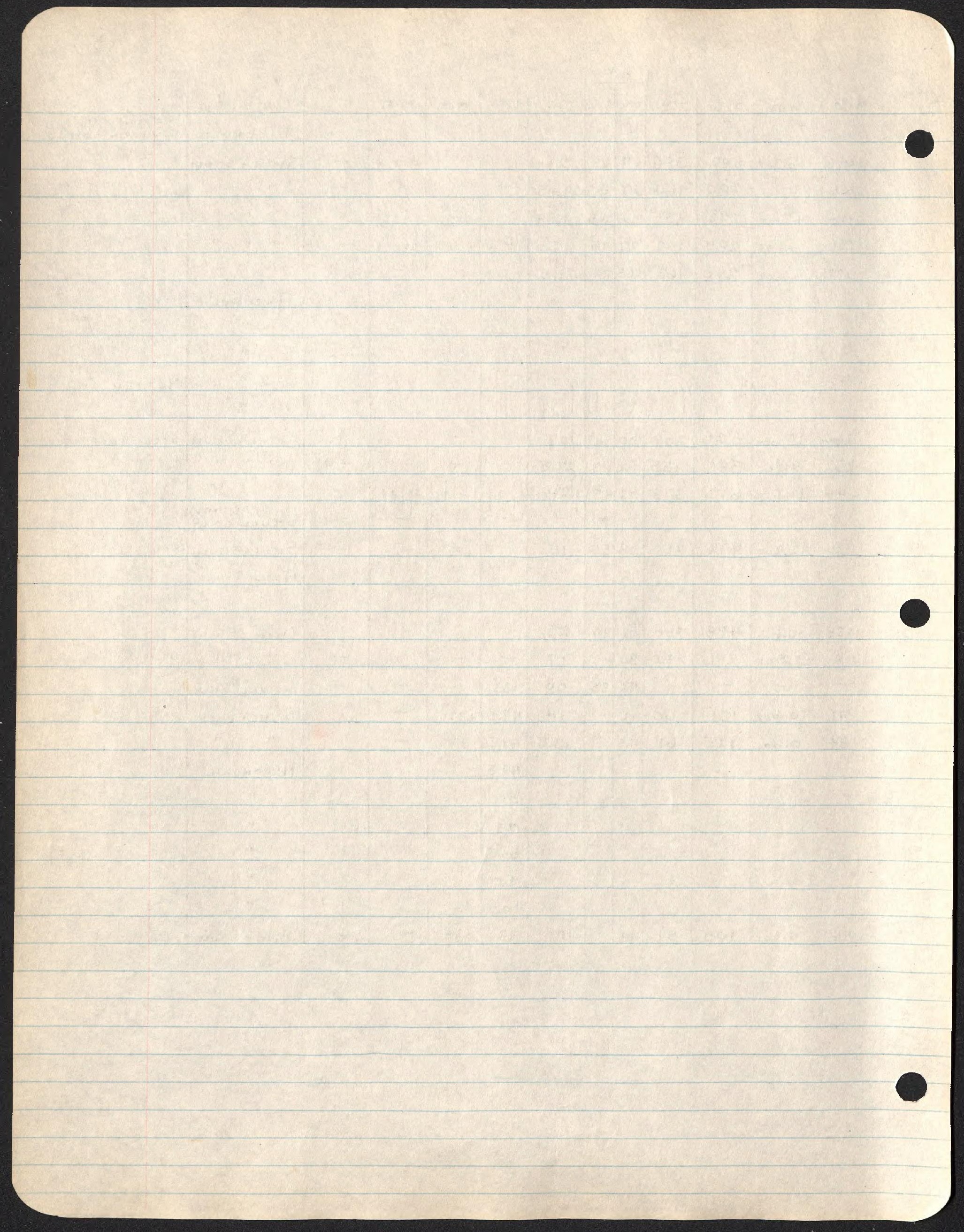



(57)

\begin{tabular}{|c|c|c|c|c|c|c|c|}
\hline $\begin{array}{c}\text { FIELD } \\
\text { NO. }\end{array}$ & NAmE & SEX & mo. 1959 & \multicolumn{3}{|c|}{ LOCALITY } & ELEV. \\
\hline 16681 & $\begin{array}{c}193031 \\
\text { MINIOPTERUS (sm) }\end{array}$ & & SEPT. 29 & T.N.G & & $\begin{array}{l}\text { INDONA } \\
\text { TAYE }\end{array}$ & \\
\hline 82 & " 19001 & $\hat{\sigma}$ & " & $"$ & $"$ & & \\
\hline 83 & " 193015 & $\hat{\sigma}$ & $"$ & $"$ &. & & \\
\hline 84 & $\begin{array}{l}193016 \\
=\quad 103030\end{array}$ & $\widehat{\sigma}$ & $"$ & $"$ & $"$ & $"$ & \\
\hline 16685 & . 193032 & $q$ & $"$ & " & " & " & \\
\hline 86 & " 193033 & 우 & $"$ & $"$ & $"$ & " & \\
\hline 87 & " 193034 & q & $"$ & $"$ & $"$ & " & \\
\hline 88 & . 193035 & q & 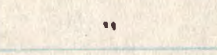 & $"$ & $"$ & ." & \\
\hline 89 & " 193036 & $q$ & $"$ & $"$ & $"$ & $"$ & \\
\hline 16690 & " 193037 & $q$ & $"$ & $"$ & $"$ & " & \\
\hline 91 & " 193038 & 우 & $"$ & $"$ & $"$ & " & \\
\hline 92 & " 193017 & $\sigma$ & $"$ & $"$ & $"$ & $"$ & \\
\hline 93 & . $1930 / 8$ & $\hat{\sigma}$ & $"$ & $"$ & $"$ & " & \\
\hline 94 & " 193039 & 우 & $"$ & 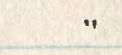 & $"$ & $"$ & \\
\hline 16695 & " 193040 & q & $"$ & $"$ & $"$ & " & \\
\hline 96 & " 193041 & 우 & $"$ & $"$ & $"$ & " & \\
\hline 97 & " $\quad 193042$ & $q$ & $"$ & $"$ & $"$ & $"$ & \\
\hline 98 & $\begin{array}{r}193043 \\
193044\end{array}$ & ㅇ & $"$ & " & $"$ & " & \\
\hline 99 & $\begin{array}{l}193044 \\
193045\end{array}$ & ㅇ & $"$ & $"$ & $"$ & $"$ & \\
\hline 16700 & 1. 193045 & 우 & $"$ & $"$ & $"$ & $"$ & \\
\hline 1 & "193019 & ठ & $"$ & " & $"$ & $"$ & \\
\hline 2 & $\begin{array}{r}193046 \\
=193047\end{array}$ & 우 & $"$ & 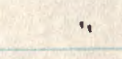 & " & $"$ & \\
\hline 3 & = 193047 & 우 & $"$ & " & $"$ & $\bullet$ & \\
\hline 4 & $\begin{array}{r}193020 \\
193048\end{array}$ & ठ & " & $"$ & " & " & \\
\hline 16705 & $\begin{array}{r}193048 \\
\quad 193049\end{array}$ & $q$ & $"$ & $"$ & . & $"$ & \\
\hline 6 & $\begin{array}{l}193047 \\
193050\end{array}$ & q & $"$ & " & " & " & \\
\hline 7 & $" 190030$ & $q$ & $"$ & $"$ & " & " & \\
\hline 8 & " $19305 !$ & 오 & $"$ & $"$ & ". & $"$ & \\
\hline 9 & 1. 193021 & $\hat{\sigma}$ & $"$ & " & " & . & \\
\hline 16710 & $n^{193022}$ & $\beta$ & 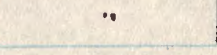 & $"$ & " & " & \\
\hline
\end{tabular}




\begin{tabular}{|c|c|c|c|c|c|c|c|c|c|c|c|c|}
\hline H.B & Wat. & T.L & T.V. & c.u. & s.u. & $\begin{array}{l}\text { EAR } \\
N \text {. }\end{array}$ & F.A. & W.S. & M.F. & P.C. & MATERIAL & Notes \\
\hline & & & & & & & 45 & & & & Alcoholie & \\
\hline & & & & & & & 45 & & & & 11 & \\
\hline & & & & & & & 43.5 & & & & " & \\
\hline & & & & & & & 43.5 & & & & " & \\
\hline & & & & & & & 44 & & & & $\because$ & WITH EMB. \\
\hline & & & & & & & 43.5 & & & & " & $"$ \\
\hline & & & & & & & 45 & & & & " & 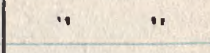 \\
\hline & & & & & & & 43.5 & & & & " & \\
\hline & & & & & & & 43.5 & & & & $\because$ & WITH EMIB. \\
\hline & & & & & & & 44 & & & & $"$ & \\
\hline & & & & & & & 45.5 & & & & $"$ & WITH EMB. \\
\hline & & & & & & & 44 & & & & $"$ & \\
\hline & & & & & & & 43.5 & & & & $\because$ & \\
\hline & & & & & & & 43.5 & & & & $"$ & WITH EMB. \\
\hline & & & & & & & 44 & & & & $" 1$ & $"$ \\
\hline & & & & & & & 43.5 & & & & $\because$ & " \\
\hline & & & & & & & 43.5 & & & & " & $" 1$ \\
\hline & & & & & & & 45 & & & & $"$ & $"$ \\
\hline & & & & & & & 44 & & & & $"$ & " \\
\hline & & & & & & & 45 & & & & $"$ & \\
\hline & & & & & & & 44 & & & & " & \\
\hline & & & & & & & 44 & & & & $"$ & \\
\hline & & & & & & & 44 & & & & $"$ & WITH EMB. \\
\hline & & & & & & & 44 & & & & $"$ & \\
\hline & & & & & & & 45.5 & & & & $"$ & WITH EMB. \\
\hline & & & & & & & 43 & & & & $"$ & $"$ \\
\hline & & & & & & & 43.5 & & & & " & " \\
\hline & & & & & & & 44 & & & & " & $"$ \\
\hline & & & & & & & 44 & & & & $"$ & \\
\hline & & & & & & & 42 & & & & $"$ & \\
\hline
\end{tabular}




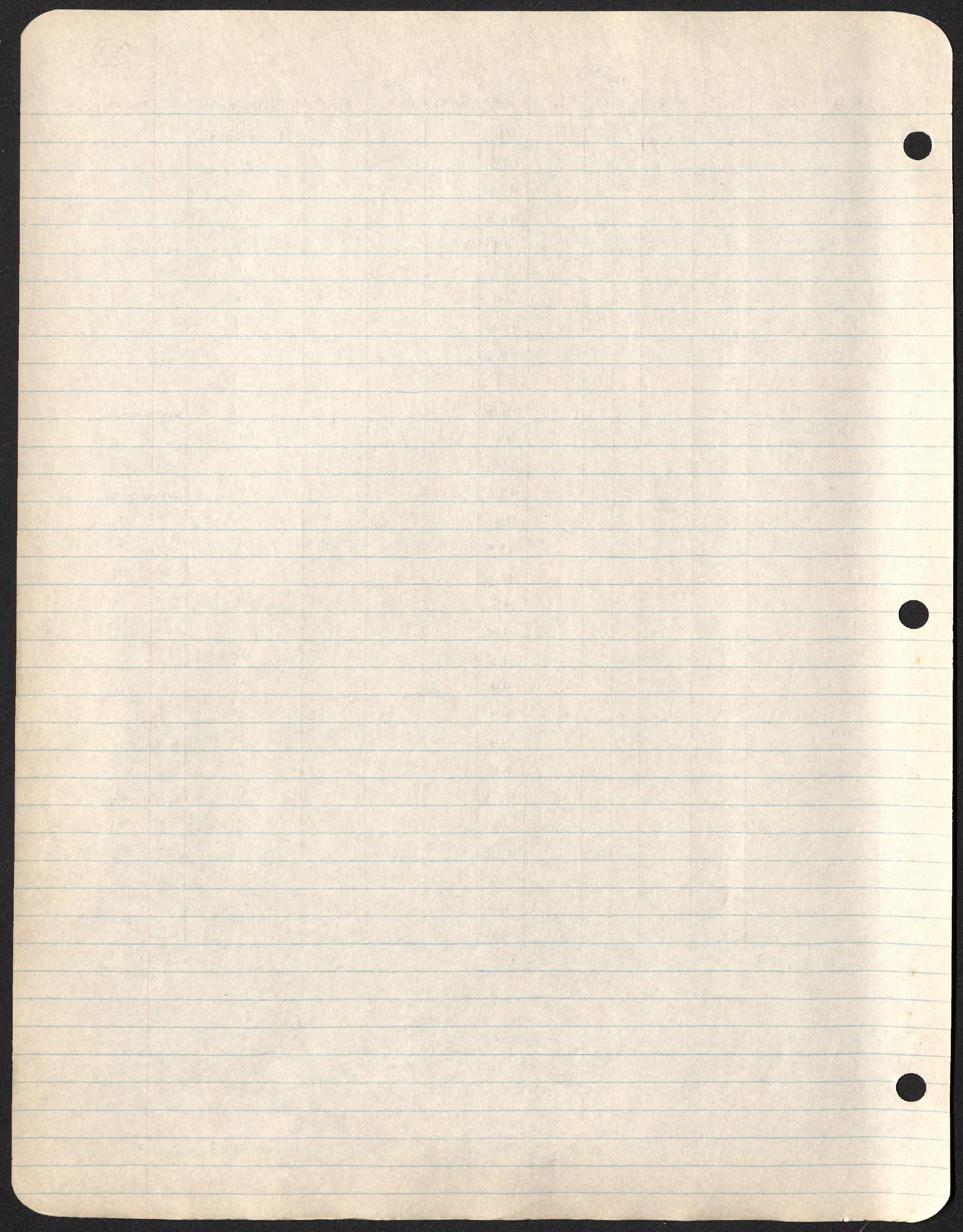





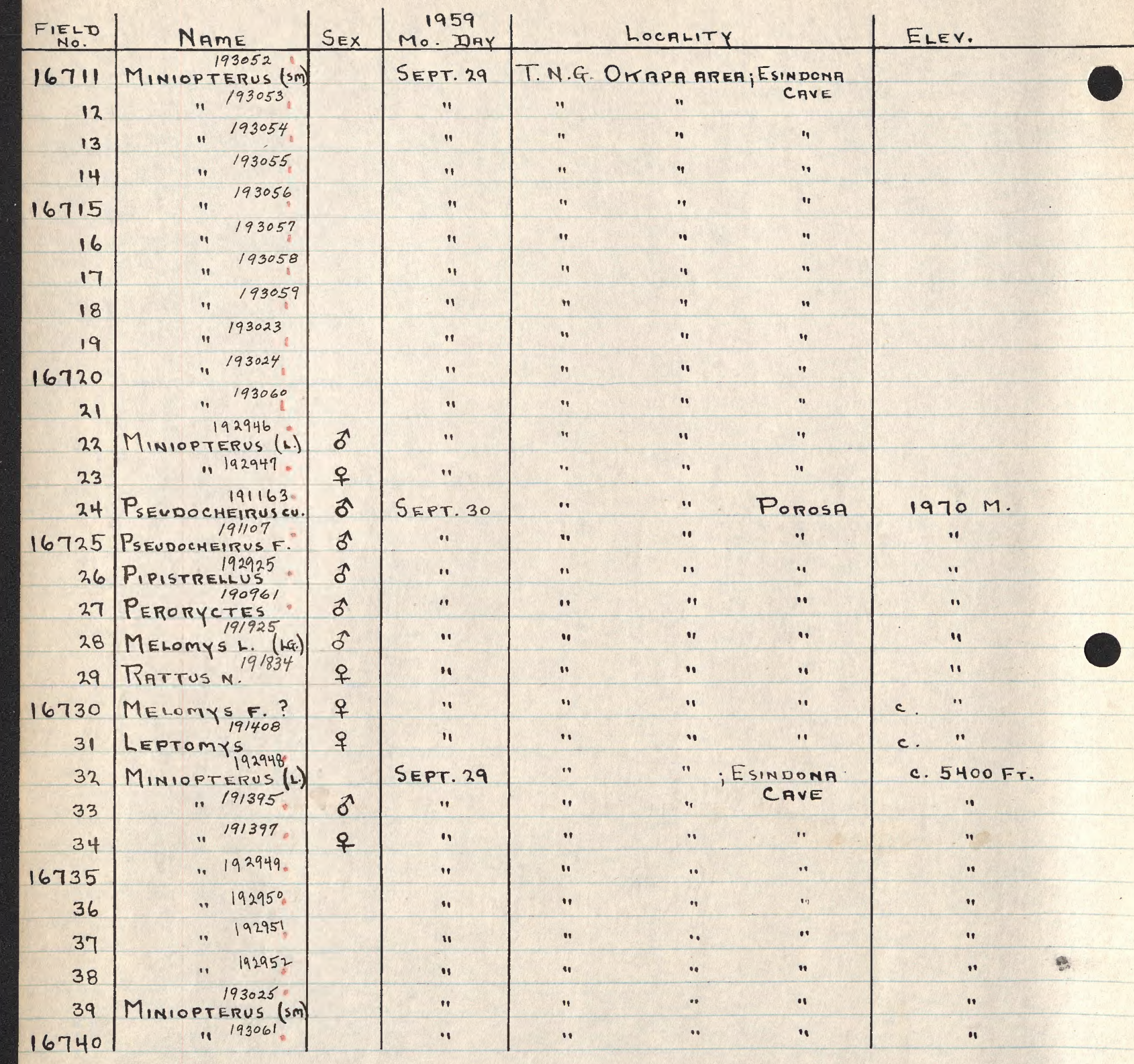

No $16727(190961)$, SEE G.P. HoLLAND (1969), FLEAS OF N.G., P. 48 
(58)

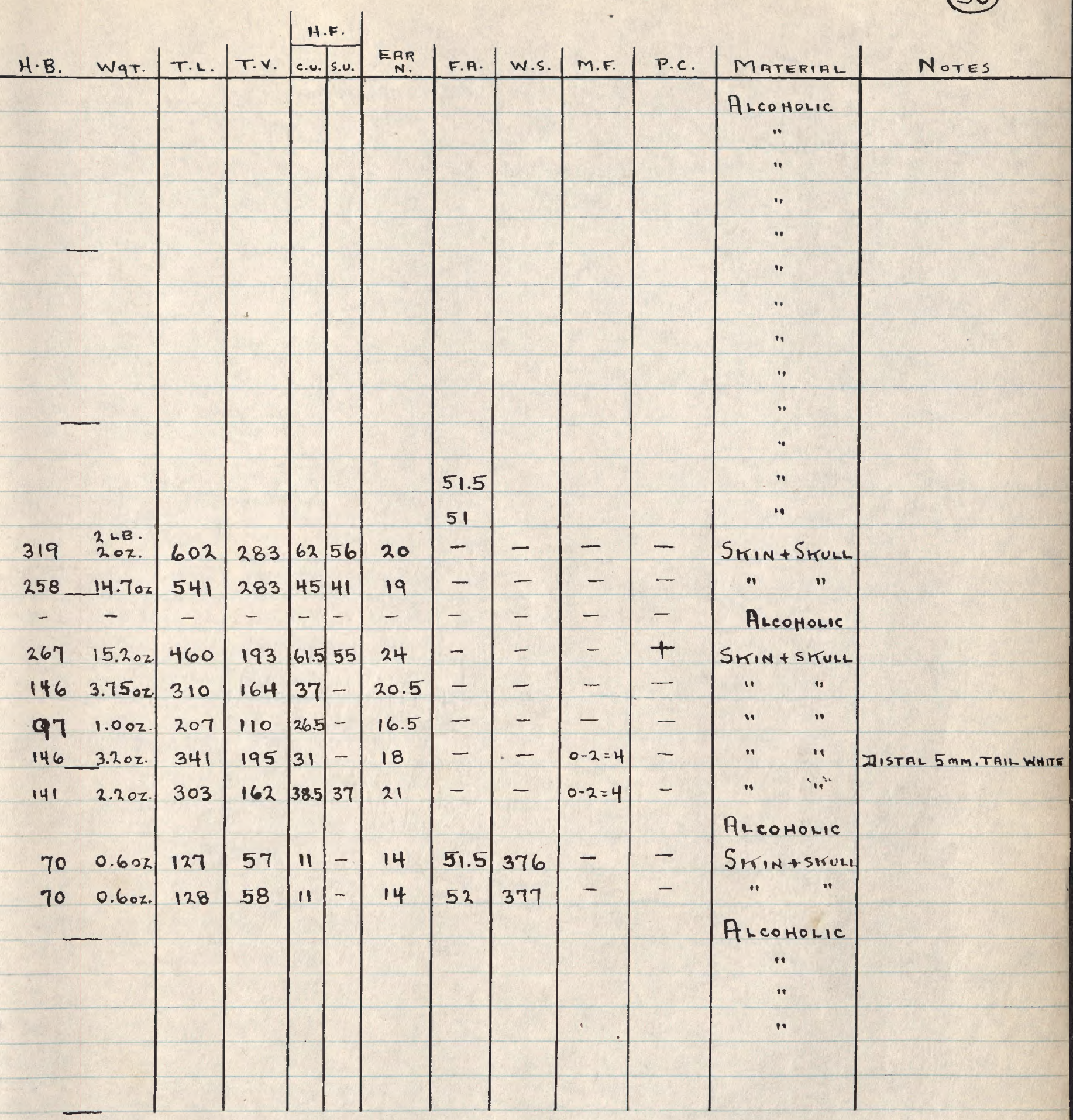


-

- 

(59)

\begin{tabular}{|c|c|c|c|c|c|}
\hline $\begin{array}{ll}F_{1} \in L D \\
\text { NOD }\end{array}$ & Name & SEX & $\begin{array}{l}1959 \\
\text { Mo. DaY }\end{array}$ & LOCALITY & Eley. \\
\hline 16741 & 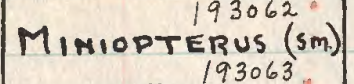 & 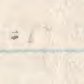 & SEPR. 30 & T.N.G. OKapa aref; Esimpoma care & c. $5400 \mathrm{FT}$. \\
\hline 42 & 193064, & & $"$ & " & \\
\hline 43 & "193026, & & $"$ & " & 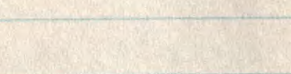 \\
\hline 44 & " 193065 & & $"$ & " & \\
\hline 16745 & 193066 & 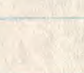 & " & " & \\
\hline $\begin{array}{l}46 \\
47\end{array}$ & 193027 & & " & “ & \\
\hline $\begin{array}{l}47 \\
48\end{array}$ & " 193067 & & " & " & \\
\hline $\begin{array}{l}48 \\
49\end{array}$ & " 193068 & & " & $"$ & \\
\hline 16750 & $n^{19 / 387}$ & q & " & " & \\
\hline 51 & " 193069 & & " & " & \\
\hline 52 & " 193070 & & " & $"$ & \\
\hline 53 & . 19307! & & " & " & \\
\hline 54 & " 193028 & & " & " & \\
\hline 16755 & " $\quad 193072$ & & " & " & \\
\hline 56 & * 193073 & & $\therefore$ & " & \\
\hline 57 & " 193074 & & $"$ & " & \\
\hline 58 & " 193075 & & " & $\cdot$ & \\
\hline 59 & . 193076 & & " & * & \\
\hline 16760 & " $\quad 193077$ & & . & . & \\
\hline 61 & $\Rightarrow \quad 19300^{\prime}$ & & " & " & \\
\hline 62 & " 19/388 & f & " & " & \\
\hline 63 & " 193079 & & " & " & \\
\hline 64 & . ${ }^{193080}$ & & " & " & \\
\hline 16765 & " 19308! & & " & " & \\
\hline 66 & $\left.=\begin{array}{rl}193085 \\
193083\end{array}\right]$ & & " & $"$ & \\
\hline 67 & $\Rightarrow \begin{array}{ll}19300 \\
\quad 193084\end{array} \mid$ & & $"$ & $"$ & \\
\hline $\begin{array}{l}68 \\
69\end{array}$ & 19308.5 & & $"$ & " & \\
\hline $\begin{array}{r}69 \\
16770\end{array}$ & $\Rightarrow 193096$ & & " & ". & \\
\hline
\end{tabular}




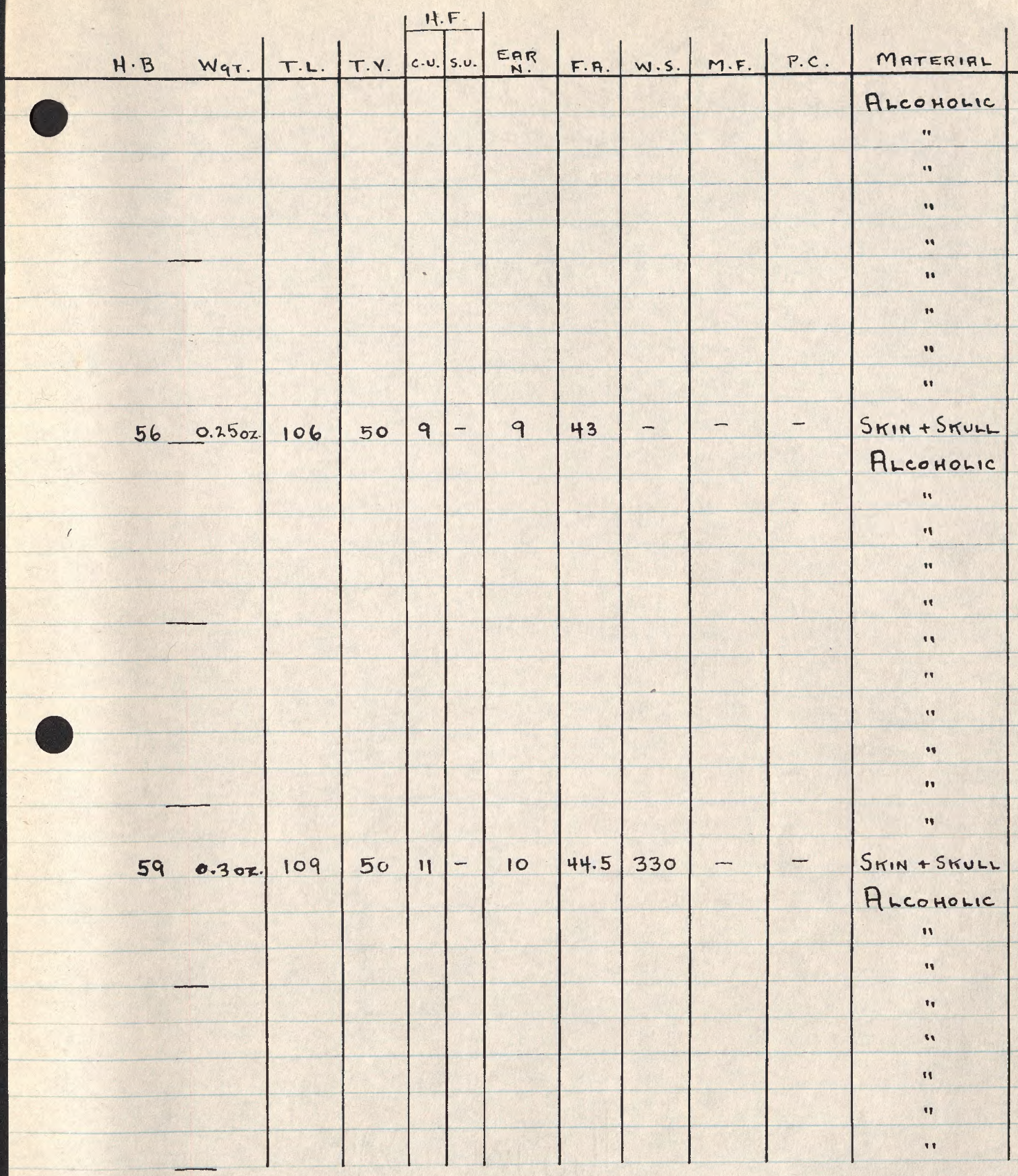




(60)

\begin{tabular}{|c|c|c|c|c|c|c|c|}
\hline $\begin{array}{c}\text { FIELD } \\
\text { No }\end{array}$ & Name & SEX & $\begin{array}{l}1959 \\
\text { Mo. DAY } \\
\end{array}$ & \multicolumn{3}{|c|}{ LOCALITY } & ELEV. \\
\hline 16771 & MiNIOPTERUS 193029$)$ & & SEPT. 29 & \multirow{2}{*}{\multicolumn{3}{|c|}{$\begin{array}{c}\text { T.N.G.: OKAPA AREA; ESINDOMA } \\
" \text { CAVE }\end{array}$}} & c. $5400 \mathrm{Fr}$. \\
\hline 72 & $11^{193030}$ & & $"$ & & & & $"$ \\
\hline 73 & 193087 & & $"$ & \multicolumn{3}{|c|}{$"$} & $"$ \\
\hline 74 & 1193088 & & $"$ & \multicolumn{3}{|c|}{ " } & " \\
\hline 16775 & \multirow{4}{*}{$\begin{array}{c}\text { MELOMYS F. ? } \\
\text { SYCONYCTERIS } \\
\text { RYCORT } 191835 \\
\text { RATTES N. } \\
\text { " } 191836\end{array}$} & 우 & SEPT. 30 & $"$ & $"$ & Porosa & c. $1970 \mathrm{M}$. \\
\hline 76 & & 아 & Oct. 1 & $" 1$ & $"$ & 11 & $1970 \mathrm{~m}$ \\
\hline 77 & & 우 & $"$ & 11 & $"$ & $\because$ & $"$ \\
\hline 78 & & 우 & $"$ & $"$ & $"$ & $"$ & $\because$ \\
\hline 79 & \multirow{3}{*}{$\begin{array}{r}192162 \\
\text { PHALANGER } \mathrm{V} . \\
193097 \\
\text { SYCONYCTERIS } \\
\text { PIPISTRELLUS }\end{array}$} & $?$ & $(")>$ & $"$ & $"$ & $"$ & c. " \\
\hline 16780 & & EMB. & $"$ & $"$ & $"$ & $\because$ & $" 1$ \\
\hline 81 & & क & Oct. 2 & " & $"$ & $"$ & $\because$ \\
\hline 82 & \multirow{2}{*}{ MELOMYS F.? } & q & $"$ & $"$ & $"$ & $"$ & c. $\cdot$ \\
\hline 83 & & ๘ & $"$ & $"$ & $"$ & $"$ & c. . \\
\hline 84 & \multirow{2}{*}{$\begin{array}{l}\text { RaTtUS EXULANS } \\
\text { WALLABY } \\
\text { Wal }\end{array}$} & $\delta$ & " & $" 1$ & $"$ & " & $1970 \mathrm{M}$ \\
\hline 16785 & & $?$ & $(n)$ & $"$ & $"$ & 11 & c. " \\
\hline 86 & \multirow{2}{*}{$\begin{array}{c}\text { BANDICOOT } \\
\text { " } 190988 \\
0\end{array}$} & $?$ & OCT. 1 & , & $"$ & $"$ & $"$ \\
\hline 87 & & $?$ & Oct. 2 & $"$ & $"$ & $"$ & $"$ \\
\hline 88 & \multirow{4}{*}{$\begin{array}{r}190876 \\
\text { ANTECHINUS } \\
192927 \\
\text { PIPISTRELLUS } \\
192953 \\
\text { MINIOPTERUS (L) } \\
\text { "192954 }\end{array}$} & $\delta$ & Oct 3 & \multicolumn{3}{|c|}{ : KRINANTU AREa; Si nOFi } & c. $6200 \mathrm{FT}$. \\
\hline 89 & & $d$ & Oct. 2 & \multicolumn{3}{|c|}{ : OKAPA AREF; PUROSA } & $1970 \mathrm{M}$ \\
\hline 16790 & & & Oct 3 & \multicolumn{3}{|c|}{ : KAINANTU AREA; Si nOFi } & c. $6200 \mathrm{FT}$. \\
\hline 91 & & & $"$ & $"$ & 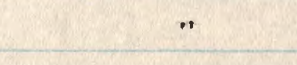 & " & 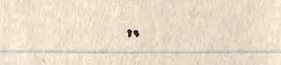 \\
\hline 92 & " 192955 & & $"$ & $"$ & " & $"$ & " \\
\hline 93 & \multicolumn{2}{|l|}{$\begin{array}{c}192999 \\
\text { MinIOPTERUS (s) } \\
193000\end{array}$} & " & $"$ & $"$ & $\ddot{*}$ & $"$ \\
\hline 94 & \multirow{3}{*}{$\begin{array}{ll}.193001 \\
. & 193002\end{array}$} & & $"$ & $"$ & * & “ & $"$ \\
\hline 16795 & & & $"$ & $" 1$ & .. & " & " \\
\hline 96 & & & $"$ & $"$ & $"$ & $"$ & “ \\
\hline 97 & \multirow{4}{*}{ 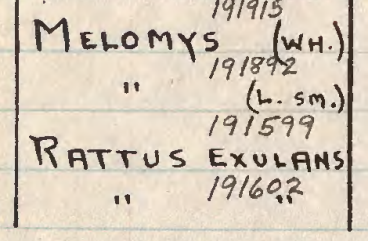 } & 와 & Oct. 6 & $"$ & Kratite Mts. & Arau & $1400 \mathrm{M}$ \\
\hline 98 & & 우 & $"$ & $"$ & " & $"$ & $"$ \\
\hline 99 & & 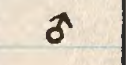 & $"$ & $"$ & $\cdot$ & $"$ & $"$ \\
\hline 6800 & & & $"$ & 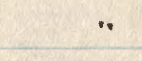 & $"$ & $"$ & $"$ \\
\hline
\end{tabular}


(60)

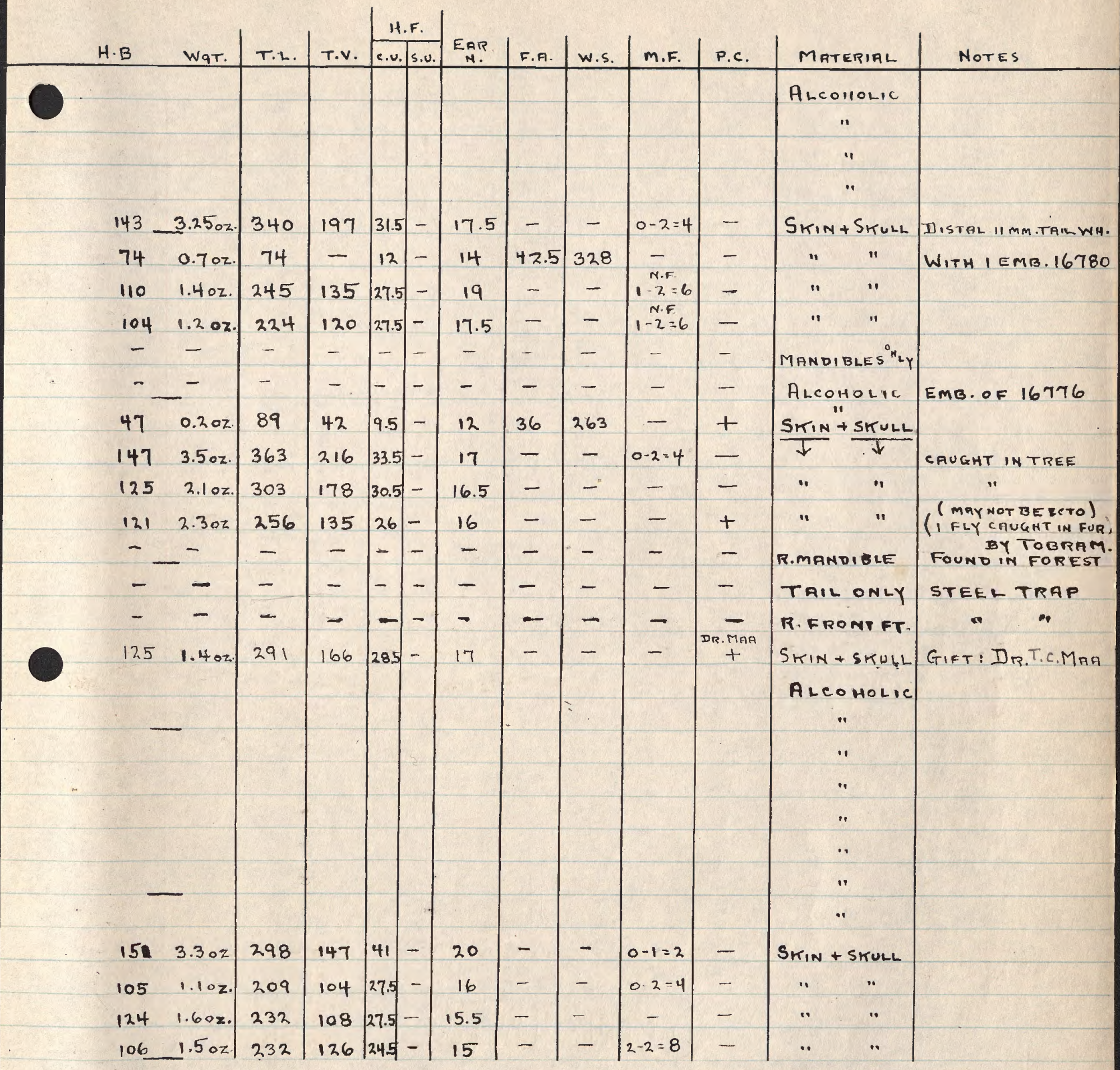




(61)

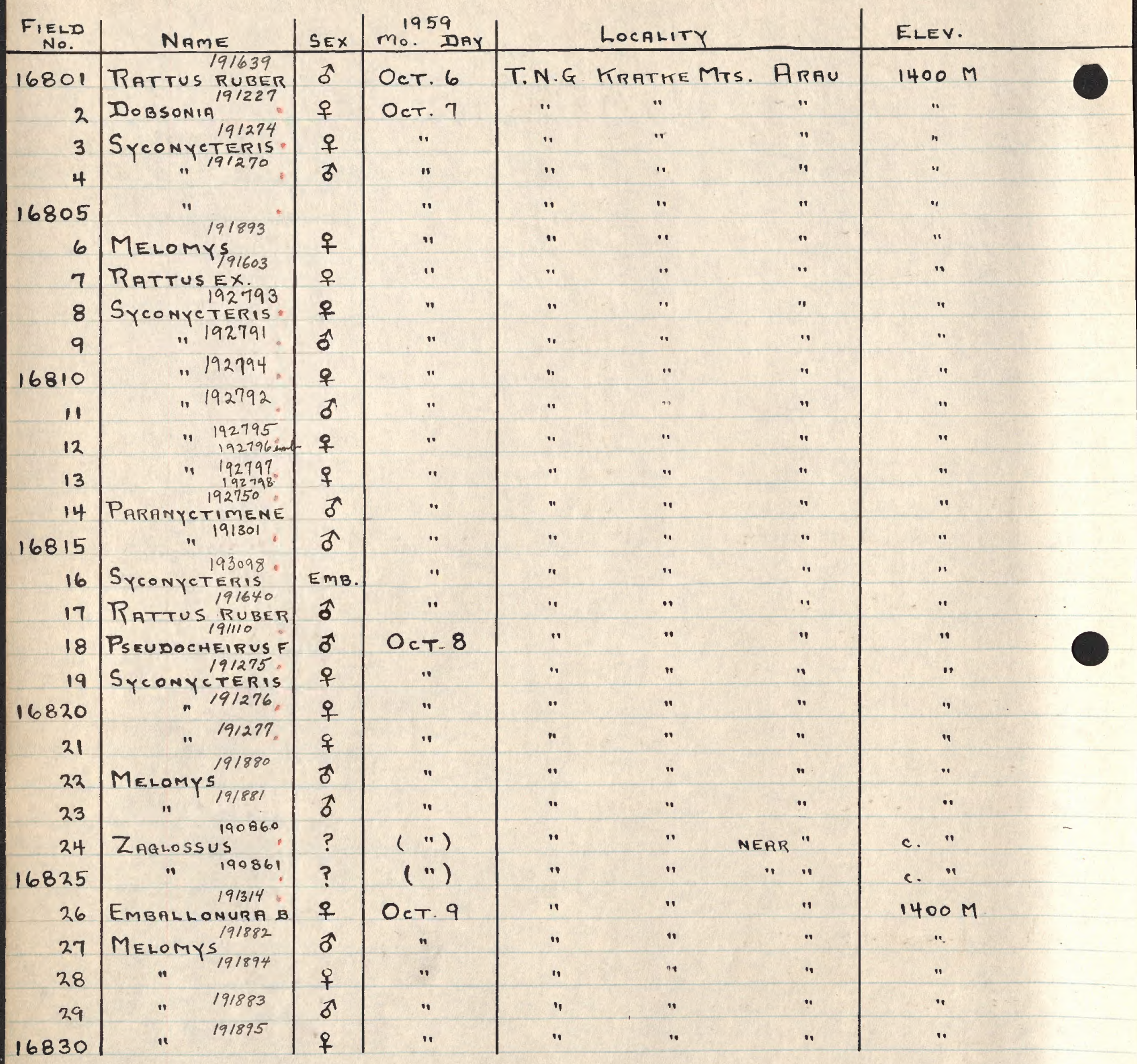

1830 


\begin{tabular}{|c|c|c|c|c|c|c|c|c|c|c|c|c|}
\hline \multirow[b]{2}{*}{$H \cdot B$} & \multirow[b]{2}{*}{ WaT. } & \multirow[b]{2}{*}{ T.L. } & \multirow[b]{2}{*}{ T.V. } & \multicolumn{2}{|c|}{ H.F. } & \multirow{2}{*}{$\begin{array}{l}\text { EAR } \\
N\end{array}$} & \multirow[b]{2}{*}{ F.A. } & \multirow[b]{2}{*}{ w.s. } & \multirow[b]{2}{*}{ M.F. } & \multirow[b]{2}{*}{ P.C. } & \multirow[b]{2}{*}{ MATERIAL } & \multirow[b]{2}{*}{ NotES } \\
\hline & & & & c.u. & s.u. & & & & & & & \\
\hline 140 & 3.007 & 267 & 127 & 32.5 & - & 18 & - & - & - & - & Skin + SkULL & \\
\hline 254 & $15.70 z$ & 284 & 30 & 42 & - & 30 & 150 & 1060 & $1-0=2$ & + & $" 11$ & \\
\hline 75 & 0.902 & 75 & - & 11.5 & - & 14 & 42.5 & 326 & - & - & " & $\begin{array}{l}\text { WITH EMB. } 16816 \\
\text { NETTED IN FICUS }\end{array}$ \\
\hline 78 & $0.70 z$ & 78 & - & 12 & - & 14 & 44 & 345 & - & - & $" \quad "$ & " \\
\hline & & & & & & & 44 & & & & Aleomolic & $"$ \\
\hline 108 & $1.50 z$ & 223 & 115 & 27.5 & - & 16 & 一 & - & $0-2=4$ & + & SKIN + SKULL & $\therefore$ \\
\hline 108 & $1.40 z$ & 226 & 118 & 25 & - & 15 & - & - & $2-2=8$ & - & " & \\
\hline & & & & & & & 41 & & & & AlcoholiC & NETTED IN Ficus \\
\hline & & & & & & & 41.5 & & & & $"$ & 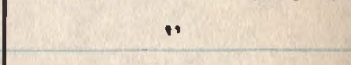 \\
\hline & & & & & & & 41 & & & & - & $"$ \\
\hline & & & & & & & 43 & & & & * & $"$ \\
\hline & & & & & & & 43.5 & & & & " & $\begin{array}{l}\text { WITH } \\
\text { EMB. }\end{array}$ \\
\hline & & & & & & & 44.5 & & & & " & - WITH \\
\hline 89 & $1.10 z$ & 110 & 21 & 15 & & 11.5 & 54 & 405 & - & - & " & $"$ \\
\hline 82 & $-1.10 z$. & 104 & 22 & 14 & - & 10.5 & 53.5 & 396 & - & - & SKIN + SKULL & THUMO $250 \mathrm{~mm}$. \\
\hline - & - & - & - & - & - & - & - & - & - & - & AlCOHOLIC & EMB.OF 16803 \\
\hline 152 & $3.8 \circ \mathrm{z}$ & 278 & 126 & 31.5 & - & 18 & - & - & - & + & SKIN+SKULL & \\
\hline 296 & $19.30 z$ & 592 & 296 & 42 & 38 & 20 & - & - & - & - & " & \\
\hline 73 & $0.50 z$ & 73 & - & 11.5 & - & 13 & 42.5 & 323 & - & - & $\ddot{*}=$ & NETTED IN FICUS. \\
\hline 72 & $-0.50 z$ & 72 & - & 12 & - & 12 & 42.5 & 321 & - & - & $"$ & \\
\hline 73 & $0.60 z$ & 73 & - & 12.5 & - & 13.5 & 43.0 & 332 & - & - & $"$ & \\
\hline 112 & $1.7 \mathrm{oz}$ & 233 & $\begin{array}{l}121 \\
\text { STUB }\end{array}$ & 28.5 & - & 16 & - & - & - & - & SKIN + SKULL & 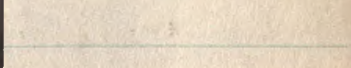 \\
\hline 111 & $1.60 \mathrm{z}$ & $(186)$ & $(75)$ & 28,5 & - & 16 & - & - & - & - & 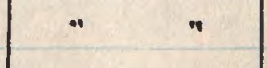 & \\
\hline - & - & - & - & - & - & - & - & - & - & - & STULL (PRRTIAL) & \\
\hline - & - & - & - & - & - & - & - & - & - & - & " & \\
\hline & $0.150 z$ & 60 & $(22) ?$ & 7 & - & 12 & 39.5 & $(272)$ & $1-0=2$ & - & Srin + SkULL & \\
\hline 115 & $1.60 z$ & $(157)$ & $\begin{array}{l}\text { STUB } \\
(42)\end{array}$ & 28.5 & - & 16 & - & - & - & $+\gamma^{\prime F}$ & " & \\
\hline 117 & $1.7 \mathrm{oz}$ & 235 & 118 & 28 & - & 16.5 & - & - & $0.2=4$ & $+\}$ & 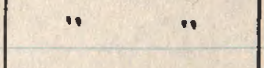 & \\
\hline 101 & $1.20 z$ & 222 & 121 & 30 & - & 16 & - & - & - & + & $"$ & \\
\hline$(99)$ & $1.1 \mathrm{oz}$. & $|(200)|$ & 101 & $27 \mid$ & $1-$ & 15.5 & - & - & - & +1 & 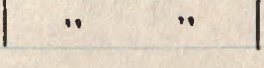 & RAC \\
\hline
\end{tabular}




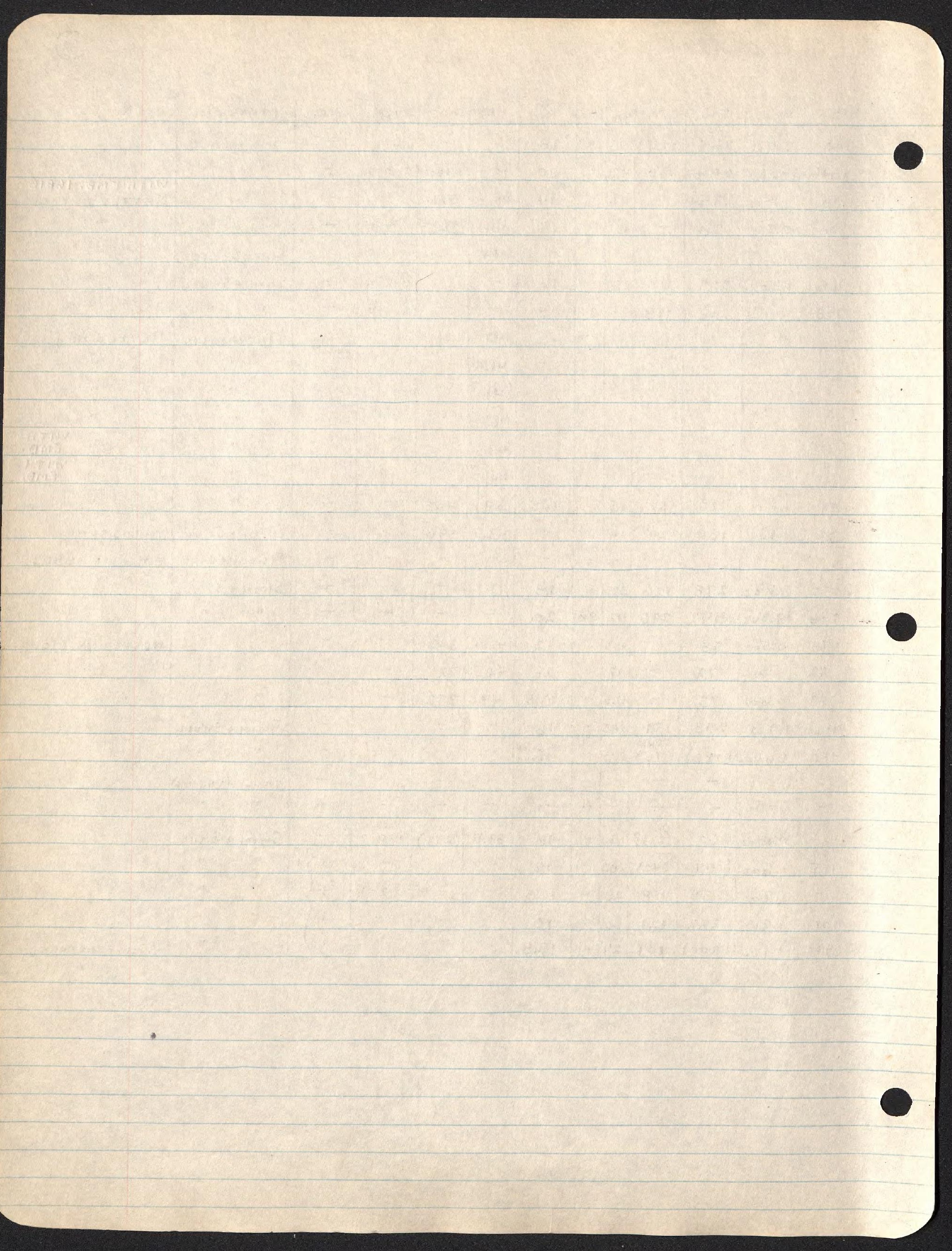



(62)

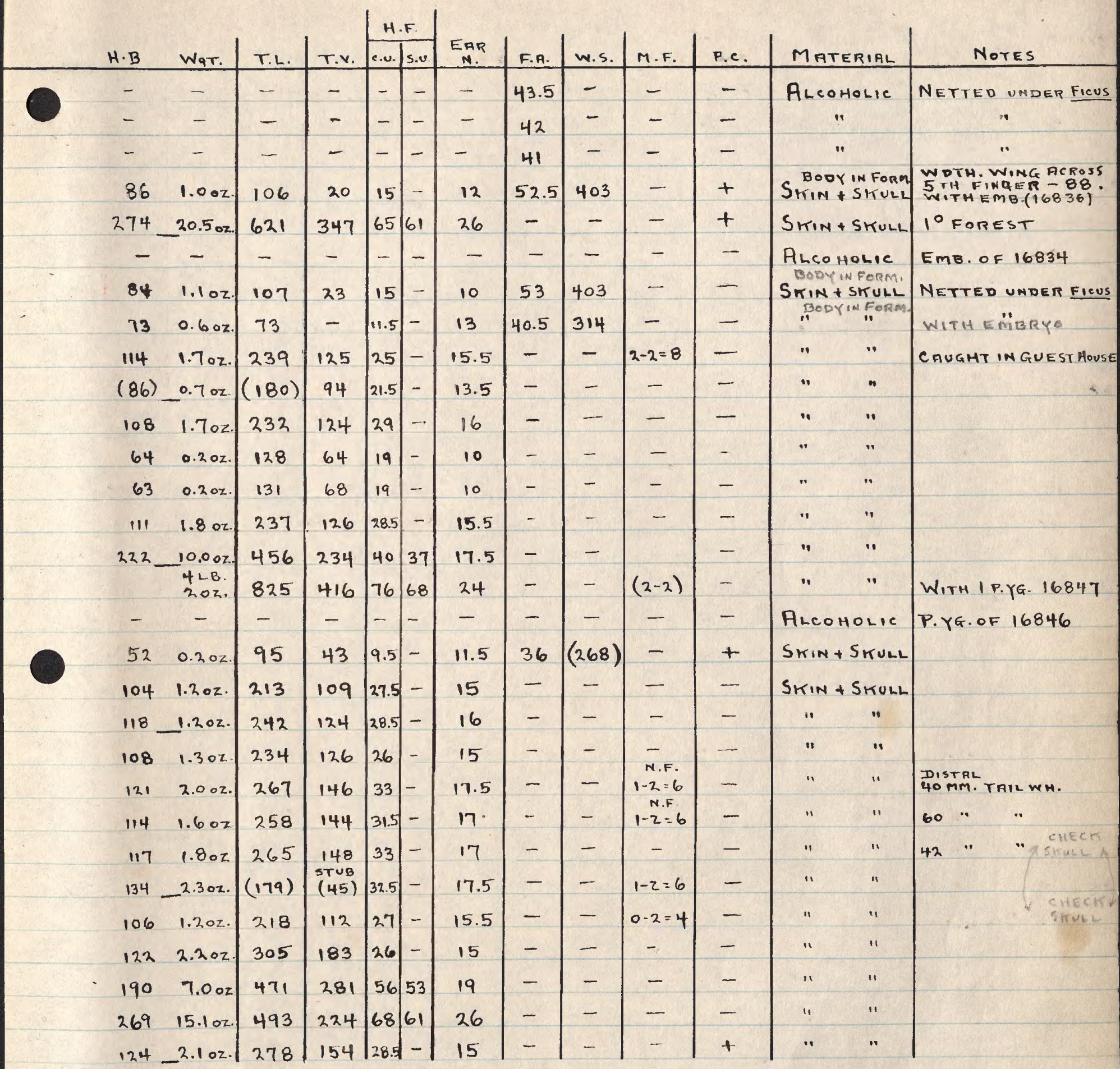



(63)

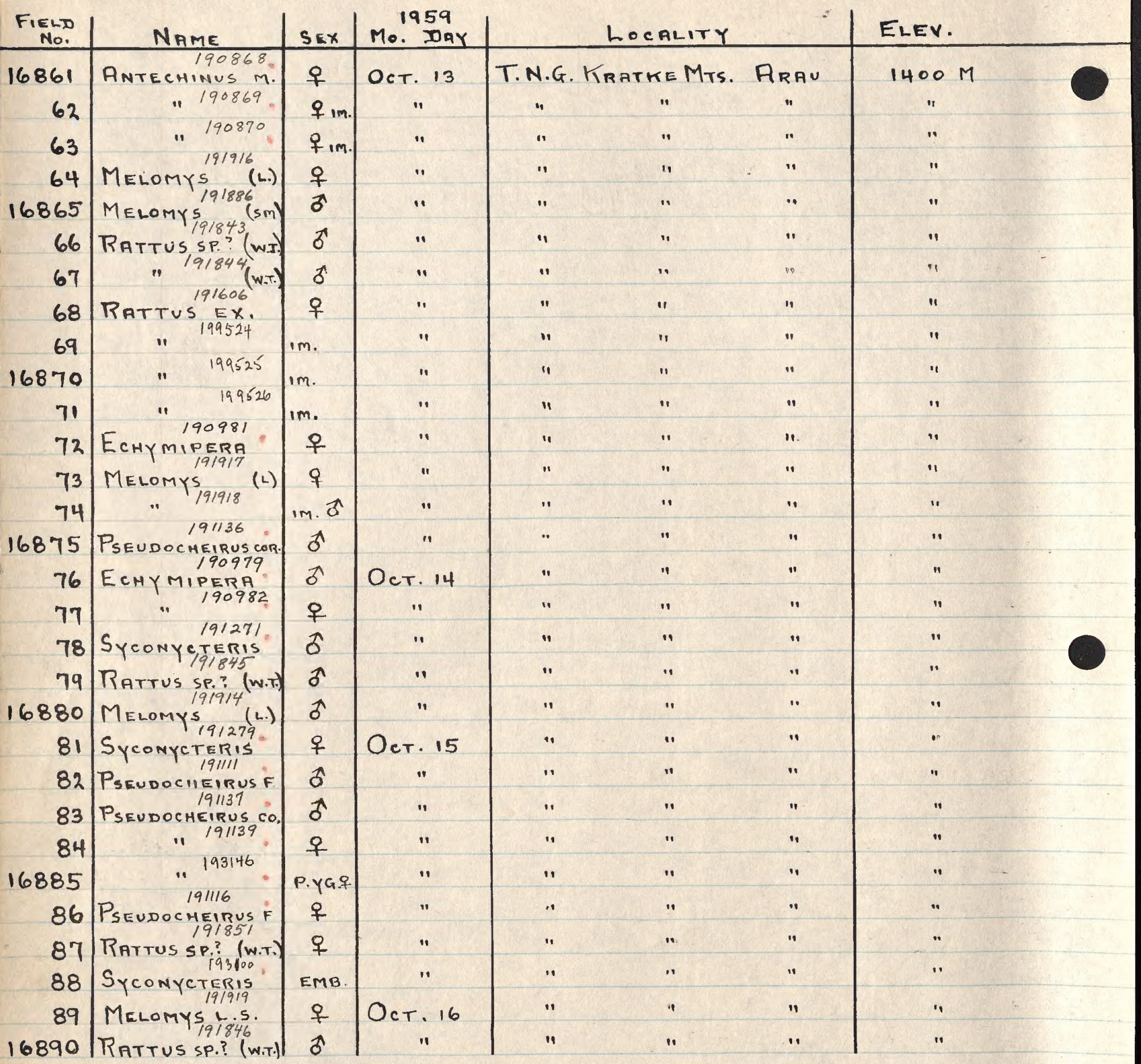

No $16886(191116)$ - SEE G.P.HOLLAND $(1969)$, FLEAS OF N.G., P. 10 
(63)

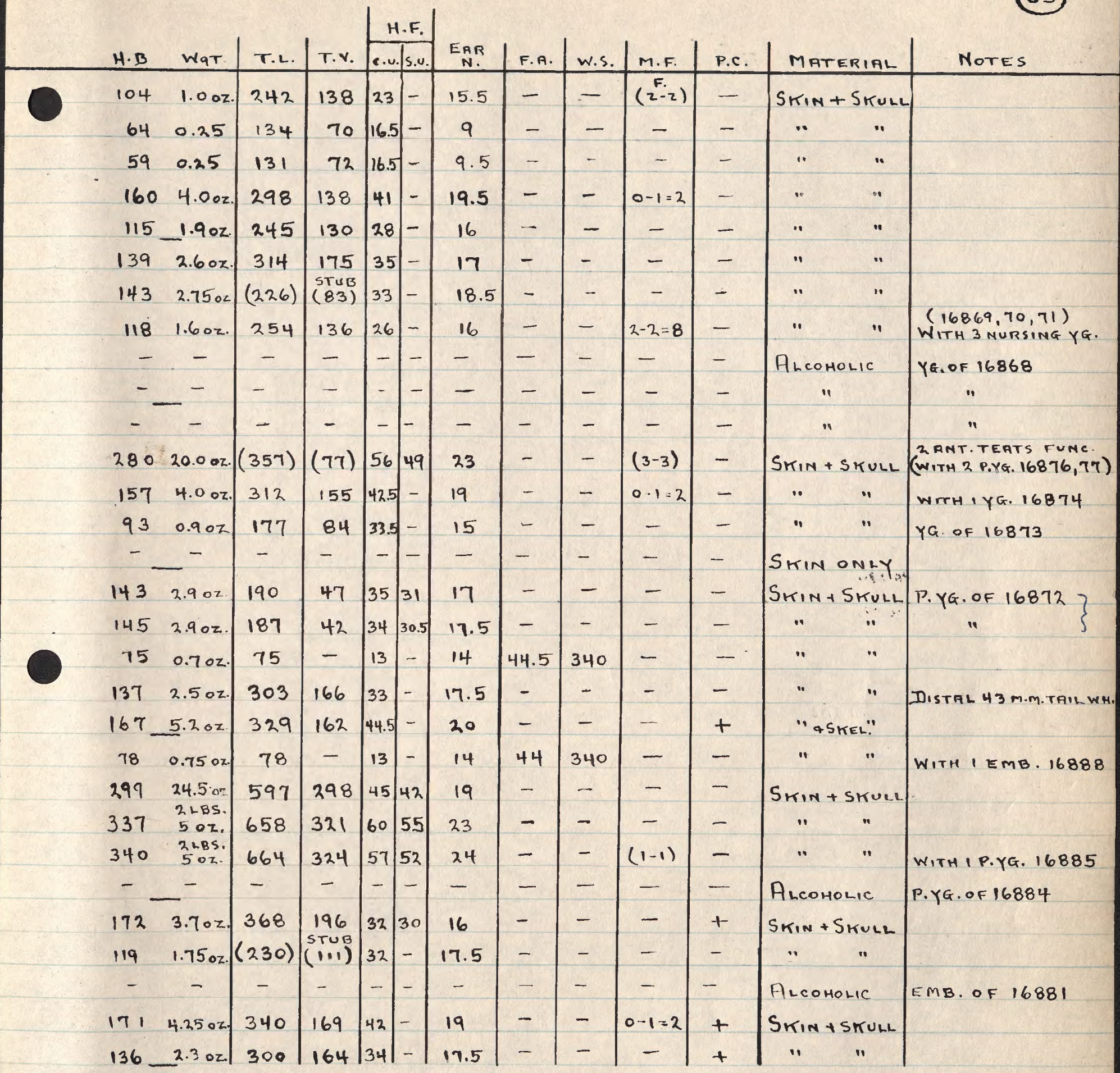




(64)

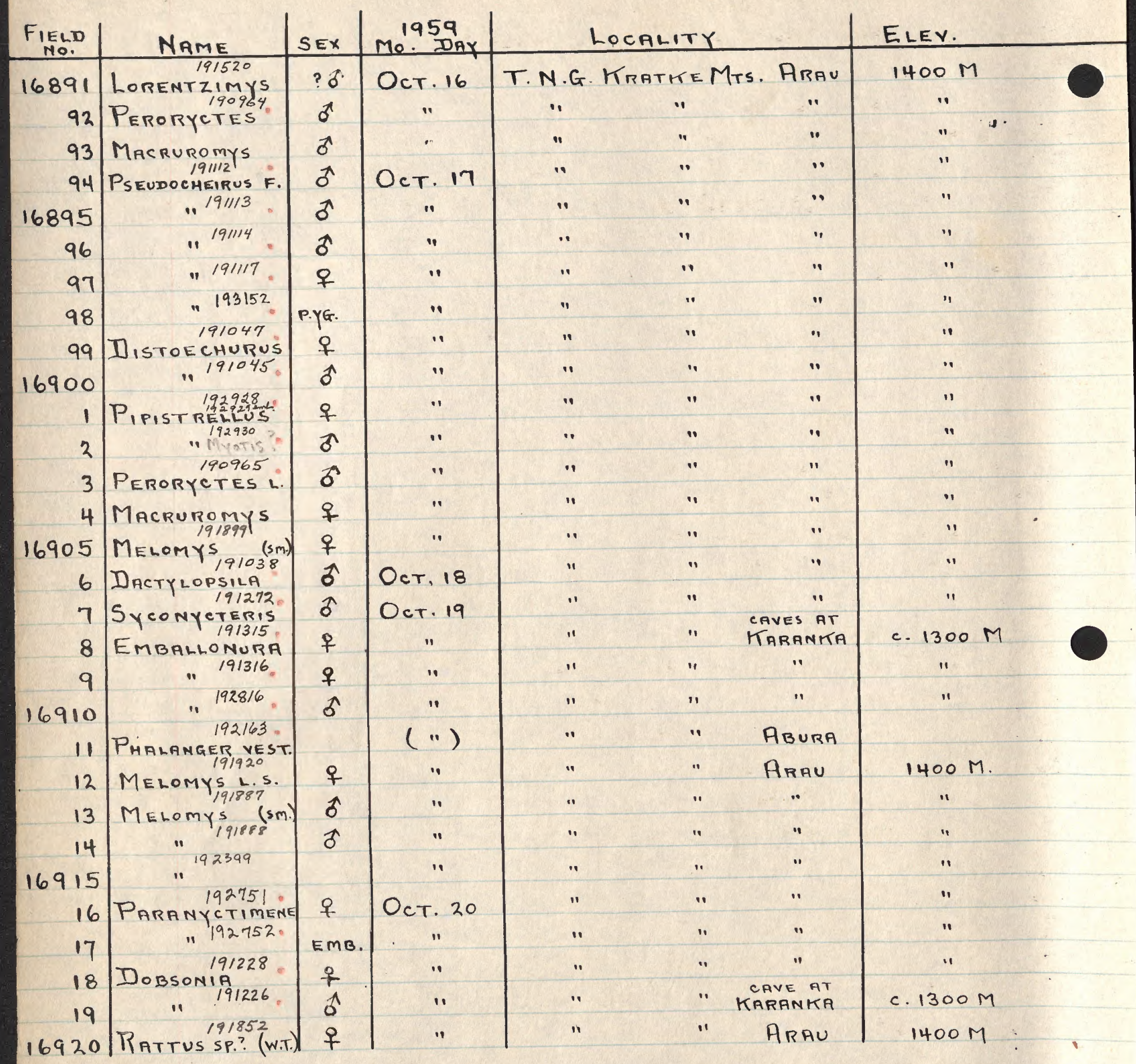

1920 
(64)

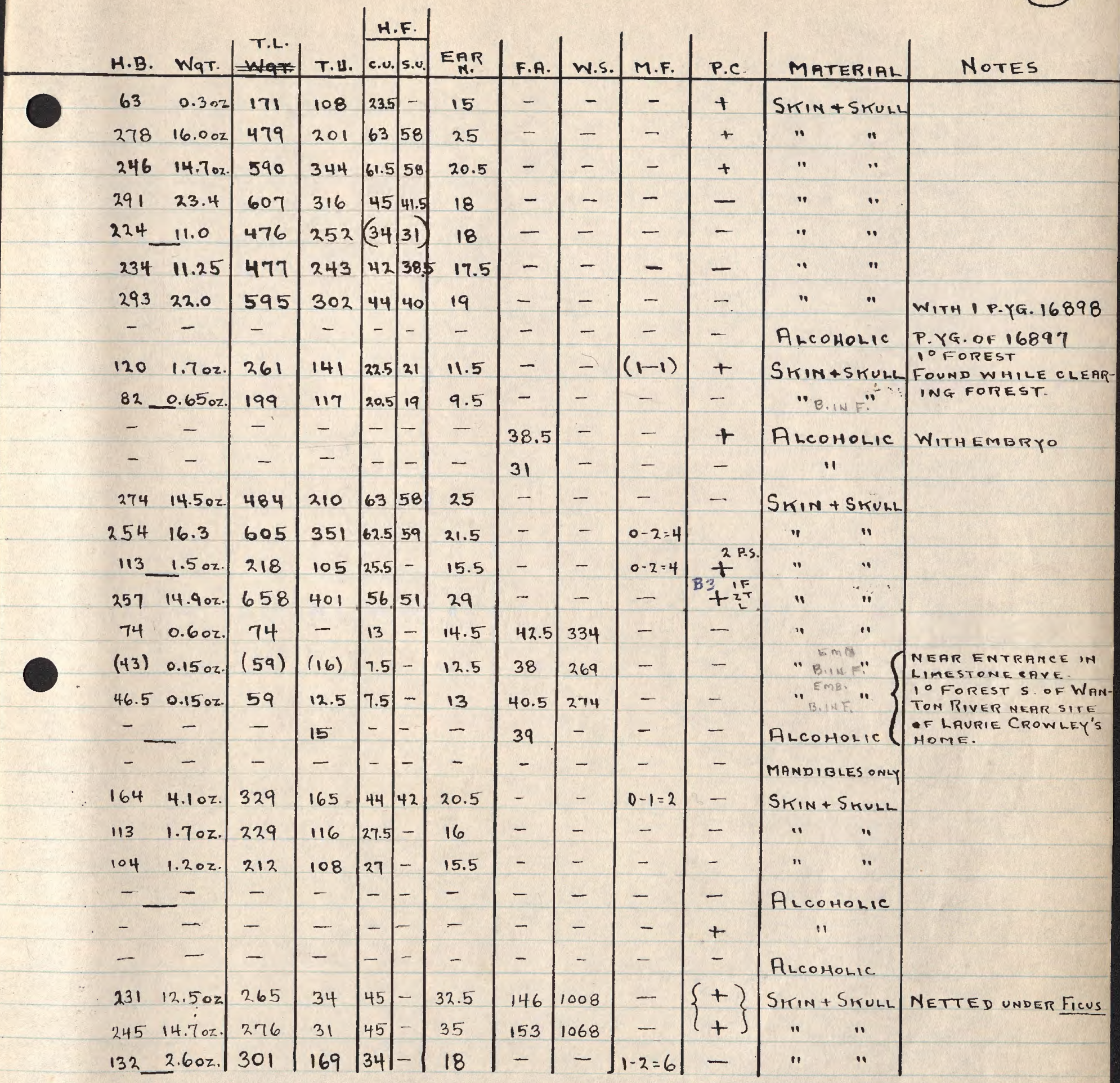


-

○ 



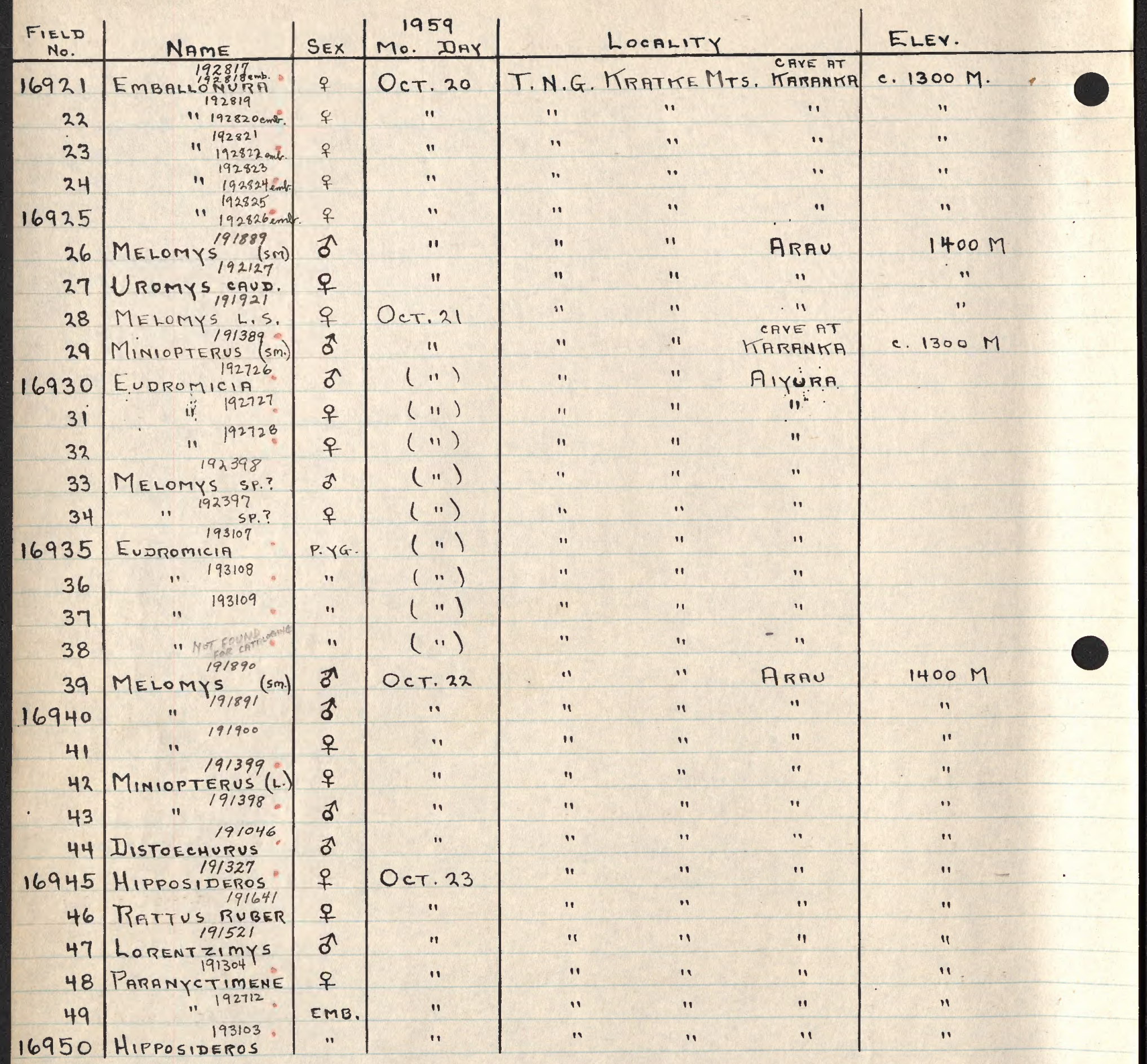


(65)

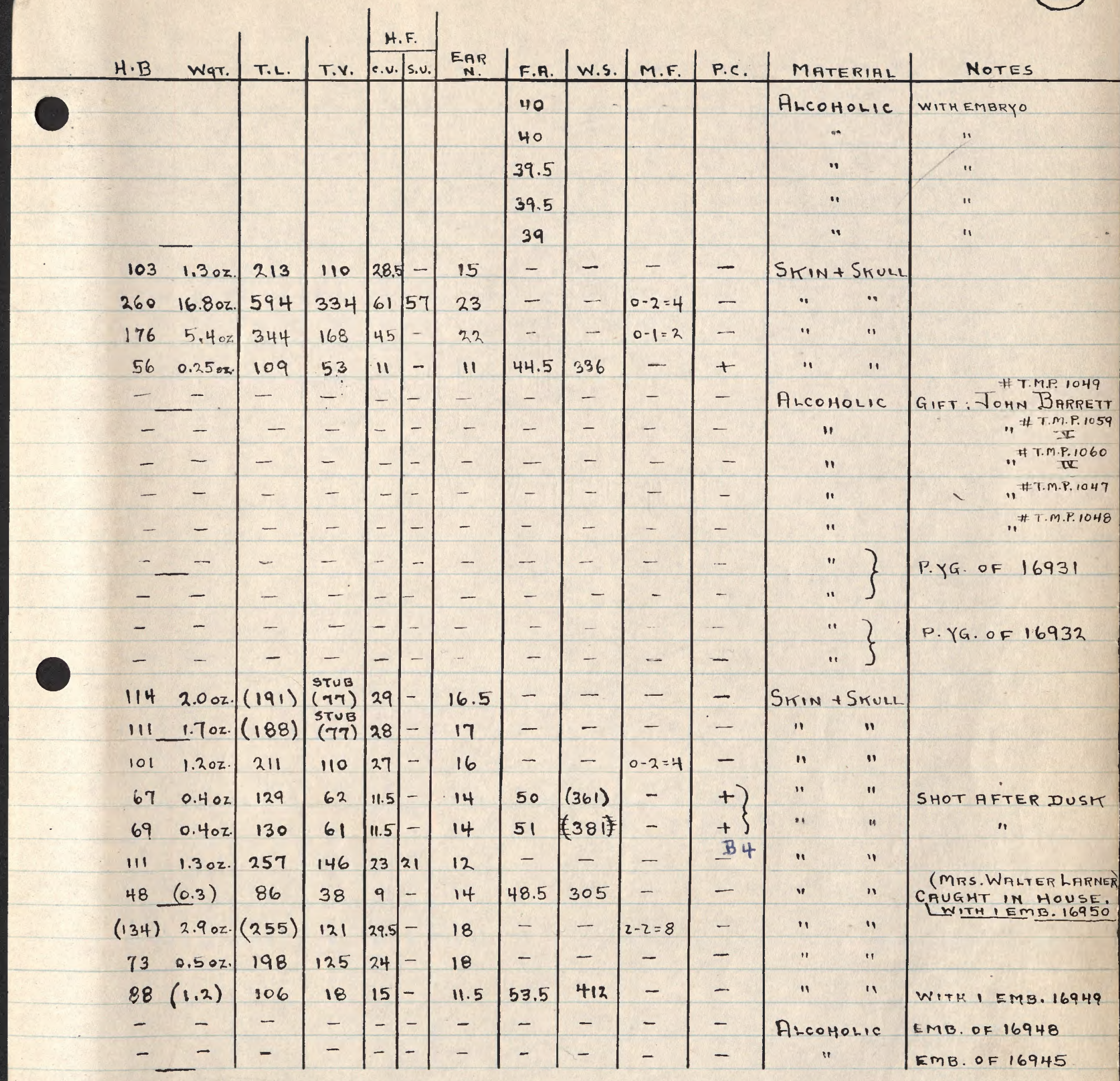






\begin{tabular}{|c|c|c|c|c|c|c|c|}
\hline $\begin{array}{c}\text { FIELD } \\
\text { No. }\end{array}$ & NAME & SEX & $\begin{array}{l}1959 \\
\text { Mo. DAY }\end{array}$ & & LOCALITY & & ELEX. \\
\hline 16951 & $\begin{array}{l}192128 \\
\text { UROMYS CAUD. }\end{array}$ & ㅇ․ & Oct. 24 & $T \cdot N \cdot G$. & KRATKE & Mts. Arau & $1400 \mathrm{M}$. \\
\hline 52 & MINIOPTERUS (L) & $\hat{\sigma}$ & " & $"$ & $"$ & $"$ & " \\
\hline 53 & ECHYMIPERA & $\operatorname{lm} . \approx$ & " & " & " & $\because$ & $\because$ \\
\hline 54 & PERORYCTES L.0. & 오 & " & " & " & $"$ & $"$ \\
\hline 16955 & Rattus sp.? (w.T.) & $\delta$ & $"$ & $"$ & " & " & $"$ \\
\hline 56 & RatTus(EX. Y) & $q$ & $"$ & $"$ & $"$ & " & $"$ \\
\hline 57 & PSEUDOCHEIRUS CO. & ๘ & " & " & " & " & $"$ \\
\hline 58 & Rousetrus & 오 & OCT. 25 & $"$ & $"$ & " & $" 1$ \\
\hline 59 & 192710 & EMB. & " & $"$ & " & $"$ & $" 1$ \\
\hline 16960 & TATTUS (WH.T) & 우 & $\because$ & "1 & $"$ & $\because$ & $" 1$ \\
\hline 61 & Rattus EX. & $\hat{3}$ & $"$ & " & $" 1$ & $"$ & 11 \\
\hline 62 & DACTYLOPSILA & $\widehat{\sigma}$ & Oct. 26 & " & $"$ & "ORHAYNA" & c. " " \\
\hline 63 & HYOROMYS & 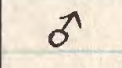 & $" 1$ & $\because$ & $"$ & NR. SASAR̈R & c. " \\
\hline 64 & EMBALLONURA & $q$ & OCT. 27 & $"$ & $"$ & KaSSAM & $1350 \mathrm{M}$ \\
\hline 16965 & $\therefore \quad 119282826$ eims & 우 & OCT. 28 & $" 1$ & $"$ & $"$ & $"$ \\
\hline 66 & PSEUDOCHEIRUS & $\hat{\sigma}$ & $"$ & $"$ & $" 1$ & $"$ & $"$ \\
\hline 67 & MELOMYS $(\mathrm{sm})$. & 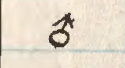 & " & $"$ & $"$ & " & " \\
\hline 68 & $1119 / 902$ & A & $"$ & $"$ & " & $"$ & $"$ \\
\hline 69 & " 191907. & $q$ & " & $"$ & " & " & $"$ \\
\hline 16970 & $11 \quad 191908$ " & $q$ & $"$ & $"$ & $"$ & " & 11 \\
\hline 71 & PERORYCTES RRF. & $q$. & $" 1$ & $"$ & $"$ & $"$ & $" 1$ \\
\hline 72 & DACTYLEPSILA & $\delta$ & " & " & " & $"$ & 11 \\
\hline 73 & $\begin{array}{l}19 / 317 \\
\text { EMBALLONURA }\end{array}$ & $\hat{\sigma}$ & OCT. 29 & $"$ & $"$ & " & " \\
\hline 74 & PIPISTRELLUS & q & $"$ & " & " & $"$ & " \\
\hline 16975 & $\begin{array}{l}191292 . \\
\text { NYcTIMENE }\end{array}$ & $\hat{\sigma}$ & " & $"$ & $"$ & " & $"$ \\
\hline 76 & MELOMYS 191903 & $\hat{\sigma}$ & $"$ & " & " & ". & " \\
\hline 77 & 11192700 & $E M B$. & Oст. 28 & $"$ & $"$ & " & $" 1$ \\
\hline 78 & 192701 & EMB. & $"$ & ". & " & $"$ & $"$ \\
\hline 79 & EMBALLO'NSTR & f & Oct. 30 & $"$ & " & $"$ & " \\
\hline 16980 & $119283 !$ & $\because$ & 11 & $"$ & $" 1$ & $" 1$ & " \\
\hline
\end{tabular}

\section{Arau}

NO. SPECIMENS 158

NO. SPECIES
30
Aiyura

9

2
TOTALS 167

31 


\begin{tabular}{|c|c|c|c|c|c|c|c|c|c|c|c|c|}
\hline$H \cdot B$ & WqT. & T.L. & T.V. & c.u. & s.u. & $\begin{array}{l}E A R \\
N \text {. }\end{array}$ & F.A. & W.S. & M.F. & P.C. & MATERIAL & NOTES \\
\hline 208 & $9.00 \mathrm{z}$ & 494 & 286 & 57.5 & 54 & 23 & - & - & $0-2=4$ & ${ }^{B 2}+1 F$ & SKIN + SKULL & \\
\hline- & - & - & - & - & - & - & 52 & - & - & + & AlcoHOLIC & \\
\hline 132 & $1.60 z$ & 176 & 44 & 32. & 28.5 & 17 & - & - & - & - & SKIN + SKULL & \\
\hline 270 & $14.30 z$ & 457 & 187 & 60 & 55 & 25 & - & - & $(z-2)$ & - & " & $\begin{array}{l}\text { NO POUCH YG. PRESENT } \\
\text { RIGHT ANT. TEAT FUNC. }\end{array}$ \\
\hline 120 & $-1.70 z$ & 273 & 153 & 33 & 31.5 & 17.5 & - & - & - & - & $"$ & DISTAL $51 \mathrm{mM}$. TAIL WHITE \\
\hline 96 & $1.00 z$ & $(186)$ & $(90)^{B}$ & 24 & - & 15.5 & - & - & - & - & $"$ & \\
\hline 268 & 16.7 & 554 & 286 & 51 & 47 & 22 & - & - & - & + & $"$ & \\
\hline 135 & $(3.3)$ & 151 & 16 & 23 & - & 20 & 87 & 591 & $1-0=2$ & + & " & $\begin{array}{l}\text { INGROVE OF FICUS } \\
\text { CAUGHT IN MIST NET. } \\
\text { RAIN + CLOUDS. }\end{array}$ \\
\hline - & - & - & - & - & - & - & - & - & - & - & AlCOHOLIC & EMB.OF 16958 \\
\hline 116 & $1.80 z$ & 278 & 162 & 32.5 & - & 17 & - & - & - & - & SKIN + SKULL & \\
\hline 96 & $1.20 \mathrm{z}$ & 225 & 129 & 25 & - & 15 & - & - & - & - & 11 & \\
\hline 247 & $160 z$ & 627 & 380 & 58 & 53 & 29 & - & - & - & - & $"$ & HOLE IN FELLED TREE \\
\hline 239 & $11.90 z$ & 489 & 250 & 58 & 56 & 17.5 & - & - & - & $一$ & " & ROCKY MT. BROOK \\
\hline 42 & $0.10 z$ & 54 & 12 & 7.5 & $1-$ & 13 & 39 & $(265)$ & - & - & " & $\begin{array}{l}\text { FOREST. } \\
\text { SHOT. CLEARING IN }\end{array}$ \\
\hline- & - & - & - & - & - & - & & - & - & - & ALCOHOLIC & "(WITH Em"BRYO) \\
\hline $27^{\circ}$ & $18.60 z$ & 555 & 285 & 45 & 41 & 19 & - & - & - & - & SKIN + SKULL & \\
\hline 115 & $1.90 z$ & 242 & 127 & 30 & - & 16.5 & - & - & - & + & $"$ & \\
\hline 111 & $1.60 z$ & $(207)$ & $(96)$ & 29 & - & 16 & - & - & - & + & $" 1$ & \\
\hline 108 & $1.4 \mathrm{oz}$ & 228 & 120 & 27.5 & - & 16 & - & - & $0-2=4$ & + & "BODX" & \\
\hline 111 & $1.90 \mathrm{z}$ & 227 & 116 & 27.5 & - & 15.5 & - & - & $\begin{array}{r}\text { FUNC. } \\
0-2=4\end{array}$ & + & $\begin{array}{l}\text { "BOBY "IN } \\
\text { ALC. }\end{array}$ & $\begin{array}{l}\text { WITH } 2 \text { EMBRYOS : } \\
16977 \text { - 16978 } \\
\text { VENTRAL VIEW }\end{array}$ \\
\hline 345 & $25.30 z$ & 529 & 184 & 79 & 75 & 32 & - & - & $(4-4)$ & - & $" 1$ & ANT. :: : POST. \\
\hline 267 & $16.40 z$ & 641 & 374 & 55 & 50 & 28 & 一 & - & - & - & $"$ & \\
\hline & $0.10 z$ & 55 & 15 & 7.5 & - & 13 & 38.5 & 265 & - & + & " & \\
\hline 51 & $0.20 z$ & 84 & 33 & 7.5 & - & 11 & 35 & 260 & - & $\begin{array}{l}\text { DR.T.C.MAF } \\
(+1)\end{array}$ & "BooY " & $\begin{array}{l}192937 \\
\text { WITHEMBRY }\end{array}$ \\
\hline 94 & $1.250 z$ & 122 & 28 & 14 & - & 13 & 58 & 444 & - & $\$$ & SKIN+SKULL & NETTED \\
\hline 117 & 1.75 oz. & 247 & 130 & 29 & - & 16.5 & - & - & - & + & " & \\
\hline - & - & - & - & - & - & - & - & - & - & - & AncoHOLIC & EMB. OF 16970 \\
\hline - & - & - & - & - & - & - & - & - & - & - & 11 & 1 \\
\hline - & - & - & - & - & - & - & 38 & - & - & $(+)\}^{T . C . M A P}$ & $" 1$ & WITH EMBRYO \\
\hline - & - & - & 一 & - & - & - & 39.5 & - & - & $(t)\}$ & $"$ & \\
\hline
\end{tabular}






\begin{tabular}{|c|c|c|c|c|c|c|c|}
\hline $\begin{array}{c}\text { FIELD } \\
\text { No. }\end{array}$ & NAME & SEX & $M_{0}^{1959}$ DAY $^{19}$ & \multicolumn{3}{|c|}{ LOCALITY } & ELEY. \\
\hline 16981 & MINIOPTERUS (L) & $f$ & Oct. 30 & T.N.C & KE & ASSAM & $1350 \mathrm{M}$ \\
\hline 82 & PSEUDOCHEIRUS' & $\hat{\sigma}$ & $" 1$ & 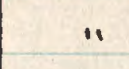 & $"$ & " & $"$ \\
\hline 83 & MELOMYS 191904 ( $5 \mathrm{r}$ ) & ๙ & $"$ & $" 1$ & $"$ & " & " \\
\hline 84 & Rattus Ex. & ठ & $"$ & $"$ & $" 1$ & 11 & $"$ \\
\hline 16985 & 191611 & $\hat{\sigma}$ & $"$ & $"$ & 1 & “ & " \\
\hline 86 & $\begin{array}{r}191522 \\
\text { LORENTZI MYYS }\end{array}$ & q & $" 1$ & 11 & 11 & " & " \\
\hline 87 & MINIOPTERUS (sm) & 우 & Oct.31 & $"$ & " & " & " \\
\hline 88 & 11 193003. & $\delta$ & 11 & $"$ & $"$ & $"$ & $"$ \\
\hline 89 & $\begin{aligned} & 193004 \\
& 11 \quad 193005_{\text {EMB. }}\end{aligned}$ & 우 & $"$ & $" 1$ & $"$ & $"$ & $"$ \\
\hline 16990 & PSEUDOCHEIRUS F. & ot & $" 1$ & $"$ & $\because$ & $"$ & 1 \\
\hline 91 & HYDROMYs 191433 & 우 & 11 & $" 1$ & $"$ & $" 1$ & $"$ \\
\hline 92 & Tattus EX. & $\delta$ & $"$ & $" 1$ & 11 & " & " \\
\hline 93 & " & q & $"$ & 11 & $" 1$ & $"$ & $" 1$ \\
\hline 94 & Hyorzomys & $\delta$ & " & "1 & 11 & $"$ & $\because$ \\
\hline 16995 & DOBSONIA & 우 & $"$ & $" 1$ & $" 1$ & " & " \\
\hline 96 & Rattus RUBER & $\hat{\sigma}$ & Nor. I & $"$ & " & $"$ & " \\
\hline 97 & RatTUS EXULANS & $q$ & "1 & 11 & $"$ & $"$ & $"$ \\
\hline 98 & 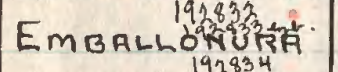 & $q$ & "1 & 11 & 11 & $" 1$ & " \\
\hline 99 & $\begin{array}{r}192834 \\
1192835 \\
10 \text { anth }\end{array}$ & $q$ & " & 11 & $" 1$ & $" 1$ & $"$ \\
\hline 17000 & $\begin{array}{l}191357 \\
\text { PHILETOR }\end{array}$ & 오 & " & $" 1$ & $" 1$ & 11 & $"$ \\
\hline 1 & PSEUDUCHEIRUS F. & $\sigma^{x}$ & $"$ & " & $"$ & "1 & $"$ \\
\hline 2 & 11191122 & $\delta$ & $" 1$ & $\because$ & $" 1$ & $"$ & $" 1$ \\
\hline 3 & Pogonomys (hs.) & $q$ & $"$ & 11 & $"$ & $"$ & $"$ \\
\hline 4 & 11 $191497^{\circ}$ & 우 & $"$ & $"$ & " & " & $"$ \\
\hline 17005 & $\because 19 / 498$ & $q$ & $"$ & $"$ & " & $"$ & $"$ \\
\hline 6 & 1191489 & $\hat{\sigma}$ & $"$ & $"$ & " & $\because$ & " \\
\hline 7 & Pogonomys (s.5) & $q$ & $"$ & $" 1$ & $"$ & " & " \\
\hline 8 & Rattus RUBER & $q$ & $"$ & $"$ & $"$ & " & $"$ \\
\hline 9 & MELOMYS & 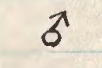 & $"$ & $"$ & " & $"$ & $"$ \\
\hline 17010 & 11191909 & $q$ & $"$ & $"$ & $"$ & " & " \\
\hline
\end{tabular}


\begin{tabular}{cc|c|c|c|c|c|c|c|c|c|c|c} 
H.B & WGT. & T.L. & T.V. & C.U. & S.U. & ER. & F.A. & W.S. & M.F. & P.C. & MATERIAL & NOTES
\end{tabular}

\begin{tabular}{ll|l|l|l}
70 & $0.60 \%$ & 127 & 57 & 12
\end{tabular}

$\begin{array}{lllllll}242 & 14.25 & 521 & 279 & 45 & 41\end{array}$

$\begin{array}{llllll}119 & 1.80 z & 220 & 101 & 29 & -\end{array}$

$114 \quad 1.60 z \quad 249 \quad 135 \quad 25.5$ -

(107) 1.302. $(234) \quad 127 \quad 27-$

\begin{tabular}{lll|l|l|}
76 & 0.702 & 207 & 131 & 25 \\
\hline
\end{tabular}

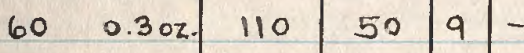

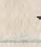

$$
-
$$

$\begin{array}{llllll}191 & 5.902 & 415 & 224 & 37.5 & 35\end{array}$

\begin{tabular}{ll|l|l|l}
225 & $10.70 z$ & 470 & 245 & 54.551
\end{tabular}

109 1.4oz. $240 \quad 131 \quad 27.5$ -

109 1.7oz. 242

$244 \quad 14.407 \quad 493$

$\begin{array}{lllllll}238 \quad 15.0 \text { oz. } & 266 & 28 & 45 & -\end{array}$

$150 \quad 3.60 z .(257)(107) 30$

\begin{tabular}{lll|l|l}
118 & 1.907 & 257 & 139 & 26
\end{tabular}

$18.5-5-$

$(+)$ SMIN + SKULL SHOT;IN CAR LIGHTS

\begin{tabular}{|c|c|c|c|}
\hline 16.5 & - & - & - \\
\hline 15 & - & - & - \\
\hline 15.5 & - & - & - \\
\hline
\end{tabular}

\begin{tabular}{cc|c|c|c|c}
- & - & - & - & - & - \\
60 & $0.40 z$ & 93 & 33 & 9 & - \\
261 & $16.00 z$ & 536 & 275 & 44 & 40 \\
208 & $8.30 z$ & 440 & 232 & 40 & 37 \\
141 & $3.50 z$ & 370 & 229 & 29 & - \\
138 & $2.60 z$ & 353 & 215 & 29.5 & - \\
139 & $3.00 z$ & 365 & 226 & 31 & - \\
116 & $1.70 z$ & 316 & 200 & 28 & - \\
137 & $3.10 z$ & 335 & 198 & 31.5 & - \\
120 & $1.90 z$ & 230 & 110 & 28 & - \\
113 & $1.50 z$ & 235 & 122 & 28.5 & - \\
111 & $1.30 z$ & 235 & 124 & 26.5 & -
\end{tabular}

18

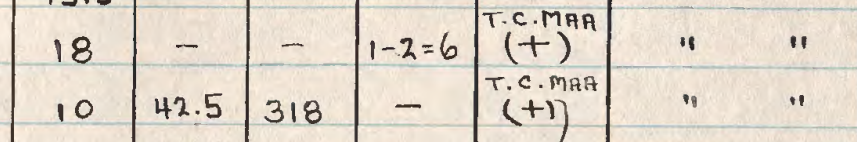

$-\begin{gathered}41.5 \\ 43\end{gathered}-\left[\begin{array}{c}(+) \\ (+)\end{array}\right\}$ Alcoholic 


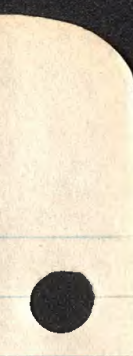



(68)

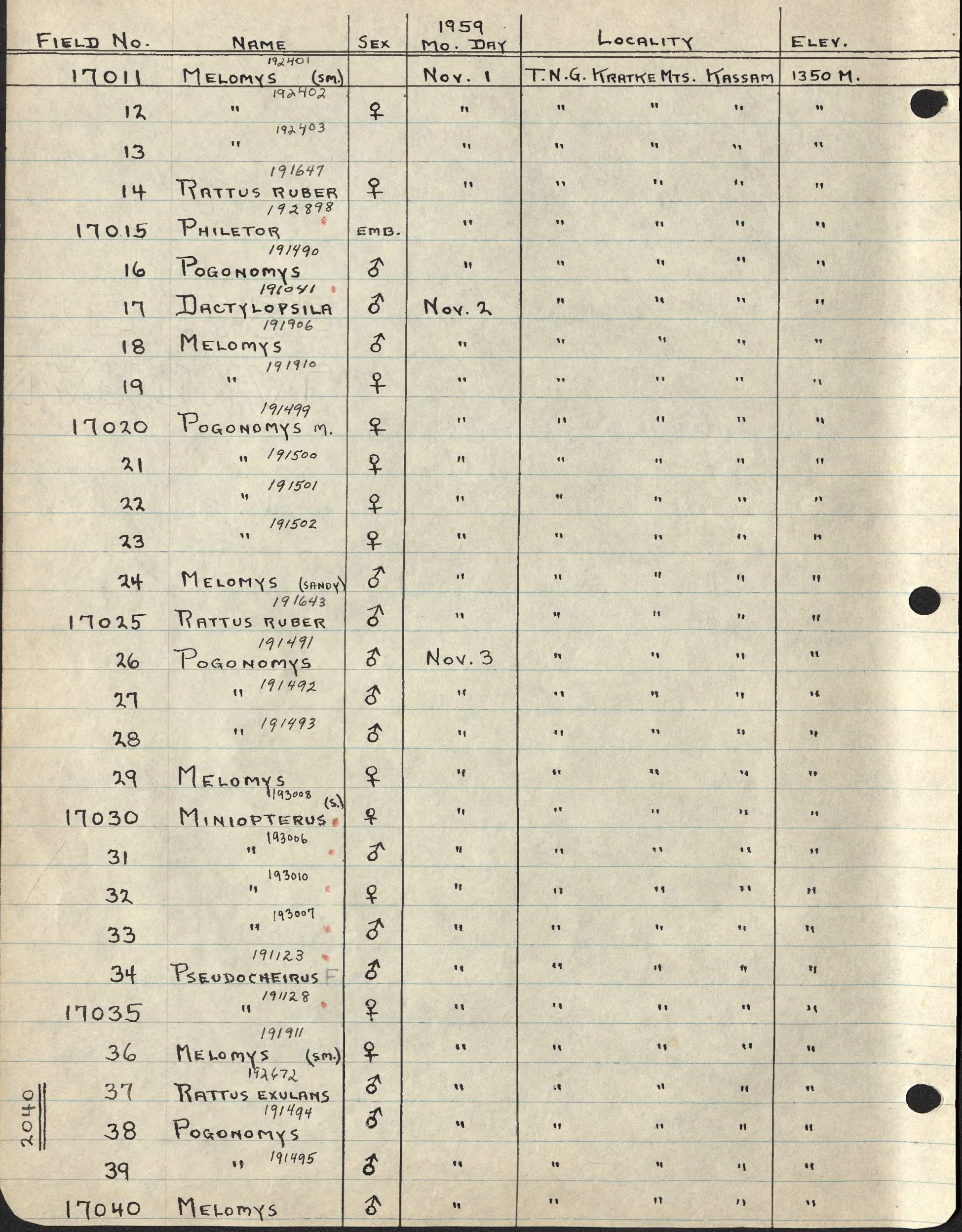




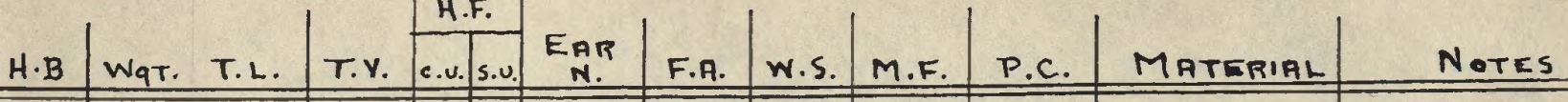

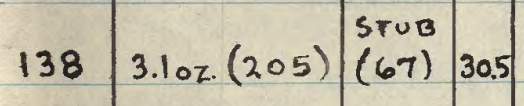

$1 1 6 \longdiv { 2 . 0 0 z . 2 9 9 }$

267 17.80r. 647

1131.9 \% . (199)

\begin{tabular}{lll|l|l|}
115 & 1.75 oz. 237 & 122 & 27.5
\end{tabular}

\begin{tabular}{lll|l|l}
110 & $1.70 z$ & 302 & 192 & 26.5 \\
\hline
\end{tabular}

$1333.00 z .335 \quad 202 \quad 27$

$\begin{array}{llllll}136 & 3.20 z . & 360 & 224 & 28.5 & \text { - }\end{array}$

\begin{tabular}{lll|l|l}
139 & $2.90 z .355$ & 216 & 29
\end{tabular}

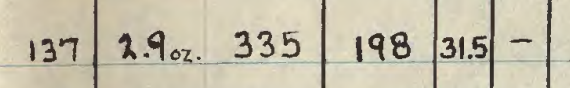

$141 / 3.302 .279 \quad 138 \quad 325-$

$124-332 \quad 20827-14$

$124-330206285-15$

$122-328 \quad 20627-15$

$123-278 \quad 15528.5-15$

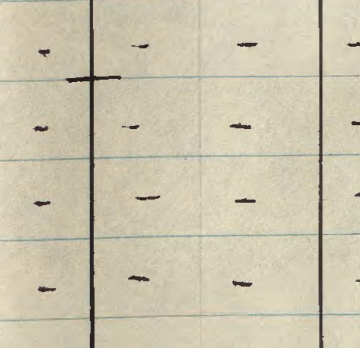

\begin{tabular}{lllll|l|l}
201 & $6.40 z$. & 418 & 217 & 37.5 & 35 & 17.5
\end{tabular}

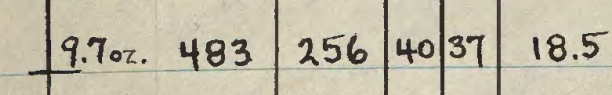

115 1.7oz. $237 \quad 122 \quad 27$

\begin{tabular}{c|cc|c|c}
132 & $3.10 z$ & 332 & 200 & 29.5 \\
110 & $1.50 z$ & 290 & 180 & $28.5-$ \\
133 & $2.80 z$ & 291 & 158 & 30.5
\end{tabular}

16

16

13

13.5

20

\begin{tabular}{|c|c|c|c|c|c|}
\hline - & - & - & - & ALCOHOLIC & \\
\hline - & - & - & - & $"$ & WITH 2 EMBRYOS \\
\hline - & - & - & - & " & \\
\hline - & - & $2-2=8$ & $\begin{array}{l}\text { T.c.m. } \\
(+)\end{array}$ & SKIN + SKULL & \\
\hline
\end{tabular}

- AlcohOLIC

T.c.M.

(t) 5 KIn + SKULL

t.c.m.
$(+)$

$(+)$

$14-1-2=6$

15.5

$-$

$-$

$-$

$-$

$-1-\{(+)\}$

$--\frac{\text { T.e.m. }}{(+)}$

$-\quad-0-2=4 \quad(t)$

42.5

T.c.M.

42.0

$40.5-$

$42.5-$

$(+)$

Alcomolic

TEATS $5 \mathrm{~mm}$. APART

193009
WITH EMBRYO

193011
WITH EMBRYo

$(+)$

$(+)$

T.c.m.

$-1-\quad S_{K I N}+S_{K U L L}$

$(1-1) \mid \begin{gathered}(+1) \\ \text { T.C.M. }\end{gathered}$

$0-2=4 \quad(+)$

T.c.M.

(t) AlCOHOLIC

T.c.m.

(H) SKIN + SKULL TAIL DAMAGED + HEALED

(+) $"$

T.c.m.

$(+)$ 
. 

(69)

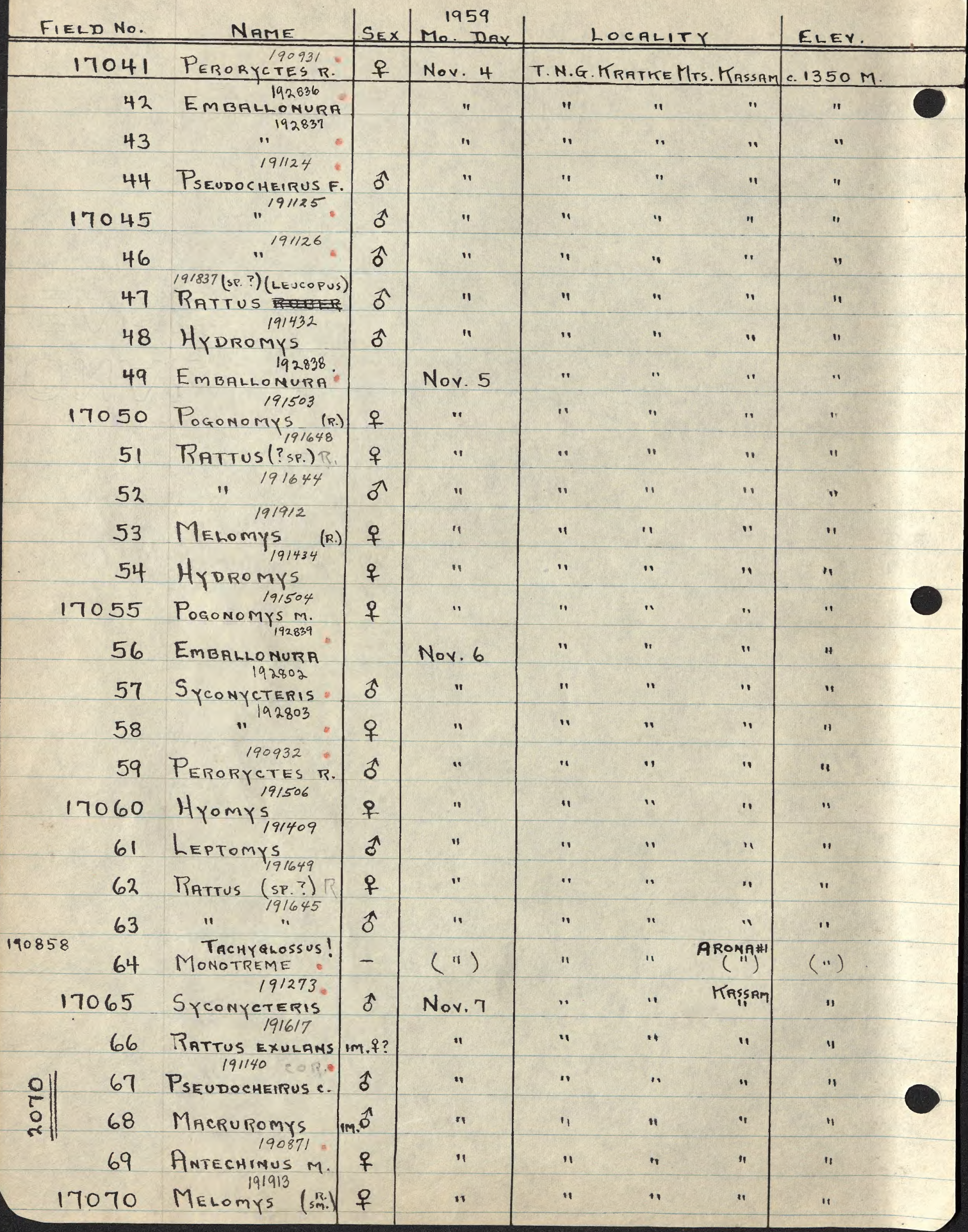




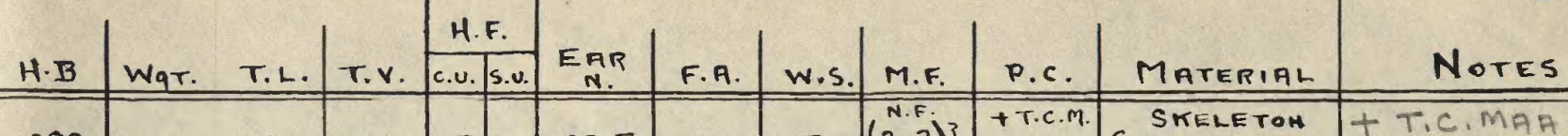

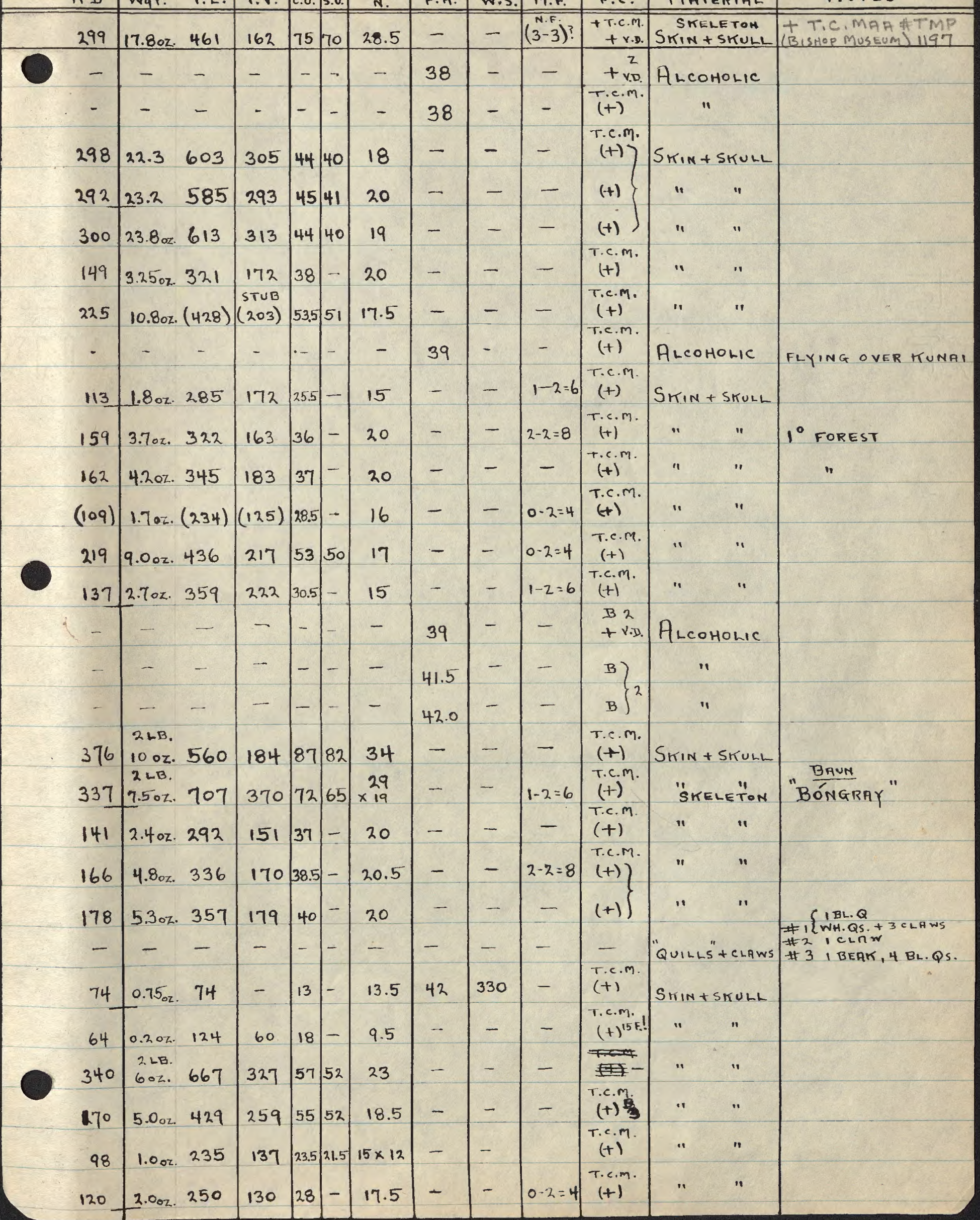



(70)

FIELD No

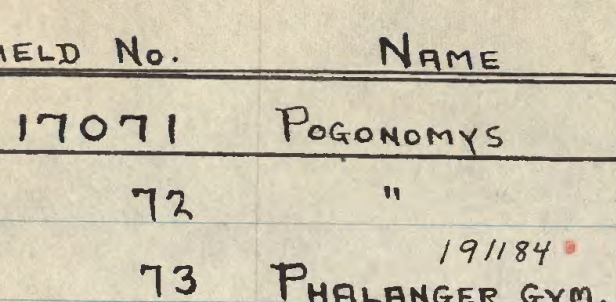
13 PHALANGER GYM. 74 PHILETOR 17075 P 191505 72 mys

192900

76 PHILETOR

77 Wa 192154

77 WALLABY 192164

78 Phalamger

79

17080 BandicoOT

192170

81

19217

82

192172

83 Satanellus

84 PhILetor

17085 PSEUdoCHEIRUS

86 liatrus ex.?

87 " $1916 / 8$

88 " 192400

89 MELOMYS

17090 HYDROMYs

91 MELOMY,

92 Rattus $R$.

93

191650

94

11 191652

191438

191438
0 mys 191293

96 NYCTIMENE

에 97 Ratrus $R$.

98

11 191654

99

+ 191655

17100 HYDROMYS SEX Mo. Tar

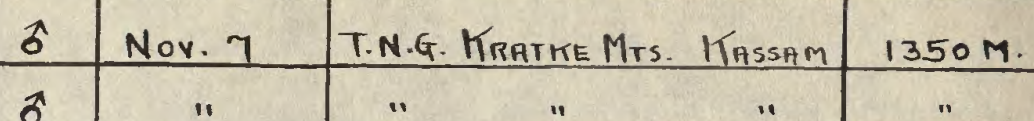
$\begin{array}{lll}6 & 11 \\ 0 & 11\end{array}$ ㅇ

Nor. 8 EMB ? im? $\hat{\sigma}$ ठิ

Nov. 9 $\sigma$
$q$
6 ( ") (11)

\begin{tabular}{|c|c|c|c|c|c|}
\hline$\delta$ & " & $"$ & $"$ & $"$ & \multirow{2}{*}{ c." } \\
\hline f & Nor. 8 & " & $"$ & $"$ & \\
\hline q. & $"$ & $"$ & $"$ & " & $"$ \\
\hline \multirow{7}{*}{$\begin{array}{c}\text { EMB. } \\
?\end{array}$} & " & $"$ & " & $"$ & $"$ \\
\hline & (") & $"$ & \multicolumn{2}{|c|}{$\begin{array}{l}\text { SRID TO BE FROM MARKF } \\
\text { HAM VRLLEY BELOW } \\
\text { KASSAM. }\end{array}$} & c. 1500 Fr.? \\
\hline & $(")$ & $"$ & \multicolumn{2}{|c|}{ KRATKE Mts. MuMár } & $\begin{array}{l}\text { ARONA } \\
\text { AY-2MI.E.OF }\end{array}$ \\
\hline & (") & $"$ & $"$ & $"$ & " \\
\hline & (ii) & $"$ & $"$ & $"$ & $"$ \\
\hline & (II) & $"$ & $"$ & " & " \\
\hline & (i) & " & $"$ &. & $"$ \\
\hline imp & $n$ & " & $"$ & KASSAM & $1350 \mathrm{M}$ \\
\hline ڤ & Nor. 9 & $"$ & " & 4 & " \\
\hline$\hat{\sigma}$ & " & $"$ & $"$ & $"$ & $"$ \\
\hline t & $"$ & $"$ & $"$ & " & " \\
\hline$q$ & $"$ & $"$ & $"$ & $"$ & $"$ \\
\hline$\hat{\sigma}$ & $" 1$ & $"$ & $"$ & " & "* \\
\hline & $\cdot$ & $"$ & $"$ & * & c. \\
\hline
\end{tabular}

\begin{tabular}{|c|c|c|c|c|c|}
\hline$\delta$ & " & $"$ & $"$ & $"$ & \multirow{2}{*}{ c." } \\
\hline f & Nor. 8 & " & $"$ & $"$ & \\
\hline q. & $"$ & $"$ & $"$ & " & $"$ \\
\hline \multirow{7}{*}{$\begin{array}{c}\text { EMB. } \\
?\end{array}$} & " & $"$ & " & $"$ & $"$ \\
\hline & (") & $"$ & \multicolumn{2}{|c|}{$\begin{array}{l}\text { SRID TO BE FROM MARKF } \\
\text { HAM VRLLEY BELOW } \\
\text { KASSAM. }\end{array}$} & c. 1500 Fr.? \\
\hline & $(")$ & $"$ & \multicolumn{2}{|c|}{ KRATKE Mts. MuMár } & $\begin{array}{l}\text { ARONA } \\
\text { AY-2MI.E.OF }\end{array}$ \\
\hline & (") & $"$ & $"$ & $"$ & " \\
\hline & (ii) & $"$ & $"$ & $"$ & $"$ \\
\hline & (II) & $"$ & $"$ & " & " \\
\hline & (i) & " & $"$ &. & $"$ \\
\hline imp & $n$ & " & $"$ & KASSAM & $1350 \mathrm{M}$ \\
\hline ڤ & Nor. 9 & $"$ & " & 4 & " \\
\hline$\hat{\sigma}$ & " & $"$ & $"$ & $"$ & $"$ \\
\hline t & $"$ & $"$ & $"$ & " & " \\
\hline$q$ & $"$ & $"$ & $"$ & $"$ & $"$ \\
\hline$\hat{\sigma}$ & $" 1$ & $"$ & $"$ & " & "* \\
\hline & $\cdot$ & $"$ & $"$ & * & c. \\
\hline
\end{tabular}
1 LOCALITY ELEY. o 우 o q

Nov, 10

UPPER Markitam; Watertice $450 \mathrm{M}$ f i1 우 ठิ Nor 11 "I 오 우 오 앙 


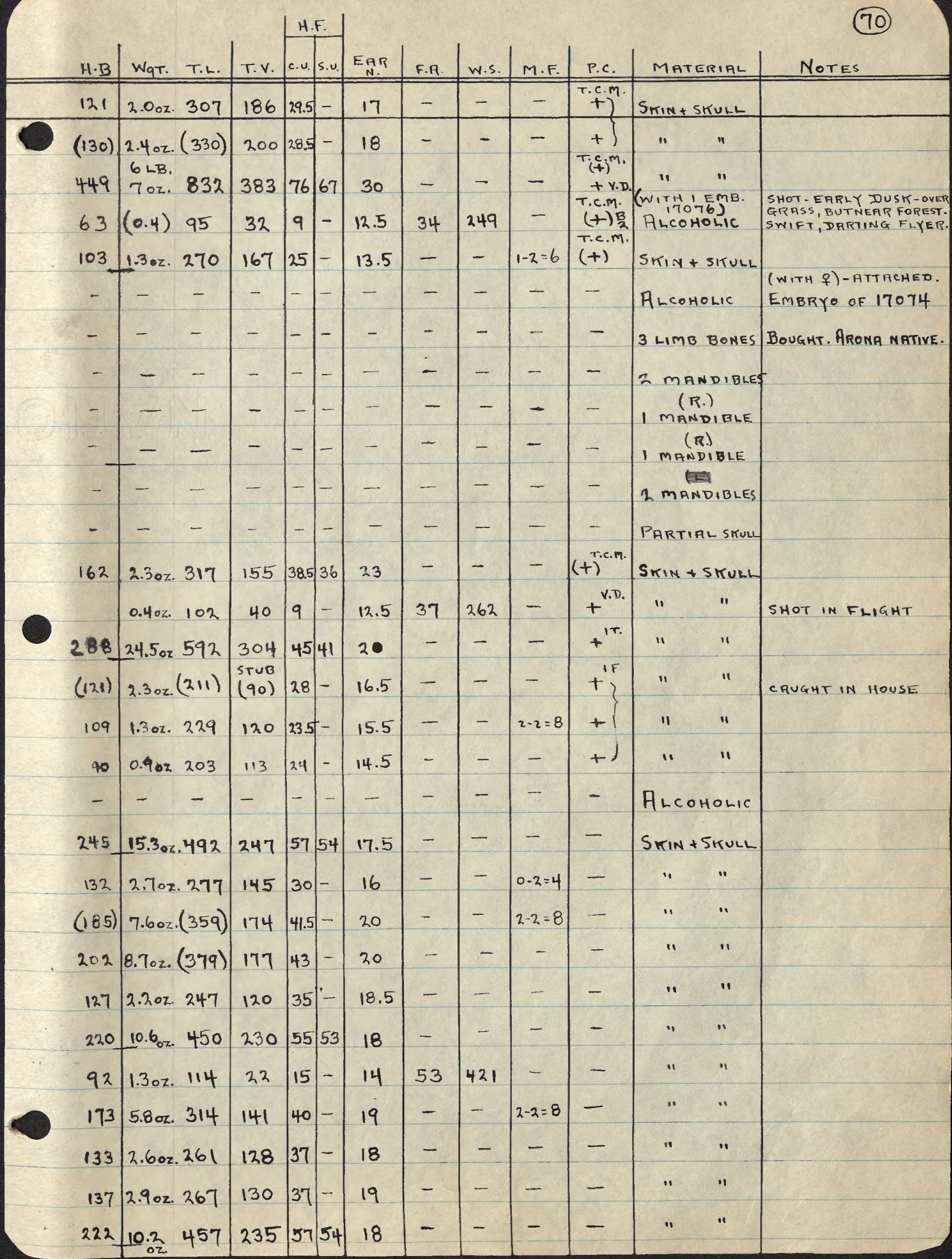




(71)

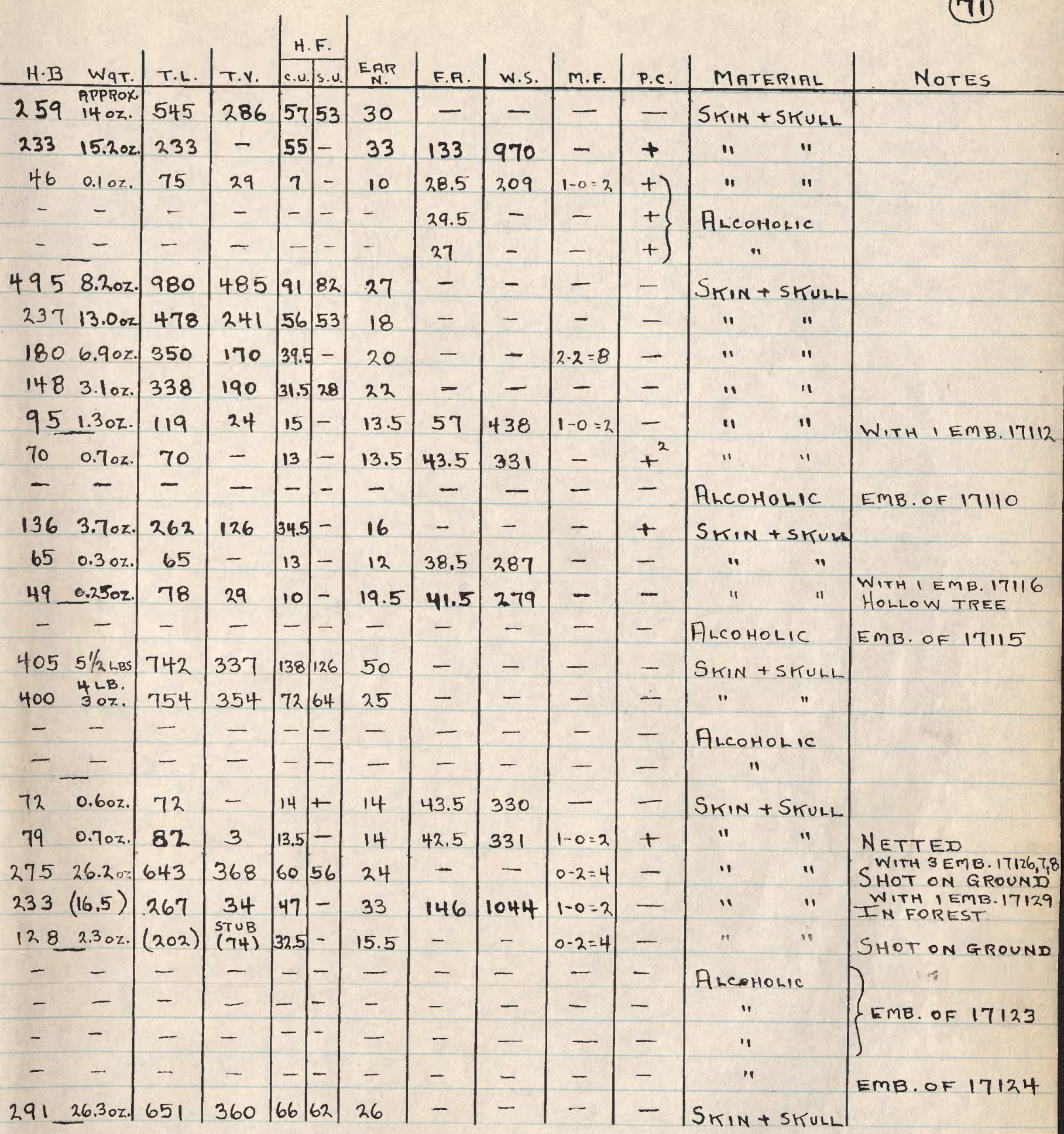



○

○

O 


\begin{tabular}{|c|c|c|c|c|c|c|c|}
\hline $\begin{array}{c}\text { FIELD } \\
\text { NO. }\end{array}$ & NAME & SEX & $\begin{array}{l}1959 \\
\text { Mo. DaY }\end{array}$ & \multicolumn{3}{|c|}{ LOCALITY } & ELEV. \\
\hline 17131 & $\begin{array}{l}\text { NYCTOPHILUS } \\
\text { (INSECTIVORUS BAT) }\end{array}$ & 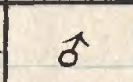 & Nov. 18 & \multicolumn{3}{|c|}{ T.N.G. MarkhamT.Valley; Umi R. } & $475 \mathrm{M}$ \\
\hline 32 & MELOMYS & $\hat{\sigma}$ & " & 1 & " & " & " \\
\hline 33 & 11 & im. है? & " & $" 1$ & $"$ & $"$ & " \\
\hline 34 & $\begin{array}{l}191170 \\
\text { DORCOPSIS. }\end{array}$ & $\pi$ & $"$ & $" 1$ & $"$ & " & MIN. $500 \mathrm{M}$. \\
\hline 17135 & $11^{191168}$. & 우 & $"$ & $"$ & $"$ & $"$ & 11 \\
\hline 36 & 11191171. & 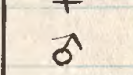 & $"$ & $" 1$ & $"$ & " & $"$ \\
\hline 37 & $\begin{array}{l}191 / 86 . \\
\text { PhALANGER GYM. }\end{array}$ & 우 & $"$ & $"$ & " & " & $"$ \\
\hline 38 & Phalamger OT. & 오 & "1 & $"$ & " & $"$ & $"$ \\
\hline 39 & Peroryctes R. & 오 & 11 & $"$ & $"$ & $"$ & $"$ \\
\hline 17140 & ECHYMIPERA & im. 9 & $"$ & $"$ & "1 & $"$ & $"$ \\
\hline 41 & 11190983 & im. 0 & $"$ & $"$ & $"$ & $"$ & $" 1$ \\
\hline 42 & MeLomys & 오 & 11 & $"$ & $"$ & $" 1$ & $475 \mathrm{~m}$. \\
\hline 43 & $11 / 9 / 874$ & $\hat{0}$ & " & 11 & " & " & 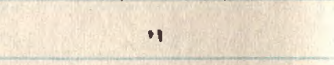 \\
\hline 44 & $\begin{array}{l}191295 \\
\text { NycTIMENE }\end{array}$ & $\pi$ & Nov. 19 & 11 & $"$ & " & $"$ \\
\hline 17145 & HuPROSIDEROS D. & $\vec{b}$ & Nor. 20 & $"$ & $"$ & $"$ & " \\
\hline 46 & $\begin{array}{l}191296 \\
\text { NyCTIMENE }\end{array}$ & $\hat{\delta}$ & $" 1$ & $"$ & $"$ & $" 1$ & " \\
\hline 47 & DENDROLAGUS & ठे & $" 1$ & $"$ & $\begin{array}{r}G W \\
\text { :MTS.NROF }\end{array}$ & $\lim ^{m}$ & $\geq 4100 \mathrm{~F}$ \\
\hline 48 & RHINOLOPHUS? & $q$ & Nov.21 & $"$ & ;MarkhamR & $U_{M I} R$. & $475 \mathrm{M}$ \\
\hline 49 & 4 to $192861 ?$ & & " & $"$ & " & $"$ & "1 \\
\hline 17150 & $\begin{array}{l}191297 \\
\text { NYCTIMENE. }\end{array}$ & $\widehat{\sigma}$ & $"$ & $\because$ & " & 11 & 11 \\
\hline 51 & RHINOLOPHUS & EMB. & $"$ & $" 1$ & $"$ & ' & $"$ \\
\hline 52 & DOBSONIA & 오 & Nor, 22 & $"$ & $"$ & $"$ & $"$ \\
\hline 53 & MELOMYS 19329 & & " & 11 & "' & " & " \\
\hline 54 & RHINOLOPHUS & & $"$ & " & " & " & $"$ \\
\hline 17155 & " 192863 & & $"$ & $"$ & $"$ & $" 1$ & " \\
\hline 56 & HIPPOSIDEROS & & Nov. 23 & $"$ & " & $"$ & \\
\hline 57 & 192866 & & $"$ & $"$ & $"$ & $"$ & \\
\hline 58 & "192867. & & " & $"$ & " & " & \\
\hline 59 & 11192868 & & " & 11 & $"$ & $" 1$ & \\
\hline 7160 & 11192869 & & $"$ & $"$ & $"$ & 11 & \\
\hline
\end{tabular}

17145 BREADTH ACROSS WING AT 5 TH FINGER - $99 \mathrm{~mm}$. SHOT JUST AT DUSK. FLYING SLOWLY THROUGH CLEARING NEAR BANK OF RIVER. $15-20$ FT HIGH. SLOW DIRECT FLIGHT. FIRST MISTAKEN FOR NIGHT JAR. 


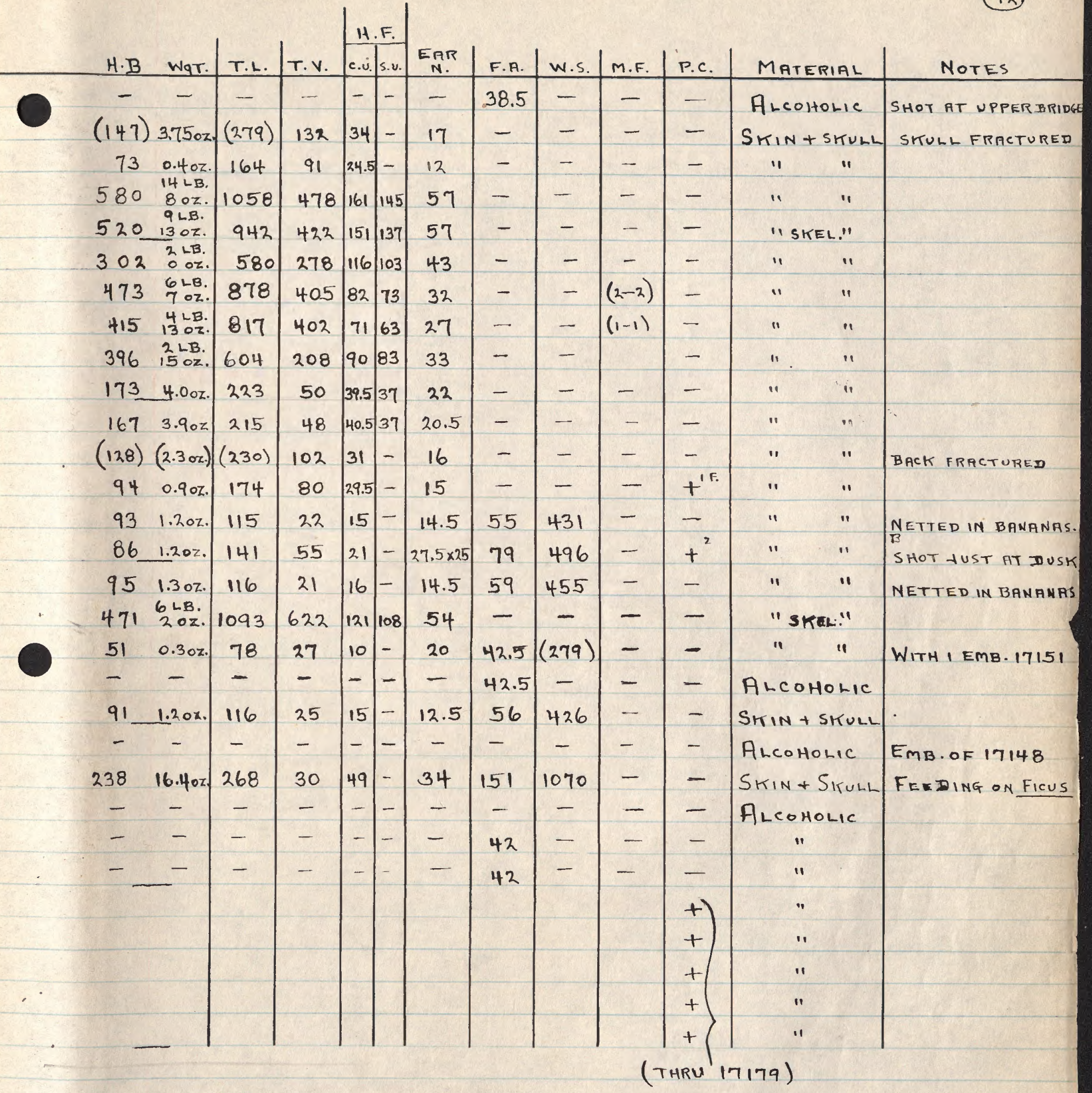

17147 Bought from Patrol Officer frank N. Harris, stationed at MAIPIT, bY JohN I. COLLINS. Harris bOUGHT THIS ANIMAL FROM NATIVESAT GWAISIRAM, AND BROUGHT IT ALIVE TO PATROL POST AT KAIPIT. 

At conCLUSION of 1959 ArChBOLd EXP.

To New guinea Van DeUsen went on ShORT

(1)

Collecting trip With B.J. Mar how (curator of mammals at the Australian Museum)

* to the upper allyn, N.S.W.

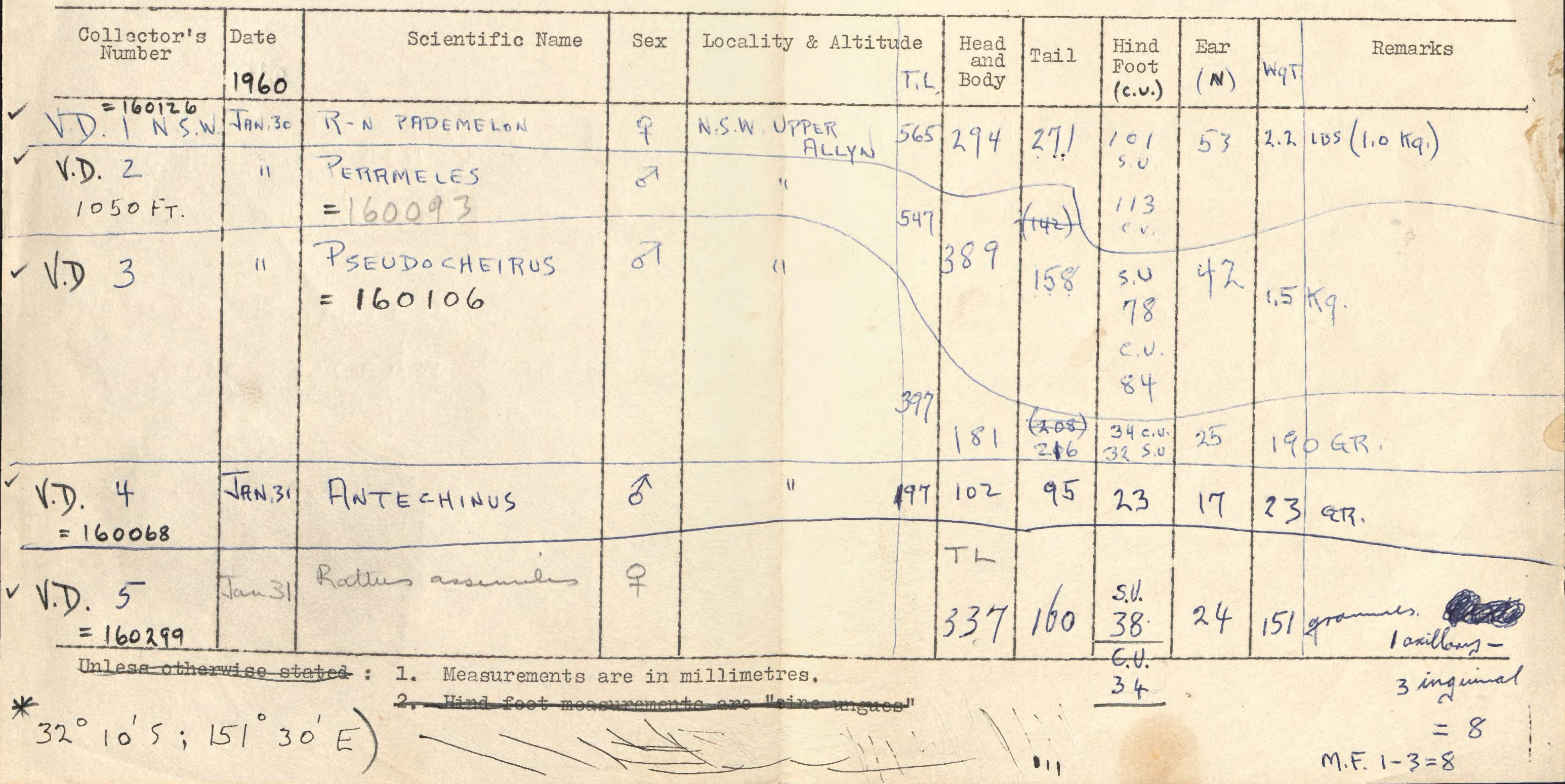



AUSTRALIAN MUSEUI

REGISTER OF SPECINENS COLTEOTED

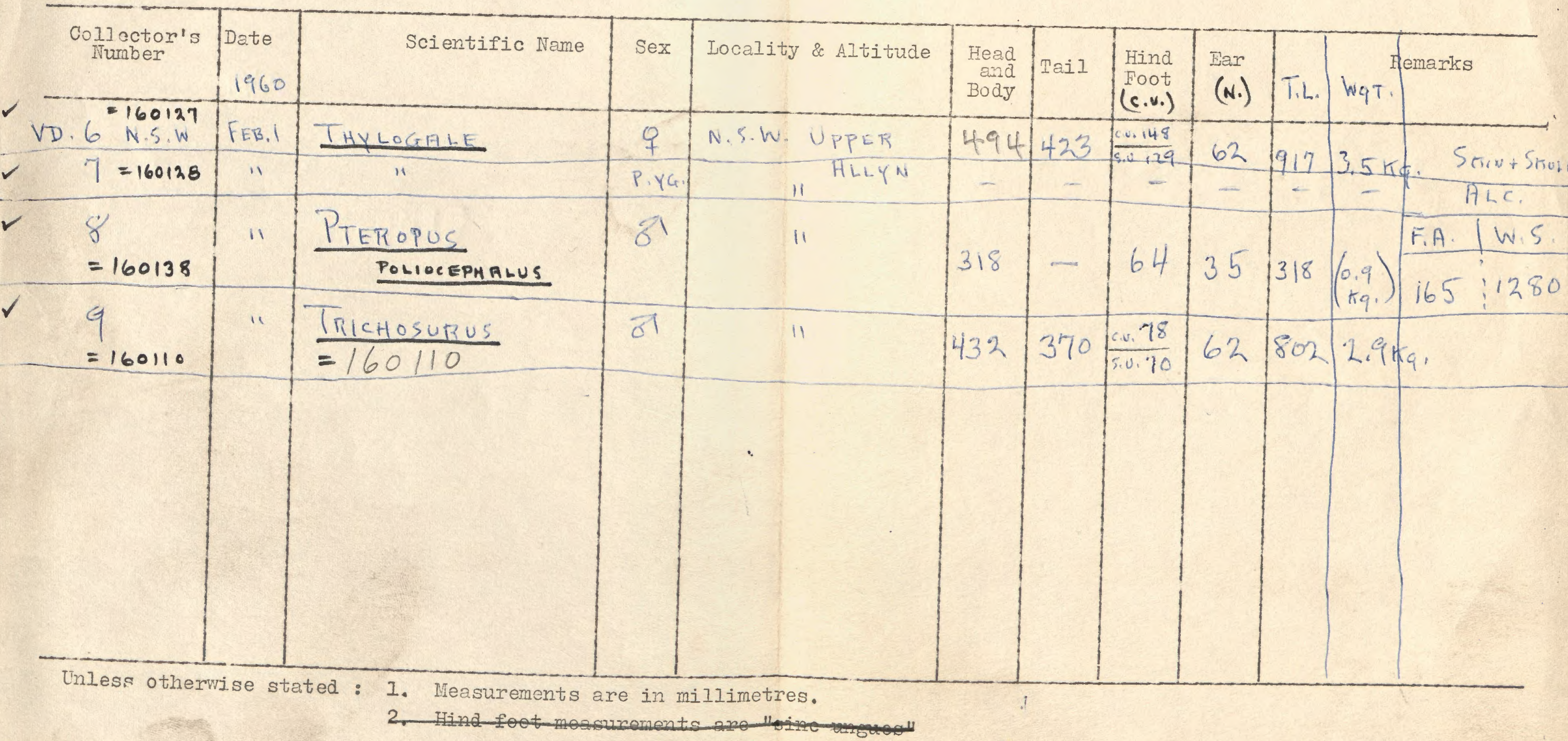




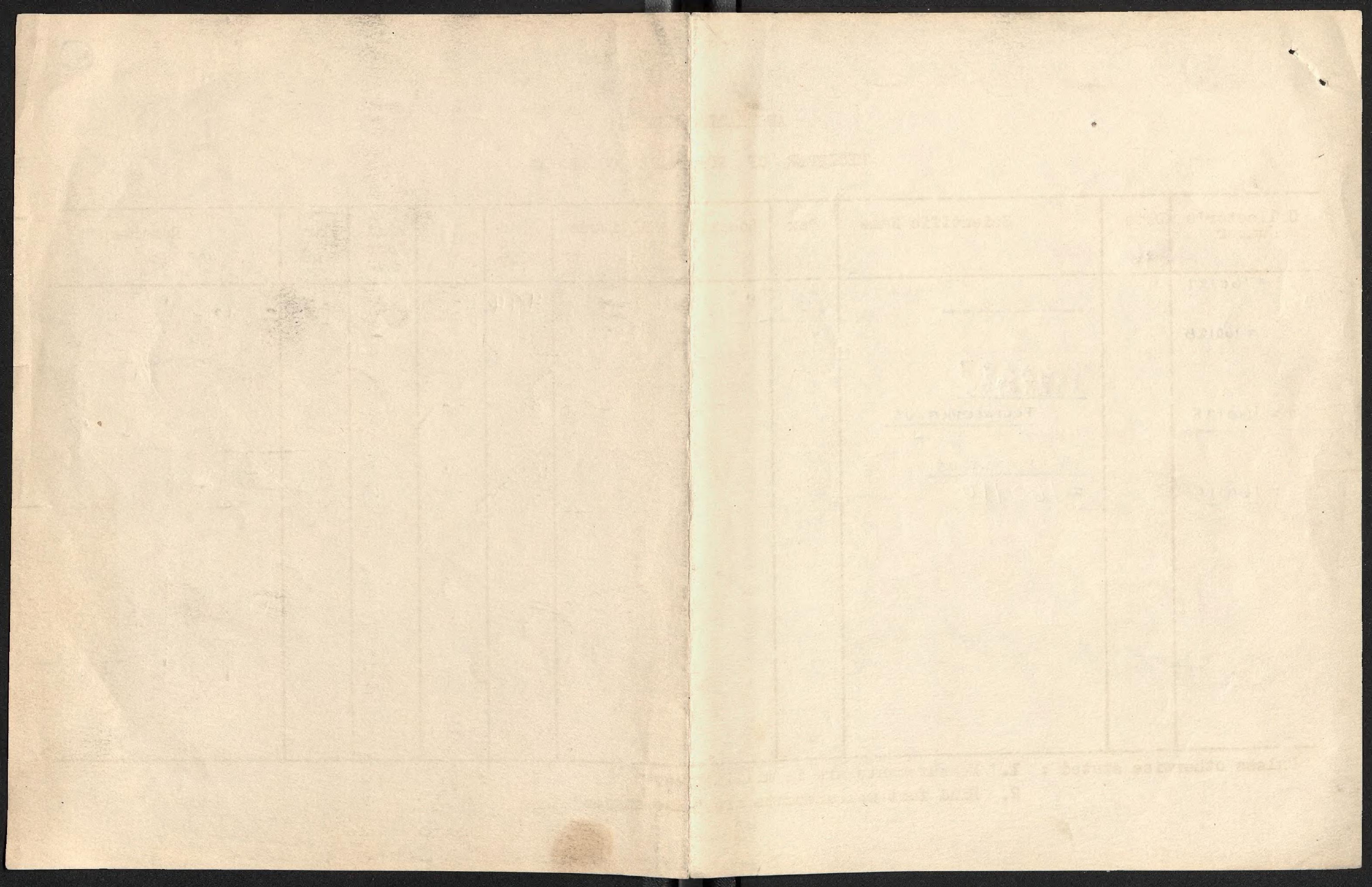



(73)

FIELD No:

\begin{tabular}{l|c}
1959 \\
SEX & $M_{0}$. DnY \\
\hline
\end{tabular}

17161 HIPPOSIDEROS

62

63

192872

64

17165

66

67

68

69

17170

71

72

73

192873

11.192874

11

192875

1192876

11

11

192878

11.192879

11

192880

11

192881

192882

74

17175

76

192883

11 192884

1

NoY. 23 N.N.G.

LOCALITY... .. ELEV.

NOV. 23 UPPER MARKHAM VALLEY:

ABOUT 5 MI. ABOVE (N)

IUNCTION OF UMI AND

"UMIN-RIVERS.
$=$ OFIM "

11
$" 1$




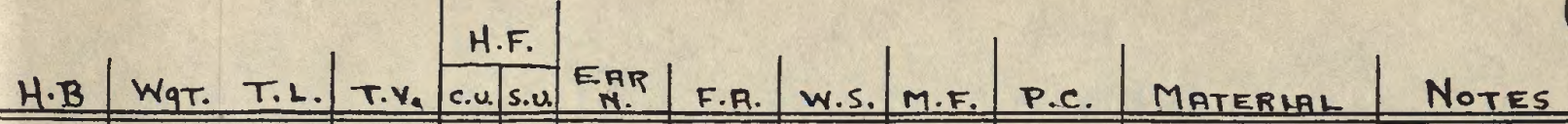

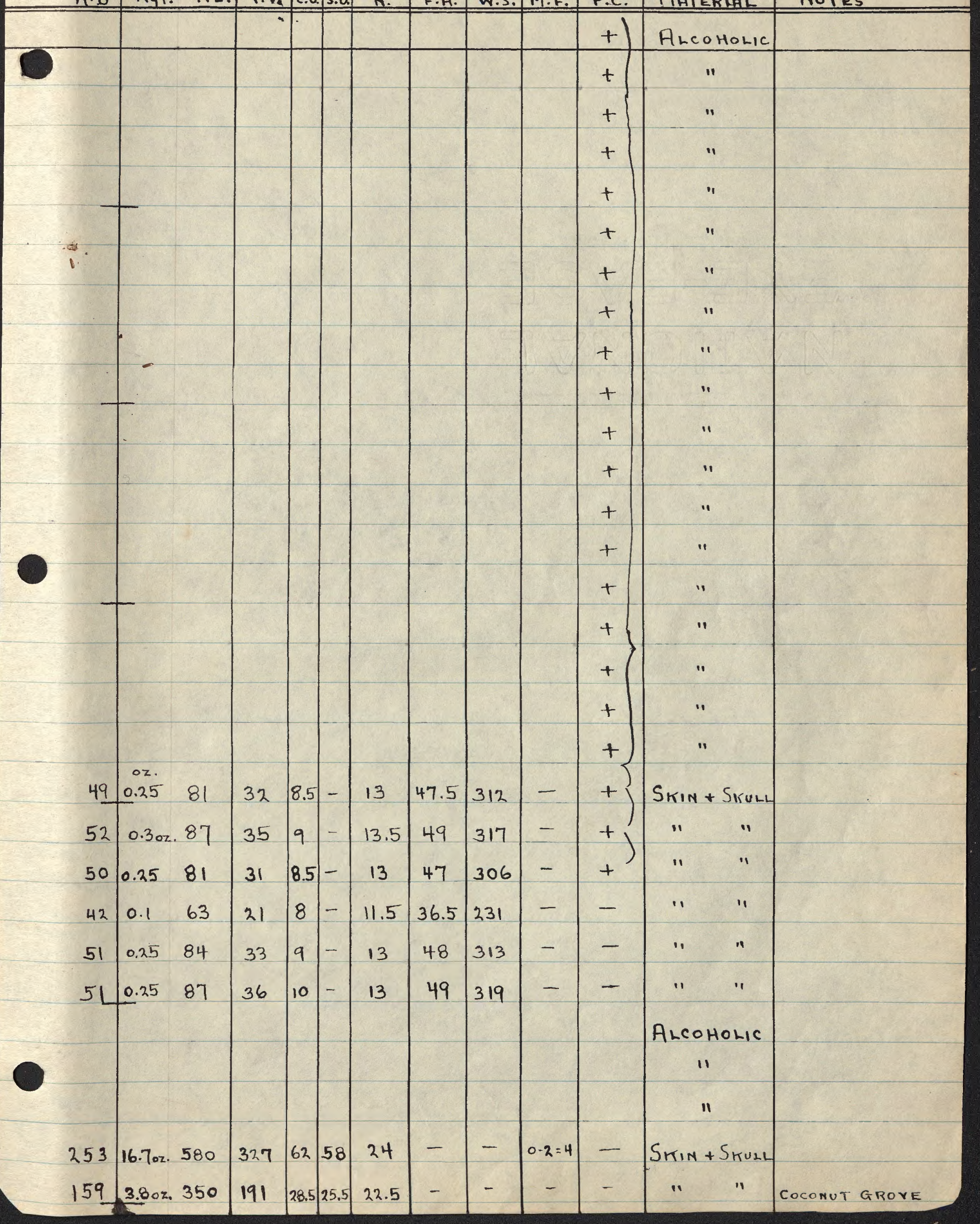




ELEV.

17191 MACROGLOSSUS 92 EMBALLONURA 192931+ 192932 emb. 93 Pipistrellus 94 EMBALLONURA 191282 17195 SYCONYCTERIS 96 Macroglossus 97 ECHYMIPERH R. 98 99

17200

193012 (s) 11 190986

\section{2} 193132 11 192933 * 93 Alent.

2 Pipistrellus of

3 EMBALLONURA 4 NYCTICEIUS?

17205 HYDROMYs 6 MELOMYS 7 Miniopterus (L) 8 NYCTIMENE 9 191299. 191283

17210 SYCONYCTERIS 11 191284

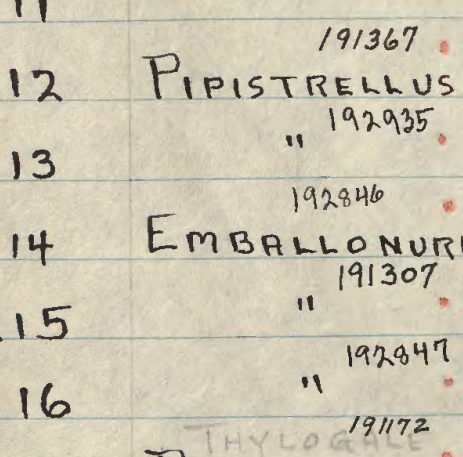

0
N.
N.

17 Dorcopsis 191187

18 Phalanger gym.

19 191185 191878 ㅇ

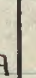

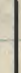
$\begin{array}{ll}11 & 193133 \\ 93012 & \text { (s) }\end{array}$ NoV. 23 T.N.G. MARKHAMY. UMIT. $480 \mathrm{M}$ $\hat{\sigma}$ Nov. 24 $q$ $\pi$ P. YG. P.YG \&

Nov. 25

".
오 ㅇ q $\hat{\sigma}$

Nov. 26 Nov. 27 $\hat{\sigma}$
ส $\delta$ б ๙ q (n) $\hat{\sigma}$ a
웅
a
P.YG 17220 MeLOMYS

\begin{tabular}{|c|c|c|c|}
\hline " & " & " & $" 1$ \\
\hline$"$ & " & $"$ & " \\
\hline " & $"$ & "1 & $" 1$ \\
\hline "1 & " & 11 & 11 \\
\hline$"$ & $" 1$ & $"$ & $\because$ \\
\hline " & 11 & " & c. $550 \mathrm{M}$ \\
\hline 11 & 11 & $"$ & 11 \\
\hline 11 & $" 1$ & " & 11 \\
\hline$\cdots$ & "1 & " & 11 \\
\hline 11 & " & 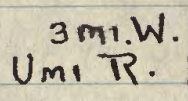 & c. $450 \mathrm{M}$ \\
\hline " & $"$ & $" 1$ & $"$ \\
\hline "1 & $"$ & 11 & " \\
\hline " & $"$ & 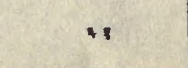 & $\because$ \\
\hline
\end{tabular}

" iUmi R. $475 \mathrm{M}$.

\begin{tabular}{|c|c|c|}
\hline .1 & " & $"$ \\
\hline$"$ & " & 11 \\
\hline$" 1$ & $" 1$ & $"$ \\
\hline$"$ & $"$ & $"$ \\
\hline " & " & $"$ \\
\hline$"$ & 11 & 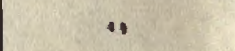 \\
\hline " & $3 \mathrm{m!} \cdot \mathrm{W}$ & $=450 \mathrm{M}$ \\
\hline " & 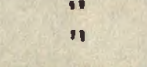 & $"$ \\
\hline$" 1$ & $\ddot{~}$ & $"$ \\
\hline 11 & $\ddot{~ " ~}$ & " \\
\hline$" 1$ & $\ddot{~}$ & " \\
\hline "1 & " & c. $550 \mathrm{M}$ \\
\hline$" 1$ & " & 1 \\
\hline$"$ & " & $"$ \\
\hline ". & mi $R$. & $475 \mathrm{M}$. \\
\hline
\end{tabular}




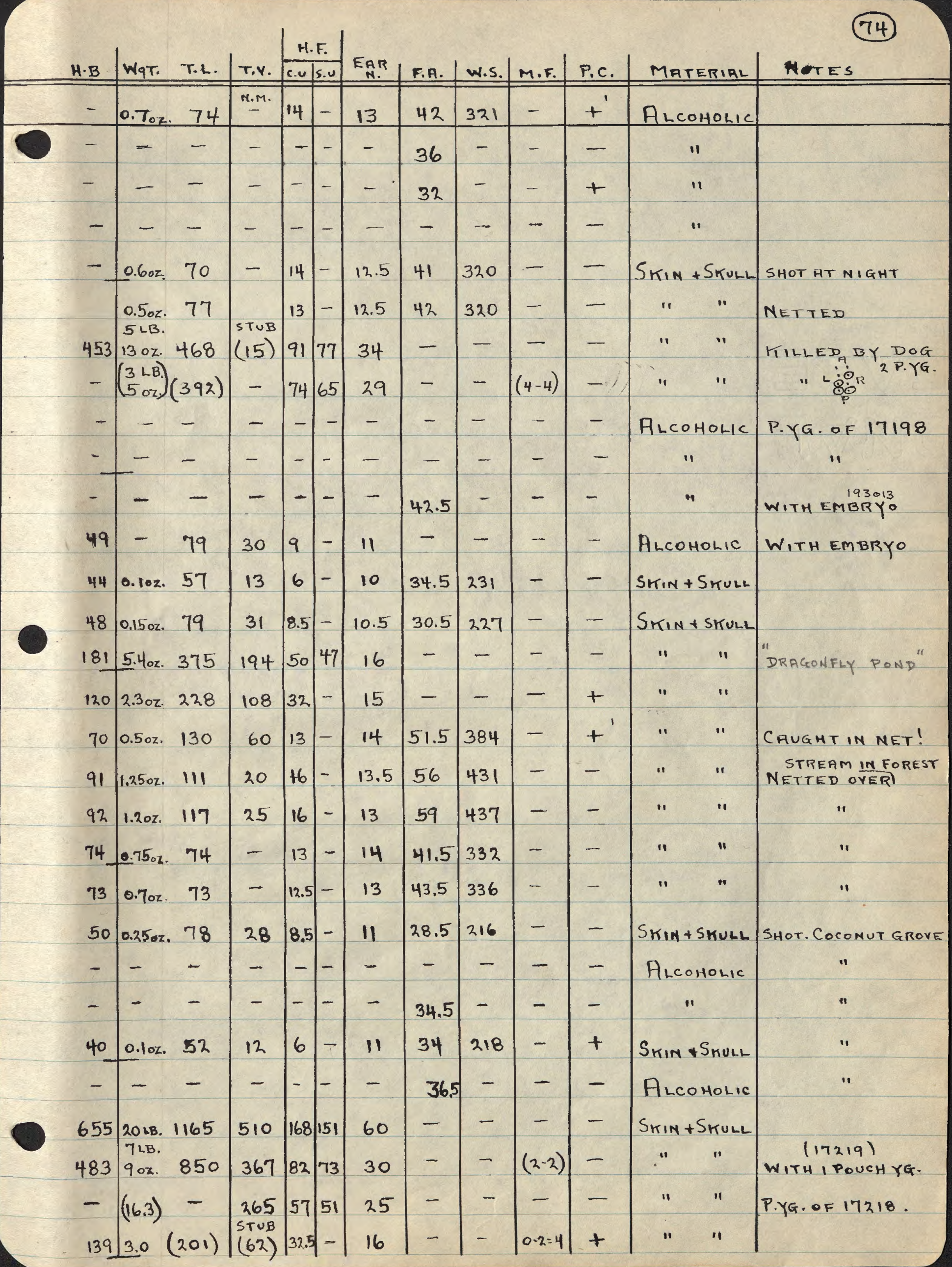



(75)

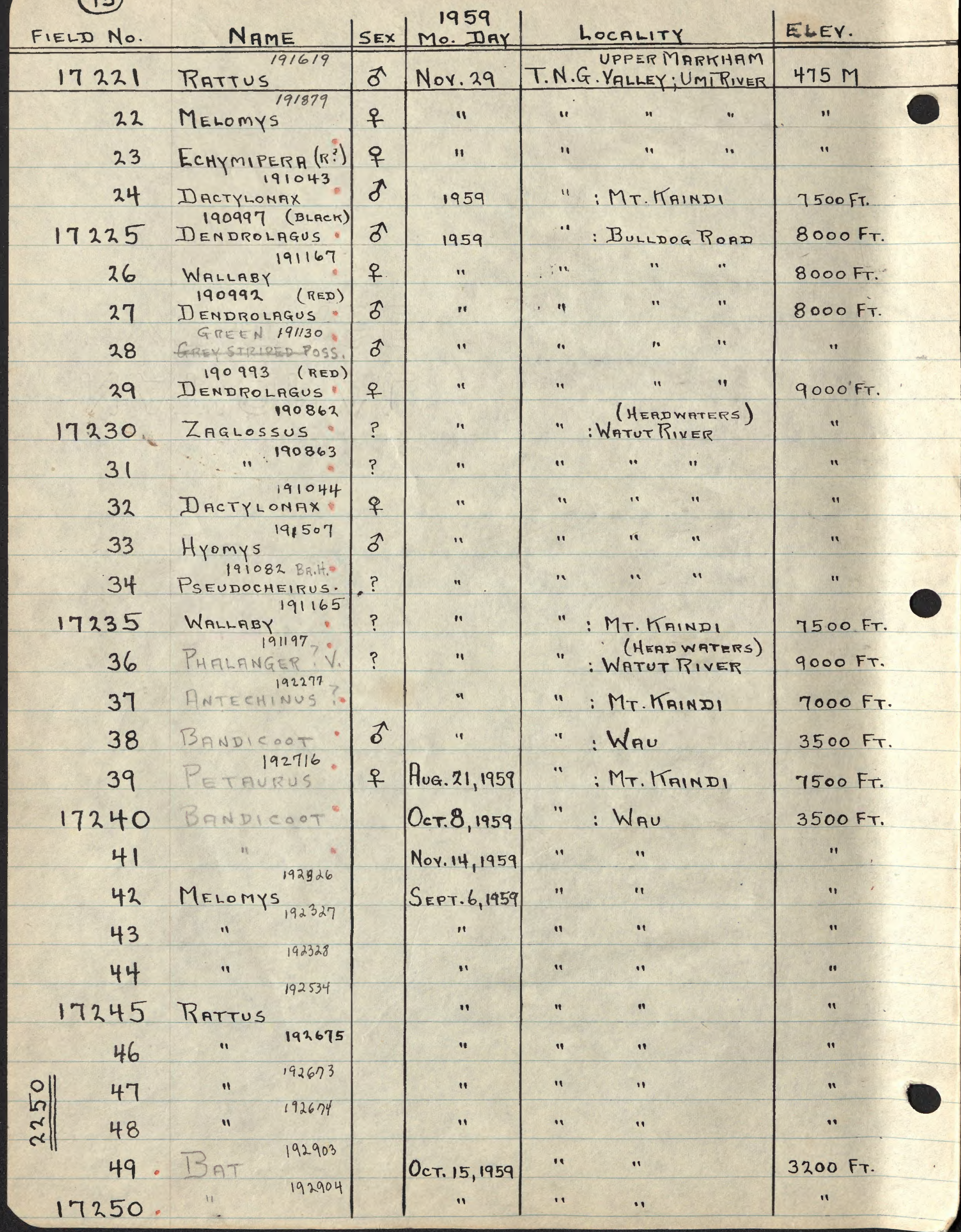




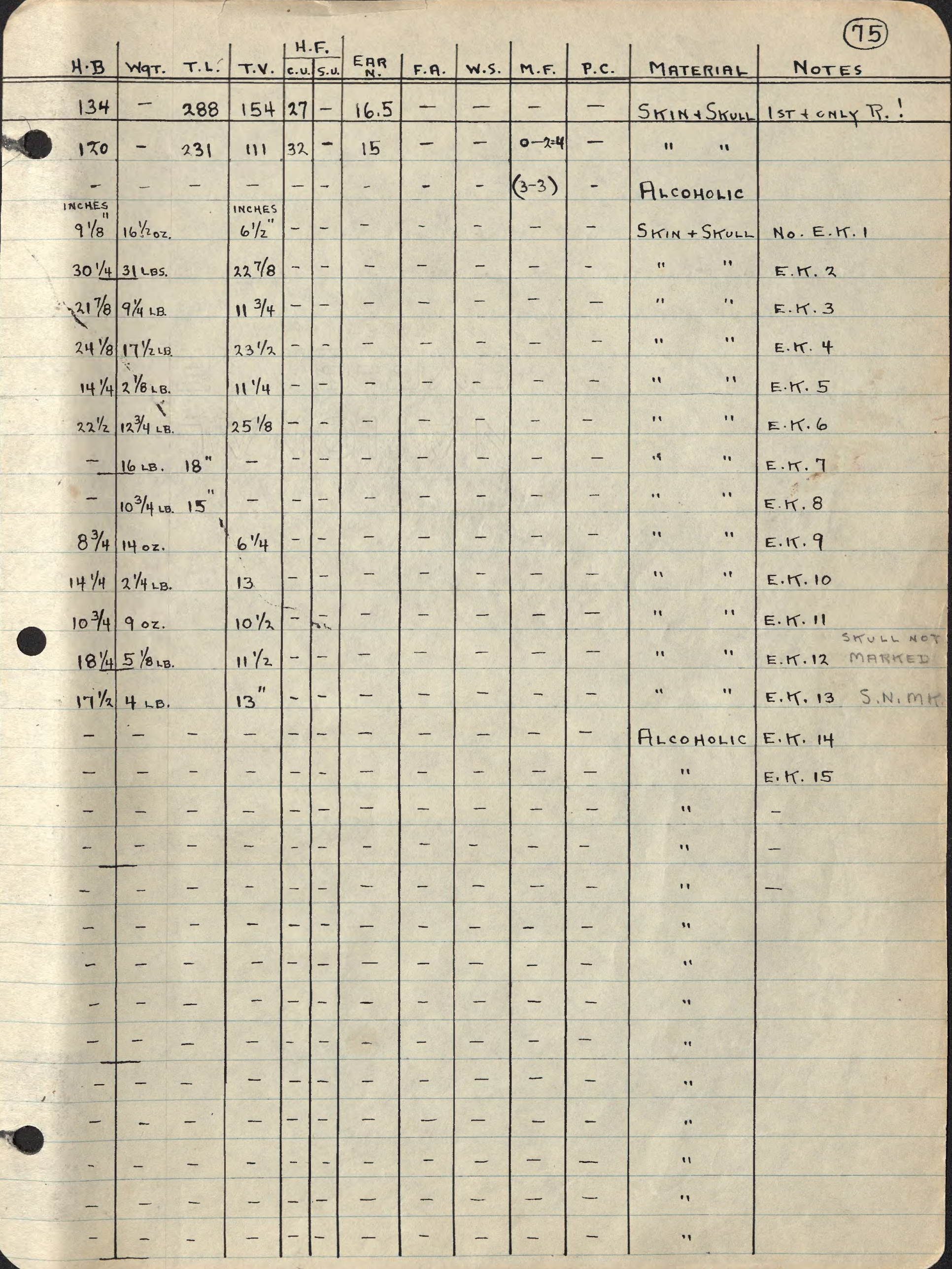




(76)

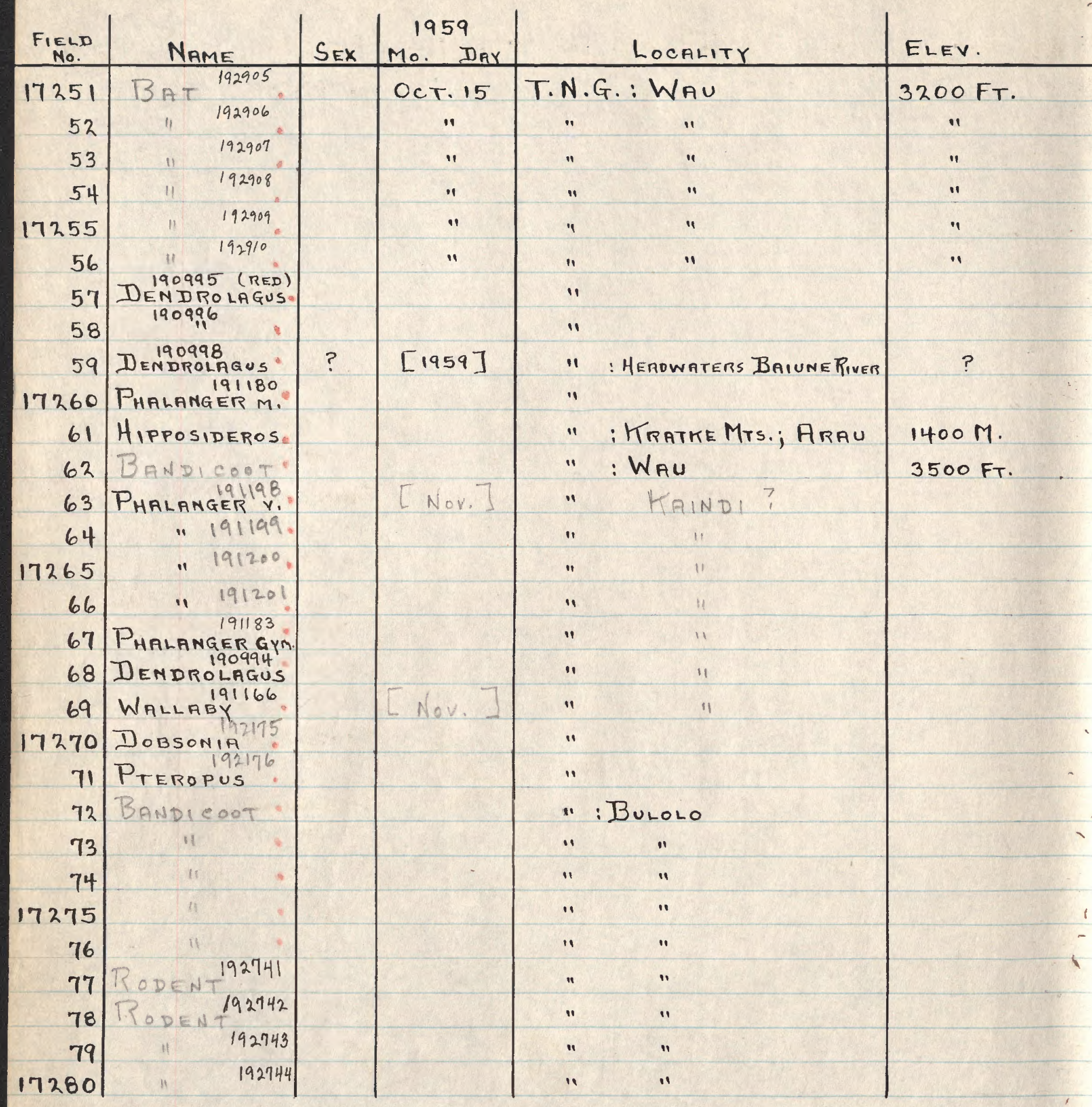

$17224-256$ GIFT FROM: HORACE CLISSOLD, WAU, T.N.G. COLLECTED BY BOYS WORKING AT THE GOLD MINE ON MT. MaINDI BELONGING TO H.C.'S FATHER-IN-LAW. 
(76)

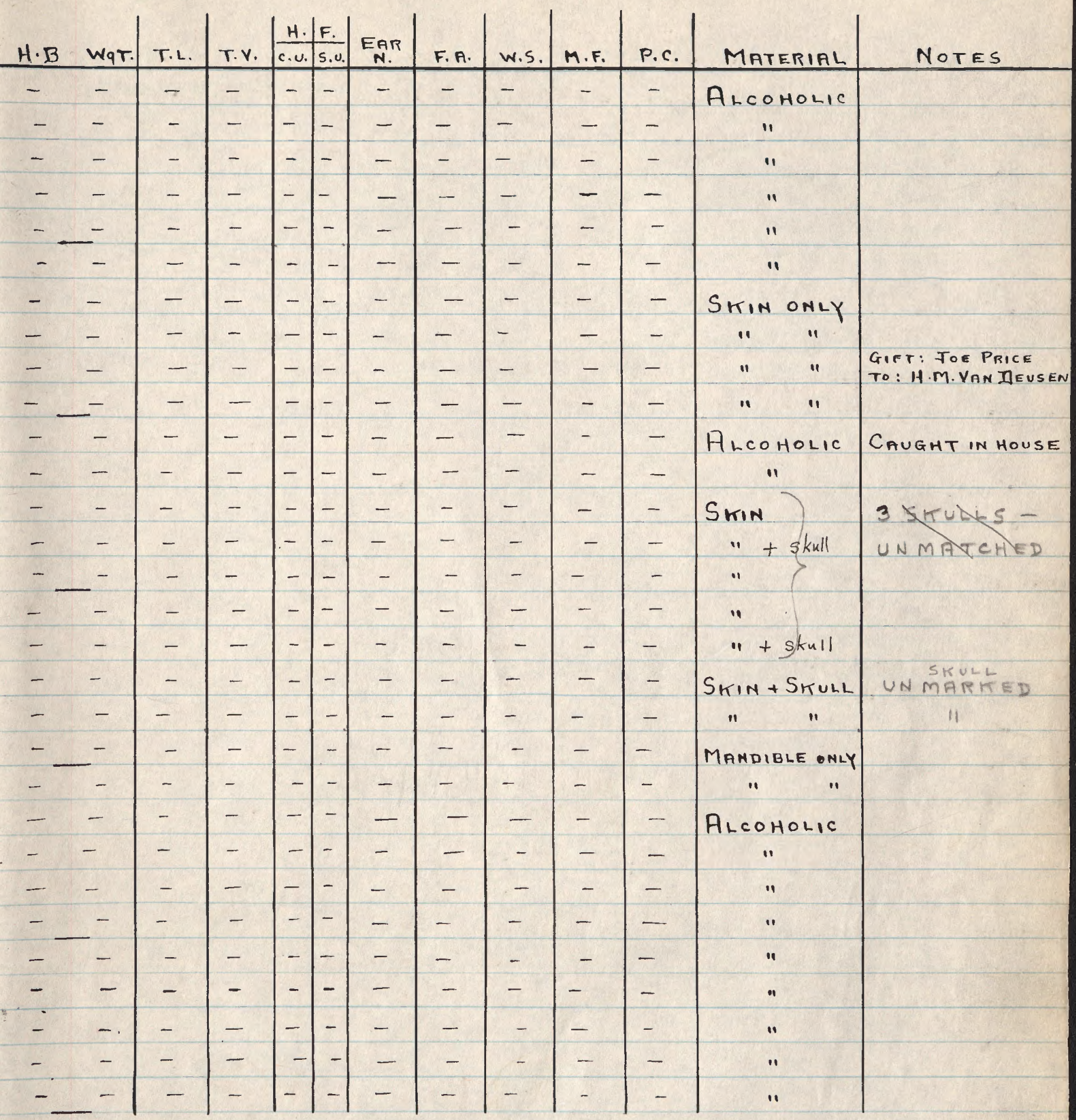

$17257,58,59$ GIFT: ToE PRICE* 172.60 K. CoLGINS-RUDEIE*
* GOHD MINERS 17261 Gift from: Mrs. WrLter Larner, Arau, T.n.g.

17270,71 Gift from: Mr. Keith Colhins-TRubie, Watut, T.N.G. 17272 -93 Gift from: Alam White, Forestry, Bulolo, T.n.g. 


(77)

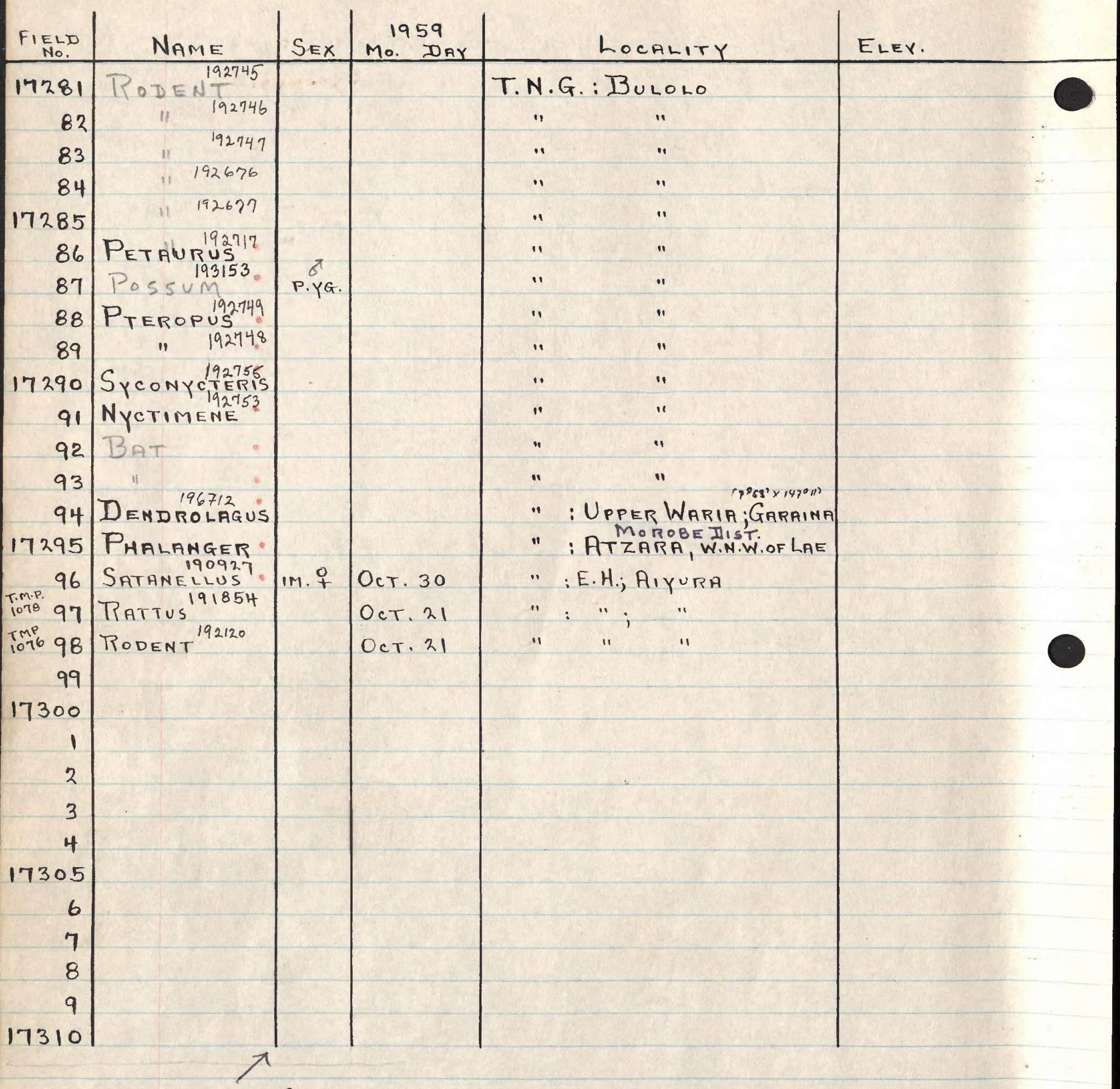

17294 GIFT fROM: DEPT. OF FORESTS, LAE, T.N.G. $17295 \rightarrow$ FOOTHILLS OF RAWLINSON MTS. ELEY.? UP TO $1800 \mathrm{FT}$.

2310 
(77)

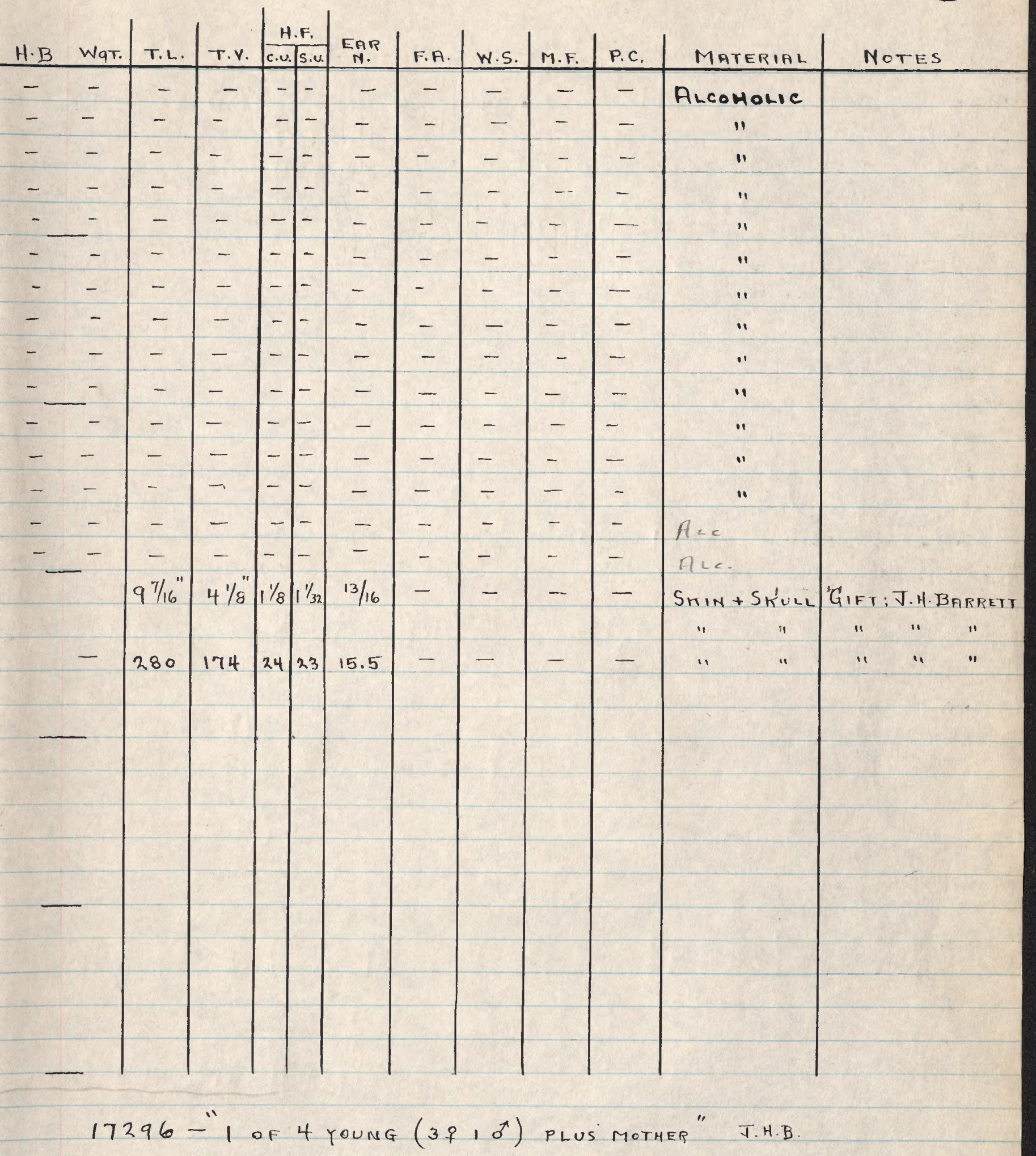

My Chimbu "BOY" = Tobram DEgOMBu 

(78)

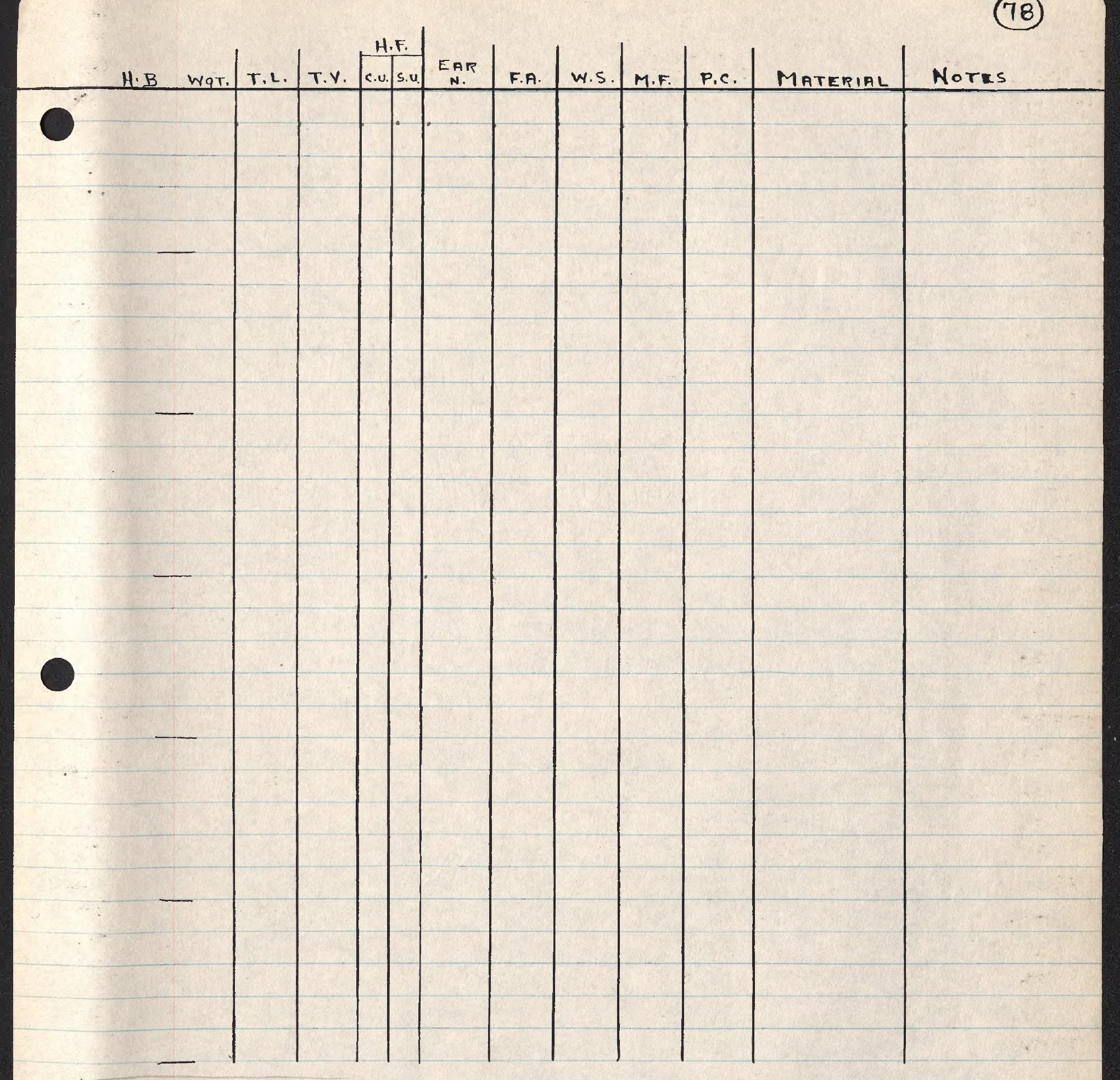





$$
\text { , }
$$ 





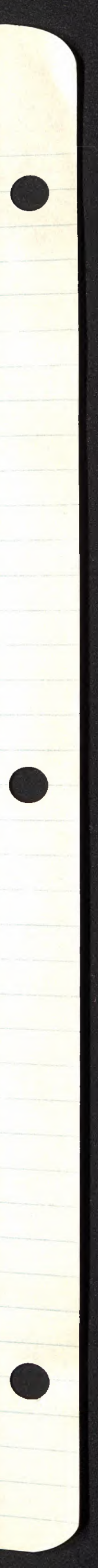


-

-

- 


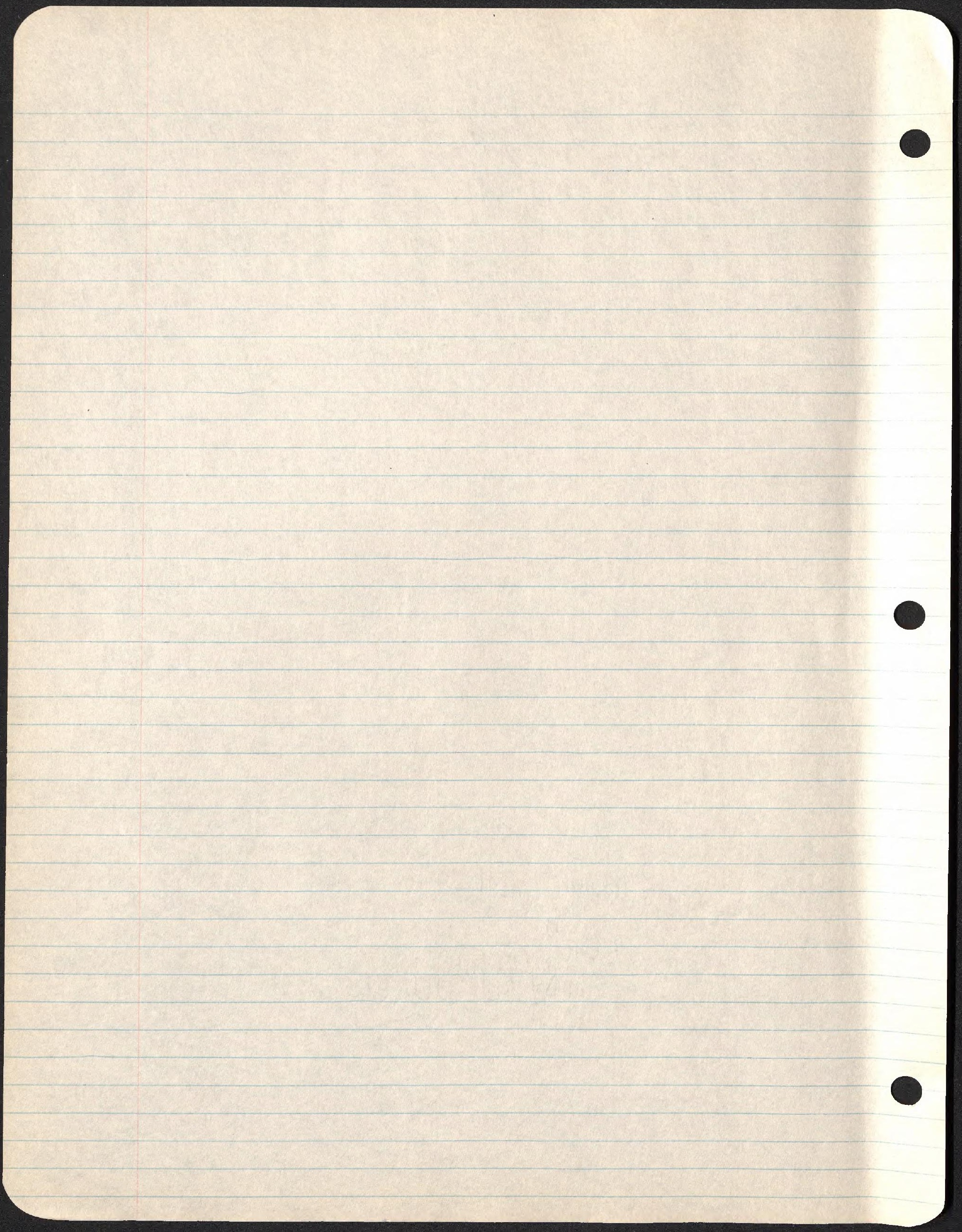





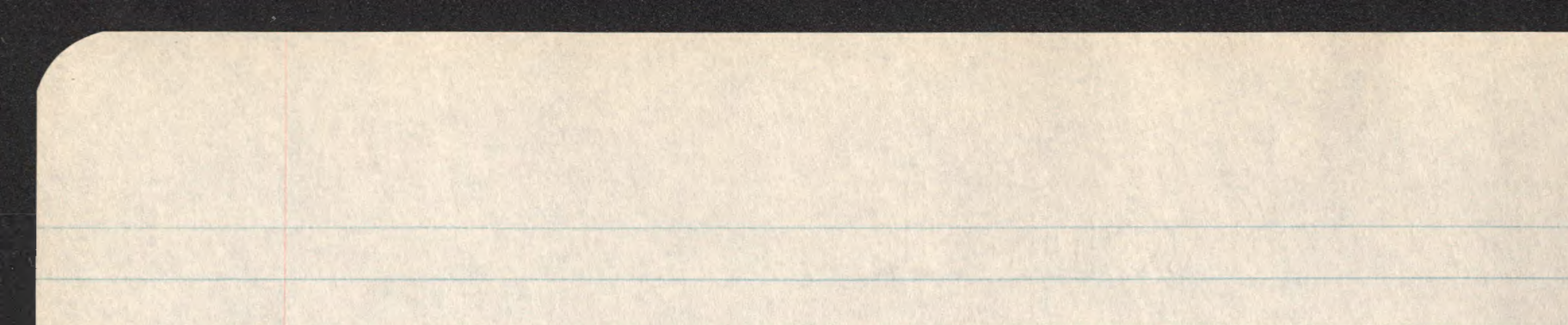






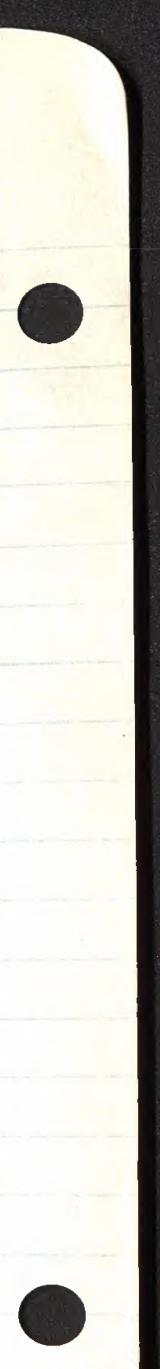










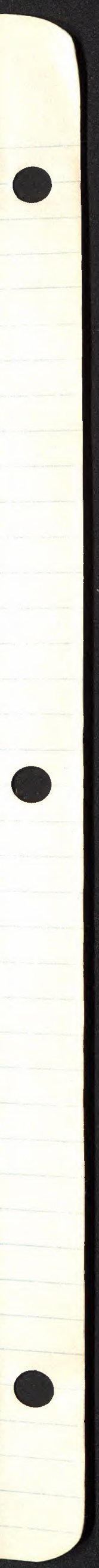




No.

SLIDES

LIST OF BLOOD SMEARS

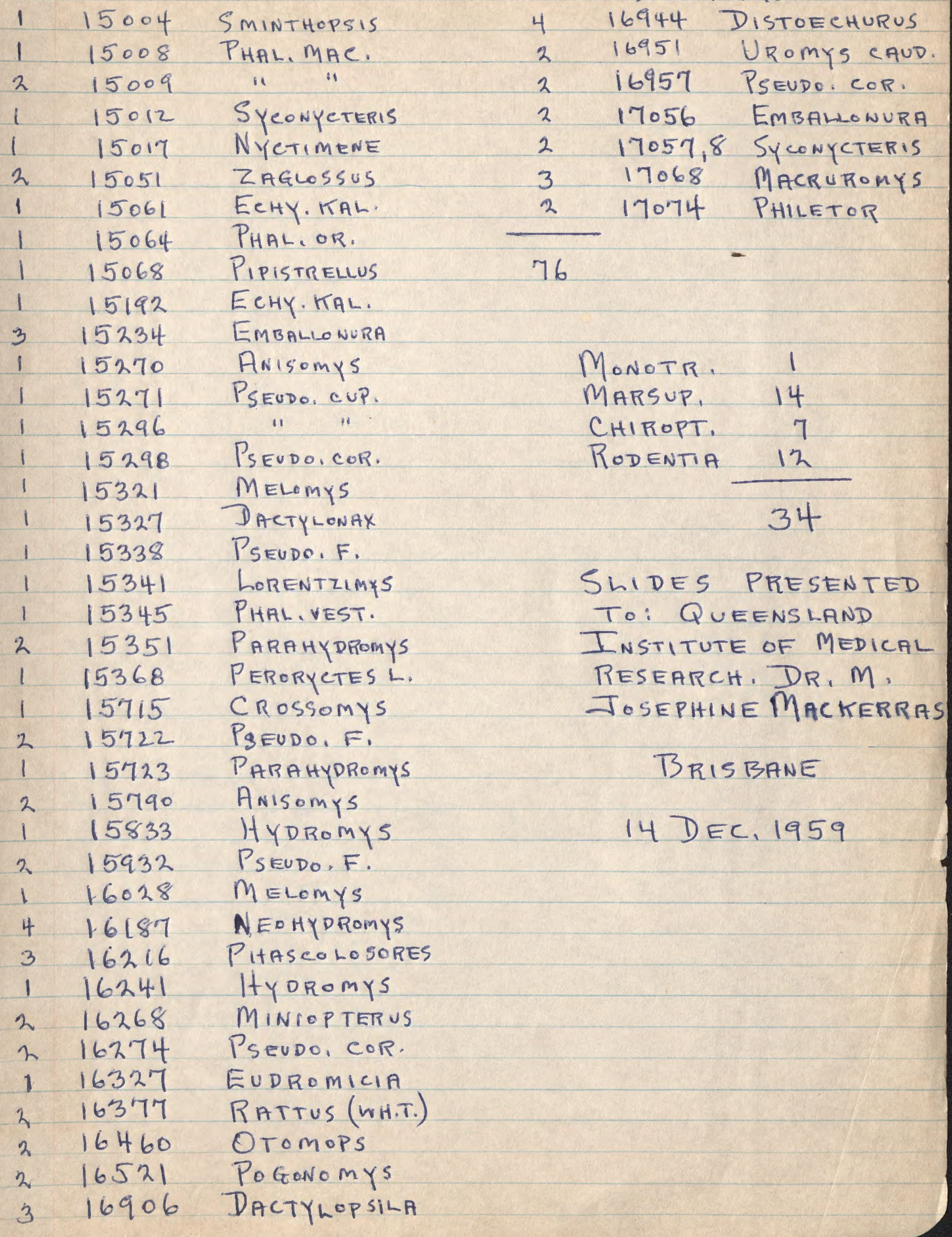






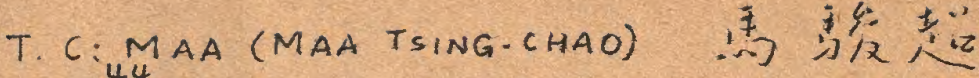

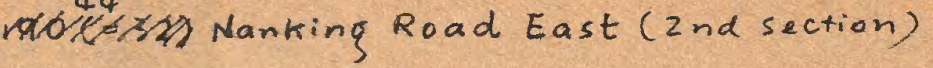

Taipei, Taiwan (Formosa)

or

Clo Bishop Museum

Honolulu 17 , Hawai: 

